\title{
PRECISE MEASUREMENT OF NEUTRINO AND ANTI-NEUTRINO DIFFERENTIAL CROSS SECTIONS ON IRON
}

\author{
by \\ Martin Mihaylov Tzanov \\ M.S., Sofia University, 1996 \\ M.S., University of Pittsburgh, 2003
}

Submitted to the Graduate Faculty of the School of Arts and Sciences in partial fulfillment of the requirements for the degree of Doctor of Philosophy

University of Pittsburgh 2005 


\section{UNIVERSITY OF PITTSBURGH}

\section{FACULTY OF ARTS AND SCIENCES}

This dissertation was presented

by

Martin Mihaylov Tzanov

It was defended on

November 14th, 2005

and approved by

Donna Naples, Associate Professor, Dept. of Physics and Astronomy

Anthony Duncan, Professor, Dept. of Physics and Astronomy

James Mueller, Associate Professor, Dept. of Physics and Astronomy

Vittorio Paolone, Associate Professor, Dept. of Physics and Astronomy

George Sparling, Professor, Dept. of Mathematics

Dissertation Director: Donna Naples, Associate Professor, Dept. of Physics and Astronomy 


\title{
ABSTRACT \\ PRECISE MEASUREMENT OF NEUTRINO AND ANTI-NEUTRINO DIFFERENTIAL CROSS SECTIONS ON IRON
}

\author{
Martin Mihaylov Tzanov, PhD \\ University of Pittsburgh, 2005
}

This thesis will present a precise measurement of the differential cross section for charged current neutrino and anti-neutrino scattering from iron. The $\mathrm{NuTeV}$ experiment took data during 1996-97 and collected $8.6 \times 10^{5} \nu$ and $2.4 \times 10^{5} \bar{\nu}$ charged-current (CC) interactions. The experiment combines sign-selected neutrino and antineutrino beams and the upgraded CCFR iron-scintillator neutrino detector. A precision continuous calibration beam was used to determine the muon and hadron energy scales to a precision of about a factor of two better than previous experiments. The structure functions $F_{2}\left(x, Q^{2}\right)$ and $x F_{3}\left(x, Q^{2}\right)$ are extracted and compared with theory and previous measurements. 


\section{TABLE OF CONTENTS}

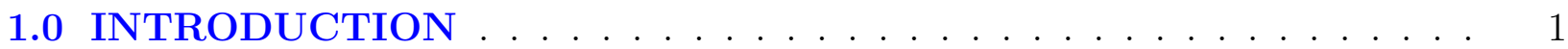

1.1 The Standard Model . . . . . . . . . . . . . . . . . . . . . . . . . . 2

1.2 Deep Inelastic Scattering _ . . . . . . . . . . . . . . . . . . 5

1.2.1 Kinematics of DIS . . . . . . . . . . . . . . . . 6

1.2.2 Neutrino-Nucleon Charge Current Differential Cross Section . . . . . . 7

1.3 Quark Parton Model . . . . . . . . . . . . . . . . . . . . . 10

1.4 Quantum Chromodynamics .................... 16

1.4 .1 Renormalization . . . . . . . . . . . . . . . 17

1.4 .2 Factorization . . . . . . . . . . . . . . . . . . . . . . 19

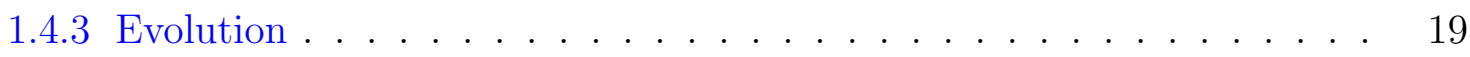

1.4.4 Non-Perturbative QCD Effects . . . . . . . . . . . . 21

1.4.4.1 Higher Twist Effects . . . . . . . . . . . . . . . 21

1.4.4.2 Target Mass Effects . . . . . . . . . . . . . . 21

2.0 DETECTOR AND BEAMLINE . . . . . . . . . . . . . . . 23

$2.1 \mathrm{NuTeV}$ Beamline . . . . . . . . . . . . . . . . . . . . . . . 23

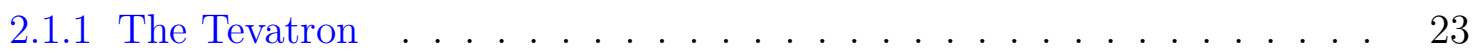

2.1.2 Neutrino Beamline . . . . . . . . . . . . . . . . . . 25

2.1 .3 Beam Timing Structure . . . . . . . . . . . . . . . 26

2.1 .4 Calibration Beam . . . . . . . . . . . . . . . 28

$2.2 \mathrm{NuTeV}$ Detector . . . . . . . . . . . . . . . . . . . . . . 29

2.2 .1 Target Calorimeter . . . . . . . . . . . . . . . . 30

2.2.1.1 Scintillator Counters . . . . . . . . . . . . . . . . 31 
2.2.1.2 Drift Chambers . . . . . . . . . . . . . . . 32

2.2 .2 Muon Spectrometer . . . . . . . . . . . . . . . . . 34

2.3 Readout Electronics and Event Triggering . . . . . . . . . . . . . . . . . 38

2.3.1 Drift Chamber Signal Read-out . . . . . . . . . . . . . . . . 38

2.3.2 PMT Signal Read-out . . . . . . . . . . . . . . . . . . 38

2.3 .3 Event Triggers . . . . . . . . . . . . . . . . . . . . . . . . . . 39

3.0 DETECTOR CALIBRATION AND DATA ANALYSIS . . . . . . . 43

3.1 Hadron Energy Reconstruction and Calibration . . . . . . . . . . . . . 43

3.2 Muon Angle Reconstruction . . . . . . . . . . . . . . . . . . . . . . 49

3.3 Muon Energy Reconstruction and Calibration . . . . . . . . . . . . . . . . 49

3.3.1 Muon Momentum in the Toroid . . . . . . . . . . . . . . . 50

3.3 .2 Total Muon Energy . . . . . . . . . . . . . . . . . . . . . . 50

3.3.3 Muon Energy Calibration . . . . . . . . . . . . . . . . . . 52

3.3.4 Muon Energy Resolution Function . . . . . . . . . . . . . . . . 54

3.4 Event Selection and Data Samples . . . . . . . . . . . . . . 56

3.4 .1 Quality and Fiducial Volume Cuts . . . . . . . . . . . . 56

3.4.2 Tracking and Geometric Cuts . . . . . . . . . . . . . 58

3.4 .3 Total Cross Sample . . . . . . . . . . . . . . . . . . . . . . 59

3.4 .4 Flux Sample . . . . . . . . . . . . . . . . . . . . . . . . 59

3.4.5 Structure Function Sample . . . . . . . . . . . . . . . . 59

4.0 MONTE CARLO EVENT SIMULATOR . . . . . . . . . . . . . 61

4.1 Cross Section Model . . . . . . . . . . . . . . . . . . . . . 62

4.1 .1 PDF Parameterization . . . . . . . . . . . . . 63

4.1.1.1 Valence Evolution . . . . . . . . . . . . . . . . . . . . 64

4.1.1.2 Light Sea Evolution . . . . . . . . . . . . . . . . 66

4.1 .1 .3 Strange Sea . . . . . . . . . . . . . . . 68

4.1.1.4 Modifications to the PDF parameterization . . . . . . . . . 69

4.1 .2 Longitudinal Structure Function . . . . . . . . . . . . . . . 71

4.1 .3 DIS Differential Cross Section . . . . . . . . . . . . . 71

4.1.3.1 Charm Production Cross Section . . . . . . . . . . 72 
4.1.3.2 Non-Isoscalar Target Cross Section . . . . . . . . . . . . . . 74

4.1.3.3 Propagator Term . . . . . . . . . . . . . . 75

4.1 .4 Radiative Corrections . . . . . . . . . . . . . . . . . . . . . . 75

4.1 .5 Cross Section Fit . . . . . . . . . . . . . . . 76

4.2 Event Generation . . . . . . . . . . . . . . . . . . . . . 80

4.3 Detector Model . . . . . . . . . . . . . . . . . . . . . . . . . 83

4.3.1 Muon Energy Smearing and Reconstruction . . . . . . . . . . . . . 83

4.3.1.1 Muon Energy Reconstruction . . . . . . . . . . . . . 84

4.3 .2 Muon Angle Smearing . . . . . . . . . . . . . . . . . . . . . 85

4.3.3 Hadron Energy Smearing and Reconstruction . . . . . . . . . . . . . 85

5.0 NEUTRINO FLUX AND NEUTRINO TOTAL CROSS SECTION . . 86

5.1 Flux Extraction Method . . . . . . . . . . . . . . 86

5.2 Flux Extraction . . . . . . . . . . . . . . . . . . . . . 89

5.2.1 Flux Systematic Uncertainties . . . . . . . . . . . . . . . 98

5.3 Total Neutrino Cross Section and Flux Normalization . . . . . . . . . . . . 103

5.4 Anti-neutrino to Neutrino Cross Section Ratio . . . . . . . . . . . . . . . . 106

6.0 DIFFERENTIAL CROSS SECTION . . . . . . . . . . . . . . . 111

6.1 Differential Cross Section Extraction . . . . . . . . . . . . . . 111

6.2 Iteration Procedure . . . . . . . . . . . . . . . . . . . . 118

6.3 Systematic Uncertainties . . . . . . . . . . . . . . . . . . . . . 124

6.4 Results and Comparison with CCFR and CDHSW . . . . . . . . . . 126

6.5 NuTeV-CCFR High x Discrepancy . . . . . . . . . . . . . . . . . . . 131

7.0 STRUCTURE FUNCTIONS . . . . . . . . . . . . . . . 135

7.1 Structure Function From One Parameter Fits . . . . . . . . . . . . . . 135

7.2 Structure Functions from Two Parameter Fit . . . . . . . . . . . . . . . . . 140

7.3 Comparison with Theory and QCD . . . . . . . . . . . . . 141

7.4 Logarithmic Slopes . . . . . . . . . . . . . . . . . . . . . 146

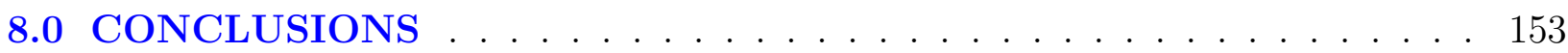

BIBLIOGRAPHY . . . . . . . . . . . . . . . . 155

APPENDIX A. THE NUTEV COLLABORATION . . . . . . . . . . . . 158 
APPENDIX B. PUBLICATION . . . . . . . . . . . . . . . . . . . 159 


\section{LIST OF TABLES}

1.1 Quarks are ordered in three generations. Electric charge is given as a fraction of the electric charge of the proton. . . . . . . . . . . . . . . 3

1.2 Leptons are ordered in three generations. Electric charge is given as a fraction of the electric charge of the proton. . . . . . . . . . . . . . . 4

1.3 Fundamental interactions and their relative strengths. . . . . . . . . . . . . 4

2.1 Length, radiation length and interaction length for the components of one

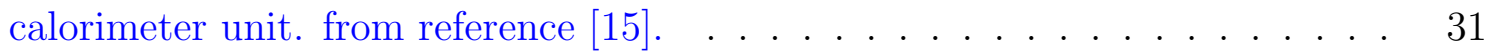

2.2 Number of neutrino gate events for different triggers recorded during $\mathrm{NuTeV}$

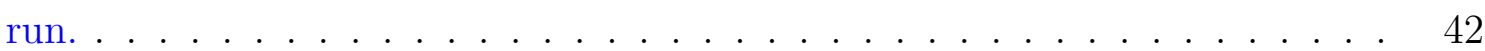

4.1 The final parameters for the best BGPAR fit. . . . . . . . . . . . . . . . 81

5.1 Raw number of events in neutrino energy bins passing flux cuts for neutrinos and anti-neutrinos. . . . . . . . . . . . . . . . . 90

5.2 Raw number of events in neutrino energy bins passing total cross section cuts for neutrinos and anti-neutrinos. . . . . . . . . . . . . . . . . . 104

5.3 Neutrino and anti-neutrino total cross sections for all energy bins in units $10^{-38} \mathrm{~cm}^{2} / \mathrm{GeV}$. Both the statistical and the systematic errors are shown for each bin excluding the normalization uncertainty. . . . . . . . . . . . 108

5.4 Anti-neutrino to neutrino total cross section ratio for all energy bins. Both the statistical and the systematic errors are shown for each bin. . . . . . . . . 109

6.1 Bin limits for $E_{\nu}, x$ and $y \ldots \ldots \ldots \ldots \ldots \ldots \ldots$

$7.1 Q^{2}$ bin limits and centers given in $\log _{10} Q^{2}(\mathrm{GeV}) \ldots \ldots \ldots$. . . . . . . 136 
$7.2 F_{2}$ and $x F_{3}$ values from simultaneous two parameter fit. Correlation between the structure functions are also provided. The quoted uncertainty includes both the statistical and systematic uncertainties. . . . . . . . . . . . . . . . 142

$7.3 \quad F_{2}$ and $x F_{3}$ values from simultaneous two parameter fit. Correlation between the structure functions are also provided. The quoted uncertainty includes both the statistical and systematic uncertainties. . . . . . . . . . . . . . 143

$7.4 F_{2}$ and $x F_{3}$ values from simultaneous two parameter fit. Correlation between the structure functions are also provided. The quoted uncertainty includes both the statistical and systematic uncertainties. . . . . . . . . . . . . . . . 144

$7.5 \quad F_{2}$ and $x F_{3}$ values from simultaneous two parameter fit. Correlation between the structure functions are also provided. The quoted uncertainty includes both the statistical and systematic uncertainties. . . . . . . . . . . . . . 145 


\section{LIST OF FIGURES}

1.1 DIS is the lepton scattering off the nucleon mediated via one of the electroweak gauge bosons. . . . . . . . . . . . . . . . . . . . 5

1.2 Neutrino charge current scattering Feynman diagram. . . . . . . . . . . 8

1.3 Helicity configurations allowed by the V-A structure of the weak interaction. Small arrows indicate the spin of the particles and large arrows, the momenta. 12

1.4 LO QCD diagrams. . . . . . . . . . . . . . . . . . 18

1.5 Feynman diagram for scattering from multiple partons. . . . . . . . . . . . . 22

2.1 A schematic drawing of the $\mathrm{NuTeV}$ beamline layout at Fermilab. . . . . . . . 24

2.2 Edge view of the SSQT. . . . . . . . . . . . . . . . . . 25

2.3 Flux prediction from beam Monte Carlo for neutrino (top) and anti-neutrino (bottom) mode. . . . . . . . . . . . . . . . . 27

2.4 Beam timing structure. . . . . . . . . . . . . . . . . . . 27

2.5 A schematic drawing of the test beam layout. . . . . . . . . . . . . . 28

2.6 The $\mathrm{NuTeV}$ detector. . . . . . . . . . . . . . . . . . . . . . . . . . . . . 29

2.7 A schematic drawing of one target unit. . . . . . . . . . . . . . . . . . 30

2.8 Front view (top) and cross section (bottom) of a scintillation counter. . . . . 33

2.9 Top view of a drift chamber (top) and cross section of three-wire DC (bottom). 34

2.10 Schematic drawing of the cross section view of the toroid. The coil marked with '*) was shunted to ground early in the run and disconnected. . . . . . . 35

2.11 Schematic drawing of the side view of the toroid. Drift chamber layout is shown. 36

$2.12 B_{\phi}$ component of the magnetic field in units of $\mathrm{GeV} / \mathrm{c}$ after calibration. . . . 37

2.13 A schematic drawing of the PMT read-out electronics. . . . . . . . . . . . . . 40 
3.1 Average counter response to muons as function of the position. . . . . . . .

3.2 The calibration fit to test beam hadrons, which determines the non-linearity. The point at $75 \mathrm{GeV}$ is adjusted to $1.000 \pm 0.001 \ldots \ldots \ldots$. . . . . . . 46

3.3 The width of the Poisson-like response function as a function of energy, obtained from a fit to the detector response for test beam hadrons. . . . . . . .

3.4 Fitted mean position at the front face of the toroid for all 45 muon test beam runs.

3.5 Detector response to test beam muons with incident energy of $100 \mathrm{GeV}$ (points)

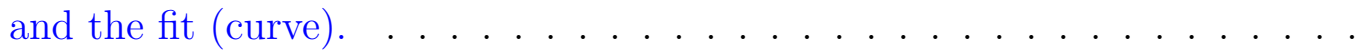

3.6 Energy dependence of the width of the leading Gaussian. The $\mathrm{x}$ axis is the mean muon energy $\left[p_{\mu}^{F F}\right]$ at the front face of the toroid. . . . . . . . . 57

4.1 Diagrams for QED radiative corrections for CC neutrino scattering. . . . . . 75

4.2 1-loop QED radiative corrections for charged current neutrino (red solid curve) and anti-neutrino (blue dashed curve) interactions for incident energy $E=45$ $\mathrm{GeV}$ for various x values. . . . . . . . . . . . . . . . . . .

4.3 1-loop QED radiative corrections for charged current neutrino (red solid curve) and anti-neutrino (blue dashed curve) interactions for incident energy $\mathrm{E}=95$ $\mathrm{GeV}$ for various x values.

4.4 1-loop QED radiative corrections for charged current neutrino (red solid curve) and anti-neutrino (blue dashed curve) interactions for incident energy $E=245$ $\mathrm{GeV}$ for various x values. . . . . . . . . . . . . . . . . . . .

4.5 Quark distributions calculated using our final BG model parameters at the initial $Q_{0}^{2}=12.6 \mathrm{GeV}$.

5.1 B/A for neutrinos (top) and anti-neutrinos (bottom) calculated with two different PDF sets. NuTeV BG model (red solid line) and GRV98 with Higher Twist (blue dashed line). . . . . . . . . . . . . . . . . . . .

5.2 Comparison for $\frac{d N}{d \nu}$ from data (blue circles), Monte Carlo smeared (red line) and Monte Carlo generated (light blue line) for $E=75 \mathrm{GeV}$ for neutrinos (top) and anti-neutrinos (bottom). . . . . . . . . . . . . 
$5.3 R_{\text {TERM }}$ calculated using to different PDF set. NuTeV BG model (red solid line) and the model from reference [33] (blue dashed line) . . . . . . . . . .

5.4 Physics corrections applied to the flux sample as function of $E_{\nu}$ for neutrinos (top) and anti-neutrinos (bottom) . . . . . . . . . . . . . . 94

5.5 Differential cross section $d \sigma / d \nu$ as function of $\nu$ for $E=60 \mathrm{GeV}$. . . . . . . 96

5.6 B/A fit to data for energy bin $(\mathrm{E}=75 \mathrm{GeV})$ for neutrinos (top) and antineutrinos (bottom). Since the $\nu$-dependence for DIS is different from that of quasi-elastics and resonances the fit is performed for data above $5 \mathrm{GeV}$. The blue dashed line shows the slope without scaling violations correction. . . . .

5.7 $\mathrm{B} / \mathrm{A}$ fit value as function of energy. $B / A$ is flat with energy for both neutrinos and anti-neutrinos. $\langle B / A\rangle^{\nu}=-0.34 \pm 0.04$ for neutrinos. $\langle B / A\rangle^{\bar{\nu}}=-1.68 \pm$ 0.03 for anti-neutrinos. . . . . . . . . . . . . . . . . .

5.8 Correction applied to neutrino (red solid line) and anti-neutrino (blue dashed line) fluxes to account for the energy dependence. . . . . . . . . . . . . . . . 100

5.9 Relative flux for neutrinos (red circles) and anti-neutrinos (blue circles). Systematic errors are included in quadrature. . . . . . . . . . . . . . . . . . . . 101

5.10 Isoscalar correction applied to the total neutrino (solid red curve) and antineutrino (dashed blue curve) cross sections as functions of $E_{\nu}$. . . . . . . . 105

5.11 Total isoscalar neutrino (circles) and anti-neutrino (squares) cross section for scattering off an iron target. Data is shown with its statistical uncertainty. The yellow band shows the systematic uncertainty (excluding the flux normalization uncertainty. The lines represent $2.1 \%$ uncertainty of the world average cross section. . . . . . . . . . . . . . . . . . . . . 107

5.12 The ratio of anti-neutrino to neutrino total cross sections (blue circles). The world average value (light blue line) $0.499 \pm 0.007$ is compared with the $\mathrm{NuTeV}$ average (red line) $0.505 \pm 0.0015$ (stat) \pm 0.0029 (syst) for the same energy range 30-200 GeV. Systematic errors are included. . . . . . . . . . . . . . . . . . . 110

6.1 Acceptance (red solid curve) for $\mathrm{E}=65 \mathrm{GeV}$ in $\mathrm{x}$ bins as a function of $\mathrm{y}$ for neutrinos (solid red curve) and anti-neutrinos (dashed blue curve). The minimum acceptance of $20 \%$ is shown with blue dashed line. . . . . . . . . . . . . . . 114 
6.2 Acceptance (red solid curve) for $\mathrm{E}=110 \mathrm{GeV}$ in $\mathrm{x}$ bins as a function of $\mathrm{y}$ for neutrinos (solid red curve) and anti-neutrinos (dashed blue curve). The minimum acceptance of $20 \%$ is shown with blue dashed line. . . . . . . . 115

6.3 Bin centering correction for neutrinos and anti-neutrinos with $\mathrm{E}=55 \mathrm{GeV}$. . 116

6.4 Bin centering correction for neutrinos anti-neutrinos with $\mathrm{E}=110 \mathrm{GeV}$. . . . 117

6.5 A schematic drawing of the iteration procedure used for cross section extraction.118

6.6 NuTeV final cross section and the final cross section model fit for $\mathrm{E}=55 \mathrm{GeV}$ in $x$ bins increasing from top to bottom as function of $y$ for neutrinos (left) and anti-neutrinos (right). . . . . . . . . . . . . . . 120

6.7 NuTeV final cross section and the final cross section model fit for $\mathrm{E}=110 \mathrm{GeV}$ in $x$ bins increasing from top to bottom as function of $y$ for neutrinos (left) and anti-neutrinos (right). . . . . . . . . . . . . . . . 121

6.8 NuTeV final cross section and the final cross section model fit for $\mathrm{E}=245 \mathrm{GeV}$ in $x$ bins increasing from top to bottom as function of $y$ for neutrinos (left) and anti-neutrinos (right). . . . . . . . . . . . . . 122

6.9 Monte Carlo compared with data for the muon energy, the hadron energy and muon angle for neutrinos(top three) and anti-neutrinos (bottom three). The yellow band represents the systematic uncertainty. . . . . . . . . . . . .

6.10 Differential cross sections in $x$ bins for neutrinos (left) and anti-neutrinos (right) at $E=65 \mathrm{GeV}$. Points are $\mathrm{NuTeV}$ (filled circles), CCFR (open squares), and CDHSW (crosses). Error bars show statistical and systematic errors in quadrature. Solid curve shows fit to $\mathrm{NuTeV}$ data. $(x=0.08,0.225,0.45$, and 0.75 bins are not shown). . . . . . . . . . . . . . . . 127

6.11 Differential cross sections in $x$ bins for neutrinos (left) and anti-neutrinos (right) at $E=150 \mathrm{GeV}$. Points are $\mathrm{NuTeV}$ (filled circles), CCFR (open squares), and CDHSW (crosses). Error bars show statistical and systematic errors in quadrature. Solid curve shows fit to NuTeV data. $(x=0.08,0.225$, 0.45 , and 0.75 bins are not shown). 
6.12 Differential cross sections in $x$ bins for neutrinos (left) and anti-neutrinos (right) at $E=245 \mathrm{GeV}$. Points are NuTeV (filled circles), CCFR (open squares). (CDHSW does not have data above $190 \mathrm{GeV}$ ). Error bars show statistical and systematic errors added in quadrature. Solid curve shows fit to NuTeV data. $(x=0.08,0.225,0.45$, and 0.75 bins are not shown). . . . . .

6.13 Point-to-point ratio of $\mathrm{CCFR}$ and $\mathrm{NuTeV}$ cross sections weight averaged over $\mathrm{E}$ and $\mathrm{y}$ as function of $\mathrm{x}$ for neutrinos (top) and anti-neutrinos(bottom). . . .

6.14 Ratio of CCFR and NuTeV magnetic field maps as function of the (x,y) position. The ratio is adjusted to have value 1.0 at the position where both $\mathrm{NuTeV}$ and CCFR have high precision calibration beams. . . . . . . . . . . . 132

7.1 NuTeV measurement of $F_{2}\left(x, Q^{2}\right)$ (solid circles) compared with previous $\nu$-Fe results; CCFR (open circles) and CDHSW (triangles). The data are corrected to an isoscalar (iron) target and for QED radiative effects as described in the text. The curve show the NuTeV model. . . . . . . . . . . . . . . . 138

7.2 NuTeV measurement of $x F_{3}\left(x, Q^{2}\right)$ (solid circles) compared with previous $\nu$-Fe results; CCFR (open circles) and CDHSW (triangles). The data are corrected to an isoscalar (iron) target and for QED radiative effects as described in the text. The curve show the NuTeV model.

$7.3 F_{2}\left(x, Q^{2}\right)$ fractional difference $\frac{F_{2}-F_{2}^{T R V F S}}{F_{2}^{T R V F S}}$ with respect to the TRVFS(MRST2001E) model. Data points are NuTeV (solid dots) and CCFR (open circles). Theory curves are ACOTFFS(CTEQ5HQ1) (solid line) and TRVFS(MRST2001E) $\pm 1 \sigma$ (dashed lines). Theory curves are corrected for target mass and nuclear effects. . . . . . . . . . . . . . . . . . . . 147

$7.4 x F_{3}\left(x, Q^{2}\right)$ fractional difference $\frac{x F_{3}-x F_{3}^{T R V F S}}{x F_{3}^{T R V F S}}$ with respect to the TRVFS(MRST2001E) model. Data points are $\mathrm{NuTeV}$ (solid dots) and CCFR (open circles). Theory curves are ACOTFFS(CTEQ5HQ1) (solid line) and TRVFS(MRST2001E) $\pm 1 \sigma$ (dashed lines). Theory curves are corrected for target mass and nuclear effects. . . . . . . . . . . . . . . . . . . 148 
$7.5 F_{2}\left(x, Q^{2}\right)$ (top) and $x F_{3}$ (bottom) fractional difference with respect to the TRVFS(MRST2001E) model. Data points are NuTeV (solid dots) and CCFR (open circles). Red curves represents the TRVFS(MRST2001E) with the nuclear correction from reference [43] instead of the standard nuclear correction from charged lepton data. Theory curves are corrected for target mass effects. 149

7.6 Comparison between the QCD logslopes for NuTeV, CCFR, CDHSW and two NLO QCD theory predictions TRVFS(MRST2001E) and ACOTFFS(CTEQ5HQ1).150

7.7 Comparison between the QCD logslopes for NuTeV, CCFR, CDHSW and two NLO QCD theory predictions TRVFS(MRST2001E) and ACOTFFS(CTEQ5HQ1).151 


\subsection{INTRODUCTION}

Deep inelastic scattering (DIS), the scattering of a high energy lepton off a quark inside a nucleon, has been a proving ground for QCD, the theory of strong interactions. Chargedleptons and neutrinos have been used to measure parton densities and their QCD evolution with high-precision over a wide range in $Q^{2}$. Uniquely, neutrino DIS, via the weak interac-

tion probe, allows simultaneous measurement of two structure functions: $F_{2}\left(x, Q^{2}\right)$ and the parity-violating structure function, $x F_{3}\left(x, Q^{2}\right)$.

This thesis presents a new measurement of high-energy neutrino and anti-neutrino differential cross sections from high-statistics data samples. Previous high-statistics measurements of the neutrino and anti-neutrino differential cross section have been reported [1], [2]. This experiment has two improvements: first, a sign-selected beam allowed separate neutrino and anti-neutrino running and, second, a calibration beam continuously measured the detector's response. The largest experimental uncertainties in previous measurements arose from knowledge of energy scale and detector response functions [3], [4]. NuTeV addressed this by using a dedicated calibration beam of hadrons, electrons and muons that alternated with neutrino running once every minute throughout the one year data-taking peroid.

NuTeV's other improvement was separate neutrino and anti-neutrino running. NuTeV ran in two modes, $(\nu$ and $\bar{\nu})$, with the muon spectrometer polarity always set to focus the primary charged-lepton from the interaction vertex (e.g. $\mu^{-}$in $\nu$-mode or $\mu^{+}$in $\bar{\nu}$-mode). In determining the charged-current differential cross sections, this allowed better and more uniform acceptance in the two running modes and removed ambiguity in the muon sign determination present in a mixed $\nu$ and $\bar{\nu}$ beam.

The first chapter will introduce the Standard Model of particle physics, and will present the theoretical framework for deep inelastic scattering. The remainder of this thesis is 
organized as follows:

- Chapter 2 describes the neutrino beamline (SSQT) and detector.

- Chapter 3 describes the calibration of the detector, data samples and event selection.

- Chapter 4 provides a detailed description of the event simulator including the neutrino cross section model and detector response model.

- Chapter 5 presents the method for extraction of the flux and total neutrino cross section.

- Chapter 6 presents the method for extraction of the differential cross section and comparisons with previous measurements.

- Chapter 7 presents the measurement of the structure functions, $F_{2}$ and $x F_{3}$, and comparisons with previous neutrino measurements. Results are also compared with theoretical models

- Chapter 8 gives final remarks and conclusions.

\subsection{THE STANDARD MODEL}

Particle physics, both theoretical and experimental, studies the elementary particles and their interactions. The contemporary view is that all matter is built from a number of fundamental particles, which are considered structureless. These fundamental particles can be divided into two major groups; fermions and bosons. Most simplistically the fermions are the building blocks and the bosons mediate the interactions among the fermions. All matter is composed of these fundamental particles. The atom consists of electrons and a nucleus, which contains protons and neutrons (nucleons). These nucleons are composed of quarks. The proton consists of two up and one down quarks, and the neutron consists of one up and two down quarks.

We can divide the fermions into two groups based on the interactions in which they participate: quarks and leptons. Quarks participate in all interactions (Table 1.3). Leptons participate in the gravitational, weak, and electromagnetic (only the ones which carry electric charge) interactions. Both quarks and leptons are divided in three "generations" of doublets, which gives six varieties (flavors) of quarks and leptons. The six quarks flavors are up (u), 


\begin{tabular}{|c|c|c|c|}
\hline Electric Charge & I Generation & II Generation & III generation \\
\hline$\frac{2}{3}$ & $\mathbf{u}$ & $\mathbf{c}$ & $\mathbf{t}$ \\
\hline$-\frac{1}{3}$ & $\mathbf{d}$ & $\mathbf{s}$ & $\mathbf{b}$ \\
\hline
\end{tabular}

Table 1.1: Quarks are ordered in three generations. Electric charge is given as a fraction of the electric charge of the proton.

down (d), strange (s), charm (c), bottom (b) and top (t). Table 1.1 shows the ordering of the quarks into generations. The electric charge of the quarks is given as a fraction of the electric charge of the proton. The electron $\left(e^{-}\right)$, the muon $\left(\mu^{-}\right)$and the tau $\left(\tau^{-}\right)$are the leptons carrying electric charge. All neutrinos, which include the electron neutrino $\nu_{e}$, the muon neutrino $\nu_{\mu}$ and the tau neutrino $\nu_{\tau}$, are electrically neutral leptons. Table 1.2 shows the leptons and their properties.

The second part of the picture is "anti-matter", which consists of the particles which are the charge-conjugates of the quarks and leptons we already described. The charge-conjugate for each fermion has the same mass, but opposite charges.

There are four known interactions through which fundamental particles interact with each other. Table 1.3 lists the four known forces which govern the interactions among the fundamental particles: strong, electromagnetic, weak and gravitational. Particles interact by exchanging gauge bosons. All fermions interact through the weak interaction, which is mediated via the massive gauge bosons $Z_{0}, W^{ \pm}$. All fundamental particles carrying electric charge interact through the electromagnetic interaction, which is mediated by the photon $(\gamma)$. Only quarks interact through the strong interaction, because they are the only fermions carrying color charge (the strong charge) of which there are three types. The strong interaction is mediated by a spin-1 gauge boson, gluon, which also carries color charge. This unique property of the gluons allows them to interact with each other. The Standard Model(SM) of particle physics unifies three of the four known interactions: strong, electromagnetic and weak. Gravity does not easily fit this unification. However, the gravitational force is negligible 


\begin{tabular}{|c|c|c|c|}
\hline Electric Charge & I Generation & II Generation & III generation \\
\hline 0 & $\nu_{\mathbf{e}}$ & $\nu_{\mu}$ & $\nu_{\tau}$ \\
\hline-1 & $\mathbf{e}$ & $\mu$ & $\tau$ \\
\hline
\end{tabular}

Table 1.2: Leptons are ordered in three generations. Electric charge is given as a fraction of the electric charge of the proton.

when interactions between elementary particle are considered.

The Standard Model is accurate at describing known particle interactions. It explains all existing data and is consistent with results from all subfields of particle physics. However, there are a number of more fundamental questions for which the Standard model offers no explanation. For example, it can't explain the origin of mass, the values of the coupling constants, the number of fermions or the hierarchy between the generations of quarks and leptons. Perhaps an elegant theory exists "beyond" the Standard Model which will give answers to all these questions.

\begin{tabular}{|c|c|c|c|}
\hline Interaction & Participating particles & Mediating boson & Relative coupling \\
\hline Strong & Quarks & gluon $(g)$ & $>1$ \\
\hline Electromagnetic & Quarks and charged leptons & photon $(\gamma)$ & $\sim 1 / 137$ \\
\hline Weak & All & $Z_{0}, W^{ \pm}$ & $10^{-5}$ \\
\hline Gravitational & All & Graviton & $10^{-42}$ \\
\hline
\end{tabular}

Table 1.3: Fundamental interactions and their relative strengths. 


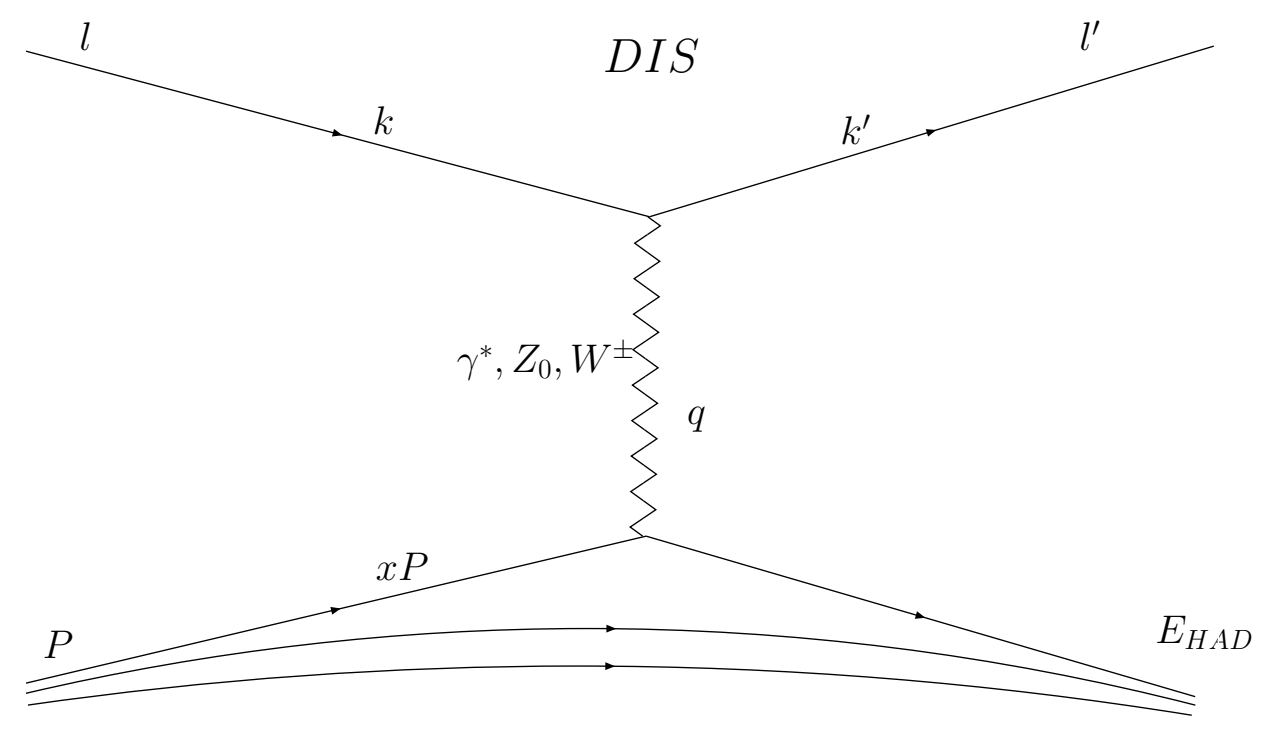

Figure 1.1: DIS is the lepton scattering off the nucleon mediated via one of the electroweak gauge bosons.

\subsection{DEEP INELASTIC SCATTERING}

Deep inelastic scattering (DIS) is the process in which a lepton interacts with a quark in nucleon producing a lepton, and a hadronic system in the final state. This can be written as:

$$
l+N \rightarrow l^{\prime}+X
$$

where $l$ is the initial state lepton, $N$ is the nucleon, $l^{\prime}$ is the final state lepton, and $X$ is a final hadronic state. The incident lepton scatters inelastically of a quark in the nucleon via exchange of a vector boson $\left(\gamma^{*}, W^{ \pm}\right.$or $\left.Z_{0}\right)$. The excited nucleon fragments (turns into hadrons) and together with remnant quarks comprises the hadronic final state, composed of hadronic particles (protons, neutrons, pions, kaons, etc.). 


\subsubsection{Kinematics of DIS}

Figure 1.1 shows the tree level Feynman diagram for the DIS interaction, where $k$ is the four-momentum of the incident lepton, $k^{\prime}$ and $\theta_{l^{\prime}}$ are the four-momenta and the angle of the outgoing lepton, $P$ is the four-momentum of the nucleon, and $q$ is the four-momentum of the virtual gauge boson. In the laboratory frame these vectors have the form

$$
\begin{aligned}
k & =\left(E_{l}, \vec{p}_{l}\right), \\
k^{\prime} & =\left(E_{l^{\prime}}, \vec{p}_{l^{\prime}}\right), \\
p & =(M, 0,0,0), \\
q & =(\nu, \vec{q}), \\
p^{\prime} & =p+q=p+\left(k-k^{\prime}\right),
\end{aligned}
$$

where $E_{l}, \vec{p}_{l}$ and $E_{l^{\prime}}, \vec{p}_{l^{\prime}}$ are the energies and momenta of the incident lepton, $l$, and the outgoing lepton, $l^{\prime}$, and $M$ is the mass of the nucleon.

We can define the following invariant kinematic quantities evaluated in terms of the lab frame variables:

- $Q^{2}$ is the negative of the four-momentum

$$
Q^{2}=-q^{2}=-\left(k-k^{\prime}\right)^{2}=m_{l^{\prime}}^{2}+2 E_{l}\left(E_{l^{\prime}}-p_{l^{\prime}} \cos \theta_{l^{\prime}}\right) .
$$

- $\nu$ is the energy transferred to the hadronic system

$$
\nu=\frac{p \cdot q}{M}=E_{l}-E_{l^{\prime}}=E_{H A D} .
$$

- $W^{2}$ is the squared invariant mass of the hadronic system

$$
W^{2}=(p+q)^{2}=M^{2}+2 M \nu-Q^{2} .
$$


- $y$ is the inelasticity, or the fraction of energy of the incident lepton transferred to the hadronic system

$$
y=\frac{p \cdot q}{p \cdot k}=\frac{E_{H A D}}{E_{l}} .
$$

$y$ can also be expressed in terms of the center of mass (CM) frame muon scattering angle, $\theta^{*}$, as

$$
y \simeq 1-\frac{1}{2}\left(1+\cos \theta^{*}\right)
$$

- $x$ is the Bjorken rescaling variable (introduced by Bjorken [5]), which represents the fraction of the nucleon momentum carried by the struck quark

$$
x=\frac{-q^{2}}{2 p \cdot q}=\frac{Q^{2}}{2 M \nu} .
$$

\subsubsection{Neutrino-Nucleon Charge Current Differential Cross Section}

In neutrino charged-current (CC) DIS the incident lepton is a neutrino which scatters off a quark in the nucleon by exchanging the charged gauge boson $W^{+(-)}$. The final state includes a charged lepton with the same lepton number and hadrons produced in the hadronic final state (Figure 1.2). An example of such a process is

$$
\nu_{\mu}\left(\bar{\nu}_{\mu}\right)+N \longrightarrow \mu^{-}\left(\mu^{+}\right)+X
$$

where a muon neutrino scatters from a nucleon and produces a muon in the final state. The differential cross section for neutrino DIS can be derived from the matrix element

$$
\mathcal{M}=\frac{G_{F}}{\sqrt{2}} \frac{1}{1+\frac{Q^{2}}{M_{W}^{2}}} \bar{u}_{\mu} \gamma_{\alpha}\left(1-\gamma_{5}\right) u_{\nu}<X\left|J_{C C}\right| N ; p, s>,
$$

where $G_{F}$ is the Fermi coupling constant, $M_{W}$ is the mass of $W^{ \pm}, \bar{u}_{\mu} \gamma_{\alpha}\left(1-\gamma_{5}\right) u_{\nu}$ is the leptonic current and $<X\left|J_{C C}\right| N ; p, s>$ is the hadronic current. Hadrons are not point-like particles, hence they can not be described with a current similar to the leptonic current. Summing over all lepton and hadron states the differential cross section can be written as:

$$
\frac{d^{2} \sigma_{C C}^{\nu}}{d \Omega_{\mu} d E_{\mu}}=\frac{G_{F}^{2}}{2\left(1+\frac{Q^{2}}{M_{W}^{2}}\right)^{2}} \frac{m_{\nu} m_{\mu}}{E_{\nu}} \frac{E_{\mu}}{4 \pi^{2}} L_{\alpha \beta} W^{\alpha \beta},
$$




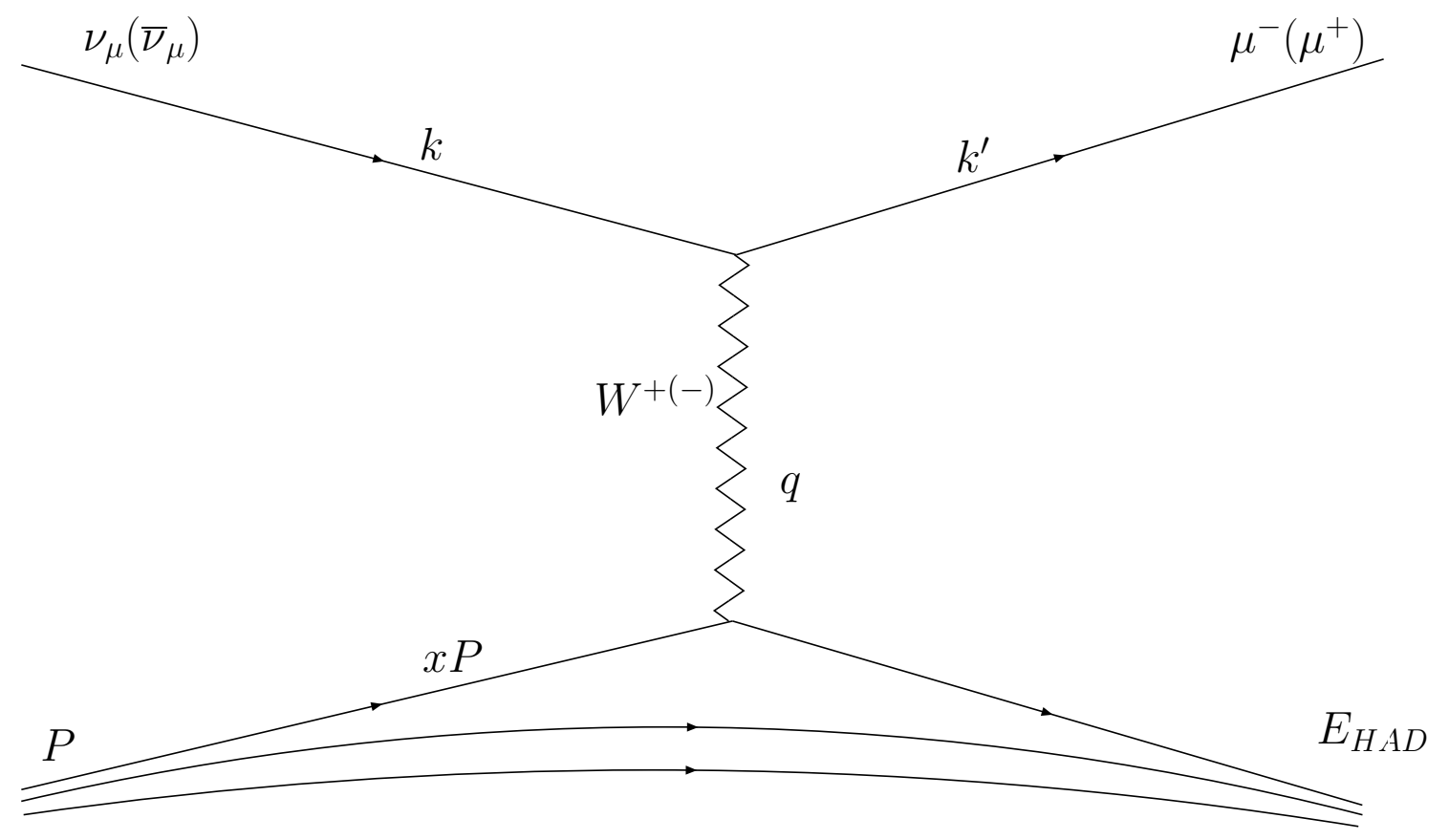

Figure 1.2: Neutrino charge current scattering Feynman diagram.

where $L_{\alpha \beta}$ is the leptonic tensor and $W^{\alpha \beta}$ is the hadronic tensor. The leptonic tensor can be written in terms of the energy-momentum four-vectors of the leptons participating in the interaction

$$
\begin{aligned}
L_{\alpha \beta} & =\sum_{s, s^{\prime}} \bar{u}_{\mu}\left(k^{\prime}, s^{\prime}\right) \gamma_{\alpha}\left(1-\gamma_{5}\right) u_{\nu}(k, s) \bar{u}_{\nu}(k, s) \gamma_{\beta}\left(1-\gamma_{5}\right) u_{\mu}\left(k^{\prime}, s^{\prime}\right) \\
& =\frac{2}{m_{\mu} m_{\nu}}\left[k_{\alpha}^{\prime} k_{\beta}+k_{\beta}^{\prime} k_{\alpha}-k \cdot k^{\prime} g_{\alpha \beta} \mp i k^{\gamma} k^{\prime \delta} \varepsilon_{\gamma \delta \alpha \beta}\right] .
\end{aligned}
$$

The last term (which is parity violating; '-' for $\nu$, '+' for $\bar{\nu}$ ) is specific for the weak gauge boson exchange. The leptonic current for virtual photon exchange does not have this term. The hadronic tensor is parameterized in the most general form for a second order tensor constructed from the four-vectors $q$ and $p$

$$
\begin{aligned}
W^{\alpha \beta} & =-g^{\alpha \beta} W_{1}+\frac{p^{\alpha} p^{\beta}}{M^{2}} W_{2}-i \frac{\varepsilon^{\alpha \beta \gamma \delta} p_{\gamma} p_{\delta}}{2 M^{2}} W_{3} \\
& +\frac{q^{\alpha} q^{\beta}}{M^{2}} W_{4}+\frac{p^{\alpha} q^{\beta}+p^{\beta} q^{\alpha}}{M^{2}} W_{5}+i \frac{p^{\alpha} q^{\beta}-p^{\beta} q^{\alpha}}{2 M^{2}} W_{6}
\end{aligned}
$$


where $g^{\alpha \beta}$ is the metric tensor of Minkowski space. The hadronic current is conserved $\partial_{\mu} J^{\mu}=0$, which can be written as

$$
q^{\alpha} W_{\alpha \beta}=q^{\beta} W_{\alpha \beta}=0
$$

Contracting the leptonic and hadronic tensors, neglecting the terms proportional to the lepton mass, and using general invariance arguments the differential cross section can be written as

$$
\begin{aligned}
\frac{d^{2} \sigma^{\nu(\bar{\nu}) N}}{d x d y} & =\frac{G_{F}^{2} M E}{\pi\left(1+\frac{Q^{2}}{M_{W}^{2}}\right)^{2}}\left[\frac{y^{2} M}{2} 2 x W_{1}\left(\nu, Q^{2}\right)\right. \\
& \left.+\left(1-y-\frac{M x y}{2 E}\right) \nu W_{2}\left(\nu, Q^{2}\right) \pm y\left(1-\frac{y}{2}\right) \nu x W_{3}\left(\nu, Q^{2}\right)\right],
\end{aligned}
$$

where $+(-)$ the last term is for neutrino (antineutrino) interactions. The change of variables $\left(\Omega_{\mu}, E_{\mu}\right) \rightarrow(x, y)$ was performed using the Jacobian form

$$
d \Omega_{\mu} d E_{\mu}=\frac{2 \pi M \nu}{E_{\mu}} d x d y
$$

The structure functions $W_{1}, W_{2}, W_{3}$ can be transformed to dimensionless quantities

$$
\begin{aligned}
& F_{1}\left(x, Q^{2}\right)=M W_{1}\left(\nu, Q^{2}\right), \\
& F_{2}\left(x, Q^{2}\right)=\nu W_{2}\left(\nu, Q^{2}\right), \\
& F_{3}\left(x, Q^{2}\right)=\nu W_{3}\left(\nu, Q^{2}\right) .
\end{aligned}
$$

The differential cross section in terms of the dimensionless structure functions $2 x F_{1}, F_{2}$ and $x F_{3}$ is

$$
\begin{aligned}
\frac{d^{2} \sigma^{\nu(\bar{\nu}) N}}{d x d y} & =\frac{G_{F}^{2} M E}{\pi\left(1+\frac{Q^{2}}{M_{W}^{2}}\right)^{2}}\left[\frac{y^{2}}{2} 2 x F_{1}\left(x, Q^{2}\right)\right. \\
& \left.+\left(1-y-\frac{M x y}{2 E}\right) F_{2}\left(x, Q^{2}\right) \pm y\left(1-\frac{y}{2}\right) x F_{3}\left(x, Q^{2}\right)\right] .
\end{aligned}
$$

All of the information about the structure of the nucleon is encoded in the structure functions $2 x F_{1}, F_{2}$ and $x F_{3}$. 
We can express the structure functions in terms of the cross sections for absorbing transversely and longitudinally polarized $\mathrm{W}$ bosons, $\sigma_{T}$ and $\sigma_{L}$, respectively

$$
\begin{aligned}
& F_{1}=\frac{M K}{\pi G_{F} \sqrt{2}} \sigma_{T}, \\
& F_{2}=\frac{\nu K}{\pi G_{F} \sqrt{2}} \frac{Q^{2}}{Q^{2}+\nu^{2}}\left(\sigma_{T}+\sigma_{L}\right),
\end{aligned}
$$

where $\mathrm{K}$ represents the flux of virtual bosons. The ratio of the longitudinal and transverse cross sections is

$$
R\left(x, Q^{2}\right)=\frac{\sigma_{L}}{\sigma_{T}}=\frac{F_{2}}{2 x F_{1}}\left(1+\frac{\nu^{2}}{Q^{2}}\right)-1
$$

\subsection{QUARK PARTON MODEL}

The naive parton model [6] offers an intuitive picture of the sub-structure of the nucleon. It assumes that the nucleon consists of non-interacting point-like constituents called "partons" (quarks and gluons). The model is valid in the "infinite momentum" frame, in which the time scale of the interactions between partons (strong interaction) is much longer than the collision time. In this frame the partons are on-shell particles traveling collinearly with the nucleon and carrying a fraction, $\xi$, of nucleon's momentum. In the context of the parton model, neutrino-nucleon inelastic scattering becomes elastic scattering of two structureless on-shell particles. The final state four-momentum of the struck parton is

$$
P^{\prime}=\xi P+q,
$$

where $P$ is the momentum of the nucleon.

$$
P^{\prime 2}=\left(\xi^{2} P^{2}+q^{2}+2 \xi P \cdot q\right)
$$

Neglecting the mass of the parton $P^{\prime 2}$ and the term $\xi^{2} P^{2}$ yields

$$
\xi=\frac{-q^{2}}{2 P \cdot q}=x
$$


which can be identified as the Bjorken scaling variable $x$. The Bjorken scaling variable can be interpreted as the fraction of nucleon momentum carried by the struck parton. In the parton model the structure functions have the form:

$$
\begin{aligned}
2 M W_{1}^{\text {point }}\left(\nu, Q^{2}\right) & =\frac{Q^{2}}{2 M \nu} \delta\left(1-\frac{Q^{2}}{2 M \nu}\right) \\
\nu W_{2}^{\text {point }}\left(\nu, Q^{2}\right) & =\delta\left(1-\frac{Q^{2}}{2 M \nu}\right)
\end{aligned}
$$

In the limit $Q^{2} \rightarrow \infty$ the structure functions depend only on the dimensionless scaling variable $x$,

$$
\lim _{Q^{2} \rightarrow \infty} F_{i}\left(x, Q^{2}\right)=F_{i}(x)
$$

This behavior is known as Bjorken scaling [5].

Neutrinos and anti-neutrinos are highly relativistic fermions with spin- $\frac{1}{2}$, hence the neutrinos are left-handed and anti-neutrinos are right-handed. The V-A nature of the weak interaction imposes that neutrinos(antineutrinos) can interact only with left(right)-handed particles and right(left)-handed anti-particles. If we assume that the partons are fermions with spin $\frac{1}{2}$ then there are only two possible helicity configurations for neutrino scattering and two for antineutrino scattering (see Figure 1.3). The cross section for elastic scattering of neutrinos with partons can be derived from the requirement that angular momentum is conserved.

$$
\begin{aligned}
\frac{d^{2} \sigma^{\nu q}}{d \cos \theta^{*}} & =\frac{d^{2} \sigma^{\overline{\nu q}}}{d \cos \theta^{*}}=\frac{G^{2} M E_{\nu}}{\pi\left(1+\frac{Q^{2}}{M_{W}^{2}}\right)}, \\
\frac{d^{2} \sigma^{\nu \bar{q}}}{d \cos \theta^{*}} & =\frac{d^{2} \sigma^{\bar{\nu} q}}{d \cos \theta^{*}}=\frac{G^{2} M E_{\nu}}{\pi\left(1+\frac{Q^{2}}{M_{W}^{2}}\right)}\left(\frac{1+\cos \theta^{*}}{2}\right)^{2}, \\
\frac{d^{2} \sigma^{\nu k}}{d \cos \theta^{*}} & =\frac{d^{2} \sigma^{\bar{\nu} k}}{d \cos \theta^{*}}=\frac{G^{2} M E_{\nu}}{\pi\left(1+\frac{Q^{2}}{M_{W}^{2}}\right)}\left(\frac{\cos \theta^{*}}{2}\right)^{2}
\end{aligned}
$$

The cross section depends only on the CM energy, $s=2 M E$, and the angle of the outgoing lepton in the $\mathrm{CM}$ frame, $\theta^{*}$. Equation 1.23 corresponds to the case with total spin $S=0$, hence there is no preferred direction in the space. Equation 1.24 corresponds to the case with total spin of the system $S=1$. The cross section in this case is suppressed by the 


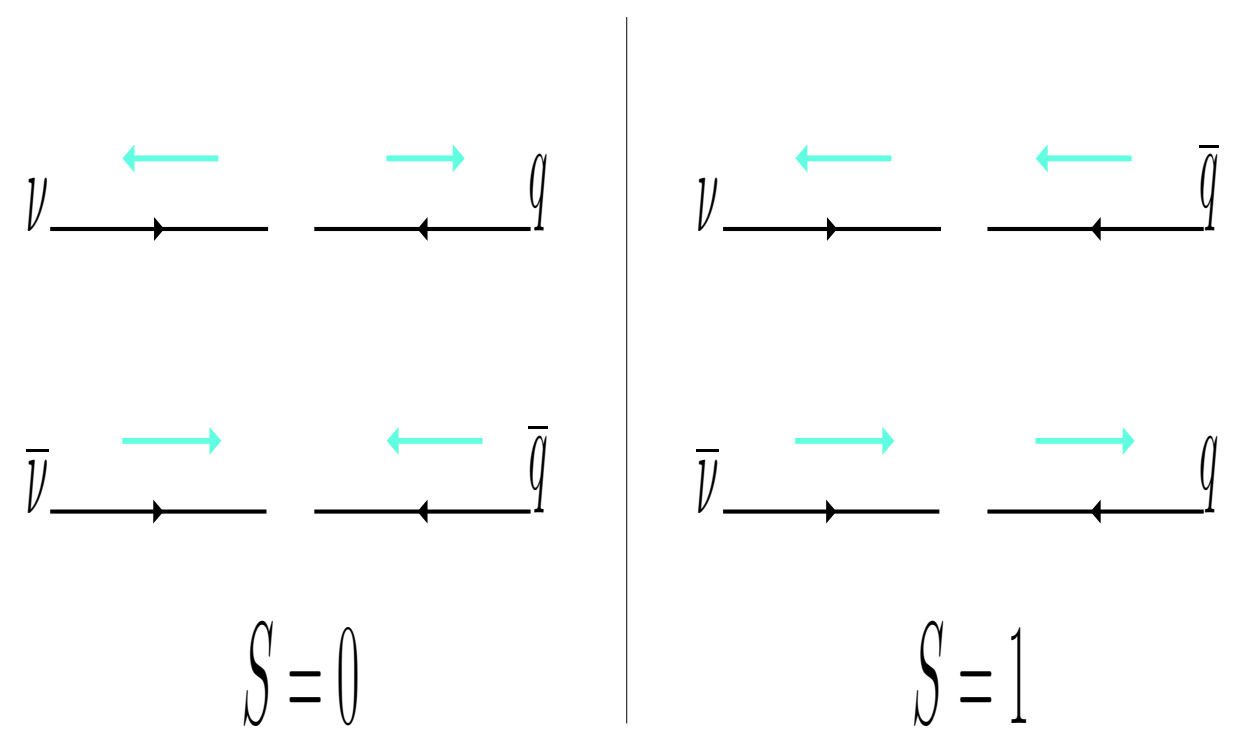

Figure 1.3: Helicity configurations allowed by the V-A structure of the weak interaction. Small arrows indicate the spin of the particles and large arrows, the momenta.

factor $\left(\frac{1+\cos \theta^{*}}{2}\right)^{2}$. If $\theta^{*}=\pi$ the cross section is 0 , because it violates conservation of the total angular momentum. Equation 1.25 corresponds to neutrino scattering off spin-0 particle.

The quark parton model (QPM) is the logical combination of the parton model and the static quark model. In the QPM the spin- $\frac{1}{2}$ partons are identified as quarks and anti-quarks. The QPM considers the nucleon to be constructed of 3 valence quarks, which give the nucleon its flavor and charge properties. The nucleon also contains "sea" quarks (quark-antiquark pairs), which contribute no net flavor or charge. We can express the quark and antiquark distributions as

$$
\begin{aligned}
& q(x)=q_{v}(x)+q_{s}(x) \\
& \bar{q}(x)=\bar{q}_{s}(x)=q_{s}(x) .
\end{aligned}
$$

The probability of finding a quark of type $q$ which carries a fraction of nucleon's momentum in the interval $(x, x+d x)$ is $q(x) d x$, where $q(x)$ is the probability distribution 
function. The differential cross section for neutrino scattering off a single quark of type $q$ with momentum distribution $x q(x)$ is

$$
\frac{d^{2} \sigma}{d x d y} \propto \frac{G_{F}^{2} M E}{\pi\left(1+\frac{Q^{2}}{M_{W}^{2}}\right)^{2}} x q(x) .
$$

Combining Equations 1.7, and 1.23-1.26 and accounting for all constituents in the nucleon we obtain the differential cross section for neutrino(anti-neutrino)-nucleon scattering

$$
\begin{aligned}
\frac{d^{2} \sigma^{\nu}}{d x d y} & =\frac{G^{2} x s}{\pi\left(1+\frac{Q^{2}}{M_{W}^{2}}\right)}\left[q(x)+\left(1-y^{2}\right) \bar{q}(x)+2(1-y) k(x)\right] \\
\frac{d^{2} \sigma^{\bar{\nu}}}{d x d y} & =\frac{G^{2} x s}{\pi\left(1+\frac{Q^{2}}{M_{W}^{2}}\right)}\left[\bar{q}(x)+\left(1-y^{2}\right) q(x)+2(1-y) k(x)\right]
\end{aligned}
$$

where the distribution $k(x)$ is the density of spin-0 particles. Comparing the $y$-dependence of Equations 1.18 and 1.27 yields the structure functions $2 x F_{1}, F 2$ and $x F_{3}$ in term of the parton densities

$$
\begin{aligned}
2 x F_{1}^{\nu(\bar{\nu})} & =2\left[x q^{\nu(\bar{\nu})}(x)+x \bar{q}^{\nu(\bar{\nu})}(x)\right], \\
F_{2}^{\nu(\bar{\nu})} & =2\left[x q^{\nu(\bar{\nu})}(x)+x \bar{q}^{\nu(\bar{\nu})}(x)+2 x k^{\nu(\bar{\nu})}(x)\right], \\
x F_{3}^{\nu(\bar{\nu})} & =2\left[x q^{\nu(\bar{\nu})}(x)-x \bar{q}^{\nu(\bar{\nu})}(x)\right],
\end{aligned}
$$

where $M x y / E$ term is neglected. If the scattering involves only spin- $\frac{1}{2}$ constituents we obtain the Callan-Gross relation [7]

$$
2 x F_{1}=F_{2} .
$$

However, the quarks can have a small transverse momentum $p_{T}$, which effectively gives them spin-0 behavior, in which case the Callan-Gross relation will be violated (see Section 1.4.3). 
Neutrino charge current scattering is mediated by the charged gauge bosons, $W^{ \pm}$. Conservation of the charge and lepton number at the quark vertex requires that neutrino chargedcurrent interactions involve only $d, s, \bar{u}$ and $\bar{c}$ quarks, while antineutrino charged-current scattering involves $u, c, \bar{d}$ and $\bar{s}$. Hence neutrino scattering is sensitive to the flavor of the nucleon's constituents. The quark distributions contributing to neutrino (antineutrino)-proton structure functions are

$$
\begin{array}{ll}
q^{\nu p}(x)=d^{p}(x)+s^{p}(x) & , \quad q^{\bar{\nu} p}(x)=u^{p}(x)+c^{p}(x), \\
\bar{q}^{\nu p}(x)=\bar{u}^{p}(x)+\bar{c}^{p}(x) & , \quad \bar{q}^{\bar{\nu} p}(x)=\bar{d}^{p}(x)+\bar{s}^{p}(x) .
\end{array}
$$

The strong isospin symmetry between the proton and the neutron gives the following symmetries between their quark distributions

$$
\begin{array}{ll}
d(x) \equiv d^{p}(x)=u^{n}(x) \quad, \quad \bar{d}(x) \equiv \bar{d}^{p}(x)=\bar{u}^{n}(x), \\
u(x) \equiv u^{p}(x)=d^{n}(x) \quad, \quad \bar{u}(x) \equiv \bar{u}^{p}(x)=\bar{d}^{n}(x) .
\end{array}
$$

We will also assume the following sea symmetries

$$
\begin{array}{ll}
s(x) \equiv s^{p}(x)=s^{n}(x) & , \quad \bar{s}(x) \equiv \bar{s}^{p}(x)=\bar{s}^{n}(x), \\
c(x) \equiv c^{p}(x)=c^{n}(x) & , \quad \bar{c}(x) \equiv \bar{c}^{p}(x)=\bar{c}^{n}(x) .
\end{array}
$$

Accounting for all symmetries, we express neutron quark distributions as

$$
\begin{array}{ll}
q^{\nu n}(x)=u(x)+s(x) \quad, \quad q^{\bar{\nu} n}(x)=d(x)+c(x), \\
\bar{q}^{\nu n}(x)=\bar{d}(x)+\bar{c}(x) \quad, \quad \bar{q}^{\bar{\nu} n}(x)=\bar{u}(x)+\bar{s}(x),
\end{array}
$$

which yields the quark distributions contributing to the neutrino(antineutrino)-nucleon structure functions. An isoscalar target consists of an equal number of protons and neutrons. Using Equations 1.30 and 1.31 we obtain the corresponding quark distributions for an isoscalar target

$$
\begin{aligned}
q^{\nu N}(x) & =\frac{1}{2}[u(x)+d(x)+2 s(x)], \\
q^{\bar{\nu} N}(x) & =\frac{1}{2}[u(x)+d(x)+2 c(x)], \\
\bar{q}^{\nu N}(x) & =\frac{1}{2}[\bar{u}(x)+\bar{d}(x)+2 \bar{c}(x)], \\
\bar{q}^{\bar{\nu} N}(x) & =\frac{1}{2}[\bar{u}(x)+\bar{d}(x)+2 \bar{s}(x)] .
\end{aligned}
$$


Finally, using 1.28 and 1.32 the neutrino(antineutrino)-nucleon structure functions $2 x F_{1}^{\nu(\bar{\nu})}$ and $F_{2}^{\nu(\bar{\nu})}$ for an isoscalar target are

$$
\begin{aligned}
2 x F_{1}^{\nu N} & =2 x F_{1}^{\bar{\nu} N}=x u+x \bar{u}+x d+x \bar{d}+2 x s+2 x c, \\
F_{2}^{\nu N} & =F_{2}^{\bar{\nu} N}=x u+x \bar{u}+x d+x \bar{d}+2 x s+2 x c,
\end{aligned}
$$

where the Callan-Gross relation 1.29 is assumed. Using Equation 1.28 and 1.32 we can express the parity-violating structure function, $x F_{3}$, in terms of the isoscalar quark distributions

$$
\begin{aligned}
& x F_{3}^{\nu N}=x u_{v}+x d_{v}+2 x s-2 x \bar{c} \\
& x F_{3}^{\bar{\nu} N}=x u_{v}+x d_{v}-2 x \bar{s}+2 x c
\end{aligned}
$$

where $x u_{v}=x u-x u_{s}$ and $x d_{v}=x d-x d_{s}$ are the valence quark distributions of the proton. The average of both neutrino and anti-neutrino, $x \bar{F}_{3}$, represents the total valence quark distribution in the proton

$$
x \bar{F}_{3}=\frac{x F_{3}^{\nu N}+x F_{3}^{\bar{\nu} N}}{2}=x u_{v}+x d_{v}
$$

where we assume the symmetries $s=\bar{s}$ and $c=\bar{c}$. Under the same assumption, the difference of $x F_{3}^{\nu N}$ and $F_{3}^{\bar{\nu} N}, \Delta x F_{3}$ is given by

$$
\Delta x F_{3}=x F_{3}^{\nu N}-x F_{3}^{\bar{\nu} N}=2 x(s+\bar{s}-c-\bar{c})=4 x(s-c) .
$$

This quantity is sensitive to the strange and charm sea distributions in the proton.

Charged-lepton scattering, which is mediated by a virtual photon, is sensitive to quark charge, while neutrino scattering is sensitive to the helicity and flavor of the constituents. The electromagnetic charged-lepton structure functions, $2 x F_{1}^{l}$ and $F_{2}^{l}$, are given by

$$
\begin{aligned}
2 x F_{1}^{l} & =\sum_{i} e_{i}^{2}\left[x q_{i}^{l}+x \bar{q}_{i}^{l}\right] \\
F_{2}^{l} & =\sum_{i} e_{i}^{2}\left[x q_{i}^{l}+x \bar{q}_{i}^{l}+2 k_{i}^{l}(x)\right]
\end{aligned}
$$

where $e_{i}$ is the charge of the parton $i$. The parity-violating structure function, $x F_{3}$, which arises from the $\mathrm{V}$-A form of the weak interaction, is not present in charged-lepton scattering. 
Electromagnetic structure functions for the proton and neutron can be constructed using the quark densities and the quark charges

$$
\begin{aligned}
& 2 x F_{1}^{l p}=\left(\frac{1}{3}\right)^{2}[x d+x \bar{d}+x s+x \bar{s}]+\left(\frac{2}{3}\right)^{2}[x u+x \bar{u}+x c+x \bar{c}], \\
& 2 x F_{1}^{l n}=\left(\frac{1}{3}\right)^{2}[x u+x \bar{u}+x s+x \bar{s}]+\left(\frac{2}{3}\right)^{2}[x d+x \bar{d}+x c+x \bar{c}] .
\end{aligned}
$$

$2 x F_{1}^{l N}$ for an isoscalar target is

$$
2 x F_{1}^{l N}=\frac{2 x F_{1}^{l p}+2 x F_{1}^{l n}}{2}=\frac{5}{18}(x u+x \bar{u}+x d+x \bar{d})+\frac{1}{9}(x s+x \bar{s})+\frac{4}{9}(x c+x \bar{c}) .
$$

Assuming that the longitudinal structure function $R_{L}$ is the same for the electromagnetic neutral current and the weak charged current, we obtain $\frac{2 x F_{1}^{l N}}{2 x F_{1}^{\nu N}}=\frac{F_{2}^{l N}}{F_{2}^{\nu N}}$. Comparing $F_{2}^{l N}$ and $F_{2}^{\nu N}$ gives

$$
\frac{F_{2}^{l N}}{F_{2}^{\nu N}}=\frac{5}{18}\left(1-\frac{3}{5} \frac{x s+x \bar{s}-x c-x \bar{c}}{q+\bar{q}}\right) .
$$

where $q+\bar{q}=2 x F_{1}^{l N}$. This ratio is known as the " $5 / 18$ th" rule. It will be used to compare the structure functions from charge-lepton and neutrino experiments.

\subsection{QUANTUM CHROMODYNAMICS}

The QPM is valid in the infinite momentum frame in which the point-like constituents are onshell and quasi-free. This picture explains the scaling of the structure functions observed by early DIS experiments, but it doesn't explain the dynamics between the partons. A free quark has never been observed even though at high $Q^{2}$ quarks behave like free particles. A detailed study of the structure functions shows that the scaling behavior is only an approximation and that there are logarithmic scaling violations. In early DIS experiments (SLAC [8]) it was found that quarks and anti-quarks carry only half of the nucleon's momentum. The other half was assigned to the gluons which mediate the strong interaction and can produce sea quark-antiquark pairs. A new theory was needed to explain these phenomena. 
Quantum chromodynamics (QCD) is a non-Abelian gauge theory of the strong interaction [9]. It provides a consistent theory of the dynamics of quarks and gluons. There are several important consequences which arise from the non-Abelian structure of QCD. The gauge particles (gluons) carry color charge and can interact with each other. As a result, quarks are confined within hadrons because the self-interaction of the gluons anti-screens the color charge. The strong force between quarks increases with distance; at short distances (high $Q^{2}$ ) the quarks behave like free particles. This property is know as "asymptotic freedom".

Asymptotic freedom allows high-energy scattering to be calculated using a perturbative expansion. If the strong coupling constant, $\alpha_{S}$, is small we can expand in orders of $\alpha_{S}$. For example the Feynman diagrams shown in Figure 1.4 are the terms of order $\alpha_{S}^{1}$ in the expansion or "leading order" (LO) in QCD. Processes of order $\alpha_{S}^{2}$ are referred to as "next-toleading order" NLO in QCD, etc. As the strong coupling constant becomes larger we need to include higher order diagrams. Cross sections are calculated analytically using perturbative QCD (pQCD). Experimental measurements of physical quantities include all diagrams of all orders.

\subsubsection{Renormalization}

The inclusion of higher order diagrams results in ultraviolet divergences. These divergences are controlled by a renormalization procedure which depends on an arbitrary parameter $\mu^{2}$, where $\mu$ has the dimension of a mass. The coupling constant is defined to have a fixed finite value at some scale, $\mu^{2}$, and then the value of the coupling constant at any scale, $\alpha_{S}\left(Q^{2}\right)$, is expressed in terms of this fixed value. Physical quantities should be independent of the choice of $\mu^{2}$. The renormalization scale dependence of the effective QCD coupling constant, $\alpha_{s}$, us given by

$$
\mu \frac{d \alpha_{s}\left(\mu^{2}\right)}{d \mu}=-\frac{\beta_{0}}{2 \pi} \alpha_{S}^{2}\left(\mu^{2}\right)-\frac{\beta_{1}}{8 \pi^{2}} \alpha_{S}^{3}\left(\mu^{2}\right),
$$

where the coefficients $\beta_{0}$ and $\beta_{1}$ depend on the number of quark flavors $n_{f}$ and are given by

$$
\begin{aligned}
& \beta_{0}=11-2 n_{f} / 3 \\
& \beta_{1}=102-38 n_{f} / 3 .
\end{aligned}
$$


$q$
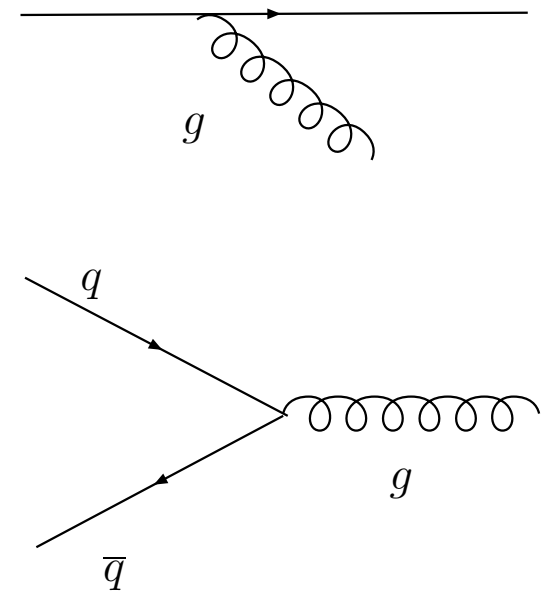

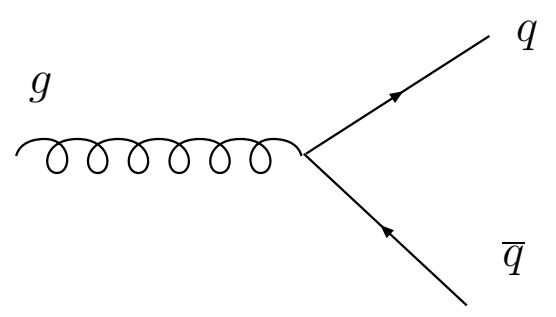

$g$

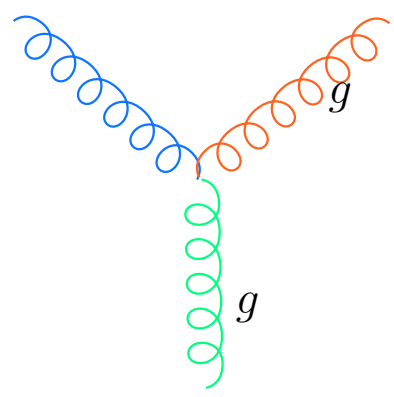

Figure 1.4: LO QCD diagrams.

The solution of this equation gives the scale dependence of the coupling constant. In solving Equation 1.42 a constant of integration is introduced, $\alpha_{S}\left(\mu_{0}^{2}\right)$, the value of the strong coupling constant at a reference scale $\mu=\mu_{0}$ (usually chosen to be $\mu=M_{Z}$ ). This is a fundamental constant of QCD which must be determined from experiment. A next to leading order (NLO) solution gives

$$
\alpha_{S}\left(Q^{2}\right)=\frac{4 \pi}{\beta_{0} \ln \left(Q^{2} / \Lambda^{2}\right)}\left[1-\frac{\beta_{1}}{\beta_{0}^{2}} \frac{\ln \left(\ln \left(Q^{2} / \Lambda^{2}\right)\right)}{\ln \left(Q^{2} / \Lambda^{2}\right)}\right]
$$

where $\Lambda$ is a dimensional parameter which must be determined experimentally. The measurement of $\Lambda$ is equivalent to measuring $\alpha\left(\mu_{0}^{2}\right)$. If $Q^{2}$ value is much larger than $\Lambda$ the effective coupling constant is small. In this region we have quasi-free partons and pQCD is applicable. 


\subsubsection{Factorization}

The factorization theorem of QCD [10] states that the structure functions can be factorized into two parts, a hard-scattering coefficient, $C_{a}^{l}\left(\frac{x}{y}, Q^{2}, \mu_{F}, \alpha_{S}(\mu)\right)$, and parton distribution functions, $q_{l}\left(y, \mu_{F}\right)$

$$
F_{a}\left(x, Q^{2}\right)=\sum_{l} \int_{x}^{1} \frac{d y}{y} q_{l}\left(y, \mu_{F}\right) C_{a}^{l}\left(\frac{x}{y}, Q^{2}, \mu_{F}, \alpha_{S}(\mu)\right),
$$

where the sum is carried over all partons. Here $y$ is the fraction of nucleon's momentum carried by the parton and $\mu_{F}$ is the factorization scale. The factorization scale sets an arbitrary separation between the processes at large distance scale and those at short distance scale. Below $\mu_{F}$, all long-range processes are contained in the parton distribution functions $q_{l}\left(y, \mu_{F}\right)$, while above $\mu_{F}$ the physics at short distance is given by the hard scattering coefficients, $C_{a}^{l}$.

The hard-scattering coefficients represent the probability for scattering off a parton of type $l$. They are completely calculable in pQCD and depend on the $Q^{2}$, the renormalization scale, and the factorization scale. In contrast, the parton distribution functions are universal and independent of the particular hard scattering process. They depend only on the type of parton and the factorization scale, $\mu_{F}$. The parton distribution functions include all infrared long distance contributions from phenomena occurring at low energy.

Structure functions are measured experimentally and hard-scattering coefficients are calculable (up to a given order) from Feynman diagrams. Therefore, using the factorization theorem, the parton distributions can be extracted from measured structure functions.

\subsubsection{Evolution}

Parton distribution functions are not calculable in pQCD. However, if the parton distributions are known at some scale, $Q^{2}, \mathrm{QCD}$ can predict their value at any $Q^{2}$. The evolution of the parton distributions with $Q^{2}$ are given by the Dokshitzer-Gribov-Lipatov-Altarelli-Parisi 
(DGLAP) equations [11]

$$
\begin{aligned}
\frac{d q^{N S}\left(x, Q^{2}\right)}{d \ln Q^{2}} & =\frac{\alpha_{S}\left(Q^{2}\right)}{2 \pi} \int_{x}^{1} \frac{d y}{y} q^{N S}\left(y, Q^{2}\right) P_{q q}\left(\frac{x}{y}\right), \\
\frac{d q^{S}\left(x, Q^{2}\right)}{d \ln Q^{2}} & =\frac{\alpha_{S}\left(Q^{2}\right)}{2 \pi} \int_{x}^{1} \frac{d y}{y}\left[q^{S}\left(y, Q^{2}\right) P_{q q}\left(\frac{x}{y}\right)+G\left(y, Q^{2}\right) P_{q g}\left(\frac{x}{y}\right)\right], \\
\frac{d G\left(x, Q^{2}\right)}{d \ln Q^{2}} & =\frac{\alpha_{S}\left(Q^{2}\right)}{2 \pi} \int_{x}^{1} \frac{d y}{y}\left[q^{S}\left(y, Q^{2}\right) P_{g q}\left(\frac{x}{y}\right)+G\left(y, Q^{2}\right) P_{g g}\left(\frac{x}{y}\right)\right],
\end{aligned}
$$

where the "splitting functions" $P_{i j}(x / y)$ represent the probability that parton $j$ with momentum $y$ will be resolved as parton $i$ with momentum $x<y$. G is the gluon distribution function, $q^{N S}=\sum_{i}\left(q_{i}-\bar{q}_{i}\right)$ is the non-singlet, and $q^{S}=\sum_{i}\left(q_{i}+\bar{q}_{i}\right)$ is the singlet quark distribution.

Using the factorization theorem 1.45 we obtain the evolution of the structure functions.

$$
\begin{aligned}
& \frac{F_{2}\left(x, Q^{2}\right)}{x}=\int_{x}^{1} d y\left[C_{2}^{q}\left(\frac{x}{y}, Q^{2}, \mu^{2}\right) q^{S}\left(x, \mu^{2}\right)+C_{2}^{g}\left(\frac{x}{y}, Q^{2}, \mu^{2}\right) G\left(x, \mu^{2}\right)\right], \\
& F_{3}\left(x, Q^{2}\right)=\int_{x}^{1} d y\left[C_{3}^{q}\left(\frac{x}{y}, Q^{2}, \mu^{2}\right) q^{N S}\left(x, \mu^{2}\right)\right],
\end{aligned}
$$

where $C_{i}$ are the hard scattering coefficients. These evolution equations yield logarithmic scaling violations of the structure functions.

At leading order the quark hard-scattering coefficients $C^{q}$ are proportional to $\delta(1-x / y)$, while the coefficient for the gluon term $C^{g}$ vanishes. Therefore, at leading order

$$
\begin{aligned}
F_{2}\left(x, Q^{2}\right) & =x q^{S}\left(x, Q^{2}, \mu^{2}\right), \\
x F_{3}\left(x, Q^{2}\right) & =x q^{N S}\left(x, Q^{2}, \mu^{2}\right) .
\end{aligned}
$$

The scale dependence at leading order appears only in the parton distribution functions as a result of the quark splitting functions and is usually chosen to be $\mu^{2}=Q^{2}$.

Using the factorization theorem we can also calculate the QCD prediction for the longitudinal structure function $R_{L}=\sigma_{L} / \sigma_{T}$. In the simple QPM (Section 1.3) $R_{L}$ is zero. In leading order QCD $R_{L}=0$. However, in NLO QCD processes like quark-gluon bremsstrahlung and 
$q \bar{q}$ pair production give the quarks a transverse momentum $p_{T}$ which results in a non-zero $R_{L}$. For example a NLO QCD calculation [12] gives

$$
R_{L}^{N L O, Q C D}\left(x, Q^{2}\right)=\frac{\frac{\alpha_{s}}{\pi} \int_{x}^{1} \frac{d y}{y}\left[\sum_{i=q, \bar{q}} q_{i}(x) \sigma_{L}(x / y)+\sum_{i=q} G(x) \sigma_{L}(x / y)\right]}{\sum_{i=q, \bar{q}} q_{i}(x)+\frac{\alpha_{s}}{\pi} \int_{x}^{1} \frac{d y}{y}\left[\sum_{i=q, \bar{q}} q_{i}(x) \sigma_{L}(x / y)+\sum_{i=q} G(x) \sigma_{L}(x / y)\right]}
$$

which depends on the longitudinal cross section. $R_{L}$ can be good probe of the gluon distribution if the structure functions are well known.

\subsubsection{Non-Perturbative QCD Effects}

Non-perturbative QCD effects become important at low $Q^{2}$, where the resolving power of the virtual boson is not large enough to probe a single parton. In this region, partons can not be considered massless non-interacting particles. This can result in, for example, diquark scattering. In addition, the partons "effective mass" must be considered, leading to higher order corrections (target mass).

1.4.4.1 Higher Twist Effects At low $Q^{2}$ lepton-nucleon scattering can involve multiple partons shown on Figure 1.5. Diagrams for multiple parton scattering can not be calculated a priori with the means of pQCD to all orders. Compared to the leading twist diagrams (pQCD) the higher twist diagrams are suppressed by powers of $1 / Q^{2}$, so they are important at low $Q^{2}$. This is also the region where the strong coupling constant is large and pQCD is invalid.

1.4.4.2 Target Mass Effects In the original derivation of the differential cross section (Section 1.2.2) the masses of the quarks and the nucleon are neglected. However, at high $x$ and low $Q^{2}$ the target mass becomes important. In the Georgi-Politzer calculation [13] the fractional momentum of the quark is rescaled to account for the nucleon mass $M$

$$
x \rightarrow \xi=\frac{2 x}{(1+k)},
$$




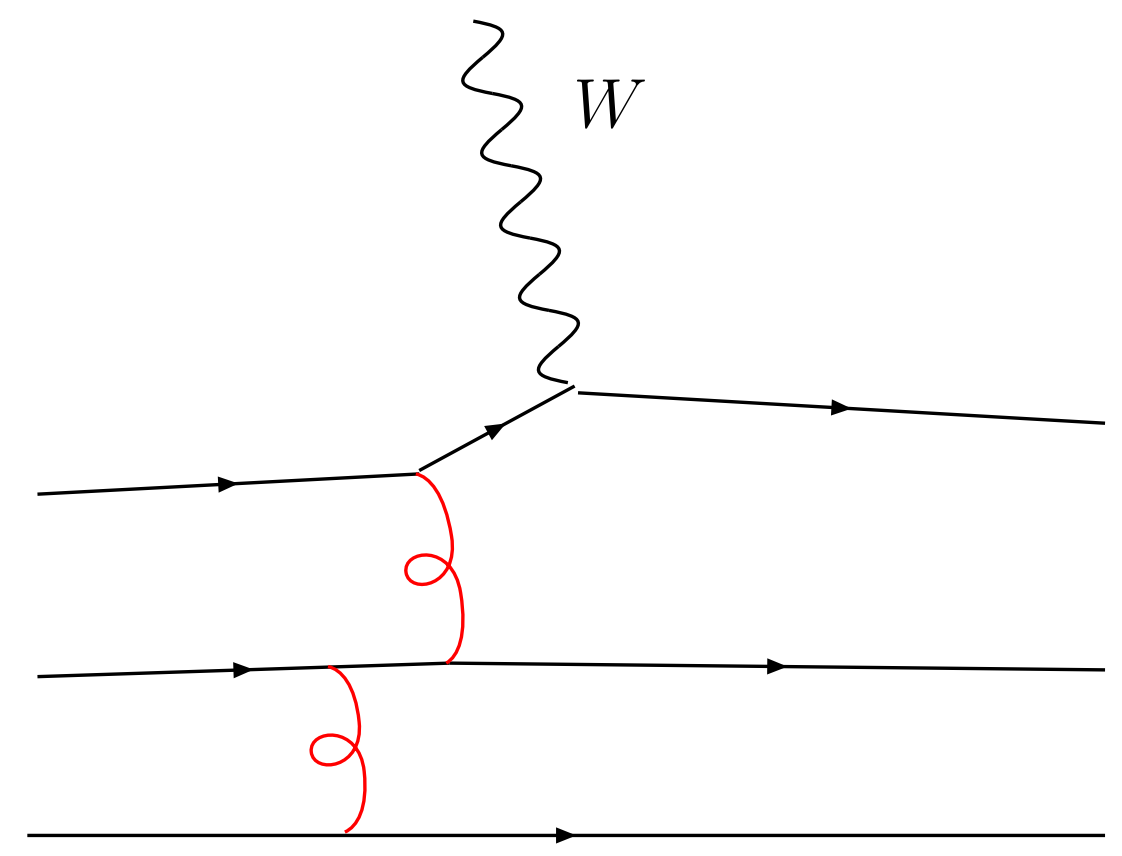

Figure 1.5: Feynman diagram for scattering from multiple partons.

where $k=\left(1+4 M^{2} x^{2} / Q^{2}\right)^{1 / 2}$. Target mass terms are incorporated in the structure functions using

$$
\begin{aligned}
F_{2}^{T M} & =\frac{x^{2}}{\xi^{2}} \frac{F_{2}^{Q C D}\left(\xi, Q^{2}\right)}{k^{3}}+\frac{6 M^{2}}{Q^{2}} \frac{x^{3}}{k^{4}} I_{1}+\frac{12 M^{4}}{Q^{4}} \frac{x^{4}}{k^{5}} I_{2}, \\
x F_{3}^{T M} & =\frac{x^{2}}{k^{2}} \frac{x F_{3}^{Q C D}\left(\xi, Q^{2}\right)}{\xi^{2}}+\frac{2 M^{2}}{Q^{2}} \frac{x^{3}}{k^{3}} I_{3},
\end{aligned}
$$

where $I_{i}$ are the integrals

$$
\begin{aligned}
& I_{1}=\int_{\xi}^{1} d u \frac{F_{2}^{Q C D}\left(u, Q^{2}\right)}{u^{2}} \\
& I_{2}=\int_{\xi}^{1} d u \int_{u}^{1} d \nu \frac{F_{2}^{Q C D}\left(\nu, Q^{2}\right)}{\nu^{2}}, \\
& I_{3}=\int_{\xi}^{1} d u \frac{x F_{3}^{Q C D}\left(u, Q^{2}\right)}{u^{2}} .
\end{aligned}
$$

The target mass effect is often referred to as "kinematic higher twist" because it involves powers of $1 / Q^{2}$. 


\subsection{DETECTOR AND BEAMLINE}

To perform a neutrino experiment we need a high intensity neutrino beam and a large area target with as many nucleons as possible to increase the probability of neutrino interaction in our detector. These requirements are a consequence of the fact that neutrinos interact weakly.

The NuTeV beamline was designed to produce a high flux of neutrinos and anti-neutrinos using separate high purity beams. The $\mathrm{NuTeV}$ detector is a massive, coarse-grained detector. This design is typical of neutrino detectors used to collect large samples of neutrino interactions. It consists of two parts; a target calorimeter where the neutrino interaction takes place, and muon spectrometer where the momentum of the muon produced in the interaction is measured. This chapter describes in detail both the NuTeV beamline and detector.

\section{$2.1 \quad$ NUTEV BEAMLINE}

\subsubsection{The Tevatron}

The Tevatron at Fermilab accelerates protons in several stages (Figure 2.1). Hydrogen ions $\left(H^{-}\right)$are accelerated to $750 \mathrm{KeV}$ with a five-stage Cockroft-Walton generator. Then a linear accelerator (Linac) accelerates them to $400 \mathrm{MeV}$ in two stages. The first stage operates at $201 \mathrm{MHz}$, and the second more effective stage operates at $805 \mathrm{MHz}$. The pulsed beam of $\mathrm{H}^{-}$ is then injected into booster. On the way to the booster the $H^{-}$pass through a carbon foil, which strips off the electrons and produces proton $p^{+}$beam. The booster is a synchrotron $140 \mathrm{~m}$ in diameter operating at $15 \mathrm{~Hz}$. It accelerates the protons to $8 \mathrm{GeV}$ energy over $0.033 \mathrm{~s}$. 


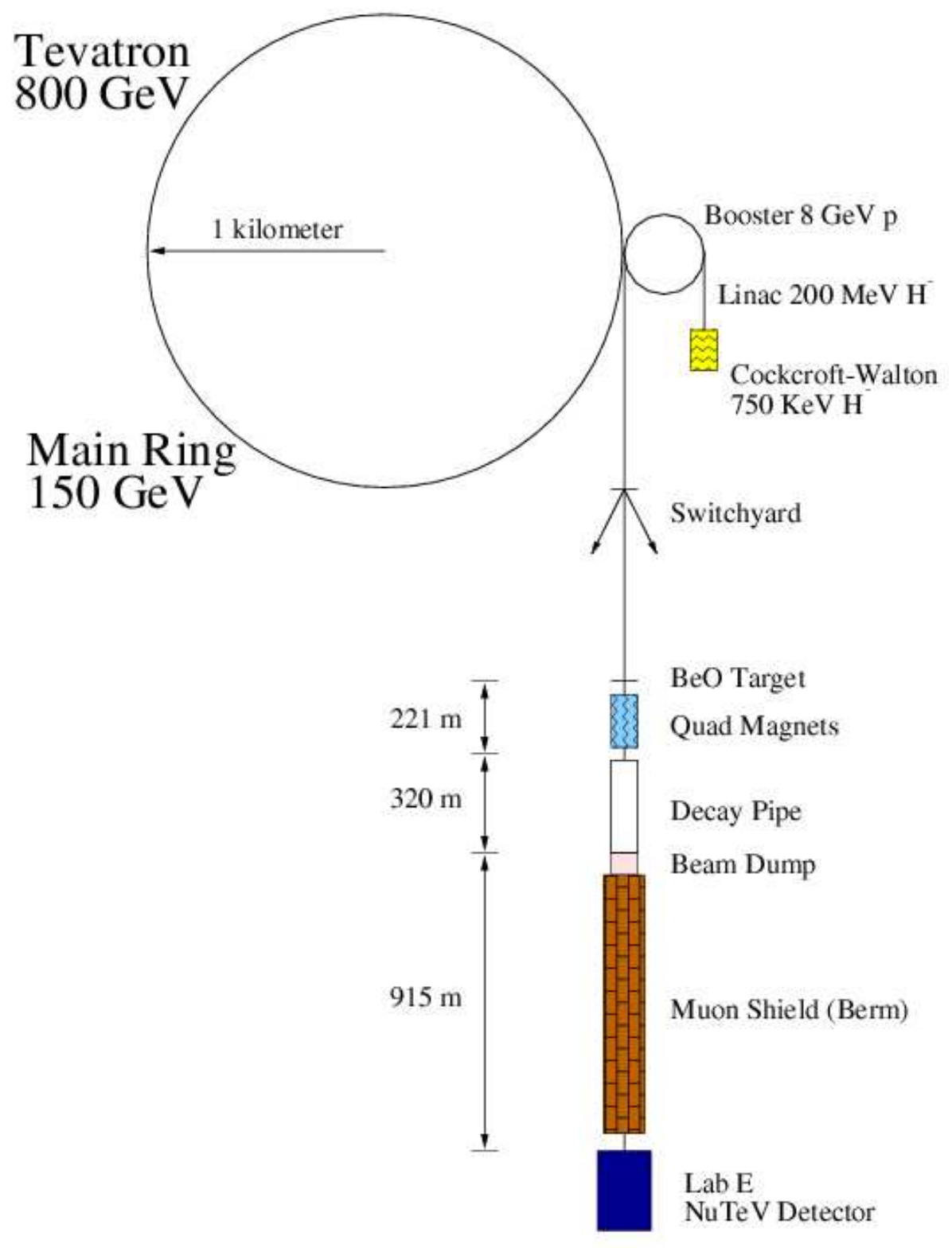

Figure 2.1: A schematic drawing of the $\mathrm{NuTeV}$ beamline layout at Fermilab. 


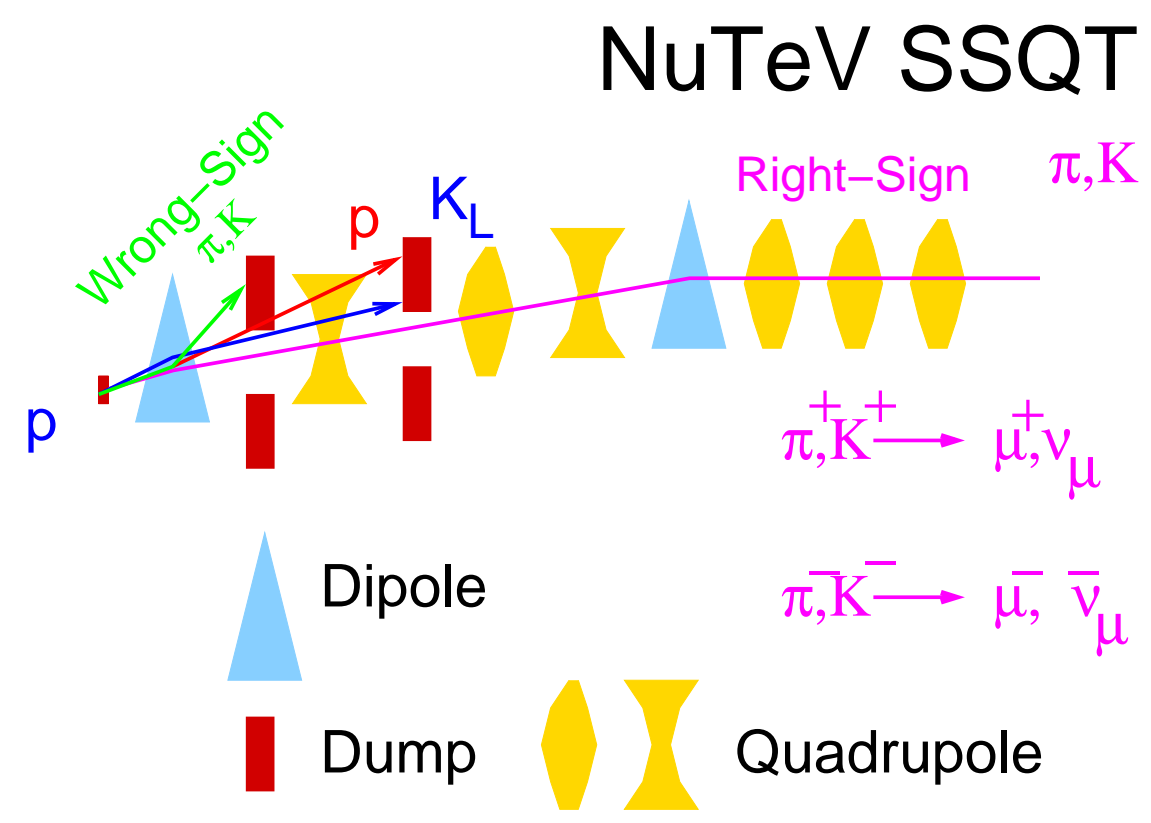

Figure 2.2: Edge view of the SSQT.

The beam is then sent to the Main Ring, $2 \mathrm{~km}$ diameter proton synchrotron. The Main Ring uses dipoles and quadrupoles to accelerate protons to $400 \mathrm{GeV}$. Once the proton beam reaches $150 \mathrm{GeV}$ it is injected into the Tevatron. The Tevatron is a superconducting synchrotron which shares the same tunnel with the Main Ring. The magnets of the Tevatron are made of superconductive material cooled to $4.6 \mathrm{~K}$ with liquid helium. In the Tevatron the protons are accelerated to $800 \mathrm{GeV}$ and then are sent to the switchyard. The switchyard distributes the beam to several beamlines, including NuTeV's neutrino beamline.

\subsubsection{Neutrino Beamline}

Protons arrive from the Tevatron at a 7.8 mrad angle (with respect to the $\mathrm{z}$ axis of $\mathrm{NuTeV}$ detector) with $800 \mathrm{GeV}$ and hit a low-Z target. The target is a $33 \mathrm{~cm}$ long $\mathrm{BeO}$ (Beryllium Oxide) rod, situated $1450 \mathrm{~m}$ upstream from the $\mathrm{NuTeV}$ detector. The length of the BeO target corresponds to one interaction length. The neutrino beam results from the decays of mesons, mostly $\pi$ 's and K's, produced in the primary target. 
Secondary particles produced in the primary target enter the Sign Selected Quadrupole Train (SSQT) [14] shown in Figure 2.2. A series of strong dipole magnets bend the "rightsign" mesons towards the $\mathrm{NuTeV}$ detector, while the opposite sign "wrong-sign" mesons are bend to point away and hit the first dump. Upstream neutral particles go forward into the second upstream dump. The protons which did not interact are stopped in the second upstream dump. The "right-sign" $\pi, K$ are allowed to decay in-flight in a $300 m$ decay region which begins immediately downstream of the last focusing magnet. Muons and other decay products are stopped in a $900 \mathrm{~m}$ berm of earth and steel which follows the decay region. The thick shielding region allows only neutrinos to go through and enter NuTeV detector situated at Lab E.

As a result of this charge selection in the SSQT, NuTeV has high purity separate neutrino $\nu_{\mu}$ and anti-neutrino $\bar{\nu}_{\mu}$ beams. The wrong-sign contamination in "neutrino mode" is $0.03 \%$ anti-neutrinos, and in "anti-neutrino mode" $0.4 \%$ are neutrinos. There is also a small component of electron neutrinos $\nu_{e}$ and $\bar{\nu}_{e}$, which are a result of the decay $K^{ \pm} \rightarrow \pi^{0} e^{ \pm} \nu_{e}\left(\overline{\nu_{e}}\right)$. These amount to about $1.6 \%$ of the beam in neutrino mode and $1.7 \%$ in anti-neutrino mode. Another source of $\nu_{e}$ and $\overline{\nu_{e}}$ in high energy neutrino beamlines is $K^{0}$, which are produced at poorly known rate in the proton target. As shown in Figure 2.2, because of the vertical bends in the SSQT the $K_{L}$ point away from the direction of the $\mathrm{NuTeV}$ detector and therefore the SSQT almost completely eliminates this source of uncertainty. This feature is important for the NuTeV's precision measurement of $\sin ^{2} \theta_{\mathrm{W}}$.

Figure 2.3 shows a prediction for the neutrino and anti-neutrino flux energy spectrum obtained from beam Monte Carlo.

\subsubsection{Beam Timing Structure}

The Tevatron operating in fixed-target mode has a $60.1 \mathrm{~s}$ cycle. The neutrinos arrive in five "pings" $0.5 m s$ each $5 m s$ apart once per cycle. The calibration beam spill arrives approximately $1.4 s$ after the neutrino "pings" and has an $18 s$ duration each cycle. The beam timing structure is shown in Figure 2.4. Due to this timing structure the events are classified as follows: 

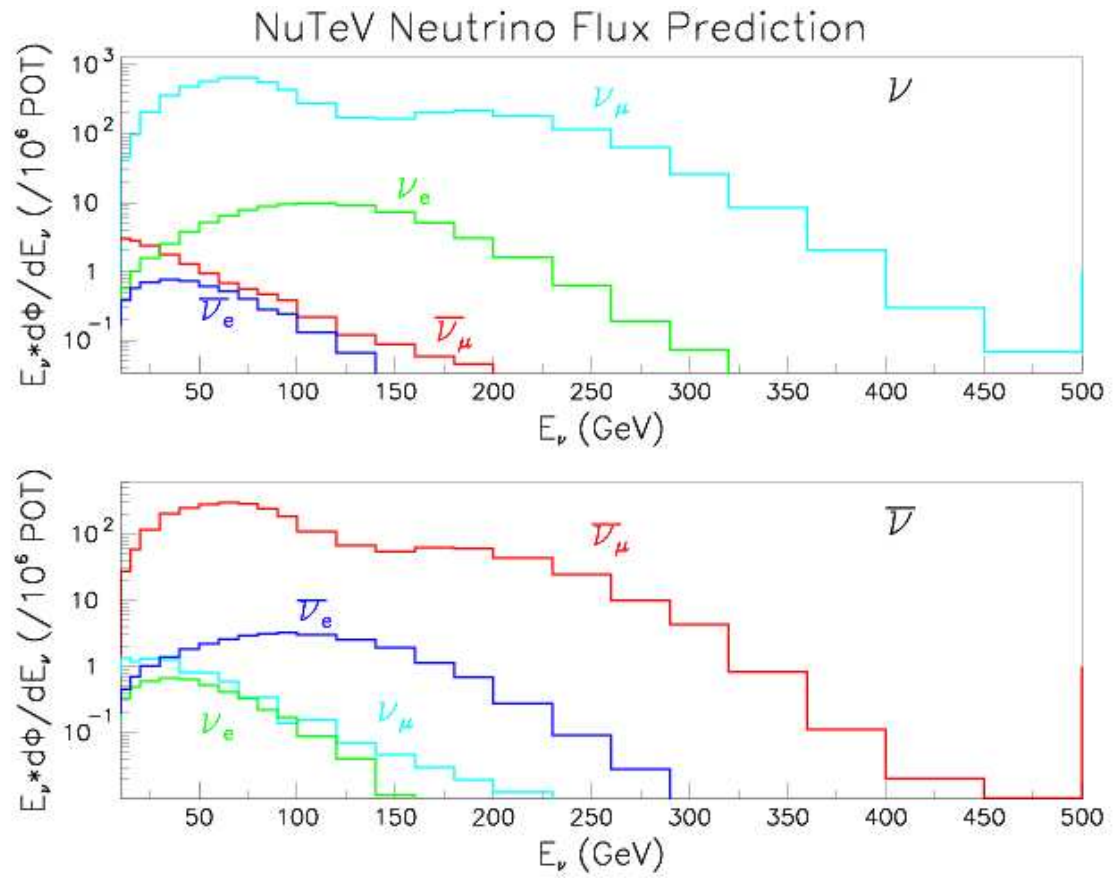

Figure 2.3: Flux prediction from beam Monte Carlo for neutrino (top) and anti-neutrino (bottom) mode.

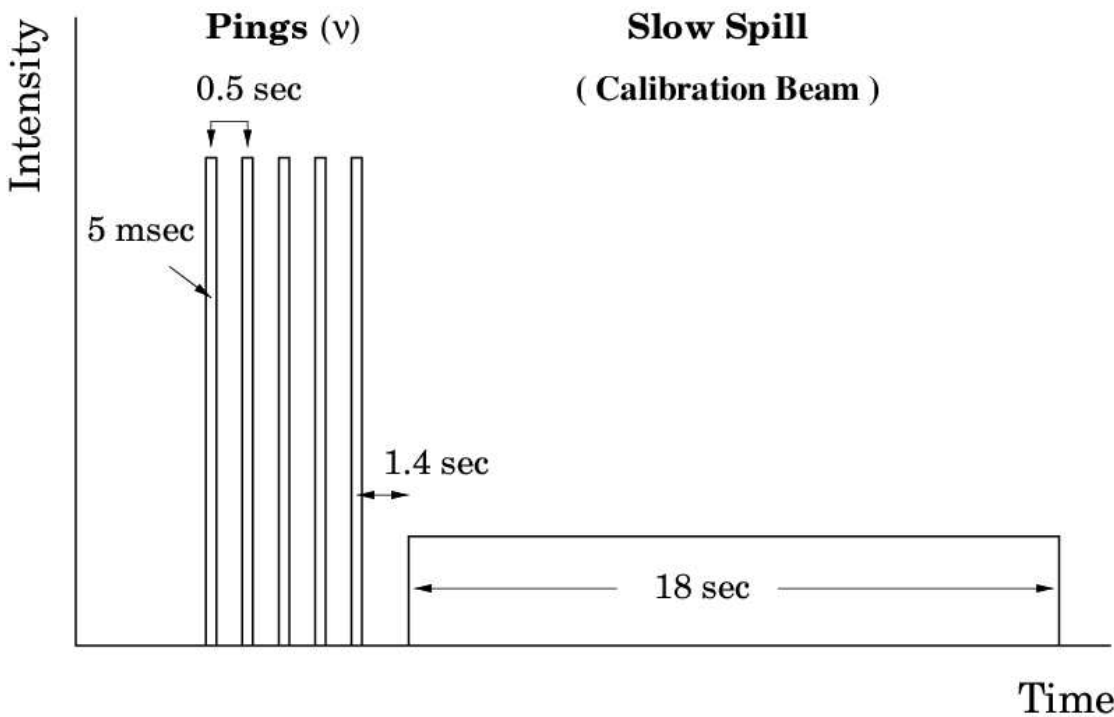

Figure 2.4: Beam timing structure. 


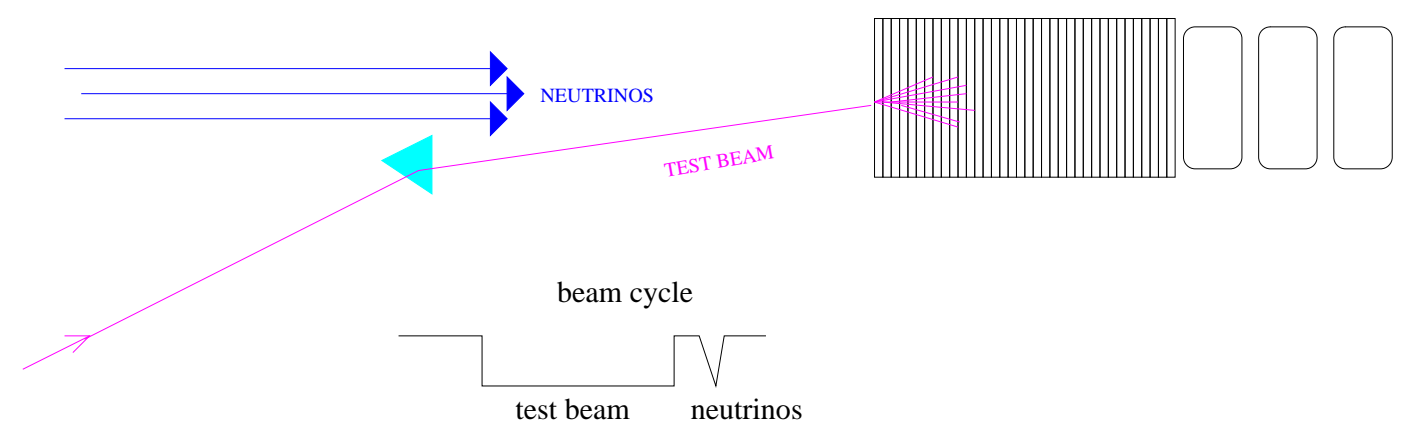

Figure 2.5: A schematic drawing of the test beam layout.

- Fast Gate (gates 1-5): Pings last $0.5 m s$ each and deliver $1-2 \times 10^{12}$ protons on target (POT) per ping. Five pings in total form the fast spill. This is the neutrino beam gate.

- Slow Gate (gate 6): Starts $1.4 s$ after the last ping of fast spill and stays on for $18 s$. This is the calibration beam gate (also called test beam gate). The calibration of the detector was carried out using this test beam which allowed "continuous" calibration over the entire run approximately once per minute.

- Cosmic Ray Gate (gate 7): Cosmic ray triggers are accepted during this gate which is on once per cycle for $5 s$ when the beam is off.

\subsubsection{Calibration Beam}

In order to calibrate the detector response and measure the absolute muon and hadron energy scales NuTeV designed the "test beam" beamline [15] shown on Figure 2.5. The test beam was incident at a 43 mrad angle on the $\mathrm{NuTeV}$ detector and delivered high purity beams of hadrons, muons and electrons with a wide energy range (4.8 to $190 \mathrm{GeV}$ ). It allowed $\mathrm{NuTeV}$ to calibrate the detector continuously within each cycle during the slow gate.

The test beam consisted of a low mass spectrometer instrumented with drift chambers for tracking, dipole bend magnets and a Čerenkov detector along with series of transition radiation detectors (TRDs) for particle identification. The particle ID was used to identify pions, kaons, electrons, and anti-protons. When particle ID was not needed this section was 


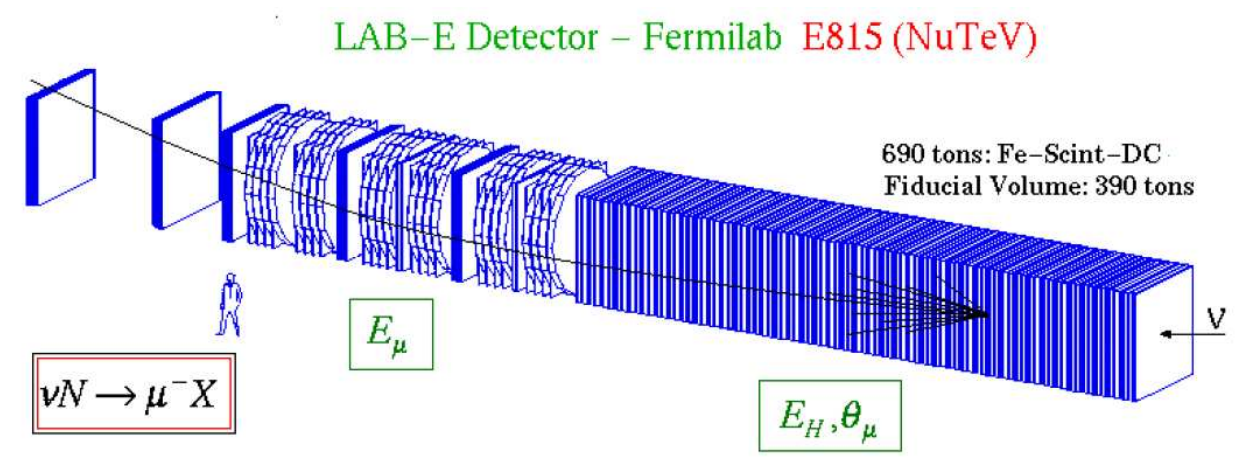

Figure 2.6: The $\mathrm{NuTeV}$ detector.

rolled out and replaced by vacuum pipe. The last dipole in the spectrometer magnet string could be rotated to allow the beam to be directed to any point on the front face of the $\mathrm{NuTeV}$ detector.

The spectrometer is designed to measure the absolute momentum of the test beam particles to better than $0.3 \%$ on an event-by-event basis. This is accomplished by two means. First, precisely calibrated dipole magnets are used, with $\int B d \ell$ known to better than $0.1 \%$ in the region traversed by the beam. Also, the bend angle is measured to better than $0.1 \%$ using drift chambers positioned over the $500 \mathrm{~m}$ beamline, which provides a long lever arm. This long length of the spectrometer chamber spacing allows us to tolerate a relative chamber alignment uncertainty of $1 \mathrm{~mm}$. The event-by-event resolution was $0.3 \%$, which was dominated by multiple Coulomb scattering (MCS).

\subsection{NUTEV DETECTOR}

The NuTeV detector [15] shown in Figure 2.6 consists of two parts (described below in more detail). The upstream part is the target calorimeter, where the neutrino interaction takes place. The target calorimeter measures the energy of the hadron shower, $E_{H A D}$, produced in the interaction, and the angle of the muon track, $\theta_{\mu}$, at the vertex. The downstream 


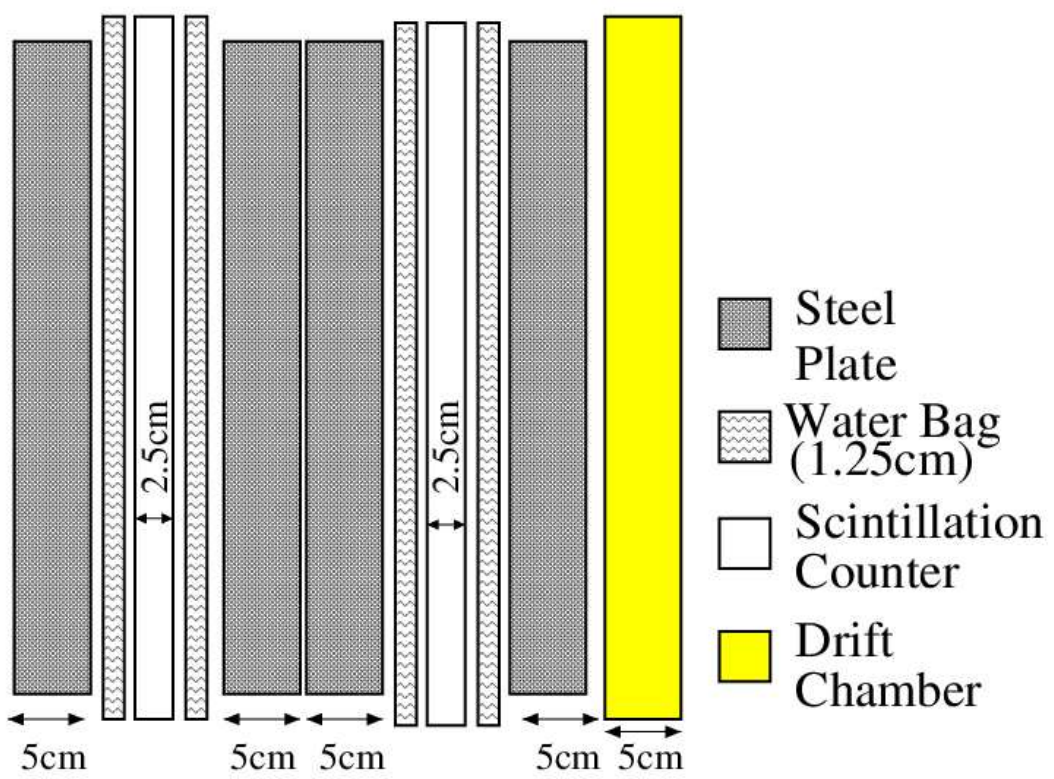

Figure 2.7: A schematic drawing of one target unit.

part is the muon spectrometer (also called the toroid), which measures the momentum of the muon, $p_{\mu}$, produced in the neutrino interaction. The kinematics of the interaction are reconstructed from three variables $E_{H A D}, \theta_{\mu}$, and $p_{\mu}$.

\subsubsection{Target Calorimeter}

NuTeV's target calorimeter has an active area of $3 m \times 3 m$ and a total length along the beam direction of $17.7 \mathrm{~m}$. It is constructed of 168 steel plates of $5.1 \mathrm{~cm}$ thickness, 84 liquid scintillation counters (one every 2 steel plates), and 42 drift chambers (one every 4 steel plates). The arrangement of one calorimeter unit is shown in Figure 2.7. One unit consists of 4 steel plates, 2 scintillation counters and one drift chamber. The length, radiation length $X_{0}$ and interaction length $\lambda_{I}$ of each of the components in a single calorimeter unit are given in Table 2.1. The target calorimeter is made up of 42 calorimeter units arranged in 6 carts. A neutrino interacts in a steel plate (high Z). The produced particles deposit signals in scintillation counters and drift chambers (low Z). Each active component of the detector 


\begin{tabular}{|l|c|c|c|}
\hline Component & Length $(\mathrm{cm})$ & Radiation Length $\left(X_{0}\right)$ & Interaction Length $\left(\lambda_{I}\right)$ \\
\hline 4 steel plates & 20.7 & 11.75 & 1.24 \\
2 scintillation counters & 13 & 0.51 & 0.16 \\
1 drift chamber & 3.7 & 0.17 & 0.03 \\
\hline \hline Total & 37.4 & 12.43 & 1.43 \\
\hline
\end{tabular}

Table 2.1: Length, radiation length and interaction length for the components of one calorimeter unit. from reference [15].

is described below.

2.2.1.1 Scintillator Counters The energy deposited by charged particles is sampled by liquid scintillation counters. There are 84 scintillation counters numbered from 84 (most upstream counter) to 1 (most downstream counter). They are instrumented as a scintillatorphotomultiplier tube (PMT) system. One scintillation counter is $2.5 \mathrm{~cm}$ thick and contains roughly 65 gallons of Bicron 517L liquid scintillator oil. The sides of each scintillator are supported by $3 \mathrm{~mm}$ thick vertical Lucite ribs which are spaced approximately $2.5-5 \mathrm{~cm}$ apart and run along the length of the counter. Since the ribs do not scintillate the ribs of consecutive counters are purposely staggered to avoid creating of dead zones. In addition, to balance the pressure of the oil in the scintillation counter there are two plastic water-filled bags on each side of the counter. The counter is viewed by PMTs mounted at the four corners of the counter. Figure 2.8 shows front and side views of a $\mathrm{NuTeV}$ scintillation counter.

When a charged particle passes through the scintillator, it excites the primary fluor, which emits ultraviolet light. This ultraviolet light excites a secondary fluor which emits light in the visible spectrum (blue). The emitted blue light dominates the light output because it has longer attenuation length. In order to improve the response of the PMTs eight half-inch thick wavelength-shifter bars surround the counter. The wavelength-shifter bars contain a third fluor which shifts the blue light to a longer wavelength (green). The green light is then collected via total internal reflection to the phototubes in each of the 
corners of the counter.

NuTeV uses 10-stage Hamamatsu R2154 phototubes with green-extended photocathodes, which are maintained at approximately $1400 \mathrm{~V}$. The photocathode has a $20 \%$ probability of converting a photon to an electron via the photoelectric effect. The single electron is multiplied by on the order of $10^{6}$ by a series of dynodes. The signal from a single muon traversing the center of the counter is about 30 photoelectrons. The signal of a muon traversing near the edges of the counter is higher.

2.2.1.2 Drift Chambers Drift chambers are used to track muons in the target calorimeter and toroid. There are 42 three-wire per cell drift chambers within the target and 19 single-wire per cell chambers within the toroid. Each chamber is constructed from $3 \mathrm{~m} \times$ 3m Hexcel-covered aluminum walls. Figure 2.9 shows the geometry of one drift chamber. It consists of two orthogonally oriented planes (X-view and Y-view). Each plane is divided into 24 cells of 5 inches wide. The upper and lower surfaces of each cell are covered with copper-clad G10 panels which are milled to form a set of 19 cathode strips per cell. The strip voltage is held at $-4500 \mathrm{~V}$. It is supplied by I-beams at the edge of each cell. In order to maintain a uniform electric field across the drift space the voltage is distributed decrementally to each strip via a resistor card (voltage divider). A charged particle passing through the chamber ionizes the gas along its path. The electrons drift towards the anode wire. The electric field near the wire is very high and it accelerates the electrons, liberated by ionization to amplify the signal (avalanche). If the drift velocity of the electrons is constant, the drift time is a linear function of the distance to the anode wire. A constant drift velocity of $v_{D}=52.4 \mu \mathrm{m} / \mathrm{ns}$ is achieved by using an equal parts argon-ethane gas mixture for which the electron drift velocity is independent of the electric field when the field is large. It takes the electron $1.2 \mu$ s to cross half a drift cell.

The particle position in one dimension was determined from the difference between the arrival time of the drift chamber pulse, $T^{0}$, and the event time, $T$, (set by the trigger). This gives much better resolution than the size of the chamber. The X-Y position of the particle 


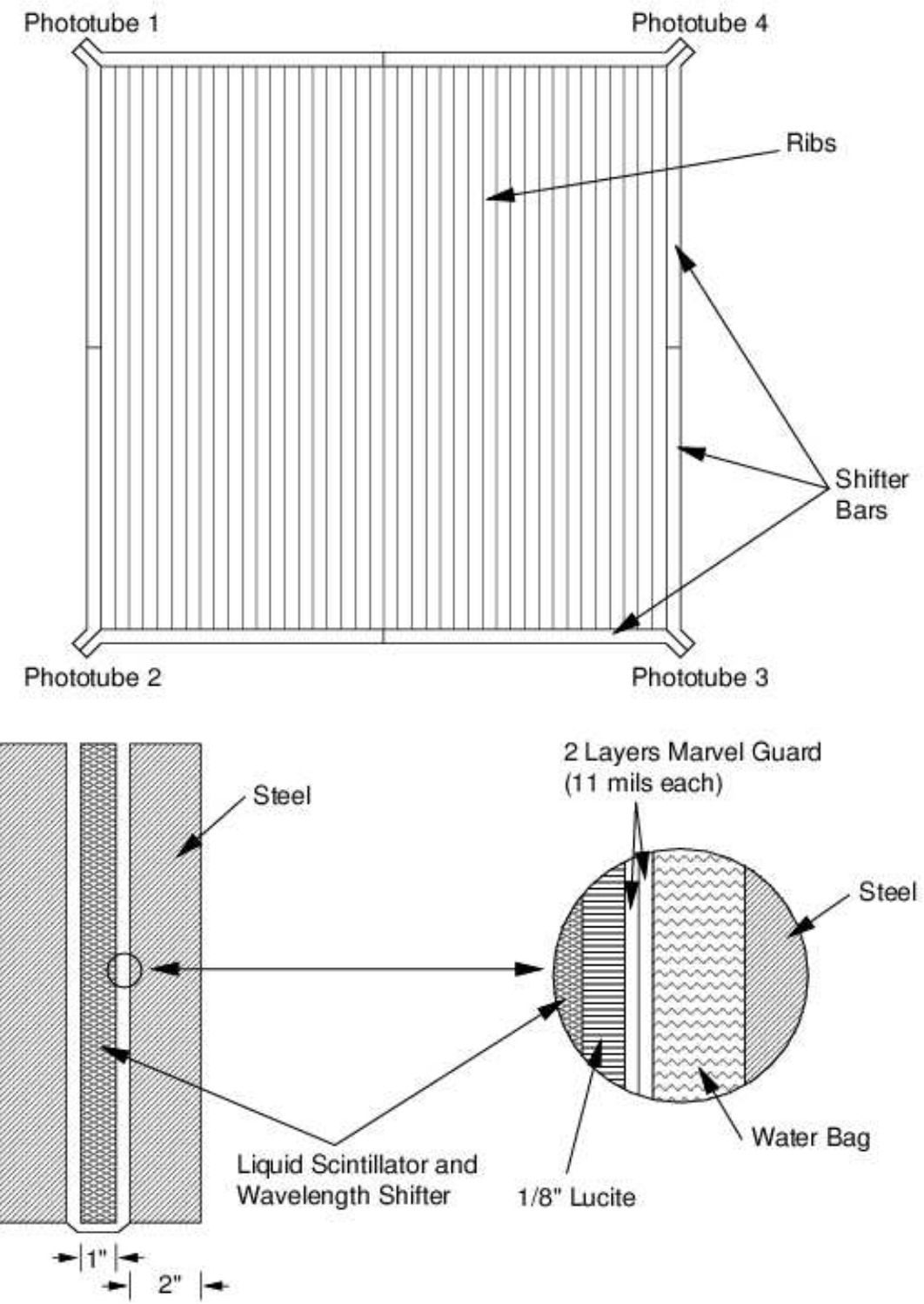

Figure 2.8: Front view (top) and cross section (bottom) of a scintillation counter. 


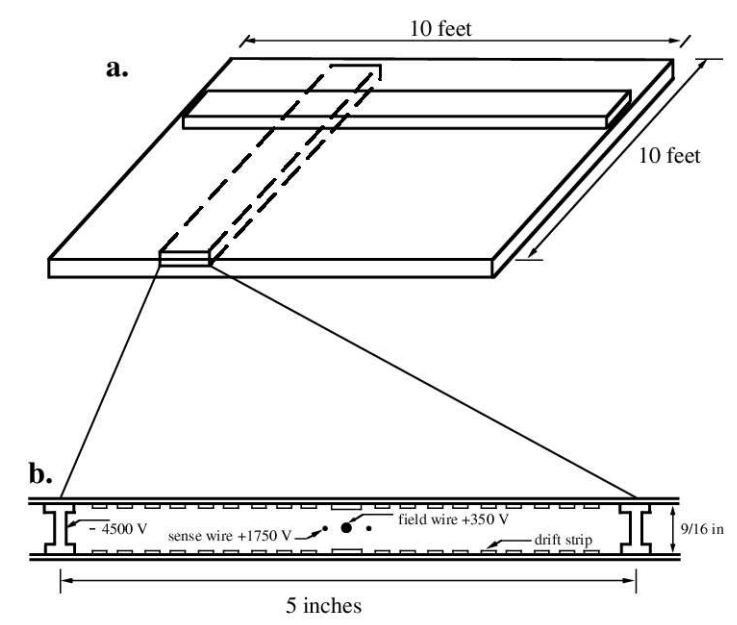

Figure 2.9: Top view of a drift chamber (top) and cross section of three-wire DC (bottom).

in the chamber is determined by:

$$
\begin{aligned}
& x=x^{0}+v_{D}\left(T-T_{x}^{0}\right), \\
& y=y^{0}+v_{D}\left(T-T_{y}^{0}\right),
\end{aligned}
$$

where $x^{0}$ and $y^{0}$ are the locations of the sense wires.

\subsubsection{Muon Spectrometer}

The muon spectrometer consist of three toroidal magnets and five sets of drift chambers. Each magnet consists of eight steel washers with $3.6 \mathrm{~m}$ outer diameter and $24 \mathrm{~cm}$ diameter inner hole. There are four copper coils with 1200A current, which magnetize each toroid. The magnetic field was $1.5 \mathrm{~T}$ near the outer edge and $1.9 \mathrm{~T}$ near the hole, and is confined to the volume of the toroids. The polarity of the magnetic field, controlled by the direction of the current in the coils, is set that during neutrino mode negatively charged particles are focused (bent towards the center), and during anti-neutrino mode positively charged particles are focused. Figure 2.10 shows a schematic drawing of the cross section of the toroid. 


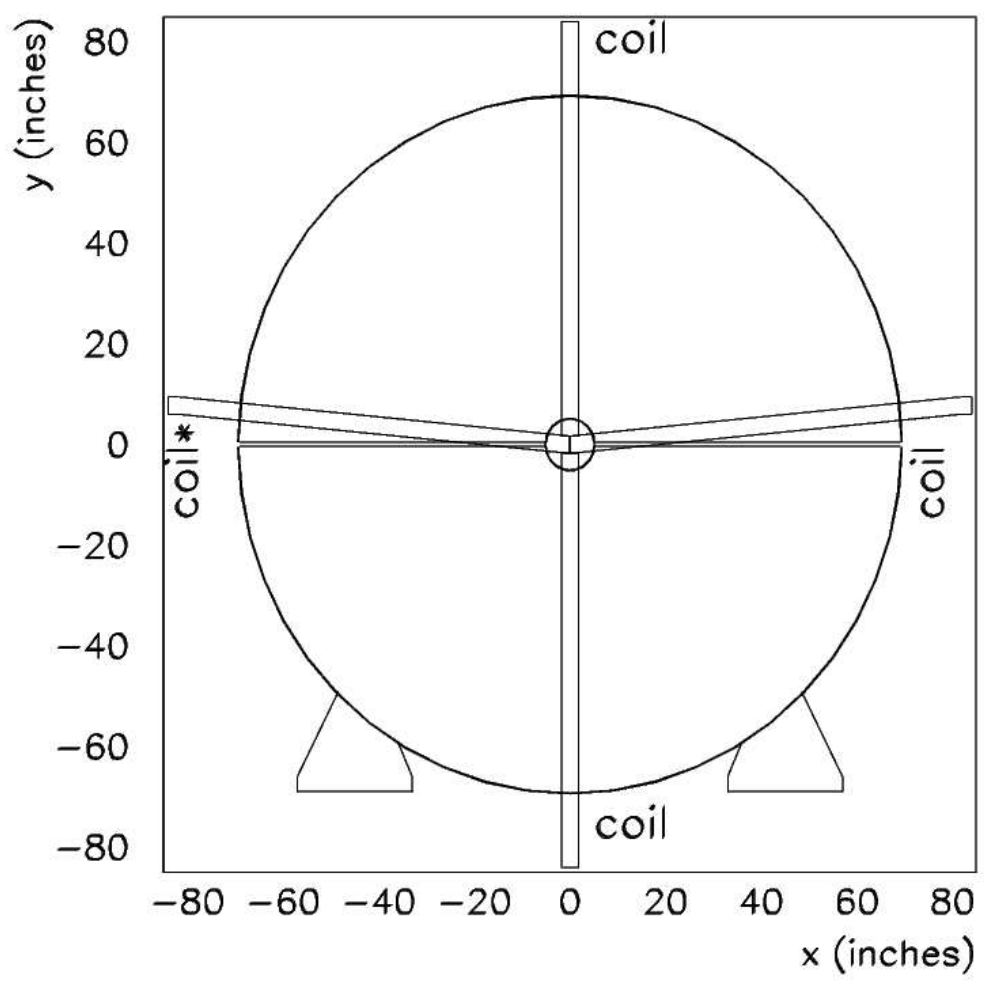

Figure 2.10: Schematic drawing of the cross section view of the toroid. The coil marked with '*' was shunted to ground early in the run and disconnected. 

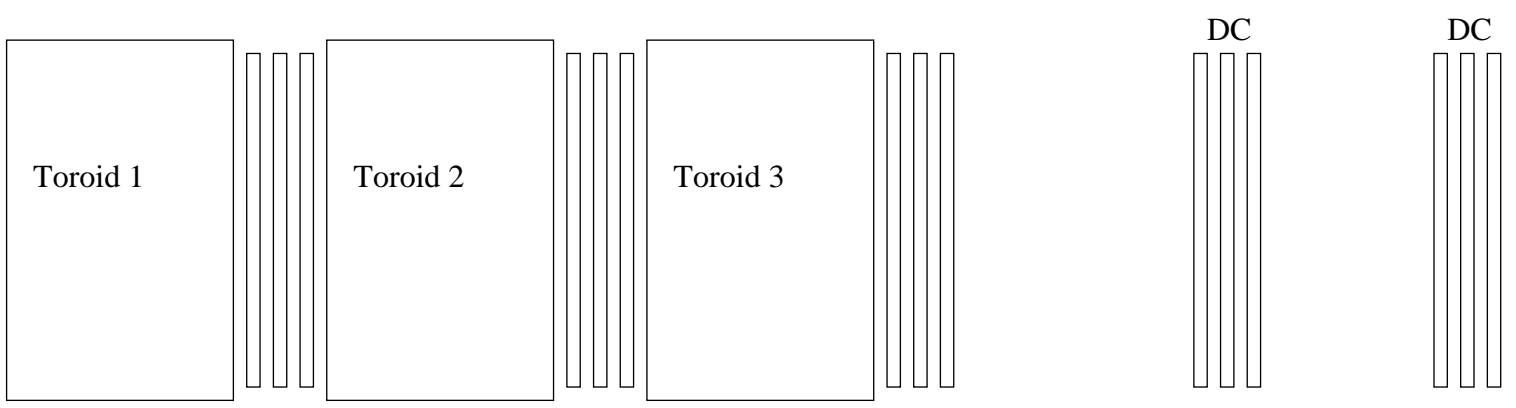

Figure 2.11: Schematic drawing of the side view of the toroid. Drift chamber layout is shown.

A muon traversing the whole length of the toroid will receive on average a total of $2.4 \mathrm{GeV} / \mathrm{c}$ additional transverse momentum. Three sets of five drift chambers, situated in the space (gap) right after each toroid, provide information about the X-Y position of the muon, which allows the reconstruction of the muon track. Two sets of 3 chambers (blue cart), situated on $2.4 \mathrm{~m}$ and $6.2 \mathrm{~m}$ downstream of the last chamber in the toroid, provide an additional lever arm for measurement of high momentum muons. Figure 2.11 shows a schematic drawing of the drift chamber layout throughout the toroid.

In the beginning of the run one of the coils (shown with asterisk on Figure 2.10) of the first toroid shorted to ground and was disconnected. In order to compensate for the missing coil, the current in the remaining three coils was increased to produce the same magnitude of the magnetic field. Small asymmetry in the magnetic field was present, due to the missing coil.

$\mathrm{NuTeV}$ used magnetic field map which is obtained from a finite-element analysis simulation program called ANSYS [17]. The detailed geometry of the toroid (including the missing coil) and the $\mathrm{BH}$ hysteresis curve corresponding to the toroid's steel were inputs to the ANSYS simulation. Figure 2.12 shows the simulated magnetic field after calibration (see Section 3.3.3). 


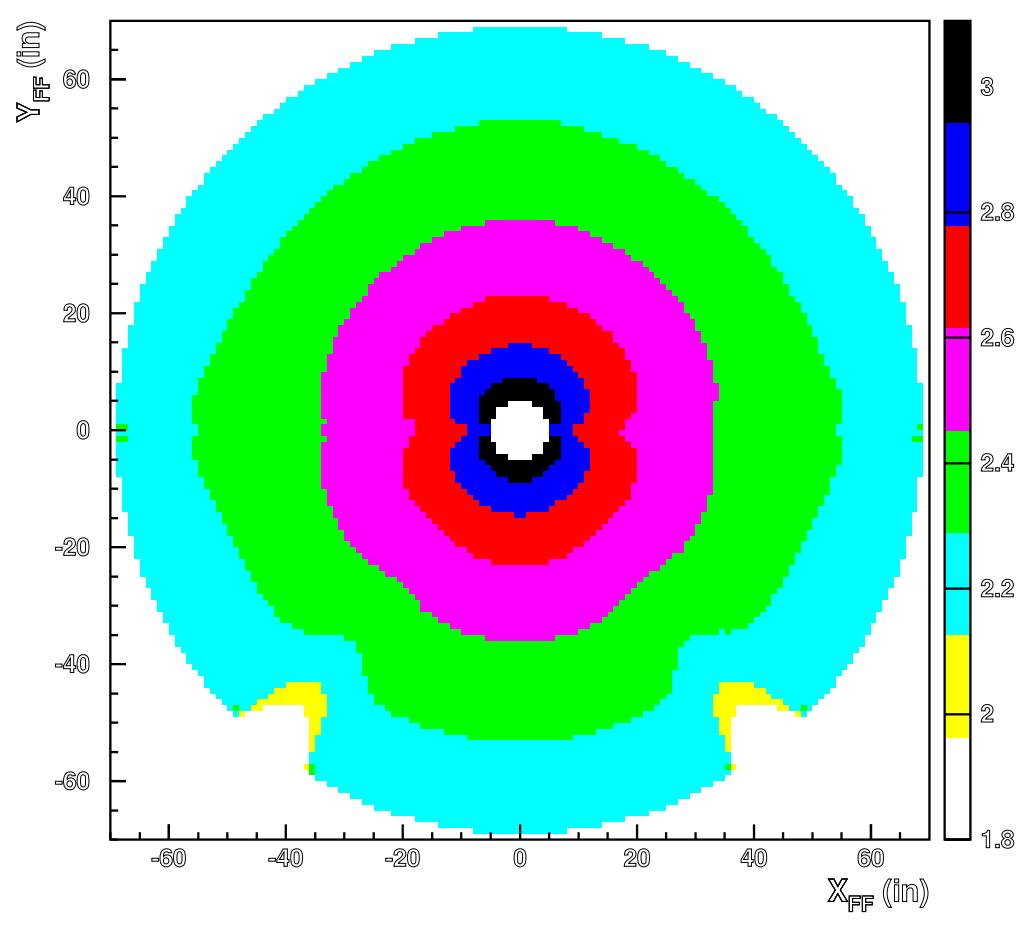

Figure 2.12: $B_{\phi}$ component of the magnetic field in units of $\mathrm{GeV} / \mathrm{c}$ after calibration. 


\section{$2.3 \quad$ READOUT ELECTRONICS AND EVENT TRIGGERING}

\subsubsection{Drift Chamber Signal Read-out}

The raw signals from the drift chambers are pre-amplified and sent as an ECL logic pulse to be digitized by the time-to-digital converter (TDC). The TDC digitized the time in 4ns bins and it was able to buffer 512-bit words, which gives a live time window of $2 \mu \mathrm{s}$. The maximum drift time is $1.2 \mu \mathrm{s}$, hence the TDC is able to buffer the whole drift range of the drift chamber during the live time window.

\subsubsection{PMT Signal Read-out}

The raw signal for the energy deposited in a scintillation counter from the phototubes is integrated and converted by 11-bit ADC (analog-to-digital converter) to "ADC counts". There is a wide range of energies registered by a scintillation counter. A muon, for example, will typically deposit $0.2 \mathrm{GeV}$ in a counter, while a hadron shower can deposit $100 \mathrm{GeV}$ in a single counter. This wide range of energies requires three separate ADC gain read-out streams to convert the raw signal to ADC count. They are HIGH gain, LOW gain and SUPERLOW gain.

- LOW : For each counter the raw signal from the 4 PMT's is digitized separately. Hence there are four LOWS per counter. A muon traversing the center of the counter will produce approximately 2 ADC counts in a LOW channel.

- COMBINATION LOW : The combined signal of all 4 phototubes is digitized. A typical muon passing through the center of a counter will produce approximately 8 ADC counts in a COMBINATION LOW channel.

- HIGH : The combined signal of all 4 phototubes (COMBINATION LOW) is amplified 10 times and digitized. In this case a typical muon will produce 80 ADC counts in a HIGH channel.

- SUPERLOW : The combined signal of 8 phototubes from 8 different counters (each separated by 10 counters) is formed. This signal is attenuated by 6 or 12 depending on the fan-out. A typical muon will produce 0.2 counts in this channel. 
The HIGHs are typically used to measure the muon energy. The LOWs are used to measure the energy of hadron showers, which usually saturate the HIGHs. For a counter in which one of the LOWs is saturated, SUPERLOWS are used. COMBINATION LOWs are used to form event triggers. A schematic drawing of the $\mathrm{NuTeV}$ calorimeter read-out electronics is shown in Figure 2.13. The test beam is used to find the relation between the ADC counts and the corresponding energy in $\mathrm{GeV}$ for each type of particle.

The signals from the phototubes are also discriminated and the timing from each counter is recorded in TDCs. Logical signals, "bits", which indicate a minimum signal in a given counter, were constructed with different discrimination logic as follows:

- Sbit (single particle indicator): The combination of the 4 PMT signals of a single counter is multiplied by $100(\mathrm{HIGH} \times 10)$ and discriminated with $150 \mathrm{mV}$ threshold. This is designed to identify at least one muon passing through the counter.

- Tbit (more than one particle): The combination of the 4 PMT signals of a single counter is multiplied by $100(\mathrm{HIGH} \times 10)$ and discriminated with $450 \mathrm{mV}$ threshold. This is designed to distinguish between a shower and a single particle.

- Nbit (shower indicator): The linear sum of the phototube signal from every combination of 8 consecutive counters is channeled without amplification through a discriminator with a $55 \mathrm{mV}$ threshold $(5 \mathrm{GeV})$.

\subsubsection{Event Triggers}

Event triggers are designed to select the events which will be digitized and recorded. Data triggers preselect certain event signatures and reduce the amount of background and noise. $\mathrm{NuTeV}$ constructed twelve data triggers:

- Trigger 1 - Charge Current: Designed to select events for which a muon originated in the target and the passed through part of the toroid. One of the following two requirements are needed to register the trigger: 1) Hits in 2 of the last 4 counters in the target and hits in both toroid gaps; 2) Hits in 2 of the last 4 counters in the target or hits in 2 of counters 9-12 and hits in the first toroid gap only. In both cases a "no veto" 


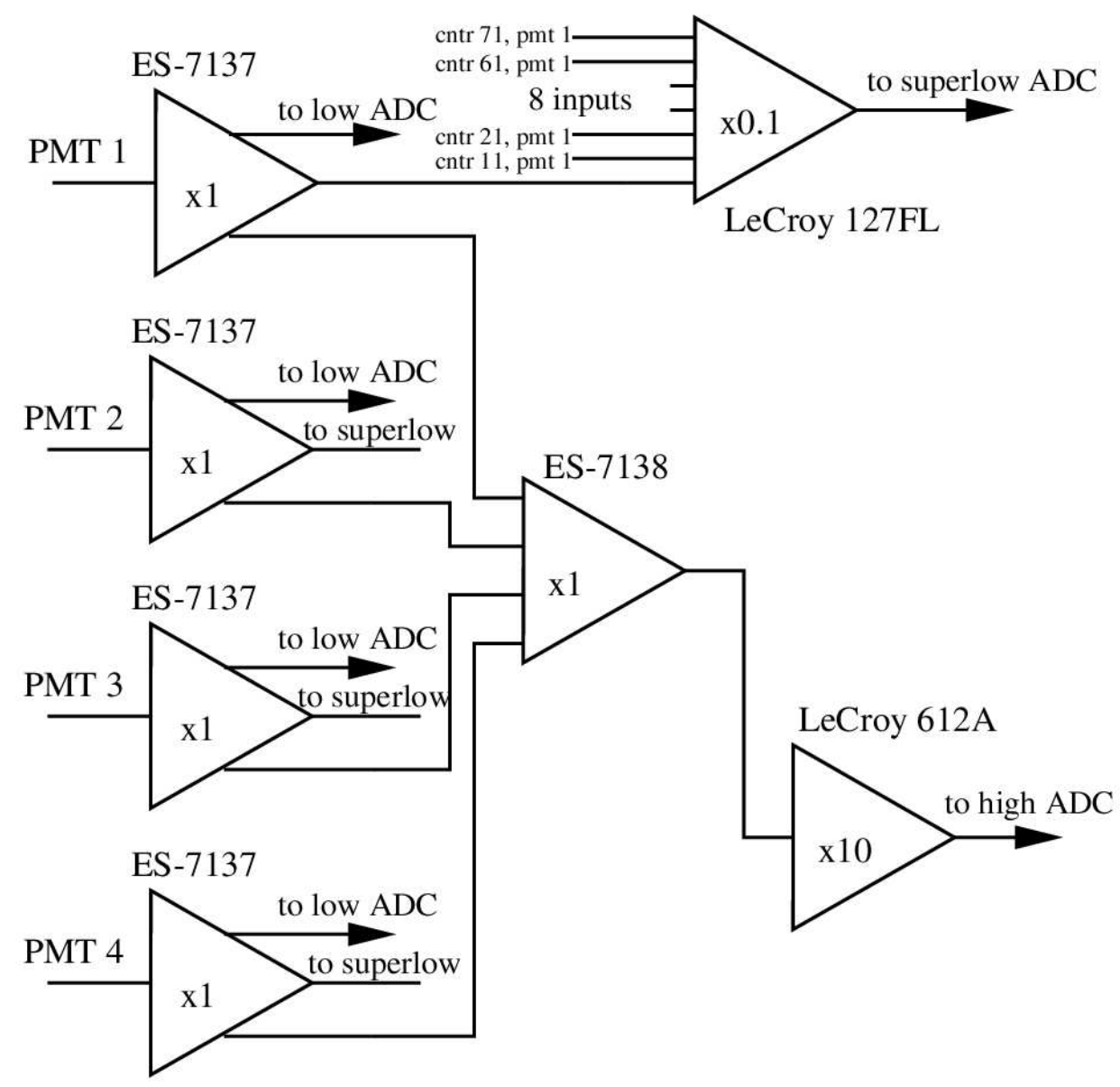

Figure 2.13: A schematic drawing of the PMT read-out electronics. 
signal was required in the upstream most counter. This trigger selects events for the charged-current cross section analysis.

- Trigger 2 - Neutral Current: Designed to select a shower with minimum penetration. This trigger requires the energy in 8 consecutive counters to be at least $5 \mathrm{GeV}$. A 'No veto' signal was required as well.

- Trigger 3 - Penetrating Muon: Required 16 counters in the target to be above Sbit threshold, at least $4 \mathrm{GeV}$ deposited energy in any 8 consecutive counters and a 'no veto' signal.

- Trigger 4 - Redundant Charge Current: Used to check trigger 1, but uses different hardware and has slightly stricter geometric requirements.

- Trigger 5 - Calibration Beam: Required a charged particle to go through test beam spectrometer. There was no requirement for energy deposited in the calorimeter.

- Trigger 6 - Straight Through Muon: Selected muons produced upstream of the detector. Veto signal was required and at least one Sbit in each of the target carts, and hits throughout the toroid in the same quadrant. This trigger was used for drift chamber alignment and counter calibration.

- Trigger 8 - Cosmic Ray: selected off-spill cosmic ray muons. Required 40 semiconsecutive counters and hits in the first toroid gap.

- Trigger 9 - NHL: selected candidates for the neutral heavy lepton (NHL) analysis.

- Trigger 10-12 - Pedestal Triggers: Allowed extraction of information from the detector when there is minimal activity. Pedestals are taken systematically during each run, when no other triggers have fired.

Table 2.2 shows the number of neutrino gate events for each trigger. 


\begin{tabular}{|c|c|c|c|}
\hline Trigger & Description & $\nu$-mode & $\bar{\nu}$-mode \\
\hline 1 & Charge Current & 2612830 & 908161 \\
2 & Neutral Current & 5445024 & 2804981 \\
3 & Penetrating Muon & 4306217 & 3065412 \\
6 & Straight Through Muon & 1712591 & 735222 \\
9 & Neutral Heavy Lepton & 1334675 & 1616637 \\
10 & In-Spill Pedestal & 189885 & 183794 \\
11 & Toroid Pedestal & 785040 & 153461 \\
\hline
\end{tabular}

Table 2.2: Number of neutrino gate events for different triggers recorded during $\mathrm{NuTeV}$ run. 


\subsection{DETECTOR CALIBRATION AND DATA ANALYSIS}

To construct the DIS kinematic variables three physics quantities must be measured: the energy of the hadron shower, $E_{H A D}$, the angle of the muon at the vertex, $\theta_{\mu}$, and the muon energy, $E_{\mu}$. To accomplish this, the electronic signals from the detector have to be converted into energies and track vectors. This chapter describes the event reconstruction and defines the data samples.

\subsection{HADRON ENERGY RECONSTRUCTION AND CALIBRATION}

The energy of the hadron shower deposited in the calorimeter is sampled by the energy deposited in the scintillation counters. The pulse heights $(\mathrm{PH})$ from each phototube is stored as an ADC count. A shower deposits only a small fraction of its energy in the scintillation counters. Most of the energy is deposited in the steel plates. For example, a $100 \mathrm{GeV}$ shower will traverse about 10 counters and it will deposit only about $3 \%$ of its energy in the scintillation counters. The data from the calibration beam and the straight-through muon sample are used to calibrate the response of the counters. This is needed to convert the ADC count signal from the LOWs and the HIGHs into energy of a shower in GeV. The energy, $E_{\text {cntr }}(i)$, in counter ' $i$ ' is measured from the pulse height, $P H(i)$, which is registered in either LOW or HIGH channels. For this counter

$$
E_{c n t r}(i)=\frac{h(i) \cdot G(i, t) \cdot P H(i)}{M\left(i, V_{x}, V_{y}, t\right)} .
$$

where $G(i, t), M\left(i, V_{x}, V_{y}, t\right)$ and $h(i)$ are the counter gain, the counter map correction and the relative hadron gain function, respectively, which will be discussed below. $P H(i)$ is the 
number of ADC counts from the LOW channel, (if the signal is in the linear region, which corresponds to more than 35 counts in the LOW channel). If the number of ADC count in the LOW channel is less than 35 the HIGH channel is used. If one of the LOW channels is saturated, i.e. more than 1900 ADC counts, the SUPERLOW channel is used. The pedestals, which register the detectors response when no trigger has fired during the neutrino run, are subtracted from $P H(i)$.

The counter gain, $G(i, t)$, is used to convert the ADC count to minimum ionizing particles (MIPs). The mean energy deposited by a muon with an energy of $77 \mathrm{GeV}$ is defined as 1 MIP. To calibrate each counter's gain, trigger 6 muons are used. These muons are produced in neutrino interactions in the berm upstream of the $\mathrm{NuTeV}$ detector. They illuminate the detector in a similar way as neutrino interactions in the detector and coincide in time with the neutrino gate, which makes them an excellent calibration tool. A muon deposits on average 30 photoelectrons per counter, which corresponds to about 80 ADC counts in a HIGH channel.

Counter response is different for each counter due to variations in the geometry and furthermore, it varies over time. The relative hadron gain function, $h(i)$, accounts for these variations and non-uniformities.

The response of the counters also depends on the location of the traversing muon. This is due to the geometry of light collection. A muon, traversing near the center of the counter, will produce fewer ADC counts on average than a muon traversing near the corner(close to one of the PMT's). The pulse heights are corrected with a counter map correction, $M(i, x, y, t)$, which is the ratio of the counter response at coordinates $(x, y)$ to the counter response at the center $(0,0)$. The straight through muon sample is used to obtain this correction. The counter map correction has typical values in the range between 0.5 and 3.0. For each counter, the pulse heights are corrected with $M\left(i, V_{x}, V_{y}, t\right)$, where $V_{x}, V_{y}$ is the transverse vertex for the event. If the value is outside the range $0.1<M\left(i, V_{x}, V_{y}, t\right)<5.0$ no correction is applied. Figure 3.1 shows the average response of a counter to muons as a function of the position in the counter.

The conversion factor $C_{\pi}$ converts pulse height, which is in MIPs, to GeV. $C_{\pi}$ is obtained from hadron test beam data by fitting the means of the event-by-event distributions of the 


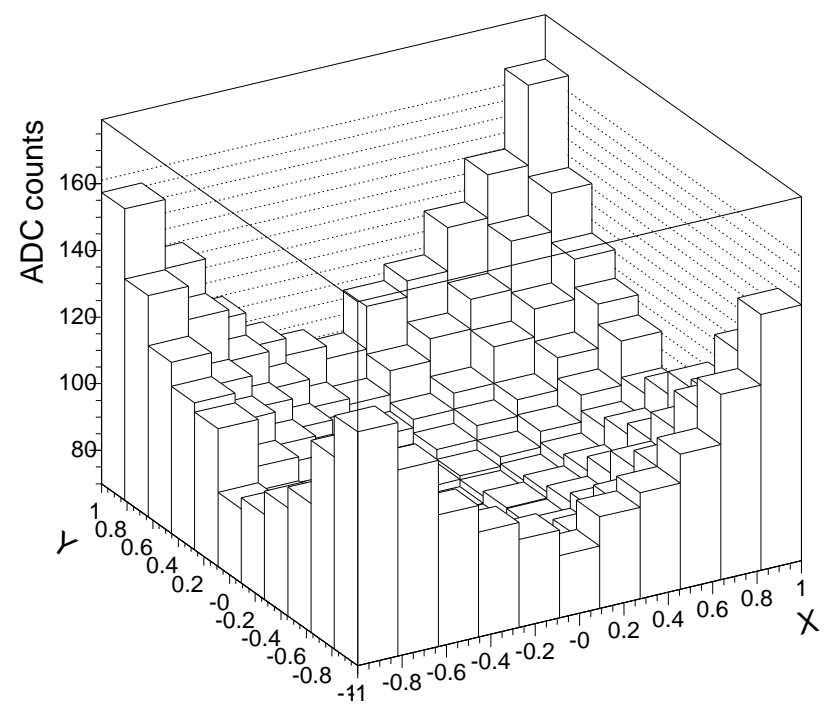

Figure 3.1: Average counter response to muons as function of the position.

ratio of the test beam momentum to the energy measured by the calorimeter [15]. The measured value is $C_{\pi}=0.212 \mathrm{GeV} / \mathrm{MIP}$. This has been adjusted so that the mean ratio of hadron response from the calorimeter compared to with test beam momentum at $75 \mathrm{GeV}$ is $1.000 \pm 0.001 . C_{\pi}$ is discussed further below.

The detector response is a non-linear function with energy. This small non-linearity is due to the difference in detector response for hadronic and electromagnetic showers. Figure 3.2 shows a $3 \%$ non-linearity over the range from 5 to $190 \mathrm{GeV}$ in hadron energy. $\pi^{0}$ produced in hadron showers decay quickly to two photons initiating an electromagnetic cascade. Electromagnetic cascades have a much shorter characteristic length than hadron induced cascades. The calibration constant for "real" showers, containing both types of cascades, is

$$
C_{\pi}=e \cdot f_{\pi^{0}}(E)+h \cdot\left[1-f_{\pi^{0}}(E)\right],
$$

where $e$ is the conversion factor for a pure electromagnetic shower, $h$ is the corresponding factor for pure hadron showers, and $f_{\pi^{0}}(E)$ is the fraction of $\pi^{0}$ 's produced in the shower. 


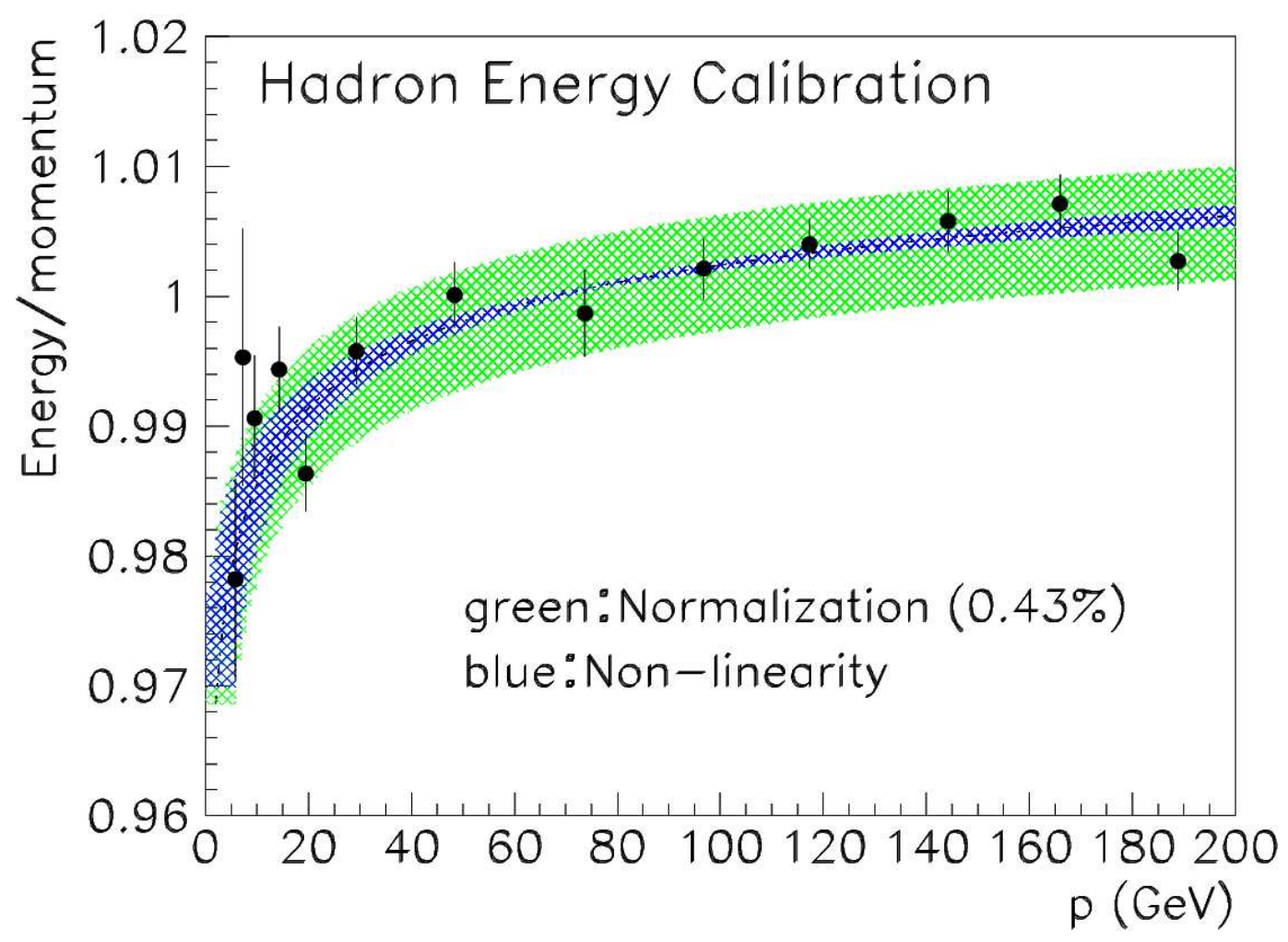

Figure 3.2: The calibration fit to test beam hadrons, which determines the non-linearity. The point at $75 \mathrm{GeV}$ is adjusted to $1.000 \pm 0.001$. 


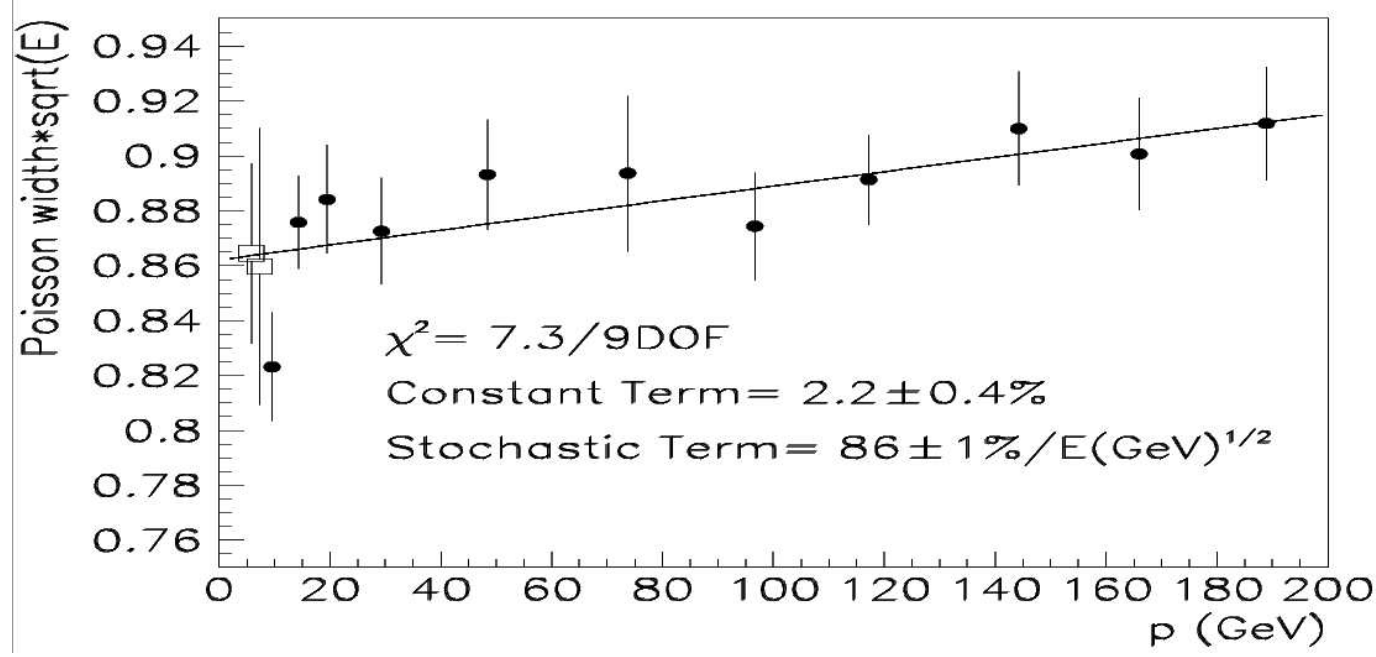

Figure 3.3: The width of the Poisson-like response function as a function of energy, obtained from a fit to the detector response for test beam hadrons.

Using Groom's parameterization [16], $f_{\pi^{0}}(E)=1-\left(\frac{E_{H A D}}{0.96 G e V}\right)^{-0.184}$, the fit to hadron energy response gives $\frac{e}{h}=1.079 \pm 0.011$. The normalization uncertainty determines the uncertainty in $C_{\pi}$, which is $0.43 \%$. This gives the absolute hadronic energy scale uncertainty.

The uncertainty in the measurement of a single hadron shower is given by the hadronic resolution function of the calorimeter. The detector response to a monoenergetic beam of hadrons is a Poisson-like function, because the reconstructed energy is proportional to the number of particles produced in the shower. Statistical fluctuations in the production of electromagnetic particles makes this distribution Poisson-like and at high energies, Gaussian. The test beam data provides a measure of the resolution function $\sigma(E) / E$, which is parameterized as

$$
\frac{\sigma(E)}{E}=A \oplus \frac{B}{\sqrt{E}} \oplus \frac{C}{E}
$$

where $A$ is the constant term, $B$ is the stochastic term from the sampling of the shower, and $C$ is the term associated with the noise due to pedestal subtraction. Data showed no evidence for noise, hence the term $C$ is removed from the fit. Figure 3.3 shows the fit for the 
width parameter of the resolution function as function of energy. The width of the Poisson distribution is found to be

$$
\frac{\sigma(E)}{E}=\frac{0.86 \pm 0.01}{\sqrt{E}} \oplus 0.022 \pm 0.001
$$

For electromagnetic showers the Poisson width is

$$
\frac{\sigma(E)}{E}=\frac{0.499 \pm 0.008}{\sqrt{E}} \oplus 0.042 \pm 0.002
$$

In charged-current interactions the muon deposits energy in the shower region (about 1 MIP per counter on average), which must be subtracted from the pulseheight in $E_{c n t r}(i)$. The muon energy loss in MIP is subtracted by from the pulseheight using a parameterization of the restricted muon energy loss, $E_{\mu}^{\text {loss }}(i)$, (due to ionization) per counter in MIPs

$$
E_{\mu}^{\text {loss }}(i)=0.9315+0.02359 * \log \left(E_{\mu}^{e s t}\right)
$$

where $E_{\mu}^{e s t}$ is the estimated energy of the muon in GeV. The hadron energy is defined as the sum

$$
E_{H A D}=C_{\pi} \sum_{i=P L A C E}^{S H E N D-5}\left(E_{\text {cntr }}(i)-E_{\mu}^{\text {loss }}(i)\right),
$$

where PLACE is the most upstream counter of two consecutive counters with at least 4MIPs energy deposition. PLACE corresponds to the first counter downstream of the neutrino interaction vertex. SHEND is the counter downstream of PLACE, which is the first of three consecutive counters with less than 4MIPs energy deposition. This definition of the hadron energy differs from the definition used in reference [15] to calibrate and obtain resolution functions

$$
E_{H A D}=C_{\pi} \sum_{i=P L A C E}^{P L A C E-19} E_{c n t r}(i) .
$$

With $E_{H A D}$ definition 3.7 the calibration constant is the same, $C_{\pi}=0.212 \mathrm{GeV} / \mathrm{MIP}$. However, the mean of the ratio of test beam momentum to measured hadron energy for 75 $\mathrm{GeV}$ test beam hadrons is $0.997 \pm 0.001$, and the non-linearity factor is $\frac{e}{h}=1.099 \pm 0.011$. The width of the Poisson-like resolution function is now

$$
\frac{\sigma(E)}{E}=\frac{0.89 \pm 0.01}{\sqrt{E}} \oplus 0.021 \pm 0.001 .
$$




\subsection{MUON ANGLE RECONSTRUCTION}

The angle of the muon at the vertex is measured from the track by fitting the hits from the muon in the drift chambers of the target. Muons are highly penetrating particles. A muon traversed the whole target calorimeter loses roughly $14 \mathrm{GeV}$ of energy on average and its track will be almost a straight line in the target calorimeter. However, multiple Coulomb scattering affects the track reconstruction. MCS depends on both the length of the track and the muon momentum. The tracking algorithm fits for straight-line segments in the most downstream drift chambers of the target. The fitted line is extrapolated back to the vertex of the interaction (PLACE). The algorithm searches for hits which can be associated with the extrapolated line. The muon angle at the vertex is obtained by fitting the track hits close to the vertex. However, due to the presence of hadron shower, the hits in a number of chambers immediately after PLACE are not used in the track fit. The number of chambers omitted from the fit depends on the hadron energy. Showers with higher energy will traverse more chambers longitudinally. The uncertainty of the muon angle depends on the hadron energy and the uncertainty due to MCS. The dependence of MCS on momentum correlates the muon angle and momentum (discussed in next section) measurements. Therefore, the track fitting procedure is iterated. A GEANT based hit level Monte Carlo is used to parameterize the angular resolution function (see Chapter 4).

\subsection{MUON ENERGY RECONSTRUCTION AND CALIBRATION}

The event sample is divided into two categories depending on the length of the muon track:

- TOROID EVENTS are events for which the muon enters the toroid and produces hits in at least one of the gaps. This sample can be divided into "GAP 1" events, for which the muon either stops or leaves the toroid after prodicing hits in the first gap, or "TWO-GAPS" events, which produce hits in at least 2 gaps.

- TARGET EVENTS are events for which the muon does not enter the toroid. These are subdivided into "RANGEOUTS", which contain a muon stopping in the target and 
"EXITS", which contain a muon that exits before counter 1 without stopping.

This analysis uses only the 'TWO-GAPS' events sample.

\subsubsection{Muon Momentum in the Toroid}

Muon momentum in the toroid is measured from the muon track curvature in the magnetic field of the toroid. The bend angle, $\theta$, in a constant magnetic field in vacuum is

$$
\theta=\frac{0.3 B L}{P}
$$

where $P$ is the muon momentum, $L=R \theta$ is the length of the track in the magnetic field, and $R$ is the curvature of the track. In the toroid, the muon energy loss in steel and MCS must also be accounted for to measure the muon momentum. Muon momentum, as it enters

the toroid, $P_{\mu}^{F F}$, is obtained by the following algorithm: Link segments are found in all five sets of drift chambers in the toroid. Hits in the toroid chambers are fit to the projected track which is obtained by projecting the muon through the toroid using the muon angle measured in the target and a model of the magnetic field. Muon energy loss and MCS are also taken into account in the fit.

\subsubsection{Total Muon Energy}

Muons produced in neutrino interactions in the target lose part of their energy before entering the toroid where their momentum is analyzed. In the target, energy loss is calculated in two pieces: energy loss within the shower region and loss beyond the end of the shower. The shower region in an event is defined as the counters between PLACE and SHEND. The muon energy loss downstream of the shower region is calculated from the sum of the map corrected PH, (converted to $\mathrm{GeV}$ ), starting for counters six counters downstream of SHEND to the end of the target (counter number 1)

$$
E_{\mu}^{\text {target }}=\sum_{i=1}^{S H E N D-6} E_{c n t r}^{\mu}(i) .
$$

The muon energy deposited in counter $i, E_{c n t r}^{\mu}(i)$, is reconstructed from the counter PH using the following algorithm described below. "TCUT" is defined as the counter PH below which 
$C_{\mu}\left(E_{\mu}\right)$ (a "low energy" conversion factor) is used, and above which $C_{e}$ (a "high energy" conversion factor) is used to convert counter MIPs to GeV. A "low energy" deposition is attributed primarily to ionization loss. TCUT is set to $4.5 \mathrm{ADC}$ counts. For $E_{c n t r}(i)<$ TCUT the energy deposited in this counter by the muon in $\mathrm{GeV}$ is

$$
\begin{aligned}
E_{c n t r}^{\mu}(i) & =C_{\mu}\left(E_{\mu}\right) E_{c n t r}(i) \\
C_{\mu}\left(E_{\mu}\right) & =0.1419+0.004357 \log \left[\min \left(E_{\mu}^{e s t}, 60 .\right)\right]+ \\
& +0.00006822 \min \left(\left(E_{\mu}^{e s t}-60\right), 0\right) .
\end{aligned}
$$

A "high energy" deposition $(P H(i)>T C U T)$ is attributed to catastrophic energy loss which triggers an electromagnetic cascade over several counters. The conversion factor for pure electromagnetic cascade, $C_{e}$, is used in this case. The energy of the counter in $\mathrm{GeV}$ is given by

$$
E_{c n t r}^{\mu}(i)=C_{\mu}\left(E_{\mu}\right)+C_{e}\left(E_{c n t r}(i)-1 .\right)
$$

where $C_{e}=0.195 \mathrm{GeV} / \mathrm{MIP}$ and $C_{\mu}\left(E_{\mu}\right)$ is given in Equation 3.12. The first term in Equation 3.13 corresponds to the average muon ionization energy (1MIP) in GeV, therefore, 1 MIP is subtracted from the PH in the second term.

The energy deposited by the muon can not be separated from the shower energy in the shower region. A parameterization of the most probable value (MPV) of the muon energy loss is used to estimate the energy loss $E_{\mu}^{\text {shower }}$ in the shower region

$$
E_{\mu}^{\text {shower }}=\sum_{i=S H E N D-5}^{P L A C E}\left(0.1668+0.00037 E_{\mu}^{\text {est }}(i)\right) / \cos \left(\theta_{\mu}\right)
$$

where $\theta_{\mu}$ is the muon angle and $E_{\mu}^{e s t}(i)$ is the estimated muon energy.

The total muon energy is given by the sum of all three contributions

$$
E_{\mu}=E_{\mu}^{F F}+E_{\mu}^{\text {shower }}+E_{\mu}^{\text {target }}
$$




\subsubsection{Muon Energy Calibration}

Calibration of the muon energy was performed using $50 \mathrm{GeV}$ test beam muons to determine the overall normalization and the radial dependence of the magnetic field. The entire face of the toroid was mapped with $50 \mathrm{GeV}$ test beam muons. Event selection requirements were: hits in the drift chambers in all 3 gaps of the toroid, a well defined track in the target, and to spend the whole time in the steel (excluding events which spent any fraction of time in the crack or the central region of the toroid). In addition, all events were required to have a good track and reconstruction in the test beam spectrometer. A total of 45 test beam runs were used in the calibration analysis. Figure 3.4 shows the location of the beam at the front face of the toroid for the good test beam map runs.

For each run the fitted mean of the ratio $p^{T B} / p^{T O R}$ is computed, where $p^{T B}$ is the muon momentum measured by the test beam spectrometer and $p^{T O R}$ is muon momentum measured by the $\mathrm{NuTeV}$ detector. A correction to the magnetic field map is obtained as described below. For each of the 45 test beam runs we project two "average" tracks. Each "average" track is obtained by stepping the muon through the toroid. For each run the average front face position for a muon in the run and the average muon angle are computed and are used as the track initial position and angle. Average muon energy loss is taken into account and projected hits are simulated in all 3 gaps of the toroid.

The first "true" track for run $i$ projects a muon with energy $E_{\mu}^{F F}\left(=\left\langle E^{T B}\right\rangle_{i}-\left\langle E^{\text {loss }}\right\rangle\right)$ using the default magnetic field map from ANSYS, where $\left\langle E^{T B}\right\rangle_{i}$ is the truncated mean of the distribution $p^{T B}$ for run $i$ and $\left\langle E^{\text {loss }}\right\rangle$ is the average muon energy loss in the target. "True hits" $x_{i j}^{\text {true }}$ are produced for all gaps $j=1,3$. The second track for run $i$ projects a muon with energy $\left(\left\langle E^{T B}\right\rangle_{i} /\left(\left\langle p_{i}^{T B} / p_{i}^{T O R}\right\rangle-\left\langle E^{\text {loss }}\right\rangle\right)\right.$. For this track a position dependent multiplicative correction of the form $\operatorname{Cor}\left(R_{F F}\right)=a+b R_{F F},\left(R_{F F}=\sqrt{x_{F F}^{2}+y_{F F}^{2}}\right.$ is the radius at the front face of the toroid), is applied to the default magnetic field map. "Corrected hits" $x_{i j}^{\text {corr }}(a, b)$ are produced in each gap. The map correction parameters $a$ and $b$ are obtained by minimizing the difference between true and corrected hits in all gaps over all test beam runs

$$
\chi^{2}=\sum_{i=1}^{45} \sum_{j=1}^{3} \frac{\left(x_{i j}^{\text {true }}-x_{i j}^{\text {corr }}(a, b)\right)^{2}}{\sigma_{i}^{2}} .
$$




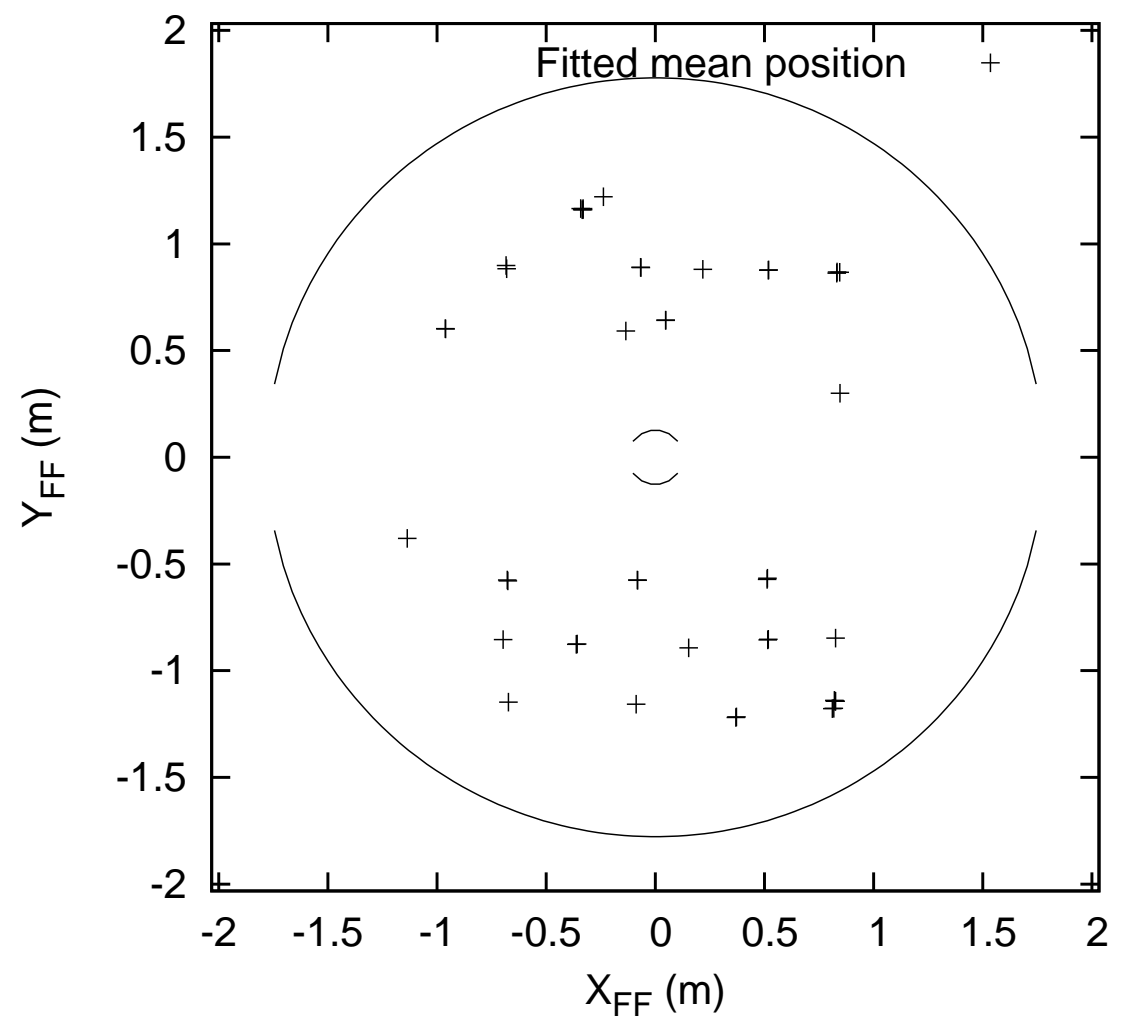

Figure 3.4: Fitted mean position at the front face of the toroid for all 45 muon test beam runs. 
The uncertainty of the absolute muon energy scale is taken to be the variance of the fitted means of $p_{T B} / p_{T O R}$ for all 45 map test beam runs after the calibration is applied to the magnetic field. The event-by-event uncertainty of the test beam spectrometer $(0.3 \%)$ is much smaller than the resolution of the toroid and is therefore neglected. The absolute muon energy scale uncertainty is found from this procedure to be $0.7 \%$.

\subsubsection{Muon Energy Resolution Function}

Muon momentum is measured from the track curvature in the toroid (Equation 3.10). The dominating uncertainty is due to MCS, which introduces uncertainty in the bend angle measurement $\sigma_{\theta}^{M C S} \propto 1 / p_{\mu}$. Therefore, the contribution to the resolution function from MCS given by $\sigma_{\theta}^{M C S} / \theta$ is a constant, because $\theta \propto 1 / p \mu$. In addition, the uncertainty due to chamber resolution $\sigma_{\theta}^{C R}$ is a constant which becomes important at relatively high energy. The combined resolution function will have the form

$$
\frac{\sigma_{\theta}}{\theta}=\frac{\sigma_{\theta}^{M C S}}{\theta} \oplus \frac{\sigma_{\theta}^{C R}}{\theta}=A \oplus B \cdot p_{\mu}
$$

where $A$ is determined by MCS and $B$ by chamber resolution. Therefore the momentum res-

olutions function will have the simple form $\frac{\Delta(1 / P)}{(1 / P)}$. Catastrophic energy loss and hard single scattering processes in the toroid are responsible for the asymmetric tails in the resolution function distributions. Test beam muons with energies ranging from 30 to $170 \mathrm{GeV}$ are used to parameterize the resolution function of the muon spectrometer, using a fit of the form:

$$
\frac{\Delta\left(1 / P_{\mu}\right)}{\left(1 / P_{\mu}\right)}=\exp \left[-\frac{1}{2} \frac{x^{2}}{\sigma_{\text {lead }}^{2}\left(P_{\mu}\right)}\right]+R_{\text {tail }}\left(P_{\mu}\right) \exp \left[-\frac{1}{2} \frac{\left(x-x_{\text {tail }}\left(P_{\mu}\right)\right)^{2}}{\sigma_{\text {tail }}^{2}\left(P_{\mu}\right)}\right]
$$

where $\sigma_{\text {lead }}\left(P_{\mu}\right)$ is the width of the leading Gaussian contribution due to MCS, and $R_{\text {tail }}\left(P_{\mu}\right)$, $\sigma_{\text {tail }}\left(P_{\mu}\right)$ and $x_{\text {tail }}\left(P_{\mu}\right)$ are the normalization coefficient, the width and the offset of the asymmetric tail of the resolution function. The width of the leading Gaussian is parameterized with the following function according to Equation 3.17:

$$
\sigma_{\text {lead }}\left(P_{\mu}\right)=\sqrt{A^{2}+\left(B P_{\mu}\right)^{2}}
$$


where $A$ and $B$ are constants, determined from a fit. $R_{\text {tail }}\left(P_{\mu}\right), \sigma_{\text {tail }}\left(P_{\mu}\right)$, and $x_{\text {tail }}\left(P_{\mu}\right)$ are parameterized with linear functions

$$
\begin{aligned}
& R_{\text {tail }}\left(P_{\mu}\right)=R_{\text {tail }}^{0}+R_{\text {tail }}^{1} P_{\mu}, \\
& \sigma_{\text {tail }}\left(P_{\mu}\right)=\sigma_{\text {tail }}^{0}+\sigma_{\text {tail }}^{1} P_{\mu}, \\
& x_{\text {tail }}\left(P_{\mu}\right)=x_{\text {tail }}^{0}+x_{\text {tail }}^{1} P_{\mu} .
\end{aligned}
$$

The resolution function given by Equation 3.18 is fitted to the distribution

$$
\frac{\Delta\left(1 / P_{\mu}\right)}{\left(1 / P_{\mu}\right)}=\frac{1 / P_{\mu}^{D E T}-1 / P_{\mu}^{T B}}{1 / P_{\mu}^{T B}}=\frac{P_{\mu}^{T B}-P_{\mu}^{D E T}}{P_{\mu}^{D E T}}
$$

where $P_{\mu}^{D E T}$ and $P_{\mu}^{T B}$ are the muon momentum measured by $\mathrm{NuTeV}$ detector and the test beam respectively. The parameters determined from the fit are $A=0.102, B=$ $0.42 \times 10^{-3} \mathrm{GeV}^{-1}, R_{\text {tail }}^{0}=0.79 \times 10^{-2}, \sigma_{\text {tail }}^{0}=0.45, \sigma_{\text {tail }}^{1}=1.3 \times 10^{-4} \mathrm{GeV}^{-1}$, and $x_{\text {tail }}^{0}=0.19$. $R_{\text {tail }}^{1}$ and $x_{\text {tail }}^{1}$ are consistent with 0 . For a muon entering the toroid with momentum $P_{\mu}=100 \mathrm{GeV}$ the resolution function parameters have the following values $\sigma_{\text {lead }}(100)=0.11$, $\sigma_{\text {tail }}(100)=0.46, x_{\text {tail }}(100)=0.19$, and $R_{\text {tail }}(100)=0.079$. Figure 3.5 shows the response of the detector to test beam muons with incident energy of $100 \mathrm{GeV}$ (points) and the fit using the parameterization from Equation 3.20 (curve).

An alternative (linear) parameterization was used for the width of the leading Gaussian

$$
\sigma_{\text {lead }}\left(P_{\mu}\right)=C+D P_{\mu}
$$

to examine the difference in extrapolation of the function to high energy beyond the test beam data. The fit quality was equally good. The parameters obtained were $C=0.097$ and $D=1.2 \times 10-4 \mathrm{GeV}^{-1}$. Figure 3.6 shows both parameterizations of $\sigma_{\text {lead }}\left(P_{\mu}\right)$ as function of muon momentum. Both parameterizations perform equally well in the region of test beam data, but the high energy extrapolation is different. We use this difference as a systematic uncertainty in our analysis. 


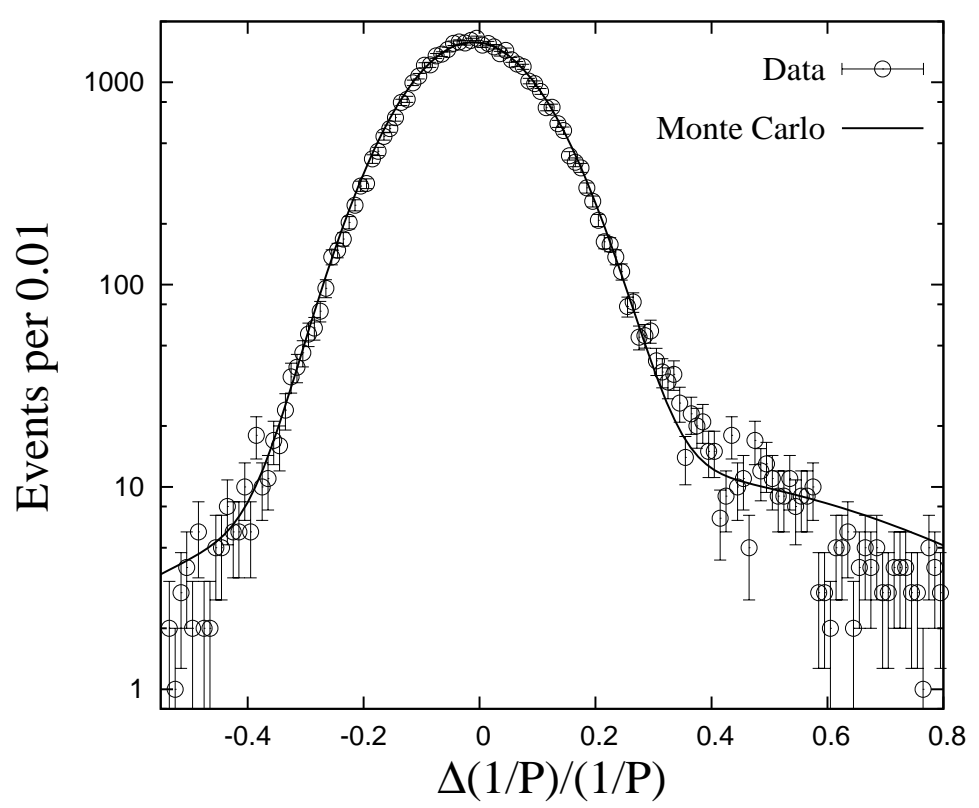

Figure 3.5: Detector response to test beam muons with incident energy of $100 \mathrm{GeV}$ (points) and the fit (curve).

\subsection{EVENT SELECTION AND DATA SAMPLES}

The are several types of selection criteria used in defining the data samples. The remainder of this chapter provides a description of the selection criteria and defines each data sample used in this analysis.

\subsubsection{Quality and Fiducial Volume Cuts}

This set of cuts selects events whose vertex was contained well inside the volume of the detector and which were obtained during periods when the detector and the beamline were fully operational.

- BAD RUNS - Removes runs for which detector hardware was either non-operational or poorly understood.

- IGATE - Selects the neutrino gate. 


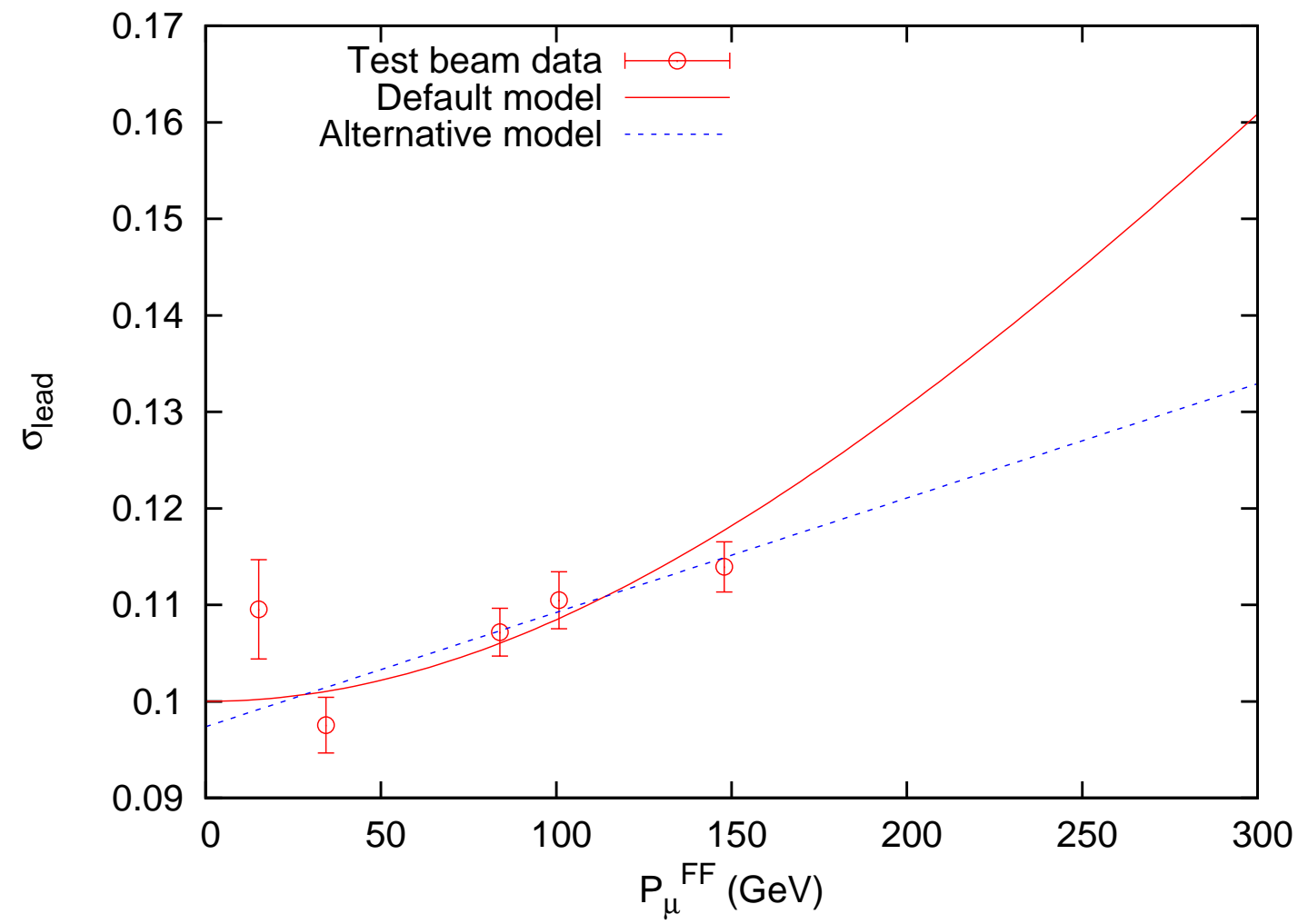

Figure 3.6: Energy dependence of the width of the leading Gaussian. The $\mathrm{x}$ axis is the mean muon energy $\left[p_{\mu}^{F F}\right]$ at the front face of the toroid. 
- NSTIME - Selects single interaction events by requiring the muon track time to be within $36 \mathrm{~ns}$ of the hadron shower time.

- PLACE - Requires $20<P L A C E<80$ to ensure longitudinal containment of the shower within the calorimeter.

- VERTEX BOX - Requires $V_{x}<1.27 m$ and $V_{y}<1.27 m$ to ensure transverse containment of the hadron shower.

\subsubsection{Tracking and Geometric Cuts}

These cuts ensure good acceptance of the detector and quality of the muon track.

- TRIGGER 1 - Selects events with muon track which starts in the calorimeter and has produced hits in the toroid drift chambers. These events are charged-current candidates.

- TARGET TRACK - Requires a good muon track in the target, which is important for an accurate muon angle measurement.

- TOROID CONTAINMENT - Requires the radius at the front face (FF) of the toroid to be $R_{F F}=\sqrt{X_{F F}^{2}+Y_{F F}^{2}}<1.62 m$, where $X_{F F}, Y_{F F}$ are the coordinates of the track at the FF. Also requires the radius of the fitted track in the first gap (T2) of the toroid to be $R_{T 2}=\sqrt{X_{T 2}^{2}+Y_{T 2}^{2}}<1.35 m$. This ensures good acceptance of the muon track in the toroid and constrains the trajectory of the muon to well understood regions.

- TOROID TRACK - Requires a minimum number of hits in the toroid drift chambers to ensure good reconstruction of the muon track in the toroid.

- HOLE CUT - Requires the radius of the projected track from the vertex to be more than $15.2 \mathrm{~cm}$ at the FF of the toroid and the muon angle at the vertex $\theta_{\mu}>0.007 \mathrm{rad}$. This ensures the muon will not travel in the toroid hole where the magnetic field (in air) is poorly modeled.

- $\mathbf{8 0 \%}$ STEEL - Requires that the muon track spend more than $80 \%$ of its toroid trajectory in steel where the magnetic field is well known.

- GOOD FIT - Requires a fitted muon track in the toroid. The number of degrees of freedom are the number of chambers which have hits. 
- TWO GAPS - Requires the muon track to have hits in both the first and second gap drift chambers. This ensures a track of adequate length to obtain a well measured momentum.

\subsubsection{Total Cross Sample}

The total cross section sample is used to normalize the neutrino flux and determine the anti-neutrino to neutrino cross section ratio, defined later. This data sample requires all the above selection criteria with two additional cuts:

- MUON ANGLE - Requires $\theta_{\mu}<0.15 \mathrm{rad}$ to ensure good acceptance in the toroid.

- MUON ENERGY - Requires $15<E_{\mu}<600 \mathrm{GeV}$ and $E_{\mu F F}>3 \mathrm{GeV}$. If the energy of the muon is very high, the determination of the track curvature is limited by drift chamber resolution. In addition, the number of hits from catastrophic energy loss, which rises linearly with energy, will affect the tracking algorithm. Events with $E_{\mu}<15 \mathrm{GeV}$ are removed, because at very low energy $(<15 \mathrm{GeV})$ MCS reduces the reconstruction efficiency.

\subsubsection{Flux Sample}

The flux sample is used to determine the relative neutrino flux (shape with $E_{\nu}$ ) from a subsample of the total cross section sample with the additional requirement of a hadronic energy cut:

- HADRON ENERGY - Requires $E_{H A D}<20 \mathrm{GeV}$.

\subsubsection{Structure Function Sample}

The structure function sample is used to extract the DIS differential cross section. It is a subsample of the total cross section sample with additional kinematic requirements:

- HADRON ENERGY - Requires $10<E_{H A D}<600 \mathrm{GeV}$. Events with very low hadron energy are removed to minimize the correlations with the flux sample and to remove "quasi-elastic" and resonances events. 
- $Q^{2}$ CUT - Requires $Q^{2}>0.5 \mathrm{GeV}^{2} / c^{2}$. Reduces the contribution from "quasi-elastic" events.

- NEUTRINO ENERGY - Requires $30<E_{H A D}<360 \mathrm{GeV}$.

- DIMUON CUT - Removes events with more than one muon track. 


\subsection{MONTE CARLO EVENT SIMULATOR}

An ideal detector would give the true value (TRUTH) for each measurable physics quantity. In real detectors the values of the physics quantities are smeared due to resolution effects; therefore we measure "SMEARED" kinematic quantities. The physical size of the detector also limits the "measurable events" to those contained in the defined geometry of the detector. As a consequence real detectors give the SMEARED value of the physics quantities in a kinematic region which is limited by the acceptance of the detector. The Monte Carlo simulation is used to determine a correction which can convert a SMEARED kinematic distribution measured in the detector to a TRUTH distribution. This chapter discusses the $\mathrm{NuTeV}$ Monte Carlo event simulation.

NUMONTE is the Monte Carlo event simulator used by $\mathrm{NuTeV}$ to correct data. It consists of: 1) an event generator which generates neutrino interaction events according to an incident neutrino flux and cross section model, and 2) a detector simulator which models the response of the detector components for each generated event. The event generator calculates the TRUTH kinematic variables for an inclusive neutrino interaction, and then processes them through a detector model. NUMONTE doesn't perform a detailed simulation of all final state particles interacting in the detector. Instead, it uses a parameterization of the detector response obtained from test beam data to smear the TRUTH kinematic variables. Cosmic rays and beam muons are not modeled because they are removed from data. 


\subsection{CROSS SECTION MODEL}

The charged-current DIS cross section model implements the differential cross section given by Equation 1.18, which follows the V-A structure of the weak interaction

$$
\frac{d^{2} \sigma^{\nu(\bar{\nu})}}{d x d y}=\frac{G_{F}^{2} M E_{\nu}}{\pi\left(1+\frac{Q^{2}}{M_{W}^{2}}\right)^{2}}\left[\frac{y^{2}}{2} 2 x F_{1}\left(x, Q^{2}\right)+(1-y) F_{2}\left(x, Q^{2}\right) \pm\left(y-\frac{y^{2}}{2}\right) x F_{3}\left(x, Q^{2}\right)\right] .
$$

In leading order QCD the structure functions are expressed in terms of the quark densities by

$$
\begin{aligned}
2 x F_{1}\left(x, Q^{2}\right) & =\left[x u_{v}\left(x, Q^{2}\right)+x d_{v}\left(x, Q^{2}\right)\right. \\
& \left.+2 x u_{s}\left(x, Q^{2}\right)+2 x d_{s}\left(x, Q^{2}\right)+2 x s_{s}\left(x, Q^{2}\right)\right] \\
F_{2}\left(x, Q^{2}\right) & =2 x F_{1}\left(x, Q^{2}\right) \times \frac{1+R\left(x, Q^{2}\right)}{1+4 M^{2} x^{2} / Q^{2}}, \\
x F_{3}\left(x, Q^{2}\right) & =x u_{v}\left(x, Q^{2}\right)+x d_{v}\left(x, Q^{2}\right) .
\end{aligned}
$$

The foundation of the cross section model are the parton distribution functions. Here, $u_{v}, d_{v}, u_{s}, d_{s}$ and $s_{s}$ are the PDFs for an iron target. There are two types of PDF parameterizations that can be used

1. Global fit - a parameterization which is available from a global fit to world data. These are available from theoretical collaborations which perform QCD-based global fits. These PDFs are determined for the proton and neutron. For us to use such a parameterization for our neutrino iron PDFs we must assume a nuclear correction. This presents a problem because nuclear corrections have not been measured in neutrino scattering. In addition, a particular global fit parameterization may not describe $\mathrm{NuTeV}$ data. This affects the acceptance correction used to extract the flux and the differential cross section.

2. NuTeV Data fit - a parameterization which is a fit to our data. This approach uses an iterative procedure to obtain a model which describes $\mathrm{NuTeV}$ data. By fitting our data directly nuclear effects are automatically accounted for in the PDFs, and the result, by construct, models our data well.

This analysis uses the second approach, which gives more flexibility and involves fewer assumptions. 


\subsubsection{PDF Parameterization}

The cross section model is based on a leading order parton distribution parameterization from Buras and Gaemers (BG) [18]. Quark distributions are parameterized as functions of $x$ at fixed $Q_{0}^{2}=12.6 \mathrm{GeV}^{2} / c^{2}$ (NuTeV's average $\left.Q^{2}\right)$. The quark distributions are then evolved to any $Q^{2}$. The original BG model uses the following parameterization:

- Valence - Valence distributions are of the form $x^{E_{1}}(1-x)^{E_{2}}$. They are constrained so that the valence quarks are 0 at both $x=0$ and $x=1$. The d-quark function falls faster than the u-quark function in the limit $x \rightarrow 1$. Hence the softer d-valence is parameterized as $d_{v}=u_{v}(1-x)$.

- Light quark sea - Sea distributions are parameterized using the form $(1-x)^{E S}$. This gives a rising function as $x \rightarrow 0$ and at high $\mathrm{x}$ the sea terms are suppressed. The model assumes the symmetry $\bar{u}=\bar{d}$. This assumption is modified to agree with existing data which finds an asymmetry (see below).

- Heavy quark sea - The model assumes strange sea symmetry, $s=\bar{s}$. The parameterization allows the strange sea to have a different shape than light quark sea by including a factor $(1-x)^{\alpha}$. The charm sea is neglected.

- Gluon - Since the model is LO, the gluon distribution is not directly parameterized. The gluon distribution appears through its moments as missing quarks by satisfying the sum rules (discussed later).

An enhanced BG model is used for greater flexibility, by adding terms. The parton distributions are parameterized as follows:

$$
\begin{aligned}
x u_{v}(x) & =u_{t o t} \times\left[x^{E_{10}}(1-x)^{E_{20}}+A V_{2} x^{E_{30}}(1-x)^{E_{40}}\right], \\
x d_{v}(x) & =d_{t o t} \times x u_{v}(x) \times(1-x), \\
x \bar{u}(x) & =x \bar{d}(x)=\frac{1}{2(\kappa+2)} x S(x) \frac{1}{2(\kappa+2)}\left(A S(1-x)^{E S}+A S_{20}(1-x)^{E S_{20}}\right), \\
x s(x) & =\bar{x} s(x)=\frac{\kappa}{2(\kappa+2)} x S S(x) \frac{\kappa}{2(\kappa+2)} \frac{A S}{1+E S}(E S+\alpha+2)(1-x)^{(E S+\alpha)} .
\end{aligned}
$$

The following symmetries are assumed to reduce the number of free parameters: 


\section{- Isospin symmetry}

$$
\begin{array}{ll}
d(x) \equiv d^{p}(x)=u^{n}(x) & , \bar{d}(x) \equiv \bar{d}^{p}(x)=\bar{u}^{n}(x), \\
u(x) \equiv u^{p}(x)=d^{n}(x) & , \bar{u}(x) \equiv \bar{u}^{p}(x)=\bar{d}^{n}(x), \\
s(x) \equiv s^{p}(x)=s^{n}(x) & , \quad \bar{s}(x) \equiv \bar{s}^{p}(x)=\bar{s}^{n}(x), \\
c(x) \equiv c^{p}(x)=c^{n}(x) & , \quad \bar{c}(x) \equiv \bar{c}^{p}(x)=\bar{c}^{n}(x) .
\end{array}
$$

\section{- Sea symmetries}

$$
\begin{aligned}
\bar{u} & =\bar{d}, \\
s & =\bar{s}, \\
c & =\bar{c} .
\end{aligned}
$$

4.1.1.1 Valence Evolution Valence distributions at any $Q^{2}$ are given by

$$
\begin{aligned}
& x u_{v}\left(x, Q^{2}\right)=u_{t o t}\left(Q^{2}\right) \times\left[x^{E_{1}}(1-x)^{E_{2}}+A V_{2} x^{E_{3}}(1-x)^{E_{4}}\right] \\
& x d_{v}\left(x, Q^{2}\right)=d_{t o t}\left(Q^{2}\right) \times x u_{v}\left(x, Q^{2}\right) \times(1-x) .
\end{aligned}
$$

The exponents $\left(E_{1}, E_{2}, E_{3}, E_{4}\right)$ vary with $Q^{2}$ according to the original form proposed by Buras and Gaemers

$$
\begin{aligned}
& E_{1}=E_{10}+E_{11} \cdot s, \\
& E_{2}=E_{20}+E_{21} \cdot s, \\
& E_{3}=E_{30}+E_{11} \cdot s, \\
& E_{4}=E_{40}+E_{21} \cdot s,
\end{aligned}
$$

where

$$
s=\log \left[\frac{\log \left(\frac{Q^{2}}{A_{0}^{2}}\right)}{\log \left(\frac{Q_{0}^{2}}{A_{0}^{2}}\right)}\right] .
$$


The form of $s$ is chosen, so that $s=0$ at $Q_{0}^{2}=12.6 \mathrm{GeV}^{2} / \mathrm{c}^{2}$. Valence normalizations are obtained from the GLS sum rule which gives the total number of valence quarks

$$
\begin{aligned}
\int_{0}^{1} x F_{3}\left(x, Q^{2}\right) \frac{d x}{x} & =\int_{0}^{1}\left(x u_{v}\left(x, Q^{2}\right)+x d_{v}\left(x, Q^{2}\right)\right) \frac{d x}{x} \\
& =3\left(1-\frac{\alpha_{s}\left(Q^{2}\right)}{\pi}\right)=3\left[1-\frac{A_{1}}{\log \left(Q^{2} / A_{0}^{2}\right)}-\frac{A_{2}}{\left[\log \left(Q^{2} / A_{0}^{2}\right)\right]^{2}}\right]
\end{aligned}
$$

This normalization varies according to the QCD parameter, $A_{0}$, which parameterizes the effect of $\Lambda_{Q C D}$ and represents the strength of the scaling violations. The relative normalization between $u_{v}$ and $d_{v}$ is constrained

$$
\int_{0}^{1} u_{v}\left(x, Q^{2}\right) d x=2 \int_{0}^{1} d_{v}\left(x, Q^{2}\right) d x=2\left[1-\frac{A_{1}}{\log \left(Q^{2} / A_{0}^{2}\right)}-\frac{A_{2}}{\left[\log \left(Q^{2} / A_{0}^{2}\right)\right]^{2}}\right]
$$

Hence, the normalization $u_{t o t}\left(Q^{2}\right), d_{t o t}\left(Q^{2}\right)$ are

$$
\begin{aligned}
u_{t o t}\left(Q^{2}\right)= & 2 \frac{\left[1-\frac{A_{1}}{\log \left(Q^{2} / A_{0}^{2}\right)}-\frac{A_{2}}{\left[\log \left(Q^{2} / A_{0}^{2}\right)\right]^{2}}\right]}{\beta\left(E_{1}, E_{2}+1\right)+A V_{2} \beta\left(E_{3}, E_{4}+1\right)} \\
d_{t o t}\left(Q^{2}\right)= & \frac{\left[1-\frac{A_{1}}{\log \left(Q^{2} / A_{0}^{2}\right)}-\frac{A_{2}}{\left[\log \left(Q^{2} / A_{0}^{2}\right)\right]^{2}}\right]}{\beta\left(E_{1}, E_{2}+2\right)+A V_{2} \beta\left(E_{3}, E_{4}+2\right)} .
\end{aligned}
$$

where $\beta(m, n)=\frac{\Gamma(m) \Gamma(n)}{\Gamma(m+n)}$ is Euler's $\beta$ function. In addition a charge constraint is required

$$
\begin{aligned}
\frac{2}{3} \int_{0}^{1} u_{v}\left(x, Q^{2}\right) d x & -\frac{1}{3} \int_{0}^{1} d_{v}\left(x, Q^{2}\right) d x \\
& =\left[1-\frac{A_{1}}{\log \left(Q^{2} / A_{0}^{2}\right)}-\frac{A_{2}}{\left[\log \left(Q^{2} / A_{0}^{2}\right)\right]^{2}}\right] .
\end{aligned}
$$

Here $A_{0}, A_{1}, A_{2}, A V_{2}, E_{10}, E_{20}, E_{30}, E_{40}, E_{11}, E_{12}$ are parameters varied in cross section model fit. 
4.1.1.2 Light Sea Evolution The light sea distribution at any $Q^{2}$ is

$$
\begin{aligned}
x \bar{u}\left(x, Q^{2}\right) & =x \bar{d}\left(x, Q^{2}\right)=\frac{1}{2(\kappa+2)} x S\left(x, Q^{2}\right) \\
& =\frac{1}{2(\kappa+2)}\left(A S(1-x)^{E S}+A S_{2}(1-x)^{E S_{2}}\right) .
\end{aligned}
$$

The $Q^{2}$ evolution of the light quark sea is controlled by the second and the third moments because they decrease rapidly with $x$. The ' $n-t h$ ' moment of the light sea distribution in $x$ is defined as

$$
S Q_{n}=\int_{0}^{1} x^{n-1} S(x) d x .
$$

A QCD-like calculation constrains the second and the third moment, but they are allowed to vary. $S Q_{2}$ and $S Q_{3}$ are calculated for 4-flavor $\mathrm{SU}(3)$ gauge theory

$$
\begin{aligned}
& S Q_{2}=\int_{0}^{1} x S d x=\frac{3}{4} D_{22}+\frac{1}{4} D_{12} \\
& S Q_{3}=\int_{0}^{1} x^{2} S d x=\frac{3}{4} D_{23}+\frac{1}{4} D_{13} .
\end{aligned}
$$

The values for $D_{i j}$ are completely specified by LO QCD and are given by

$$
\begin{aligned}
D_{12} & =S_{2} e^{-0.427 \cdot s}, \\
D_{13} & =S_{3} e^{-0.667 \cdot s}, \\
D_{22} & =\left[(1-0.429)\left(S_{2}+V_{82}\right)-0.429 \cdot G_{2}\right] e^{-0.747 \cdot s} \\
& +\left[0.429\left(S_{2}+V_{82}\right)+0.429 \cdot G_{2}\right]-V_{82} e^{-0.427 \cdot s}, \\
D_{23} & =\left[(1-0.925)\left(S_{3}+V_{83}\right)-0.288 \cdot G_{3}\right] e^{-1.386 \cdot s} \\
& +\left[0.925\left(S_{3}+V_{83}\right)+0.288 \cdot G_{3}\right] e^{-0.609 \cdot s}-V_{83} e^{-0.667 \cdot s} .
\end{aligned}
$$

where the numerical values are taken from [18], and $G_{2}, G_{3}$ are the second and the third moment of the gluon distribution. The second moment of the gluon distribution $G_{2}=$ $\int_{0}^{1} x g\left(x, Q^{2}\right) d x$ is obtained from the momentum sum rule as missing momentum evaluated at $Q^{2}=Q_{0}^{2}$.

$$
G_{2}+\int_{0}^{1} \frac{1+R\left(x, Q_{0}^{2}\right)}{1+4 M^{2} x^{2} / Q_{0}^{2}}\left[x u_{v}\left(x, Q_{0}^{2}\right)+x d_{v}\left(x, Q_{0}^{2}\right)+x S\left(x, Q_{0}^{2}\right)\right] d x=1
$$


The sea normalizations, $V_{82}$ and $V_{83}$, are calculated from the moments of the valence distributions at $Q_{0}^{2}$

$$
\begin{aligned}
V_{82} & =u_{t o t}\left[\beta\left(E_{1}+1, E_{2}+1\right)+A V_{2} \beta\left(E_{3}+1, E_{4}+1\right)\right] \\
& +v_{t o t}\left[\beta\left(E_{1}+1, E_{2}+2\right)+A V_{2} \beta\left(E_{3}+1, E_{4}+2\right)\right], \\
V_{83} & =u_{t o t}\left[\beta\left(E_{1}+2, E_{2}+1\right)+A V_{2} \beta\left(E_{3}+2, E_{4}+1\right)\right] \\
& +v_{t o t}\left[\beta\left(E_{1}+2, E_{2}+2\right)+A V_{2} \beta\left(E_{3}+2, E_{4}+2\right)\right] .
\end{aligned}
$$

$A S_{2}$ and $E S_{2}$ are additional terms added to the original parameterization which are evolved with $Q^{2}$ in a semi-arbitrary way

$$
\begin{aligned}
& A S_{2}=A S_{20}+A S_{21} \log \left(Q^{2}\right), \\
& E S_{2}=E S_{20}+E S_{21} \log \left(Q^{2}\right) .
\end{aligned}
$$

$A S$ and $E S$ are constrained so that the second and the third moments match $S Q_{2}$ and $S Q_{3}$

$$
\begin{aligned}
E S & =\frac{S_{2}-A S_{2} /\left(E S_{2}+1\right)}{S_{3}-A S 2 /\left(\left(E S_{2}+1\right)\left(E S_{2}+2\right)\right)}-2, \\
A S & =(E S+1) \frac{S_{2}-A S_{2}}{E S_{2}+1} .
\end{aligned}
$$

Because both terms involving $A S, E S, A S_{2}$ and $E S_{2}$ are required to match the second and third moments, this calculation will deviate from 'QCD-like' starting at the fourth moment in $x$. Sea parameters included in the fit are $S_{2}, S_{3}, G_{3}, A S_{20}, A S_{21}, E S_{20}, E S_{21}$. 
4.1.1.3 Strange Sea Neutrino scattering dimuon data provides a direct measurement of the strange sea. The underlying leading order process is inclusive neutrino CC scattering with two oppositely charged muons in the final state given by

$$
\nu_{\mu}\left(\bar{\nu}_{\mu}\right)+\left(\begin{array}{l}
d(\bar{d}) \\
s(\bar{s})
\end{array}\right) \rightarrow \mu^{-(+)}+c(\bar{c})+X,
$$

where the charm quark decays semi-leptonically to an oppositely charged muon. Scattering off a $d$-quark is Cabibbo suppressed, because the CKM matrix element $\left|V_{c d}\right|^{2}=0.05$ is small [19]. As a result the scattering from $s$-quarks is a large contribution to the dimuon event sample.

The strange sea distribution was extracted in a leading-order dimuon analysis from CCFR dimuon data [20]. It was found that strange sea has different shape in $x$ than the non-strange sea. The following parameterizations were used for the non-strange sea,

$$
x S\left(x, Q^{2}\right)=A S(1-x)^{E S},
$$

and the strange sea,

$$
x S S\left(x, Q^{2}\right)=\frac{A S}{E S+1}(\beta+1)(1-x)^{E S+\alpha}=x S\left(x, Q^{2}\right) \frac{\beta+1}{E S+1}(1-x)^{\alpha},
$$

where $\beta=E S+\alpha$ describes the shape of strange sea. The strange sea is then normalized using

$$
\int_{0}^{1} x S\left(x, Q^{2}\right) d x=\int_{0}^{1} x S S\left(x, Q^{2}\right) d x
$$

which gives the relative normalization

$$
\kappa=\frac{2 S}{\bar{U}+\bar{D}}
$$

where $S=\int_{0}^{1} x s(x) d x, \bar{U}=\int_{0}^{1} x \bar{u}(x) d x, \bar{D}=\int_{0}^{1} x \bar{d}(x) d x$. As a result, the strange sea distribution obtains the form

$$
\begin{aligned}
x s(x) & =x \bar{s}(x)=\frac{\kappa}{2(\kappa+2)} x S S(x)=\frac{\kappa}{2(\kappa+2)} \frac{A S}{E S+1}(E S+\alpha+1)(1-x)^{E S+\alpha} \\
& =\frac{\kappa}{2(\kappa+2)} \frac{x \bar{u}(x)+x \bar{d}(x)}{2}(1-x)^{\alpha},
\end{aligned}
$$

The values of $\kappa$ and $\alpha$ are determined by a fit to the CCFR dimuon data and are given in Table 4.1. 
4.1.1.4 Modifications to the PDF parameterization Additional corrections are applied to the PDF parameterization to account for physics not present in our LO model. Where possible, existing data are used to constrain the model in kinematic regions where $\mathrm{NuTeV}$ has limited or no data.

- $d_{v} / u_{v}$ correction - Our PDF parameterization assumes $u(x)=d(x)$. An asymmetry is observed by the precise NMC $F_{2}^{d} / F_{2}^{p}$ data [21]. Neutrino scattering off an isocalar target is not sensitive to the asymmetry (actually $\mathrm{NuTeV}$ iron target has an $5.67 \%$ excess of neutrons). This asymmetry becomes important at high $\mathrm{x}$ where the $u_{v}$ and $d_{v}$ are quite different. A correction to the valence distributions obtained from NMC data is used to account for this effect. The ratio $F_{2}^{d} / F_{2}^{p}$ is calculated using the CCFR BG model [2] and corrected for nuclear effects. Comparison with $\mathrm{NMC} F_{2}^{d} / F_{2}^{p}$ data gives a correction which is parameterized as a polynomial

$$
\delta(d / u)=0.12079-1.3303 x+4.9829 x^{2}-8.4465 x^{3}+5.7324 x^{4} .
$$

The new ratio of the modified valence is

$$
\left(d_{v}^{\prime} / u_{v}^{\prime}\right)=\left(d_{v} / u_{v}\right)+\delta(d / u)
$$

where $u_{v}^{\prime}$ and $d_{v}^{\prime}$ are constrained by the measured $x F_{3}$ to satisfy $u_{v}^{\prime}+d_{v}^{\prime}=u_{v}+d_{v}$. Therefore the modified valence distributions obtain the form

$$
\begin{aligned}
u_{v}^{\prime} & =\frac{u_{v}}{1+\delta(d / u) \cdot u_{v} /\left(u_{v}+d_{v}\right)} \\
d_{v}^{\prime} & =\frac{d_{v}+u_{v} \cdot \delta(d / u)}{1+\delta(d / u) \cdot u_{v} /\left(u_{v}+d_{v}\right)}
\end{aligned}
$$

- $\bar{d} / \bar{u}$ correction - A large flavor asymmetry in the proton sea was observed by Drell-Yan data from E866 [22]. The light quark sea distributions are corrected with the factor

$$
f(\bar{d} / \bar{u})=\frac{1}{\max \left(1-x\left(2.7-0.14 \ln \left(Q^{2}\right)-1.9 x\right), 0.1\right)}
$$


which is obtained from a fit to E866 Drell-Yan data. The modified light sea distributions $\overline{u^{\prime}}, \overline{d^{\prime}}$ are constrained by $\overline{u^{\prime}}+\overline{d^{\prime}}=\bar{u}+\bar{d}$ and have the form:

$$
\begin{aligned}
\overline{u^{\prime}} & =\bar{u}\left(\frac{\bar{u}+\bar{d}}{\bar{u}+\bar{d} \cdot f(\bar{d} / \bar{u})}\right), \\
\overline{d^{\prime}} & =\bar{d}\left(\frac{\bar{u}+\bar{d}}{\bar{u}+\bar{d} \cdot f(\bar{d} / \bar{u})}\right) \cdot f(\bar{d} / \bar{u}) .
\end{aligned}
$$

- Low $Q^{2}$ extrapolation - BG PDFs are not well behaved in the region below $Q^{2}=$ $1 \mathrm{GeV}^{2} / \mathrm{c}^{2}$. Therefore an external PDF set is used to control the shape at low $Q^{2}$. The $Q^{2}$ shape of GRV94LO PDFs [23] is used to extrapolate the BG model down to $Q^{2}=$ $0.23 \mathrm{GeV}^{2} / \mathrm{c}^{2}$. GRV94LO is normalized to have the same value as BG model at $Q^{2}=$ $1.35 \mathrm{GeV}^{2} / \mathrm{c}^{2}$

- Higher Twist correction - Our PDF parameterization is constrained by a fit to the $\mathrm{NuTeV}$ differential cross section data. Through this fit some of the nonperturbative effects, present in data are absorbed by the parameterization. However, at high $x$ and low $Q^{2}$, there is limited $\mathrm{NuTeV}$ data to constrain the Higher Twist contribution (see Section 1.4.4.1). An empirical model is used to describe the $Q^{2}$ dependence in the high $x$ low $Q^{2}$ kinematic region [24]. This model rescales Bjorken $x$

$$
x \rightarrow \xi=x \frac{Q^{2}+B_{H T}}{Q^{2}+A_{H T} \cdot x} .
$$

Charge lepton measurements of $F_{2}^{d}$ and $F_{2}^{p}$ from SLAC [25], BCDMS [26] and NMC [27] above $x=0.4$ are used to constrain the higher twist parameters $A_{H T}$ and $B_{H T}$. This is achieved by including the charge lepton data in the PDF fit after applying correction for nuclear effects and 5/18th rule. This will be discussed in more detail in Section 4.1.5. 


\subsubsection{Longitudinal Structure Function}

A parameterization of $R_{L}$ is used to account for NLO processes at low $x . R_{L}$ is determined from an empirical fit to the combined SLAC proton and deutron scattering data [28].

$$
\begin{aligned}
R_{L}\left(x, Q^{2}\right) & =\frac{0.0635}{\ln \left(Q^{2} / 0.04\right)} \Theta\left(x, Q^{2}\right)+\frac{0.5747}{Q^{2}}-\frac{0.3534}{Q^{4}+0.09} \\
\Theta\left(x, Q^{2}\right) & =1+12\left(\frac{Q^{2}}{1+Q^{2}}\right)\left(\frac{0.125^{2}}{x^{2}+0.125^{2}}\right)
\end{aligned}
$$

This parameterization describes the data well for $0.07<x<0.7$ and $1<Q^{2}<75 G e V^{2} / c^{2}$. $R_{L}$ is implemented in the cross section as a correction to $F_{2}$ (discussed below).

\subsubsection{DIS Differential Cross Section}

The DIS cross section is implemented in the Monte Carlo in terms of the PDFs. The quark parton DIS cross section for neutrino and anti-neutrino scattering off a proton is given by

$$
\begin{aligned}
\frac{d^{2} \sigma_{0}\left(\nu_{\mu} p \rightarrow \mu^{-} X\right)}{d x d y} & =\frac{2 G_{F}^{2} M E}{\pi} \\
& \times\left[\left|V_{u d}\right|^{2} x d(x)+(1-y)^{2}\left(\left|V_{u s}\right|^{2}+\left|V_{u d}\right|^{2}\right) x \bar{u}(x)+2\left|V_{u s}\right|^{2} x s(x)\right] \\
\frac{d^{2} \sigma_{0}\left(\bar{\nu}_{\mu} p \rightarrow \mu^{+} X\right)}{d x d y} & =\frac{2 G_{F}^{2} M E}{\pi} \\
& \times\left[\left|V_{u d}\right|^{2} x \bar{d}(x)+(1-y)^{2}\left(\left|V_{u s}\right|^{2}+\left|V_{u d}\right|^{2}\right) x u(x)+2\left|V_{u s}\right|^{2} x \bar{s}(x)\right]
\end{aligned}
$$

where quark mixing is taken into account with the Cabbibo-Kobayashi-Maskawa (CKM) matrix elements $V_{i j}[19]$.

The longitudinal cross section and the target mass term $\frac{M x y}{2 E}$ are given by

$$
\begin{aligned}
\frac{d^{2} \sigma_{L}\left(\nu_{\mu} p \rightarrow \mu^{-} X\right)}{d x d y} & =\frac{2 G_{F}^{2} M E}{\pi}\left[\left(1-y-\frac{M x y}{2 E}\right) \cdot \frac{1+R_{L}\left(x, Q^{2}\right)}{1+4 M^{2} x^{2} / Q^{2}}+y-1\right] \\
& \times\left(\left|V_{u d}\right|^{2} x d(x)+\left(\left|V_{u s}\right|^{2}+\left|V_{u d}\right|^{2}\right) x \bar{u}(x)+2\left|V_{u s}\right|^{2} x s(x)\right) \\
\frac{d^{2} \sigma_{L}\left(\bar{\nu}_{\mu} p \rightarrow \mu^{+} X\right)}{d x d y} & =\frac{2 G_{F}^{2} M E}{\pi}\left[\left(1-y-\frac{M x y}{2 E}\right) \cdot \frac{1+R_{L}\left(x, Q^{2}\right)}{1+4 M^{2} x^{2} / Q^{2}}+y-1\right] \\
& \cdot\left[\left|V_{u d}\right|^{2} x \bar{d}(x)+\left(\left|V_{u s}\right|^{2}+\left|V_{u d}\right|^{2}\right) x u(x)+2\left|V_{u s}\right|^{2} x \bar{s}(x)\right] .
\end{aligned}
$$


We can obtain the scattering off a neutron by invoking the isospin symmetry $d \rightarrow u$ and $\bar{u} \rightarrow \bar{d}$. The total DIS cross section for a light quark isoscalar target is

$$
\begin{aligned}
\frac{d^{2} \sigma_{C C}^{\nu N}}{d x d y} & =\frac{1}{2}\left[\frac{d^{2} \sigma_{0}^{\nu p}}{d x d y}+\frac{d^{2} \sigma_{L}^{\nu p}}{d x d y}+\frac{d^{2} \sigma_{0}^{\nu n}}{d x d y}+\frac{d^{2} \sigma_{L}^{\nu n}}{d x d y}\right] \\
\frac{d^{2} \sigma_{C C}^{\bar{\nu} N}}{d x d y} & =\frac{1}{2}\left[\frac{d^{2} \sigma_{0}^{\bar{\nu} p}}{d x d y}+\frac{d^{2} \sigma_{L}^{\bar{\nu} p}}{d x d y}+\frac{d^{2} \sigma_{0}^{\bar{\nu} n}}{d x d y}+\frac{d^{2} \sigma_{L}^{\bar{\nu} n}}{d x d y}\right] .
\end{aligned}
$$

The remaining pieces of the cross section (including charm production which is not included in Equations 4.14 and 4.15) are described below.

4.1.3.1 Charm Production Cross Section When a neutrino scatters off a $d$ or $s$ quark it can produce a $c$ quark in the final state. Because $m_{c}$ is not small compared to $Q^{2}$ the Bjorken scaling variable $\mathrm{x}$ can not be used to describe the fractional momentum carried by the struck quark. From conservation of four momentum we have

$$
\begin{aligned}
(q+\xi p)^{2}=p^{\prime 2} & =m_{c}^{2} \\
q^{2}+2 \xi p \cdot q+\xi^{2} M^{2} & =m_{c}^{2}
\end{aligned}
$$

where $\xi$ is the momentum fraction carried by the struck quark, and $m_{c}$ is the charm mass. Neglecting the term $\xi^{2} M^{2}$ and $\frac{-q^{2}}{2 M \nu}=\frac{Q^{2}}{2 M \nu}=x$ we get

$$
x \rightarrow \xi=x \cdot\left(1+\frac{m_{c}^{2}}{Q^{2}}\right) .
$$

For the case of a massive quark in the final state the modified Bjorken scaling variable $\xi$ is used. In addition, due to the massive quark in the final state, kinematic production thresholds must be applied. This leads to a suppression of the charm production cross section.

This is the LO "slow rescaling" model obtained in references [13] and [29]. The structure functions are modified to include the massive charm quark effect by rescaling the $x \rightarrow \xi$ variable,

$$
\begin{aligned}
2 x F_{1}\left(x, Q^{2}\right) & \rightarrow \frac{x}{\xi} 2 \xi F_{1}\left(\xi, Q^{2}\right), \\
F_{2}\left(x, Q^{2}\right) & \rightarrow F_{2}\left(\xi, Q^{2}\right), \\
x F_{3}\left(x, Q^{2}\right) & \rightarrow \frac{x}{\xi} \xi F_{3}\left(\xi, Q^{2}\right),
\end{aligned}
$$




$$
F_{2}\left(\xi, Q^{2}\right)=\frac{1+R\left(\xi, Q^{2}\right)}{1+4 M^{2} \xi^{2} / Q^{2}} 2 \xi F_{1}\left(\xi, Q^{2}\right)
$$

where $R\left(\xi, Q^{2}\right)$ is the structure function $R_{L}$.

Substituting the structure functions in the DIS cross section we obtain the charm producing terms in the cross section

$$
\begin{aligned}
\frac{d^{2} \sigma\left(\nu_{\mu} N \rightarrow \mu^{-} c X\right)}{d x d y}=\frac{G_{F}^{2} M E_{\nu}}{\pi} & {\left[\left(\frac{y^{2}}{2} \frac{x}{\xi}+\frac{1+R\left(\xi, Q^{2}\right)}{1+4 M^{2} \xi^{2} / Q^{2}}\left(1-y-\frac{M x y}{2 E}\right)\right) 2 \xi F_{1}\left(\xi, Q^{2}\right)\right.} \\
\pm & \left.\left(y-\frac{y^{2}}{2}\right) \frac{x}{\xi} \xi x F_{3}\left(\xi, Q^{2}\right)\right]
\end{aligned}
$$

This can be rewritten in terms of the PDFs

$$
\begin{aligned}
\frac{d^{2} \sigma\left(\nu_{\mu} N \rightarrow \mu^{-} c X\right)}{d x d y}= & \frac{2 G_{F}^{2} M E}{\pi}\left[\frac{x y}{\xi}+\left(1-y-\frac{M \xi y}{2 E}\right) \cdot \frac{1+R_{L}\left(\xi, Q^{2}\right)}{1+4 M^{2} \xi^{2} / Q^{2}}\right] \\
\cdot \xi & {\left[\left|V_{c d}\right|^{2} \Theta\left(W-M_{\Lambda_{C}}\right)\left(d_{v}(\xi)+u_{v}(\xi)\right)\right.} \\
& +\left|V_{c d}\right|^{2} \Theta\left(W-M_{\Lambda_{C}}-M_{\pi}\right)(\bar{d}(\xi)+\bar{u}(\xi)) \\
& \left.+\left|V_{c s}\right|^{2} \Theta\left(W-M_{\Lambda_{C}}-M_{K}\right) s(\xi)\right],
\end{aligned}
$$

where the thresholds require the invariant mass of the final state, $W^{2}$, to be large enough to produce charmed mesons and baryons. Anti-neutrino DIS scattering can produce a $\bar{c}$ quark in the final state when it scatters off sea components $\bar{d}$ or $\bar{s}$. Similar to the neutrino case, the charm production cross section for anti-neutrino scattering off an isoscaler target is given by

$$
\begin{aligned}
\frac{d^{2} \sigma\left(\bar{\nu}_{\mu} N \rightarrow \mu^{-} c X\right)}{d x d y}= & \frac{2 G_{F}^{2} M E}{\pi}\left[\frac{x y}{\xi}+\left(1-y-\frac{M \xi y}{2 E}\right) \cdot \frac{1+R_{L}\left(\xi, Q^{2}\right)}{1+4 M^{2} \xi^{2} / Q^{2}}\right] \\
\cdot \xi & {\left[\left|V_{c d}\right|^{2} \Theta\left(W-M_{p}-M_{D}-M_{\pi}\right)(\bar{d}(\xi)+\bar{u}(\xi))\right.} \\
& \left.+\left|V_{c s}\right|^{2} \Theta\left(W-M_{p}-M_{D}-M_{K}\right) \bar{s}(\xi)\right] .
\end{aligned}
$$

If we neglect the target mass and the longitudinal terms, the charm suppression factor is given by the expression

$$
1-y+\frac{x y}{\xi}=1-\frac{m_{c}^{2}}{2 M E \xi} .
$$


There are several bounds to ensure the charm production is kinematically possible. We require $\xi \leq 1$ which can be also translated into requirements on the variables $x$ and $y$

$$
\begin{aligned}
\xi & =x \cdot\left(1+\frac{m_{c}^{2}}{Q^{2}}\right)=x+\frac{m_{c}^{2}}{2 M E y} \leq 1, \\
x & \leq 1-\frac{m_{c}^{2}}{2 M E y} \leq 1-\frac{m_{c}^{2}}{2 M E}, \\
y & \geq \frac{m_{c}^{2}}{2 M E(1-x)} \geq \frac{m_{c}^{2}}{2 M E} .
\end{aligned}
$$

The rescaling variable is bounded from below because $x>0$. The bound for $\xi$ is

$$
\xi \geq \frac{m_{c}^{2}}{2 M y E} \geq \frac{m_{c}^{2}}{2 M E}
$$

which implies that to probe small $\xi$, where the PDFs are large, the energy $E$ should be large enough to surpass this bound.

The slow rescaling model depends on the charm mass parameter. The value of the charm mass parameter used in our model is $m_{c}=1.40 \pm 0.18$, obtained from the weighted average of leading-order experimental measurements [20],[30]. The variance is taken to be the uncertainty on the charm mass value.

4.1.3.2 Non-Isoscalar Target Cross Section The NuTeV target is iron which contains an excess of neutrons

$$
\frac{N-Z}{A}=0.0567
$$

As a result there are more $d$-quarks than $u$-quarks in our target. If we take into account isospin symmetry $u^{n}=d^{p}$ and $u^{p}=d^{n}$, there is about a $2 \%$ excess of $d$-quarks over $u$ quarks. Since neutrino CC scattering is sensitive to flavor the cross section for neutrinos will be larger on iron than for a true isoscalar target, while the cross section for anti-neutrinos will be smaller. To account for this NUMONTE computes the cross section for protons and neutrons separately. The weighted average of the proton and neutron cross section gives the cross section for a non-isoscalar target

$$
\frac{d^{2} \sigma_{C C}^{\nu(\bar{\nu}) N}}{d x d y}=\left[\frac{Z}{A} \frac{d^{2} \sigma_{C C}^{\nu(\bar{\nu}) p}}{d x d y}+\frac{N}{A} \frac{d^{2} \sigma_{C C}^{\nu(\bar{\nu}) n}}{d x d y}\right] .
$$



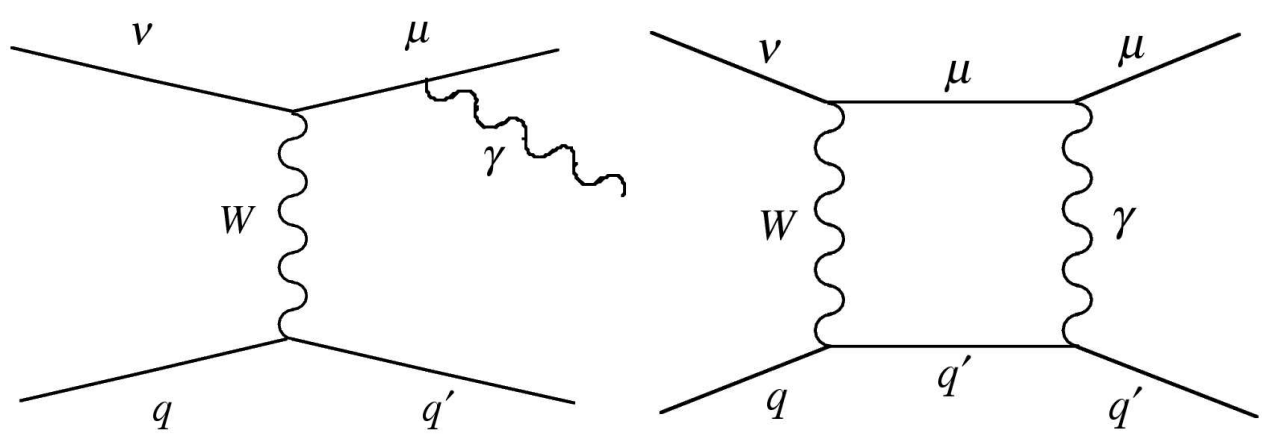

Figure 4.1: Diagrams for QED radiative corrections for CC neutrino scattering.

4.1.3.3 Propagator Term A neutrino interacts with matter via the weak interaction, which for neutrino DIS CC process is mediated by the massive virtual W boson. The effect of the massive virtual boson is contained in the propagator term

$$
\frac{d^{2} \sigma_{C C}^{\nu(\bar{\nu})}}{d x d y}=\frac{1}{\left(1+Q^{2} / M_{W}^{2}\right)^{2}} \cdot \frac{d^{2} \sigma_{C C}^{\nu(\bar{\nu})}}{d x d y}
$$

\subsubsection{Radiative Corrections}

Radiative corrections are applied to the "Born"-level differential cross section (Equation 1.18) using Bardin's calculation [31] to account for contributions to the cross section due to real and virtual photon radiation by the charged particles. Figure 4.1 shows two of the diagrams included in Bardin's calculation. Photon radiation by the charged lepton in the final state has the largest contribution.

Radiative corrections should be applied to each event, but recomputing them for each event is slow and inefficient. Instead, a table is generated for a number of $\mathrm{x}, \mathrm{y}, \mathrm{E}$ grid points which contains the 1-loop QED radiative correction for CC neutrino interactions

$$
C^{R A D}=\left[\left(\frac{d^{2} \sigma_{C C}}{d x d y}\right)_{Q E D 1-l o o p} /\left(\frac{d^{2} \sigma_{C C}}{d x d y}\right)_{\text {Born }}\right]_{\text {Bardin }}
$$

for each point of the grid. This table is used to obtain radiative corrections for any $\mathrm{x}, \mathrm{y}, \mathrm{E}$ by interpolating between the grid points. Radiative corrections are applied as a multiplicative 
correction to the "Born"-level CC differential cross section

$$
\frac{d^{2} \sigma_{C C}}{d x d y}=C^{R A D}\left(\frac{d^{2} \sigma_{C C}}{d x d y}\right)_{B o r n} .
$$

Figures 4.2-4.4 show the 1-loop QED radiative corrections for charged current neutrino (red solid curves) and anti-neutrino (blue dashed curves) interactions for energies $E_{\nu}=45$ $\mathrm{GeV}, E_{\nu}=95 \mathrm{GeV}$ and $E_{\nu}=245 \mathrm{GeV}$ respectively. The kinks at low x and low y are due to the transition between our BG parameterization and GRV94 extrapolation.

\subsubsection{Cross Section Fit}

An empirical fit to the extracted cross section table (see Chapter 6) is used to determine the PDF parameters. The fit includes $\mathrm{NuTeV}$ data in the kinematic region, $0.001<x<0.7$, $30<E<360 \mathrm{GeV}$, and $Q^{2}>1.35 \mathrm{GeV}^{2} / \mathrm{c}^{2}$. In order to constrain the fit at high $x$ and low $Q^{2}$, we use charge lepton, $F_{2}^{l}$, proton and deuterium data from SLAC [25], BCDMS [26] and NMC [27] for $x>0.4 . F_{2}^{l}$ is converted to $F_{2}^{\nu}$ using the 5/18th rule (Equation 1.41), using CTEQ4D PDFs [23]. For the nuclear target correction we use an $x$ dependent parameterization of $F_{2}^{F e} / F_{2}^{d}$ data from charge lepton DIS obtained in reference [32]

$$
f(x)=1.10-0.36 x-0.28 e^{-21.9 x}+2.77 x^{14.4} .
$$

Since nuclear effects are not measured for neutrino scattering the standard treatment is to assume that the nuclear correction is the same for neutrino scattering as for charge lepton scattering. As a result, the $x$ dependence of charge lepton data may differ from the $x$ dependence of our data. Therefore, relative normalizations are introduced as parameters in the fit to make sure the cross section fit follows our data. The fit is performed by minimizing the $\chi^{2}$ given by

$$
\chi^{2}=\chi_{N u T e V}^{2}+\chi_{S L A C}^{2}+\chi_{B C D M S}^{2}+\chi_{N M C}^{2},
$$

where each individual $\chi^{2}$ was computed using

$$
\chi^{2}=\sum_{i=1}^{N^{D A T A}} \frac{\left(D A T A_{i}-B G_{i}\right)^{2}}{\sigma_{i}^{2}} .
$$




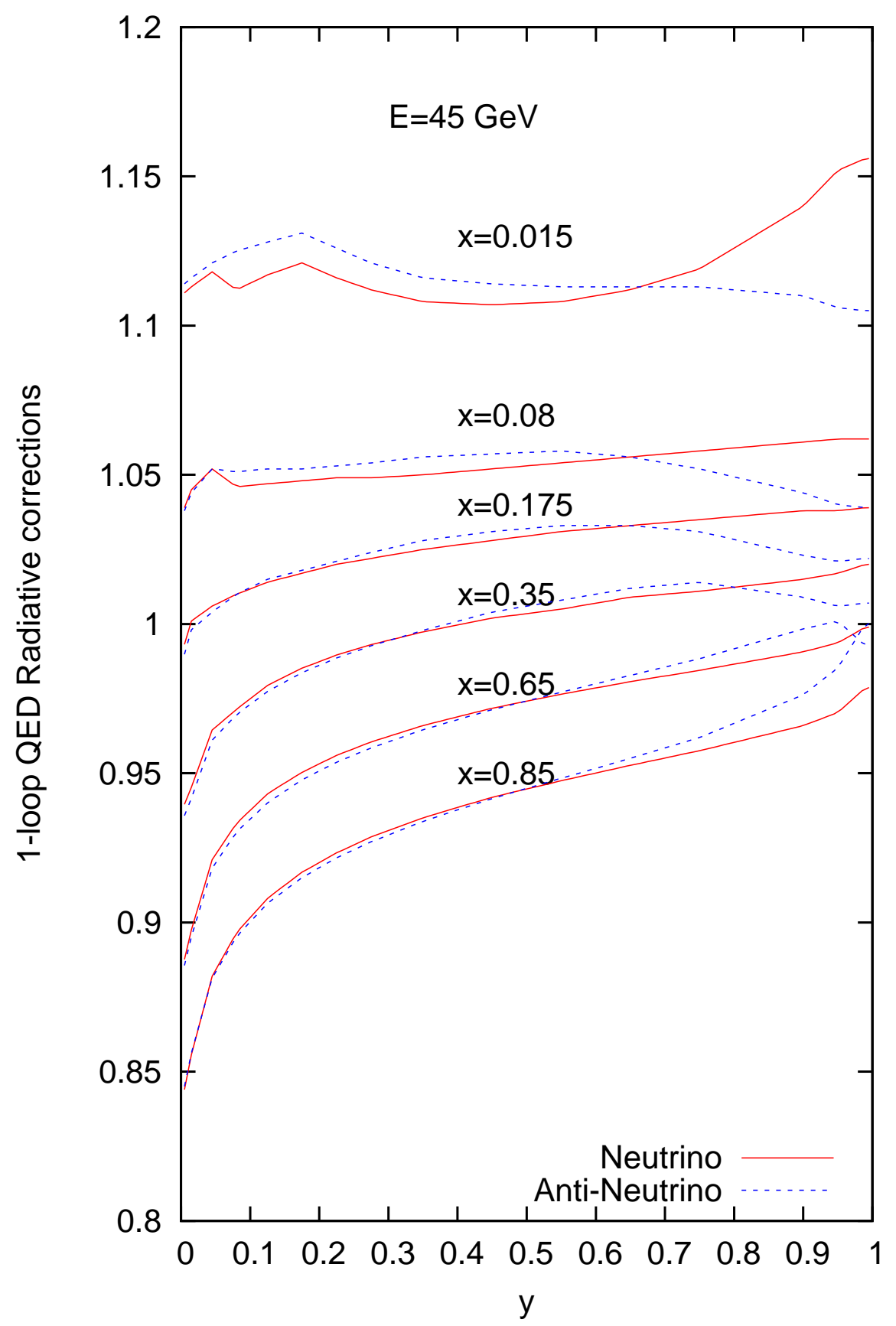

Figure 4.2: 1-loop QED radiative corrections for charged current neutrino (red solid curve) and anti-neutrino (blue dashed curve) interactions for incident energy $\mathrm{E}=45 \mathrm{GeV}$ for various $\mathrm{x}$ values. 


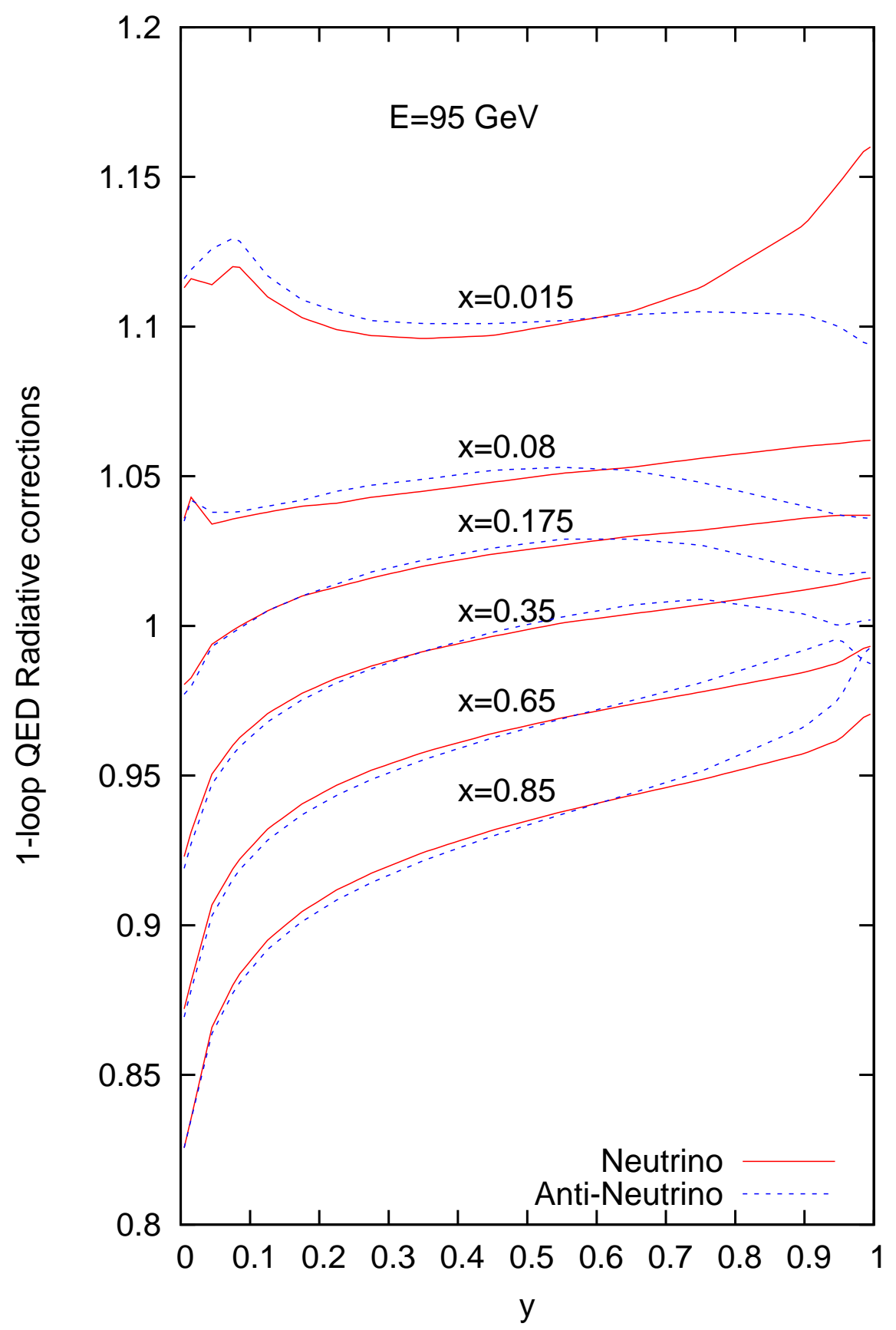

Figure 4.3: 1-loop QED radiative corrections for charged current neutrino (red solid curve) and anti-neutrino (blue dashed curve) interactions for incident energy $\mathrm{E}=95 \mathrm{GeV}$ for various $\mathrm{x}$ values. 


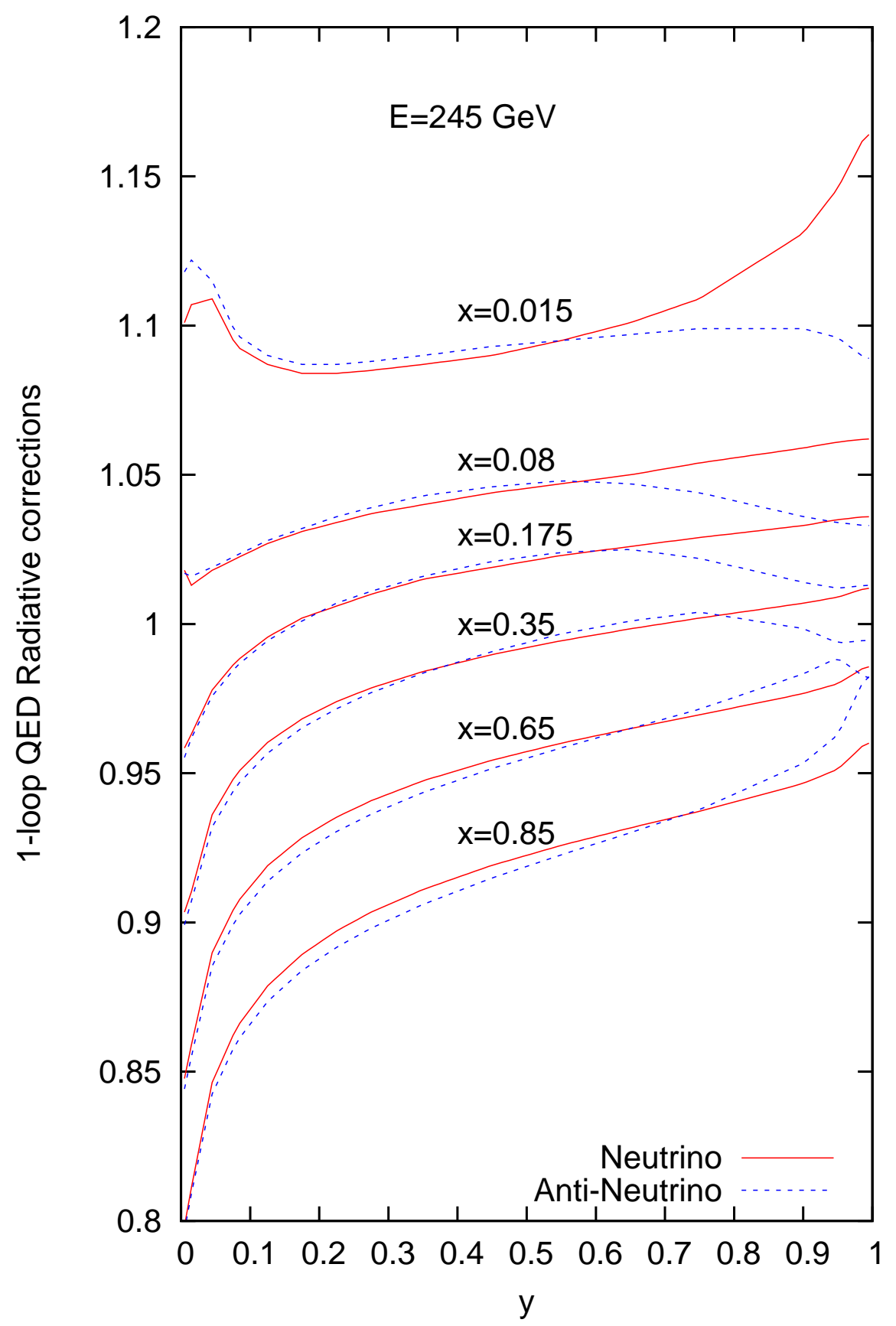

Figure 4.4: 1-loop QED radiative corrections for charged current neutrino (red solid curve) and anti-neutrino (blue dashed curve) interactions for incident energy $\mathrm{E}=245 \mathrm{GeV}$ for various $\mathrm{x}$ values. 
$D A T A_{i}$ are the cross section points for the $\mathrm{NuTeV}$ data, and $F_{2}$ for SLAC, BCDMS and NMC data. $B G_{i}$ represents the cross section model or $F_{2}$ calculated using our BG model.

The values of the 19 free PDF parameters and their uncorrelated errors, obtained from the fit to the final cross section table, are given in Table 4.1. Figure 4.5 shows the parton densities computed with the final PDF parameters at $Q_{0}^{2}=12.6 \mathrm{GeV}$.

\subsection{EVENT GENERATION}

Event generation starts by setting the type of the beam and the corresponding muon spectrometer polarity. The 'TRUTH' variables or "GENERATED" variables are denoted with a ' $G$ ' at the end of the variable's name. The following procedure is executed to generate charged-current neutrino(anti-neutrino) interaction events:

- Incident neutrino energy - The incident neutrino energy "ENUG" is randomly selected by sampling the neutrino flux spectrum, $\Phi(E)$. The neutrino flux is measured from data (see Chapter 5).

- Transverse vertex- The transverse position of the neutrino interaction (VXG, VYG) for a given energy, ENUG, is selected randomly by sampling (in 2-D) the transverse vertex distribution for this energy which is found from the data.

- Longitudinal vertex - The longitudinal interaction position PLACEG is randomly selected from a uniform distribution. In each steel plate VZG (the $z$ vertex location) is generated between the center of counter PLACEG and PLACEG+1 (one counter upstream from PLACEG) according to the mass distribution in the detector.

- Bjorken x and Inelasticity - Both TRUTH kinematic variable, XG and YG, are generated by sampling the shape of the differential cross section given by $\frac{d^{2} \sigma}{E d x d y}(E N U G, X G, Y G)$.

- Kinematic Variables - All remaining kinematic variables can be computed from XG, YG, and ENUG using Equations 1.3-1.8. These include EHADG, the TRUTH hadron energy, THETAMUG the TRUTH muon angle at the vertex, EMUG, the TRUTH muon energy, QSQG, the TRUTH four momentum transfer. The TRUTH angle, PHIMUG, is generated from a uniform distribution between 0 and $2 \pi$. 
Table 4.1: The final parameters for the best BGPAR fit.

\begin{tabular}{|l|l|r|r|}
\hline Parameter & Description & Value & Uncertainty \\
\hline$A_{0}$ & Lambda & 0.583 & 0.017 \\
$A_{1}$ & LO Valence Normalization & 0.295 & 0.013 \\
$A_{2}$ & NLO Valence Normalization & 0.17 & 0.03 \\
$E_{10}$ & 1st Valence term $x$ exponent at $Q_{0}^{2}$ & 0.5333 & 0.0025 \\
$E_{11}$ & Valence $x$ exponents' slope in $Q^{2}$ & -0.028 & 0.011 \\
$E_{20}$ & 1st Valence term $(1-x)$ exponent at $Q_{0}^{2}$ & 2.61 & 0.015 \\
$E_{21}$ & Valence $(1-x)$ exponents' slope in $Q^{2}$ & 1.31 & 0.045 \\
$A V_{2}$ & 2nd Valence term constant & 637 & 75.0 \\
$E_{30}$ & 2nd Valence term $x$ exponent at $Q_{0}^{2}$ & 4.56 & 0.14 \\
$E_{40}$ & 2nd Valence term $(1-x)$ exponent at $Q_{0}^{2}$ & 12.5 & 0.35 \\
$S_{2}$ & Total Sea 2 nd moment in $x$ at $Q_{0}^{2}$ & 0.1625 & 0.0013 \\
$S_{3}$ & Total Sea 3rd moment in $x$ at $Q_{0}^{2}$ & 0.01589 & 0.0004 \\
$G_{3}$ & Gluon 3rd moment in $x$ at $Q_{0}^{2}$ & 0.031 & 0.003 \\
$A S_{20}$ & 2nd SEA term constant at $Q_{0}^{2}$ & 1.06 & 0.11 \\
$A S_{21}$ & 2nd SEA term constant slope in $Q^{2}$ & 1.76 & 0.25 \\
$E S_{20}$ & 2nd SEA term $(1-x)$ exponent at $Q_{0}^{2}$ & 0.3332 \\
$E S_{21}$ & 2nd SEA term $(1-x)$ exponent slope in $Q^{2}$ & 8.4 & 0.02 \\
\hline$A_{H T}$ & Higher twist model parameter A & 2.5 \\
$B_{H T}$ & strange sea normalization & \\
\hline$\kappa$ & strange sea shape & \\
$\alpha$ & & & \\
\hline
\end{tabular}




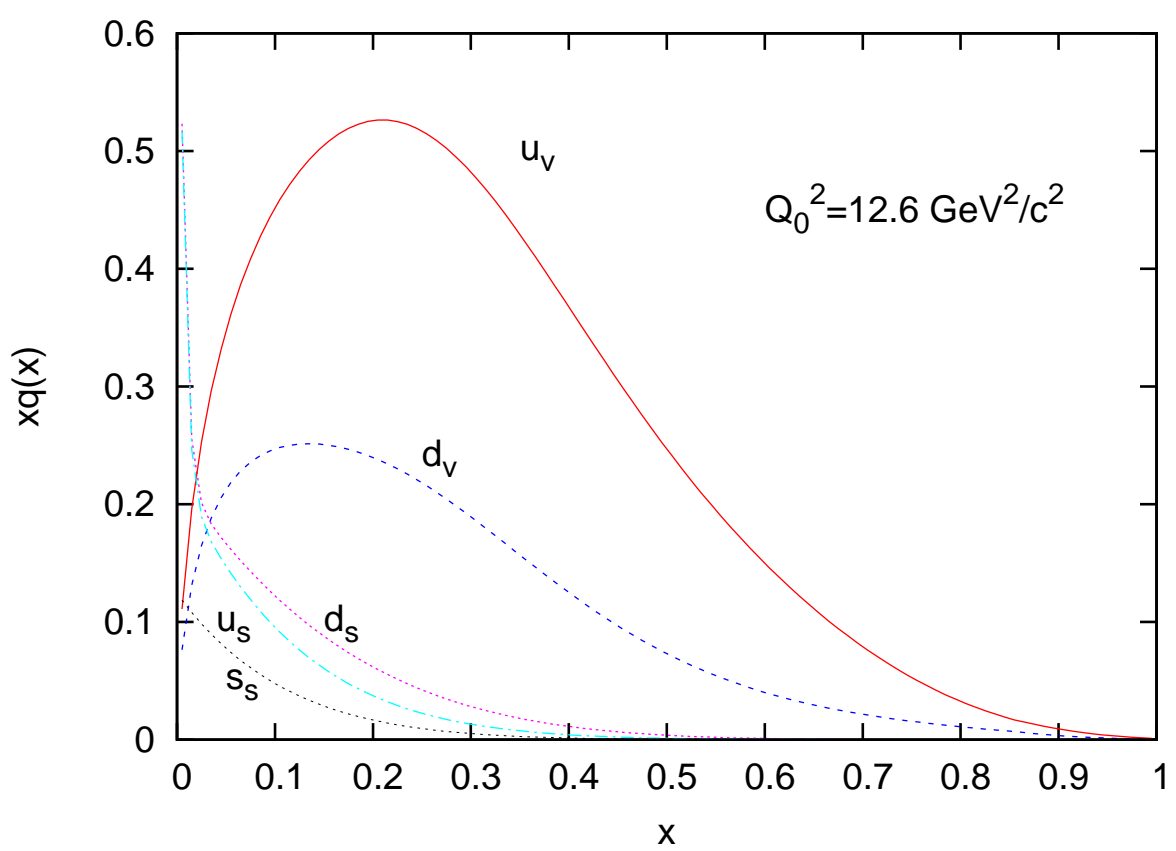

Figure 4.5: Quark distributions calculated using our final BG model parameters at the initial $Q_{0}^{2}=12.6 \mathrm{GeV}$.

- Muon energy loss - A muon track at initial angle THETAMUG and energy EMUG is stepped through the target calorimeter and its energy loss and MCS are simulated for each step. The new muon energy after each step is obtained by subtracting the energy loss in the upstream steps. Energy loss for muons less than $1 \mathrm{GeV}$ is taken from rangeenergy tables. For muons with energy greater than $1 \mathrm{GeV}$, energy loss is simulated by including ionization loss, pair production, $\delta$-ray production and bremsstrahlung. NUMONTE keeps track of the restricted energy loss, RESLOS, and the catastrophic energy loss, CATLOS, for each step. If the muon stops in the target the last counter is marked as CEXITG. When the muon reaches the end of the target calorimeter the total energy loss $D E D X G=R E S L O S+C A T L O S$ is subtracted from the muon energy at the vertex, EMUG. This gives the TRUTH energy of the muon at the front face $(\mathrm{FF})$ of the toroid $E M U F F G=E M U G-D E D X G$. The TRUTH position of the muon at the front face of the toroid given by, $V X F F G$ and $V Y F F G$, also results from the stepping procedure. 
- Hadron shower length - Hadron energy dependent longitudinal shower distributions are determined from hadron test beam data. The resulting library of hadron showers are used to generate SHEND. The hadron shower length for the charge-current analysis is defined as (PLACE - SHEND + 5).

\subsection{DETECTOR MODEL}

In order to describe data the Monte Carlo simulation must model the response of the detector to muon and hadron interactions in the detector. NUMONTE smears the TRUTH kinematic variables to simulate detector resolution effects by using a parameterization of a test beam data in some cases, when possible the response is simulated with a hit-level GEANT based Monte Carlo $(\mathrm{McNuTeV})$. All physics quantities are then reconstructed using the same procedure as quantities in real data. For the charged-current analysis EMUG, EHADG, THETAG, PHIG, EMUFFG, PLACEG and SHENDG are smeared to simulate the detector response. The corresponding smeared variables are EMU, EHAD, THETA, PHI, EMUFF, PLACE and SHEND. The resolution for measuring the muon track vertex position is small enough (less than $0.25 \mathrm{~cm}$ ) that the smearing of the transverse vertex position variables (VXG, VYG) are neglected for the charged-current analysis.

\subsubsection{Muon Energy Smearing and Reconstruction}

As described in the previous section the muon is projected through the target and NUMONTE keeps track of both restricted and catastrophic energy loss. Restricted energy loss, RESLOS, is smeared as a Gaussian with a width of $0.045 \mathrm{GeV}$. Catastrophic energy loss, CATLOS, is smeared according to the resolution function for electromagnetic showers obtained from electron test beam data (Section 3.1). The smearing function for CATLOS is a Gaussian having width $0.5 \sqrt{C A T L O S}$. A pulseheight in MIPS is calculated for each counter from the smeared CATLOS and RESLOS as follows: RESLOS is converted to MIPS to give $E C T R^{R E S}(i)$ using the muon conversion factor, $C_{\mu}$. CATLOS is converted to MIPS to 
give $E C T R^{C A T}(i)$, using the conversion factor for electromagnetic showers, $C_{e}$. The total simulated pulseheight for each counter in MIPS is

$$
\operatorname{ECTR}(i)=\operatorname{ECTR} R^{R E S}(i)+\operatorname{ECTR}^{C A T}(i)
$$

The generated muon energy in the toroid EMUFFG is smeared by sampling the toroid resolution function

$$
R=\frac{1 / P M U F F-1 / P M U F F G}{1 / P M U F F G}
$$

where $P M U F F G=\sqrt{E M U F F G^{2}-m_{\mu}^{2}}$ and $P M U F F=\sqrt{E M U F F^{2}-m_{\mu}^{2}}$ are the generated and the smeared muon momenta and EMUFF is the smeared muon energy in the toroid. Equation 3.20 is used as the resolution function which is obtained by fitting the detector response to calibration beam muons with energies ranging from 30 to $170 \mathrm{GeV}$ (see Section 3.3.4).

4.3.1.1 Muon Energy Reconstruction The reconstruction of the muon energy is divided in three components:

- $P S H W R$ is the muon energy deposited in the shower region. It is computed as a sum of the average muon energy deposition in $\mathrm{GeV}$ in counters from PLACE to SHEND - 5 using Equation 3.14.

- PTARG is the muon energy deposition in the target beyond the shower region. It is computed as the sum of the pulseheights $\operatorname{ECTR}(i)$ from $S H E N D-6$ to 1 and converted to $\mathrm{GeV}$ using Equations 3.11-3.13.

- EMUFF is muon energy measured in the toroid.

The total muon energy is $E M U=P S H W R+P T A R G+E M U F F$. 


\subsubsection{Muon Angle Smearing}

The muon angular resolution depends on the muon momentum and length of the track in the target because it is dominated by MCS. A number of chambers downstream from vertex are omitted from the muon track depending on the shower energy therefore, the muon angle resolution will depend on the hadron energy. $\mathrm{McNuTeV}$ simulation is used to parameterize the angular resolution function

$$
\Delta \theta_{\mu}=(96.8+0.87 \text { PLACE }+0.24 E H A D G) / P M U G,
$$

where EHADG is the generated hadron energy, and $P M U G=\sqrt{E M U G^{2}-m_{\mu}^{2}}$ is the generate muon momentum.

\subsubsection{Hadron Energy Smearing and Reconstruction}

The generated hadron energy, EHADG, is calibrated to simulate the non-linearity of the $\mathrm{NuTeV}$ calorimeter (see Section 3.1)

$$
E H A D G=E H A D G \times \frac{e \cdot f_{\pi^{0}}(E H A D G)+h \cdot\left[1-f_{\pi^{0}}(E H A D G)\right]}{e \cdot f_{\pi^{0}}(75)+h \cdot\left[1-f_{\pi^{0}}(75)\right]} .
$$

EHADG is then smeared by sampling the Poisson-like resolution function given by Equation 3.9. The simulated pulseheight, $\operatorname{ECTR}(i)$, given by Equation 4.32 is added to account for the muon energy deposited in the shower region. To simulate the smeared hadron energy variable, $E H A D$, the muon energy loss is then subtracted back out from the hadron energy using the parameterization of the restricted energy loss, $E_{\mu}^{\text {loss }}(i)$, in MIPs given by equation 3.6. The smeared hadronic energy is given by

$$
E H A D=E H A D G^{\text {smeared }}+C_{h} \sum_{i=P L A C E}^{S H E N D-5}\left(E C T R(i)-E_{\mu}^{\text {loss }}(i)\right) \text {, }
$$

where $C_{h}=0.212 \mathrm{GeV} / \mathrm{MIP}$ is the hadron conversion factor.

The remaining smeared kinematic variables are computed from EMU,THETAMU, and EHAD using Equations 1.3-1.8. 


\subsection{NEUTRINO FLUX AND NEUTRINO TOTAL CROSS SECTION}

In principle, the absolute neutrino flux can be determined by monitoring the secondary particles in the beam, ( $\pi$ 's and $K$ 's), which decay to produce the beam neutrinos. Hence, we could obtain the absolute neutrino flux from 2-body decay kinematics applied to the

measured secondary fluxes. However, the high intensity of the beam and the broad-band energy spectrum makes it very difficult to accurately monitor these secondary particles. Instead, $\mathrm{NuTeV}$ measures "relative" neutrino flux from a sample of charged-current neutrino interactions. The fixed- $\nu_{0}[3,32]$ method is used to extract the energy spectrum of the neutrino and anti-neutrino fluxes, "relative flux", while the overall normalization is obtained by comparing the level of the $\mathrm{NuTeV}$ total neutrino cross section to existing absolute total cross section measurements.

\subsection{FLUX EXTRACTION METHOD}

The relative neutrino flux is extracted from a sample of charge current neutrino interactions with hadronic energy less than $\nu_{0}$. If we rewrite the DIS charge current differential cross section in terms of $\nu$, (where $\nu=E_{H A D}$ ), by substituting $y E=\nu$, we obtain

$$
{\frac{d^{2} \sigma^{\nu, \bar{\nu}}}{d x d \nu}}^{=} \frac{G^{2} M}{\pi}\left[\left(1-\frac{\nu}{E}-\frac{M x \nu}{2 E^{2}}+\frac{\nu^{2}}{2 E^{2}} \frac{1+2 M x / \nu}{1+R}\right) F_{2} \pm \frac{\nu}{E}\left(1-\frac{\nu}{2 E}\right) x F_{3}\right] .
$$

Integrating over all $x$ for fixed $\nu$ gives:

$$
\frac{d \sigma^{\nu, \bar{\nu}}}{d \nu}=\frac{G^{2} M}{\pi}\left(\int F_{2} d x\right)\left[1-\frac{\nu}{E}\left(1 \mp \frac{\int x F_{3}}{\int F_{2}}\right)+\frac{\nu^{2}}{2 E^{2}}\left(1 \mp \frac{\int x F_{3}}{\int F_{2}}+\frac{\int F_{2} R_{T E R M}}{\int F_{2}}\right)\right]
$$


where

$$
R_{T E R M}=\left(\frac{1+\frac{2 M x}{\nu}}{1+R_{L}(x)}-\frac{M x}{\nu}-1\right)
$$

Multiplying both sides by the flux, $\Phi(E)$, will give the number of events in $\nu$-bins

$$
\frac{d N}{d \nu}=\Phi(E) A\left(1+\frac{B}{A} \frac{\nu}{E}-\frac{C}{A} \frac{\nu^{2}}{2 E^{2}}\right)
$$

where

$$
\begin{aligned}
A & =\frac{G_{F} M}{\pi} \int F_{2}(x) d x \\
B & =-\frac{G_{F} M}{\pi} \int\left(F_{2}(x) \mp x F_{3}(x)\right) \\
C & =B-\frac{G_{F} M}{\pi} \int F_{2}(x) R_{\text {TERM }} d x
\end{aligned}
$$

If we take the limit $\nu \rightarrow 0$, the number of events are directly proportional to the flux with a common constant of proportionality, $A$.

$$
\frac{d N}{d \nu}=\Phi(E) A\left(1+\frac{B}{A} \frac{\nu}{E}-\frac{C}{A} \frac{\nu^{2}}{2 E^{2}}\right) \rightarrow \Phi(E) A
$$

Substituting for $C$ we get

$$
\frac{d N}{d \nu}=\Phi(E) A\left(1+\frac{B}{A}\left(\frac{\nu}{E}-\frac{\nu^{2}}{2 E^{2}}\right)+\frac{\nu^{2}}{2 E^{2}} \frac{\int F_{2} R_{T E R M}}{\int F_{2}}\right),
$$

where $\frac{B}{A}=-\left(1 \pm \frac{\int x F_{3}}{\int F_{2}}\right)$. The structure functions depend on $x$ and $Q^{2}$, hence the integrals of the structure functions over $x$ are independent of energy. However, the scaling violations introduce an implicit small $\nu$ dependence. When we integrate $F_{2}$ and $x F_{3}$ over all $x$ at fixed $\nu$ the integral is performed over the values of $Q^{2}$ given by $\left(x, Q^{2}=2 x M \nu\right)$. For different fixed $\nu$ we integrate along a different line in the $\left(x, Q^{2}\right)$ space. Figure 5.1 shows the $\nu$-dependence of $\frac{B}{A}$, obtained by computing the integrals $\int_{0}^{1} x F_{3} d x$ and $\int_{0}^{1} F_{2} d x$, using two different PDF parameterizations - our BG model and the model from reference [33].

Integration of Equation 5.6 over $\nu$ in the interval $\left(0, \nu_{0}\right)$ gives the number of events, which are directly proportional to the flux

$$
N(E)_{\nu<\nu_{0}}=\Phi(E) \int_{0}^{\nu_{0}} d \nu A\left[1+\frac{B}{A}\left(\frac{\nu}{E}-\frac{\nu^{2}}{2 E^{2}}\right)+\frac{\nu^{2}}{2 E^{2}} \frac{\int F_{2} R_{T E R M}}{\int F_{2}}\right] .
$$



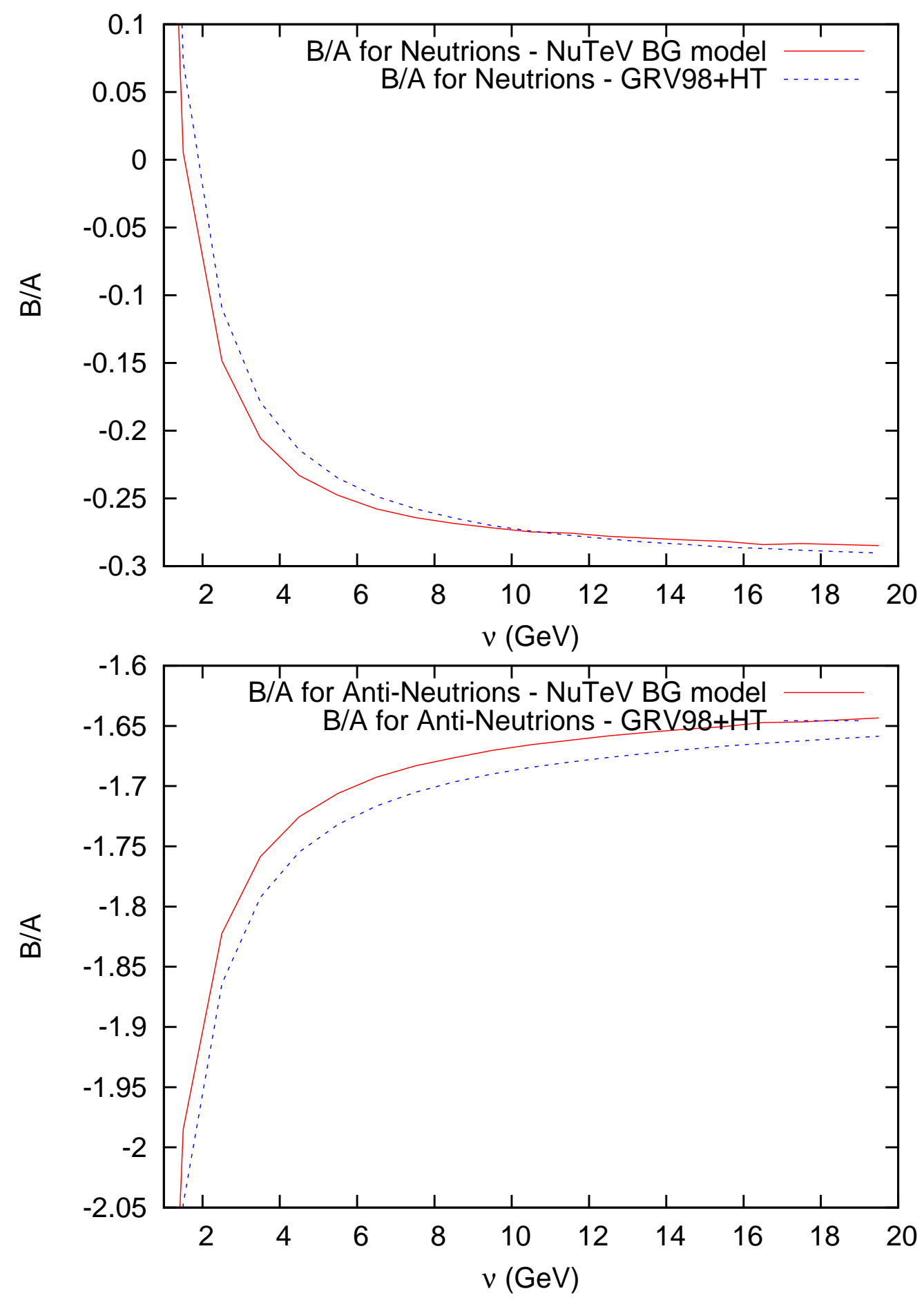

Figure 5.1: B/A for neutrinos (top) and anti-neutrinos (bottom) calculated with two different PDF sets. NuTeV BG model (red solid line) and GRV98 with Higher Twist (blue dashed line). 
The integral over $\nu$ has a small energy dependence, which becomes important at low neutrino energy. Since the proportionality constant is the same for any energy, Equation 5.7 can be used to determine the energy dependence of the neutrino flux (relative flux). The method of extracting the flux from our data sample is described in the next section.

\subsection{FLUX EXTRACTION}

The neutrino flux is extracted from a flux sample which is defined in Section 3.4. To apply the fixed- $\nu_{0}$ method the choice of $\nu_{0}$ optimizes the following two requirements: 1) high statistics is needed to determine the flux accurately, which is achieved by making $\nu_{0}$ as large as possible, and 2) the need to reduce the correlation between the flux and the structure functions samples, which requires that $\nu_{0}$ not be too large thereby minimizing the sample overlap.

The total number of events passing the flux cuts is 426803 for neutrinos and 210795 for anti-neutrinos. The events are binned in 17 bins of neutrino energy. The $E_{\nu}$ bin size is chosen to reflect that the number of events and the energy resolution vary with energy. Table 5.1 shows the number of raw events in energy bins for neutrinos and anti-neutrinos. In addition, the events in each energy bin $E_{\nu}$ are divided into $20 \nu$ bins with bin size of $1 \mathrm{GeV}$. The size of the $\nu$ bins is limited by statistics. The number of events binned in each $(\nu)$ bin represents $\frac{d N}{d \nu}$ for a given $E_{\nu}$. Using equation 5.7 the relative flux is

$$
\Phi(E) \propto \frac{\int_{0}^{\nu_{0}} \frac{d N}{d \nu} d \nu}{\int_{0}^{\nu_{0}} d \nu\left[1+\frac{B}{A}\left(\frac{\nu}{E}-\frac{\nu^{2}}{2 E^{2}}\right)+\frac{\nu^{2}}{2 E^{2}} \frac{\int F_{2} R_{T E R M}}{\int F_{2}}\right]}
$$

where the numerator is a sum of the raw number of events, corrected for detector acceptance and smearing, and the denominator is the $\nu / E$ correction accounting for the energy dependence.

We use the NUMONTE simulation to correct the raw number of events $\frac{d N}{d \nu}^{D A T A_{R A W}}$ for detector acceptance and smearing in each bin. Monte Carlo samples are generated using as input our cross section model and an initial flux, and events are binned as in data. An 


\begin{tabular}{|c|c|c|c|}
\hline Energy bin & Bin limits & Num. of events $\nu$-mode & Num. of events $\bar{\nu}$-mode \\
\hline 1 & $30-40 \mathrm{GeV}$ & 41294 & 21498 \\
\hline 2 & $40-50 \mathrm{GeV}$ & 51746 & 26889 \\
\hline 3 & $50-60 \mathrm{GeV}$ & 53646 & 28571 \\
\hline 4 & $60-70 \mathrm{GeV}$ & 51946 & 27349 \\
\hline 5 & $70-80 \mathrm{GeV}$ & 43680 & 23070 \\
\hline 6 & $80-90 \mathrm{GeV}$ & 32829 & 17268 \\
\hline 7 & $90-100 \mathrm{GeV}$ & 22883 & 11625 \\
\hline 8 & $100-120 \mathrm{GeV}$ & 25992 & 12882 \\
\hline 9 & $120-140 \mathrm{GeV}$ & 14756 & 6742 \\
\hline 10 & $140-160 \mathrm{GeV}$ & 12924 & 5294 \\
\hline 11 & $160-180 \mathrm{GeV}$ & 13328 & 5000 \\
\hline 12 & $180-200 \mathrm{GeV}$ & 11852 & 4326 \\
\hline 13 & $200-230 \mathrm{GeV}$ & 13331 & 4291 \\
\hline 14 & $230-260 \mathrm{GeV}$ & 7910 & 2296 \\
\hline 15 & $260-290 \mathrm{GeV}$ & 3962 & 1096 \\
\hline 16 & $290-320 \mathrm{GeV}$ & 1990 & 461 \\
\hline 17 & $320-360 \mathrm{GeV}$ & 1063 & 241 \\
\hline
\end{tabular}

Table 5.1: Raw number of events in neutrino energy bins passing flux cuts for neutrinos and anti-neutrinos. 
acceptance correction, $A C_{i j}^{F L U X}$, is applied to data to account for detector acceptance and smearing, for each $E_{i}, \nu_{j}$ bin. The corrected data are then given by

$$
\frac{d N}{d \nu}_{i j}^{D A T A_{C O R}}=\frac{d N}{d \nu}_{i j}^{D A T A_{R A W}} \cdot A C_{i j}^{F L U X}=\frac{d N}{d \nu}_{i j}^{D A T A_{R A W}} \cdot \frac{\frac{d N}{d \nu} i j}{\frac{d N}{d \nu}_{i j} S M E_{F L U X}}
$$

where $\frac{d N}{d \nu}_{i j}^{G E N_{F L U X}}$, is the number of generated events in bin $i j$, and $\frac{d N}{d \nu}_{i j} M E_{F L U X}$, is the number of smeared events (the sum of events which stayed in and entered the bin after smearing) in the bin. Figure 5.2 shows a comparison of $\frac{d N}{d \nu}_{i j}^{D A T A}, \frac{d N}{d \nu}{ }_{i j} S M E_{F L U X}$ and $\frac{d N}{d \nu}_{i j} G E N_{F L U X}$ for neutrinos and anti-neutrinos respectively in a typical energy bin $\left(E_{\nu}=75 \mathrm{GeV}\right)$. NUMONTE models data well except for at lowest $\nu$. (The low- $\nu$ difference is discussed below.)

In order to calculate the $\nu / E$ correction (denominator in equation 5.8) we need to obtain the values of $\frac{\int F_{2} R_{T E R M}}{\int F_{2}}$ and $B / A$. The value of $\frac{\int F_{2} R_{T E R M}}{\int F_{2}}$ is estimated by performing the integrals explicitly using Equation 5.3. The structure function $F_{2}$ is computed from our PDFs (Section 4.1.5) and $R_{L}$ model given by Equation 4.14. Figure 5.3 shows the value of $\frac{\int F_{2} R_{T E R M}}{\int F_{2}}$ as function of $\nu$. This term depends only on $\nu$, so it is the same for all energies.

The value of $B / A$ used to compute the $\nu / E$ correction is obtained by fitting the $\nu$ dependence of the data to the functional form of Equation 5.4, which can be rewritten as

$$
\begin{aligned}
\frac{d N^{D A T A}}{d \nu} & =A A \cdot\left(1-\frac{\nu}{E}+\frac{\nu^{2}}{2 E^{2}}\left(1+\frac{\int F_{2} R_{T E R M}}{\int F_{2}}\right)\right) \\
& +B B \cdot\left(\frac{\nu}{E}-\frac{\nu^{2}}{2 E^{2}}\right) .
\end{aligned}
$$

$A A$ and $B B$ are the fit parameters, which in principle correspond to $\Phi(E) \int F_{2}$ and $\Phi(E) \int x F_{3}$. We can compute $B / A$ from $A A$ and $B B$ as $\frac{B}{A}=-1+\frac{B B}{A A}$. Since the flux is a common normalization factor it cancels in $B / A$. Equation 5.10 is derived from Equation 5.1, which is the bare DIS differential cross section. Therefore, before fitting, data must be corrected for radiative effects, charm mass, isoscalar target and propagator. These corrections are computed by integrating our cross section model within the kinematic limits for each $\left(\nu, E_{\nu}\right)$ bin

$$
C O R_{i j}^{\text {bare }}=\frac{\int_{0}^{1} d x \frac{d^{2} \sigma\left(i s o, m c=0, \text { NoRad.Corr }, M_{W}=0\right)}{d x d y}\left(x, \nu_{j} / E_{i}, E_{i}\right)}{\int_{0}^{1} d x \frac{d^{2} \sigma\left(F e, m c=1.4 G e V, R a d . C o r r, M_{W}=80.4 G e V\right)}{d x d y}\left(x, \nu_{j} / E_{i}, E_{i}\right)}
$$



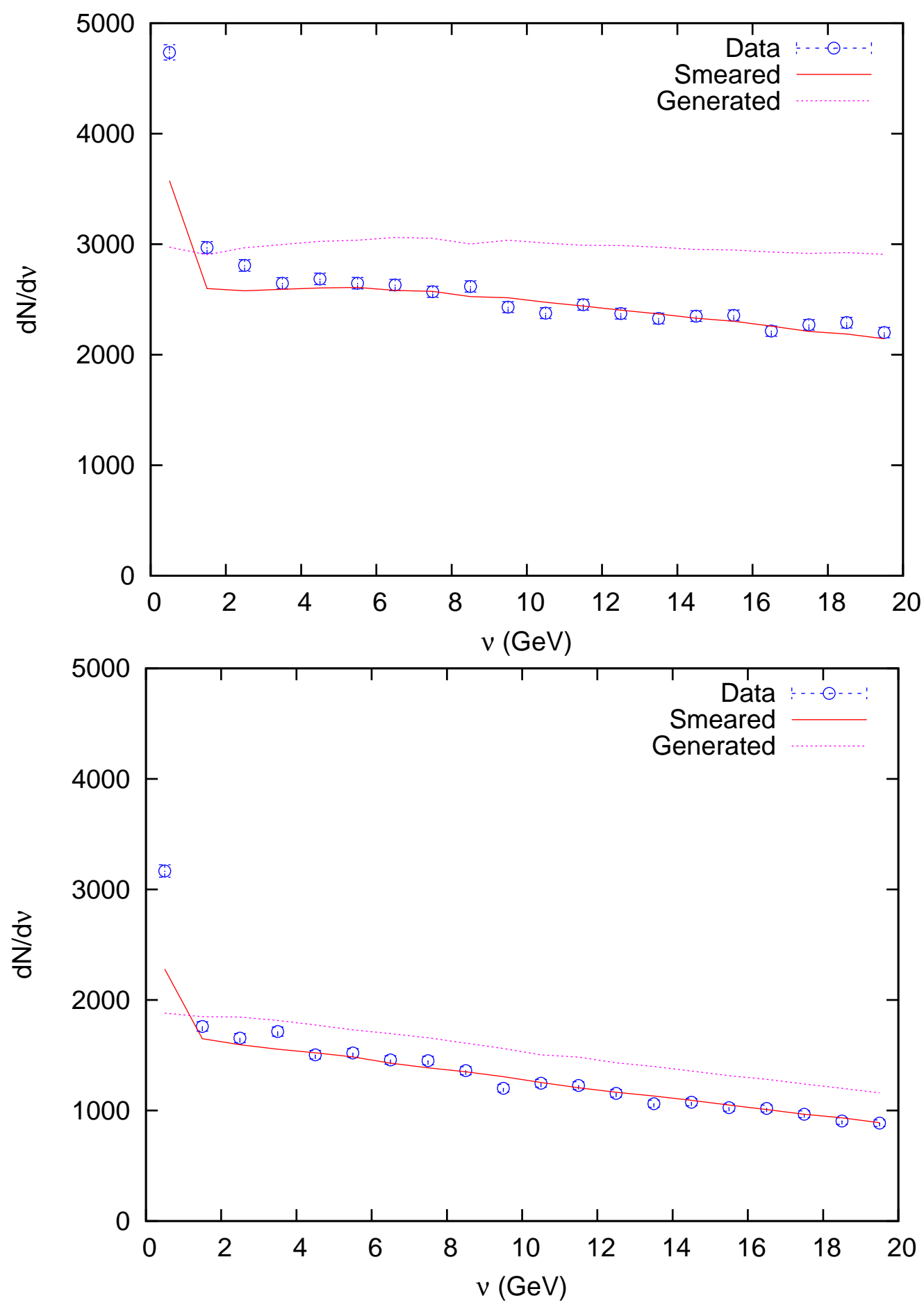

Figure 5.2: Comparison for $\frac{d N}{d \nu}$ from data (blue circles), Monte Carlo smeared (red line) and Monte Carlo generated (light blue line) for $E=75 \mathrm{GeV}$ for neutrinos (top) and anti-neutrinos (bottom). 


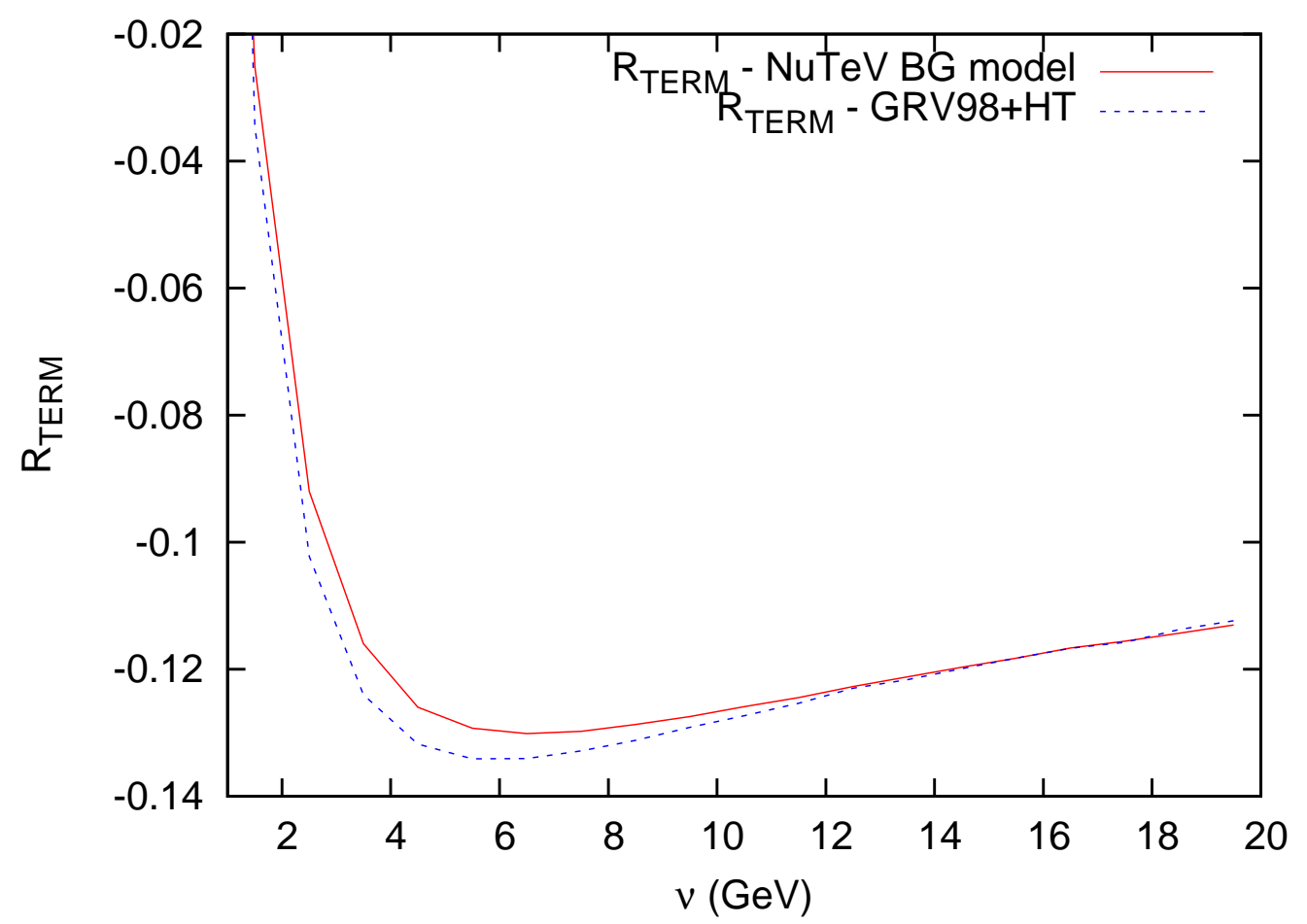

Figure 5.3: $R_{\text {TERM }}$ calculated using to different PDF set. NuTeV BG model (red solid line) and the model from reference [33] (blue dashed line). 

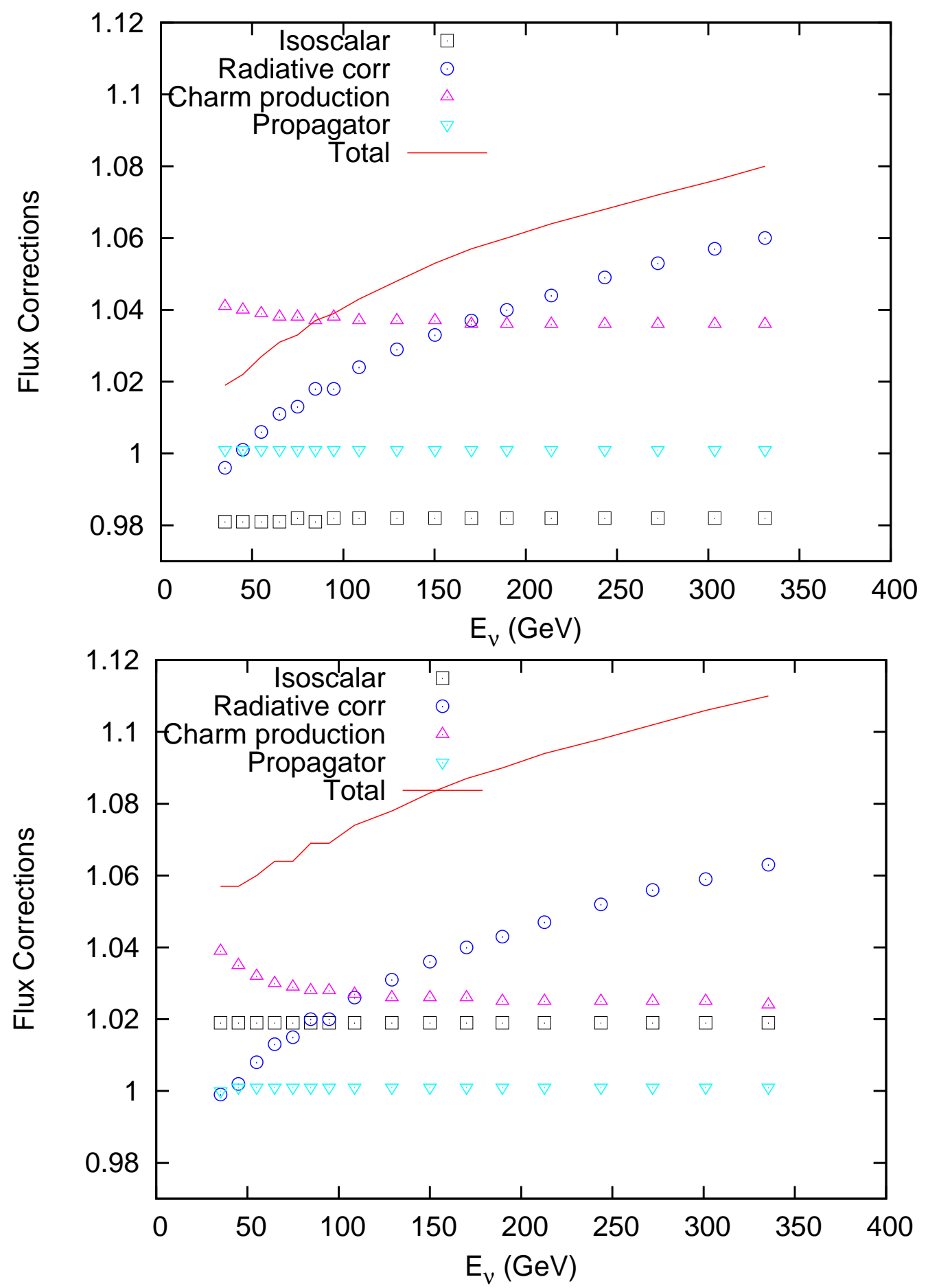

Figure 5.4: Physics corrections applied to the flux sample as function of $E_{\nu}$ for neutrinos (top) and anti-neutrinos (bottom). 
Figure 5.2 shows the the physics corrections in $E_{\nu}$ bins (integrated also over $\nu$ ) applied to the flux for neutrinos (top) and anti-neutrinos (bottom).

Figure 5.2 shows that as $\nu \rightarrow 0$ the number of events rises rapidly, because at low- $\nu$ there are events due to quasi-elastic, resonance production and the very low- $\nu$ part of the cross section. The cross section for these events is constant with energy, while the DIS cross section rises linearly with energy. Figure 5.5 shows the differential cross section $\frac{d \sigma}{d \nu}$ at low- $\nu$. A data sample which includes both DIS and low- $\nu$ events will have a different shape than that derived only for the DIS cross section. As a result low- $\nu$ events must be removed from the fitting sample for $B / A$. A low- $\nu$ cut $\nu>5 G e V$ is introduced in the fit to remove the non-DIS processes.

Since we fit the $\nu$ dependence we have to account for all $\nu$ dependent terms in the LHS of Equation 5.10. Previous analyses performed the fit under the assumption $A A$ and $B B$ are independent of $\nu$, which assumes that the scaling violations have small effect. As described in the previous section, scaling violations cause $A A$ and $B B$ to have an additional implicit $\nu$ dependence. In order to account for this effect the coefficients multiplying $A A$ and $B B$ are modified to include the $\nu$ dependence in Equation 5.10

$$
\begin{aligned}
\frac{d N^{D A T A_{C O R}}}{d \nu} & =A A \cdot\left(1-\frac{\nu}{E}+\frac{\nu^{2}}{2 E^{2}}\left(1+\frac{\int F_{2} R_{T E R M}}{\int F_{2}}\right)\right) \cdot \frac{\int F_{2}\left(x, Q^{2}=2 M x \nu\right) d x}{\int F_{2}\left(x, Q^{2}=2 M x \nu_{0}\right) d x} \\
& +B B \cdot\left(\frac{\nu}{E}-\frac{\nu^{2}}{2 E^{2}}\right) \cdot \frac{\int x F_{3}\left(x, Q^{2}=2 M x \nu\right) d x}{\int x F_{3}\left(x, Q^{2}=2 M x \nu_{0}\right) d x} .
\end{aligned}
$$

$\frac{d N}{d \nu}^{D A T A_{C O R R}}$ is the raw number of events corrected for acceptance and smearing, and for physics effects as discussed above (isoscalar target, radiative corrections, propagator and charm mass).

$B / A$ is determined from a fit to $\frac{d N}{d \nu}{ }^{D A T A_{C O R}}$ using Equation 5.12 in the interval $5 G e V<$ $\nu<20 \mathrm{GeV}$ for each energy bin. Figure 5.6 shows the $B / A$ fit for a typical energy bin $(E=75 \mathrm{GeV})$ for neutrinos and anti-neutrinos. The effect of the scaling violations on the slope is also shown. The fit value for $E=75 \mathrm{GeV}$ is $B / A=-0.30 \pm 0.10$ for neutrinos if the scaling violations correction is applied and $B / A=-0.47 \pm 0.11$ without the correction.

In order to minimize the statistical uncertainty a weighted average $\langle B / A\rangle$ is obtained for the energy range $40<E<260 \mathrm{GeV}$. The energy bin $30<E<40 \mathrm{GeV}$ is excluded from the 


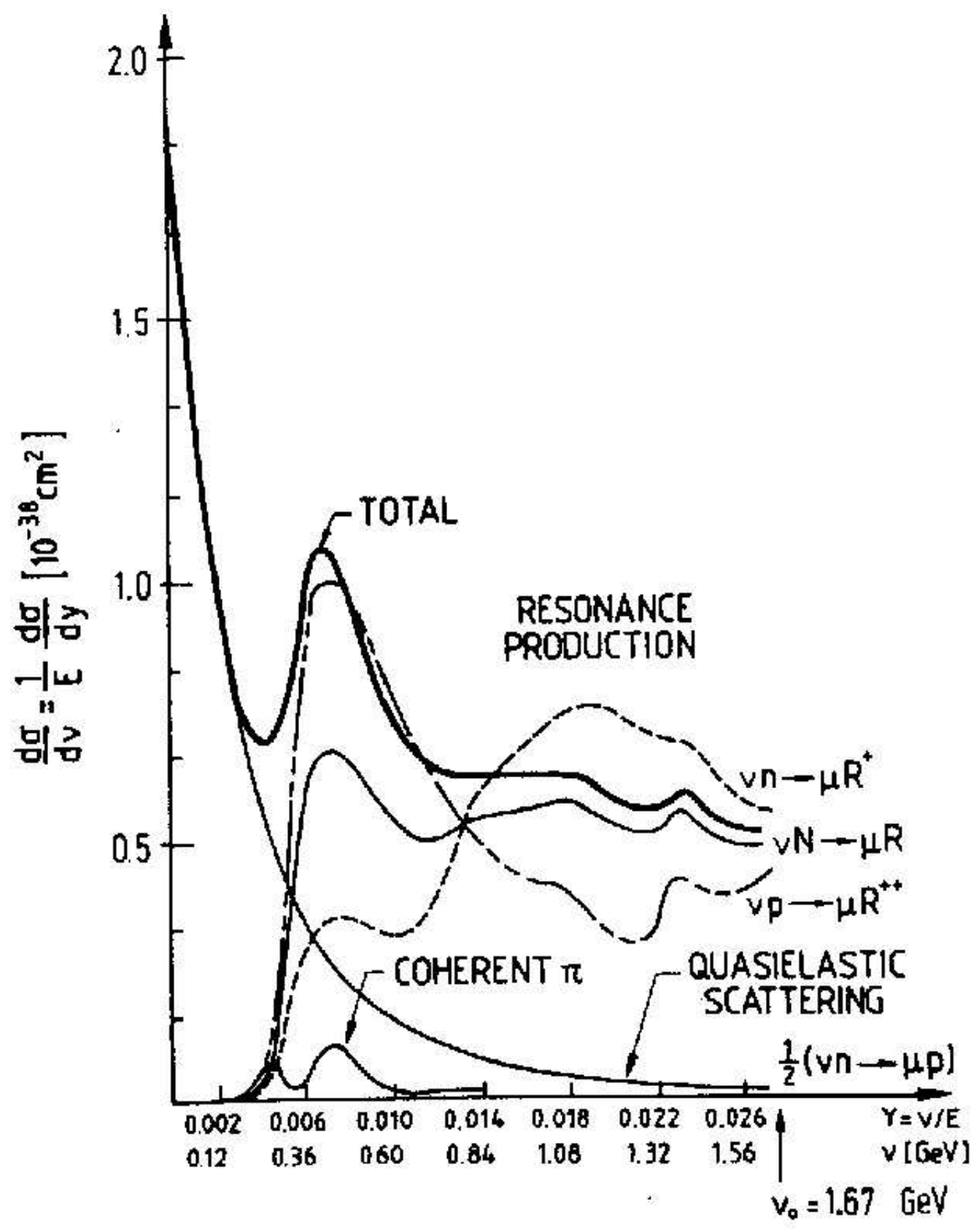

Figure 5.5: Differential cross section $d \sigma / d \nu$ as function of $\nu$ for $E=60 \mathrm{GeV}$. 

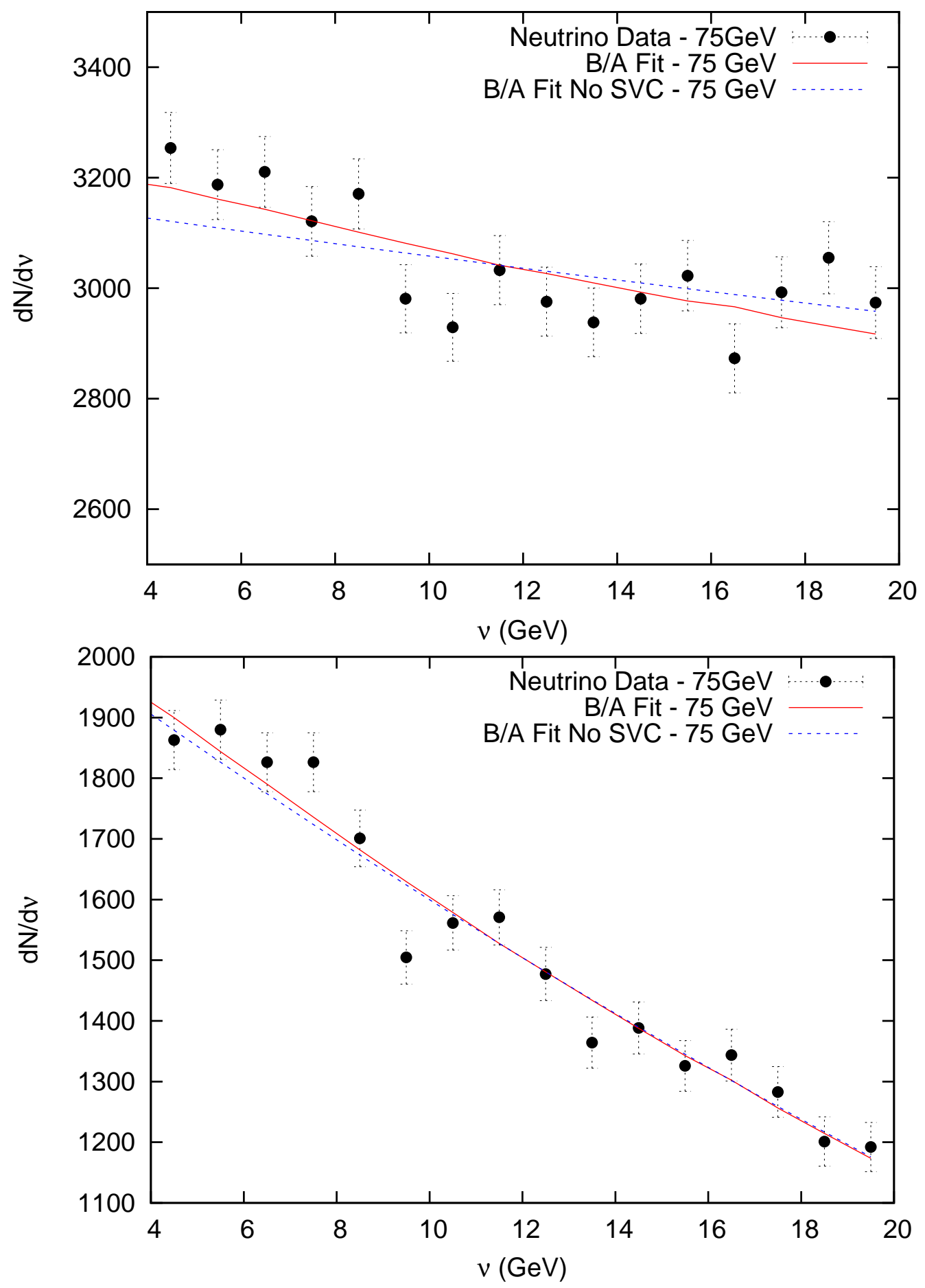

Figure 5.6: B/A fit to data for energy bin $(\mathrm{E}=75 \mathrm{GeV})$ for neutrinos (top) and anti-neutrinos (bottom). Since the $\nu$-dependence for DIS is different from that of quasi-elastics and resonances the fit is performed for data above $5 \mathrm{GeV}$. The blue dashed line shows the slope without scaling violations correction. 
fitting sample because $d N / d \nu$ from NUMONTE has a different slope than data due to poor bin population, which is a result of the low energy cuts. $\langle B / A\rangle^{\nu}=-0.34 \pm 0.04$ for neutrinos and $\langle B / A\rangle^{\bar{\nu}}=-1.68 \pm 0.03$ for anti-neutrinos. Figure 5.7 shows the fit values of $B / A$ for all energy bins for both neutrinos and anti-neutrinos. The line represents the weighted average. If the fit is performed without the scaling violations correction, $\langle B / A\rangle^{\nu}=-0.49 \pm 0.04$ for neutrinos and $\langle B / A\rangle^{\nu}=-1.71 \pm 0.03$ for anti-neutrinos.

Using $\langle B / A\rangle$ the $\nu / E$ correction is given by

$$
C O R_{\nu / E}=\frac{\int_{0}^{\nu_{0}} d \nu}{\int_{0}^{\nu_{0}} d \nu\left[1+\left\langle\frac{B}{A}\right\rangle\left(\frac{\nu}{E}-\frac{\nu^{2}}{2 E^{2}}\right)+\frac{\nu^{2}}{2 E^{2}} \frac{\int F_{2} R_{T E R M}}{\int F_{2}}\right]} .
$$

Figure 5.8 shows the size of $C O R_{\nu / E}$ as function of energy for both neutrinos and antineutrinos. $C O R_{\nu / E}$ becomes large at low energy for neutrinos, while for anti-neutrinos it is large for all energies.

Substituting $<B / A>$ and $\frac{\int F_{2} R_{T E R M}}{\int F_{2}}$ in Equation 5.8 gives the relative flux for both neutrinos and anti-neutrinos. Figure 5.9 shows the relative flux $E \cdot \Phi(E)$.

\subsubsection{Flux Systematic Uncertainties}

There are six systematic uncertainties which were evaluated for the relative flux measurement; the muon and hadron energy scales, the charm mass $m_{c}$, the $B / A$ fit value, and the muon and hadron energy smearing models. The effect of each of these uncertainties is evaluated as follows:

- Muon and Hadron energy scales - A shift in the muon energy scale will cause events to migrate to a neighboring kinematic bin and change the shape of the relative flux. In contrast a shift in the hadron energy scale will cause events near $E_{H A D}=20 \mathrm{GeV}$ to migrate into or out of the flux sample. This effect will be similar for all energy bins, therefore, the effect on the relative flux will be small. The muon and hadron energy scale uncertainties are taken to be $\pm 0.7 \%$ and $\pm 0.43 \%$ respectively.

- $\langle B / A\rangle$ fit values - A change in the $\langle B / A\rangle$ value, which multiplies $\nu / E$ to correct the flux, will change the energy dependence and therefore change the relative flux shape. There are two contributions to the $\langle B / A\rangle$ uncertainty, the statistical error from the fit $( \pm 0.04$ for 


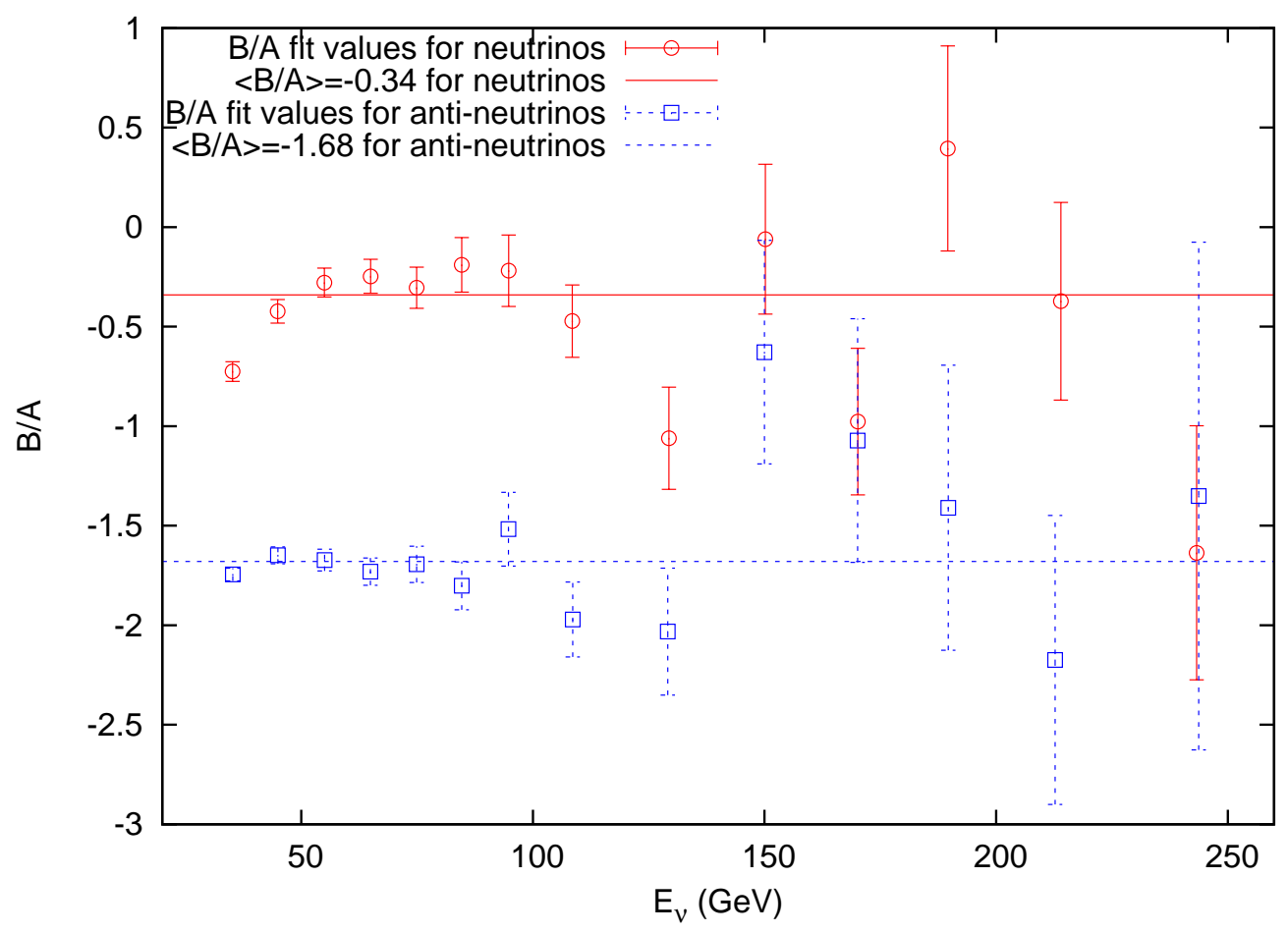

Figure 5.7: $\mathrm{B} / \mathrm{A}$ fit value as function of energy. $B / A$ is flat with energy for both neutrinos and anti-neutrinos. $\langle B / A\rangle^{\nu}=-0.34 \pm 0.04$ for neutrinos. $\langle B / A\rangle^{\bar{\nu}}=-1.68 \pm 0.03$ for anti-neutrinos. 


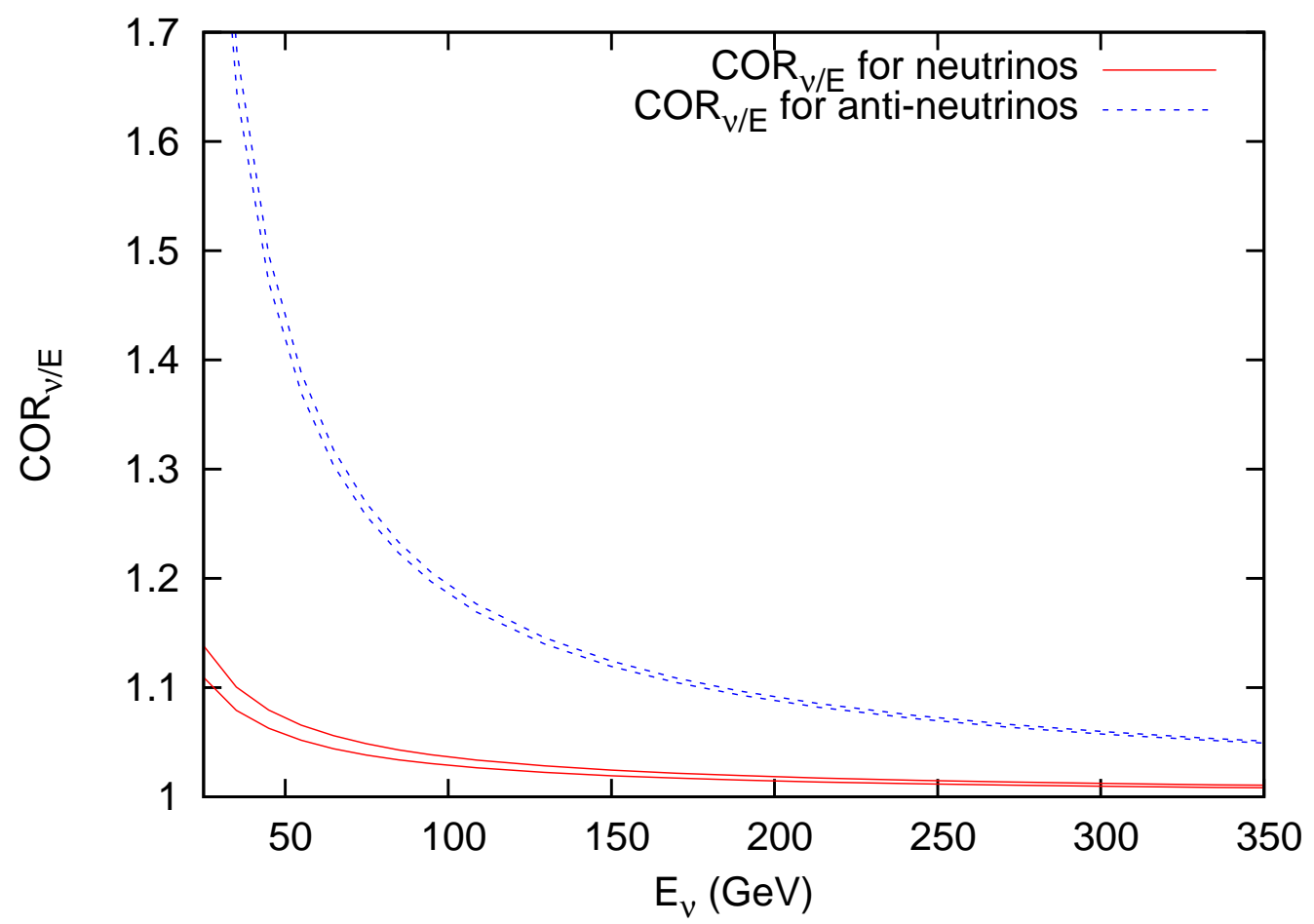

Figure 5.8: Correction applied to neutrino (red solid line) and anti-neutrino (blue dashed line) fluxes to account for the energy dependence. 


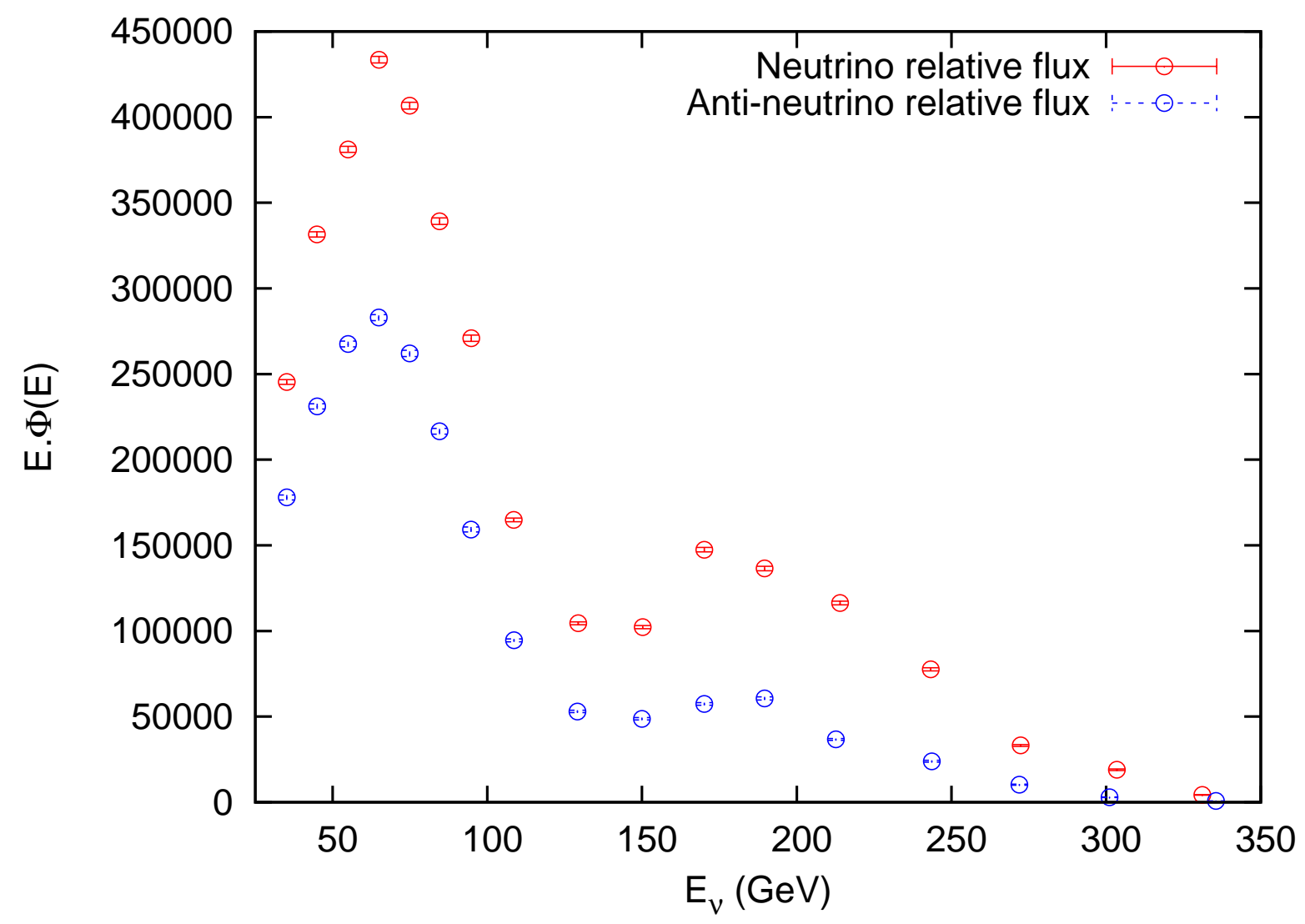

Figure 5.9: Relative flux for neutrinos (red circles) and anti-neutrinos (blue circles). Systematic errors are included in quadrature. 
neutrinos, \pm 0.03 for anti-neutrinos), and a model uncertainty in computing the scaling violations correction. Using an alternative model [33] to compute the scaling violations corrections and the acceptance correction we obtained a value for $\langle B / A\rangle=-0.39$ for neutrinos and $\langle B / A\rangle=-1.72$ for anti-neutrinos. The difference between the two models is taken as the systematic uncertainty in $\langle B / A\rangle$.

- Charm mass - The slow rescaling model described in Section 4.1.3.1 is used to model the charm production cross section. Because charm production introduces an energy dependence into the cross section we correct $d N / d \nu$ to remove this effect (Equation 5.11). Thus the extracted flux depends on the charm mass value. Different charm mass value will change the relative flux shape. The charm mass uncertainty is taken to be \pm 0.18 (see Section 4.1.3.1).

- Muon and Hadron energy smearing models - Smearing models will affect the acceptance corrections from our Monte Carlo. The error on the parameters of the hadron energy smearing model are used as the value for the systematic uncertainty on hadron energy smearing (see Section 3.1). In order to evaluate the systematic uncertainty due to the muon smearing model we use an alternative model given by Equation 3.20 which has the same functional form but the width of the leading Gaussian is parameterized as a linear function with energy (see Section 3.21).

The uncertainty in the flux due to each of the systematic uncertainties discussed above is evaluated using the following procedure:

1. If a systematic uncertainty has a symmetric value, $\left(E_{\mu}\right.$ and $E_{H A D}, m_{c},\langle B / A\rangle$ statistical uncertainty and $E_{H A D}$ smearing), we shift the central value up and down by the uncertainty size. New fluxes are extracted with the shifted values and the symmetrized difference is taken to be the evaluated systematic uncertainty on the flux measurement.

2. If a systematic is evaluated using an alternative model, $\left(E_{\mu}\right.$ smearing, $\langle B / A\rangle$ systematic uncertainty), a new flux is extracted with the alternative model. The difference between the default and the alternative models is taken to be the systematic uncertainty. 


\subsection{TOTAL NEUTRINO CROSS SECTION AND FLUX NORMALIZATION}

Total neutrino and anti-neutrino cross sections are extracted by defining a sample with a good muon track in the detector. There are 1147281 events for neutrinos and 386683 for antineutrinos in the cross section sample (see Section 3.4). The raw number of events, binned in energy bins, are shown in Table 5.2. An correction, $A C_{i}^{X S E C}$, is generated for each energy bin using our Monte Carlo to correct the raw number of events for detector acceptance and smearing

$$
N_{i}^{D A T A_{C O R}}=N_{i}^{D A T A_{R A W}} \cdot A C_{i}^{X S E C}=N_{i}^{D A T A_{R A W}} \cdot \frac{N_{i}^{G E N_{X S E C}}}{N_{i}^{S M E_{X S E C}}}
$$

where $N_{i}^{G E N_{X S E C}}$ and $N_{i}^{S M E_{X S E C}}$ are the generated and the smeared number of events for this energy bin. Systematic uncertainties for the unnormalized cross section are obtained with the same procedure used for the flux (Section 5.2.1).

Since we know the relative flux we can extract the total cross section

$$
\frac{\sigma}{E_{i}}=\frac{N_{i}^{D A T A_{C O R}}}{E_{i} \cdot \Phi\left(E_{i}\right)}
$$

Absolute flux is obtained by normalizing the relative neutrino cross section to the world average neutrino cross section for an isoscalar target $\frac{\sigma_{W O R L D_{I S O}}^{\nu}}{E}=0.677 \pm 0.014 \times 10^{-38} \mathrm{~cm}^{2} / \mathrm{GeV}$. The world average cross section uncertainty of $2.1 \%$, will be treated as a systematic uncertainty on the flux normalization. In order to normalize the relative neutrino cross section an isoscalar correction is applied $C O R_{i}^{I S O} \cdot \frac{\sigma}{E}{ }_{i}$, where

$$
C O R_{i}^{I S O}=\frac{\int_{0}^{1} \int_{0}^{1} d x d y \frac{d^{2} \sigma(i s o)}{d x d y}\left(x, y, E_{i}\right)}{\int_{0}^{1} \int_{0}^{1} d x d y \frac{d^{2} \sigma(F e)}{d x d y}\left(x, y, E_{i}\right)}
$$

Figure 5.10 shows the isoscalar correction applied to the total neutrino and anti-neutrino cross sections. Since the world average cross section is obtained from data in the energy range $30-200 \mathrm{GeV}$, the weighted average of the isoscalar neutrino total cross section $\left\langle\frac{\sigma^{I S O}}{E}\right\rangle_{30-200 \mathrm{GeV}}$ is normalized to $\frac{\sigma_{W O R L D_{I S O}}^{\nu}}{E}=0.677 \times 10^{-38} \mathrm{~cm}^{2} / \mathrm{GeV}$. The same normalization is used for the anti-neutrino flux. Figure 5.11 shows the $\mathrm{NuTeV}$ isoscalar neutrino and anti-neutrino cross sections. The error bars represent the statistical uncertainty and the 


\begin{tabular}{|c|c|c|c|}
\hline Energy bin & Bin limits & Num. of events $\nu$-mode & Num. of events $\bar{\nu}$-mode \\
\hline 1 & $30-40 \mathrm{GeV}$ & 42573 & 21916 \\
\hline 2 & $40-50 \mathrm{GeV}$ & 64972 & 31040 \\
\hline 3 & $50-60 \mathrm{GeV}$ & 83586 & 38036 \\
\hline 4 & $60-70 \mathrm{GeV}$ & 97421 & 42049 \\
\hline 5 & $70-80 \mathrm{GeV}$ & 97898 & 39885 \\
\hline 6 & $80-90 \mathrm{GeV}$ & 86619 & 33606 \\
\hline 7 & $90-100 \mathrm{GeV}$ & 69072 & 25300 \\
\hline 8 & $100-120 \mathrm{GeV}$ & 88805 & 31292 \\
\hline 9 & $120-140 \mathrm{GeV}$ & 56394 & 18028 \\
\hline 10 & $140-160 \mathrm{GeV}$ & 58157 & 16388 \\
\hline 11 & $160-180 \mathrm{GeV}$ & 70806 & 18133 \\
\hline 12 & $180-200 \mathrm{GeV}$ & 76444 & 17692 \\
\hline 13 & $200-230 \mathrm{GeV}$ & 99882 & 20204 \\
\hline 14 & $230-260 \mathrm{GeV}$ & 66740 & 11716 \\
\hline 15 & $260-290 \mathrm{GeV}$ & 36883 & 5715 \\
\hline 16 & $290-320 \mathrm{GeV}$ & 17407 & 2337 \\
\hline 17 & $320-360 \mathrm{GeV}$ & 8692 & 1100 \\
\hline
\end{tabular}

Table 5.2: Raw number of events in neutrino energy bins passing total cross section cuts for neutrinos and anti-neutrinos. 


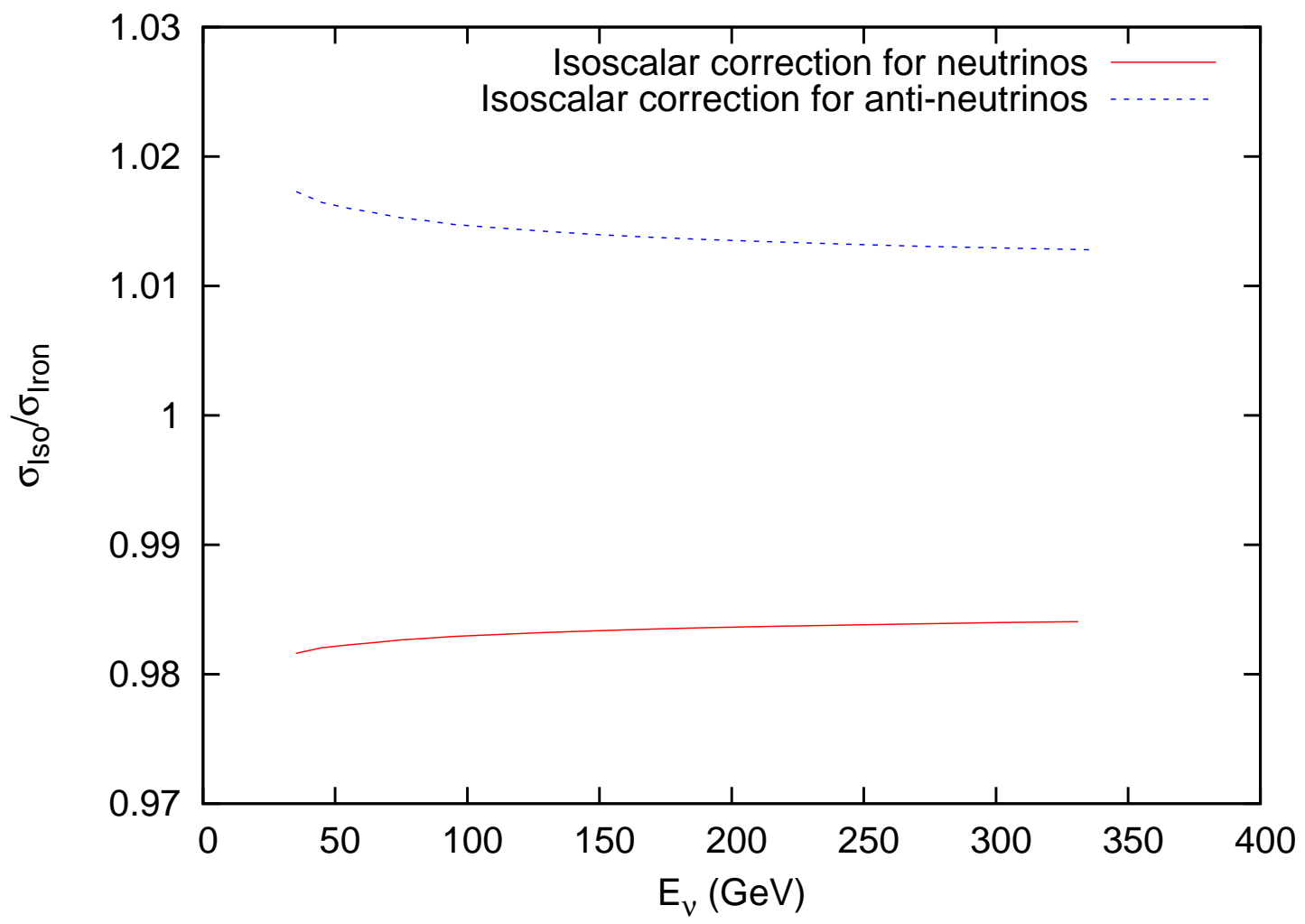

Figure 5.10: Isoscalar correction applied to the total neutrino (solid red curve) and antineutrino (dashed blue curve) cross sections as functions of $E_{\nu}$. 
yellow band represents the total systematic uncertainty (excluding the flux normalization uncertainty). Table 5.4 shows the value of the normalized cross section with statistical and systematic error for all energy bins.

\subsection{ANTI-NEUTRINO TO NEUTRINO CROSS SECTION RATIO}

Since we use the same normalization for the relative neutrino and anti-neutrino fluxes, it will

cancel when we form the ratio $r^{\nu}=\frac{\sigma^{\bar{\nu}}}{\sigma^{\nu}}$. Therefore $\mathrm{NuTeV}$ can measure the absolute level of $r^{\nu}$, which is computed for each energy bin, given in Table 5.3.

The weighted average computed using the statistical uncertainty for the energy range $30-360 \mathrm{GeV}$ is

$$
\left\langle r^{\nu}\right\rangle_{30-360 \mathrm{GeV}}=0.505 \pm 0.0018(\text { stat }) \pm 0.0029(\text { syst })
$$

The systematic uncertainty is computed by propagating the uncertainty in the flux and the cross section samples. In order to compare with previous measurements we computed the average for the energy range $30-200 \mathrm{GeV}$

$$
\left\langle r^{\nu}\right\rangle_{30-200 G e V}=0.505 \pm 0.0018(\text { stat }) \pm 0.0029(\text { syst })
$$

The world average before $\mathrm{NuTeV}$ is $\left\langle r^{\nu}\right\rangle_{30-200 \mathrm{GeV}}^{\text {World }}=0.499 \pm 0.007$. Figure 5.4 shows the measured $r^{\nu}$ as function of energy $E_{\nu}$ and $\left\langle r^{\nu}\right\rangle$ for the energy range 30-200 GeV compared with the world average. NuTeV agrees with the world average. NuTeV's result gives the most precise measurement of the ratio of anti-neutrino to neutrino total cross sections. 


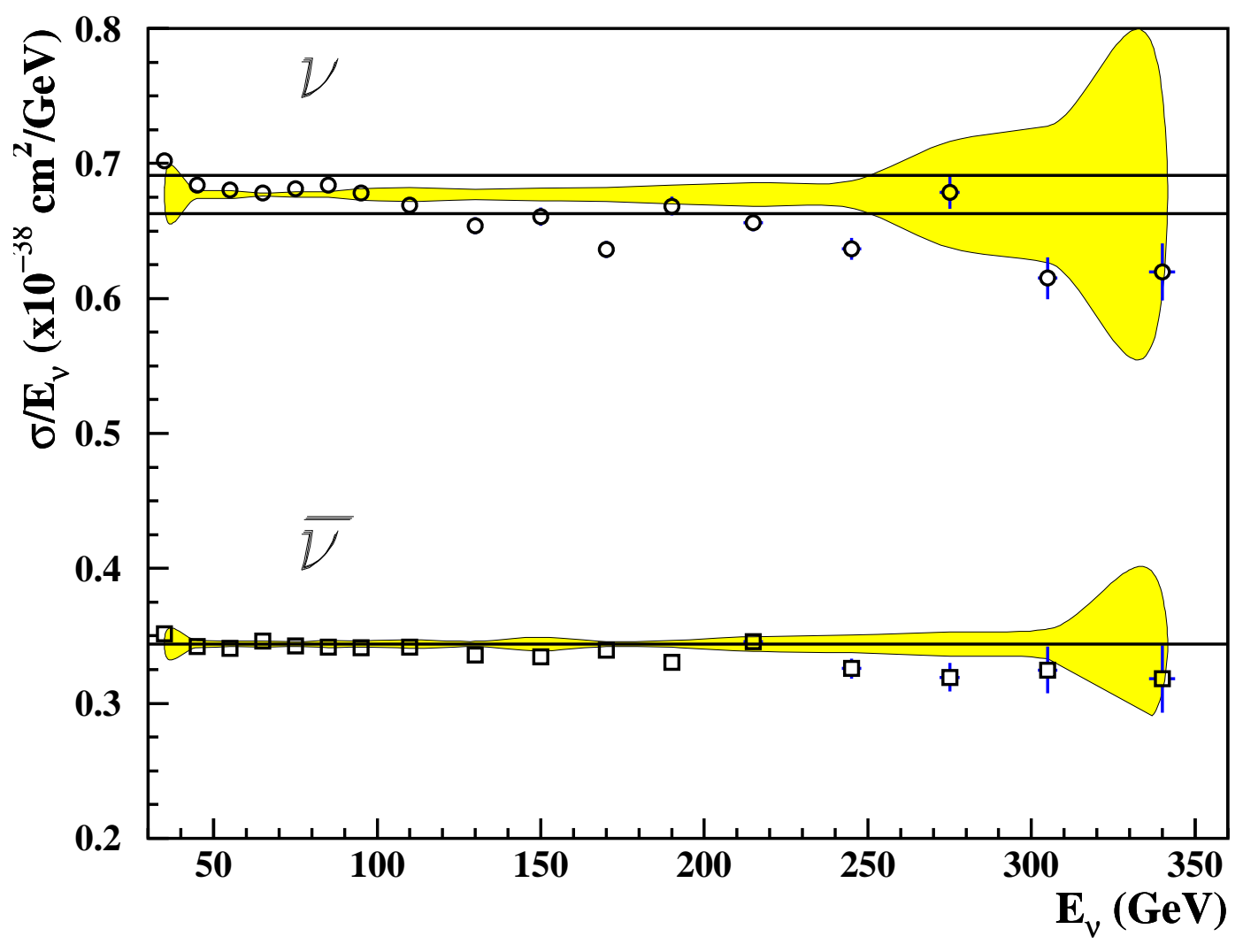

Figure 5.11: Total isoscalar neutrino (circles) and anti-neutrino (squares) cross section for scattering off an iron target. Data is shown with its statistical uncertainty. The yellow band shows the systematic uncertainty (excluding the flux normalization uncertainty. The lines represent $2.1 \%$ uncertainty of the world average cross section. 


\begin{tabular}{|c|c|c|}
\hline Bin limits in GeV & $\frac{\sigma_{I S O}^{\nu} \pm \text { Stat } \pm \text { Syst }}{E}$ & $\frac{\sigma_{I S O}^{\bar{\nu}}}{E} \pm$ Stat \pm Syst \\
\hline $30-40$ & $0.7021 \pm 0.0054 \pm 0.0065$ & $0.3514 \pm 0.0038 \pm 0.0069$ \\
\hline $40-50$ & $0.6840 \pm 0.0043 \pm 0.0035$ & $0.34220 \pm .0030 \pm 0.0041$ \\
\hline $50-60$ & $0.6802 \pm 0.0040 \pm 0.0030$ & $0.3409 \pm 0.0028 \pm 0.0028$ \\
\hline $60-70$ & $0.6783 \pm 0.0039 \pm 0.0012$ & $0.3464 \pm 0.0028 \pm 0.0024$ \\
\hline $70-80$ & $0.6811 \pm 0.0042 \pm 0.0023$ & $0.3427 \pm 0.0029 \pm 0.0017$ \\
\hline $80-90$ & $0.6841 \pm 0.0047 \pm 0.0029$ & $0.3417 \pm 0.0033 \pm 0.0031$ \\
\hline $90-100$ & $0.6781 \pm 0.0055 \pm 0.0042$ & $0.3412 \pm 0.0039 \pm 0.0020$ \\
\hline $100-120$ & $0.6693 \pm 0.0050 \pm 0.0050$ & $0.3416 \pm 0.0037 \pm 0.0034$ \\
\hline $120-140$ & $0.6538 \pm 0.0065 \pm 0.0041$ & $0.3356 \pm 0.0049 \pm 0.0023$ \\
\hline $140-160$ & $0.6605 \pm 0.0069 \pm 0.0044$ & $0.3347 \pm 0.0054 \pm 0.0053$ \\
\hline $160-180$ & $0.6362 \pm 0.0064 \pm 0.0059$ & $0.3396 \pm 0.0055 \pm 0.0025$ \\
\hline $180-200$ & $0.6683 \pm 0.0070 \pm 0.0079$ & $0.3303 \pm 0.0057 \pm 0.0028$ \\
\hline $200-230$ & $0.6562 \pm 0.0064 \pm 0.0103$ & $0.3457 \pm 0.0059 \pm 0.0057$ \\
\hline $230-260$ & $0.6366 \pm 0.0081 \pm 0.0105$ & $0.3257 \pm 0.0076 \pm 0.0072$ \\
\hline $260-290$ & $0.6786 \pm 0.0121 \pm 0.0303$ & $0.3193 \pm 0.0107 \pm 0.0072$ \\
\hline $290-320$ & $0.6151 \pm 0.0155 \pm 0.0508$ & $0.3247 \pm 0.0173 \pm 0.0099$ \\
\hline $320-360$ & $0.6197 \pm 0.0213 \pm 0.0766$ & $0.3181 \pm 0.0250 \pm 0.0396$ \\
\hline
\end{tabular}

Table 5.3: Neutrino and anti-neutrino total cross sections for all energy bins in units $10^{-38} \mathrm{~cm}^{2} / \mathrm{GeV}$. Both the statistical and the systematic errors are shown for each bin excluding the normalization uncertainty. 


\begin{tabular}{|c|c|c|c|}
\hline Bin limits in GeV & $r^{\nu}=\frac{\sigma^{\bar{\nu}}}{\sigma^{\nu}}$ & Stat. error & Syst. error \\
\hline $30-40$ & 0.50046 & 0.00667 & 0.00691 \\
\hline $40-50$ & 0.50023 & 0.00544 & 0.00491 \\
\hline $50-60$ & 0.50121 & 0.00505 & 0.00356 \\
\hline $60-70$ & 0.51066 & 0.00506 & 0.00312 \\
\hline $70-80$ & 0.50316 & 0.00528 & 0.00215 \\
\hline $80-90$ & 0.49955 & 0.00591 & 0.00395 \\
\hline $90-100$ & 0.50313 & 0.00707 & 0.00461 \\
\hline $100-120$ & 0.51033 & 0.00667 & 0.00517 \\
\hline $120-140$ & 0.51331 & 0.00904 & 0.00293 \\
\hline $140-160$ & 0.50667 & 0.00974 & 0.01011 \\
\hline $160-180$ & 0.53376 & 0.01020 & 0.00373 \\
\hline $180-200$ & 0.49426 & 0.01000 & 0.00412 \\
\hline $200-230$ & 0.52675 & 0.01040 & 0.00527 \\
\hline $230-260$ & 0.51162 & 0.01356 & 0.00648 \\
\hline $260-290$ & 0.47056 & 0.01787 & 0.01416 \\
\hline $290-320$ & 0.52787 & 0.03105 & 0.04575 \\
\hline $320-360$ & 0.51341 & 0.04401 & 0.11522 \\
\hline
\end{tabular}

Table 5.4: Anti-neutrino to neutrino total cross section ratio for all energy bins. Both the statistical and the systematic errors are shown for each bin. 


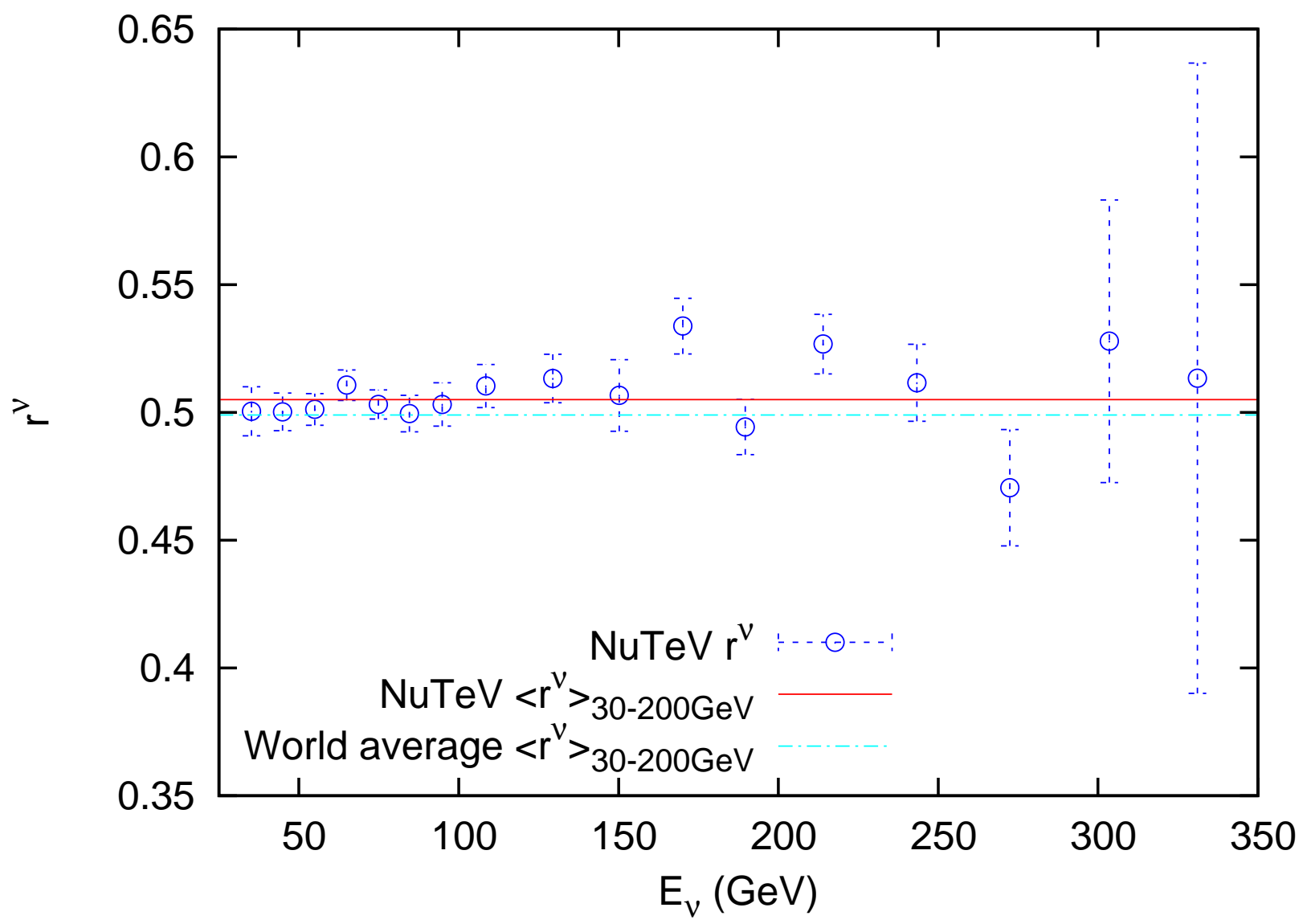

Figure 5.12: The ratio of anti-neutrino to neutrino total cross sections (blue circles). The world average value (light blue line) $0.499 \pm 0.007$ is compared with the $\mathrm{NuTeV}$ average (red line) $0.505 \pm 0.0015$ (stat) \pm 0.0029 (syst) for the same energy range $30-200 \mathrm{GeV}$. Systematic errors are included. 


\subsection{DIFFERENTIAL CROSS SECTION}

The number of events, $N_{\left(\begin{array}{l}x_{0} \leq x \leq x_{1} \\ y_{0} \leq y \leq y_{1}\end{array}\right)}$ in the kinematic range $x_{0} \leq x \leq x_{1}, y_{0} \leq y \leq y_{1}$ depends on the average differential cross section, $\left\langle\frac{d^{2} \sigma}{d x d y}\right\rangle_{\substack{x_{0} \leq x \leq x_{1} \\ y_{0} \leq y \leq y_{1}}}=\frac{1}{\Delta x \Delta y} \int_{x_{0}}^{x_{1}} \int_{y_{0}}^{y_{1}} d x d y \frac{d^{2} \sigma}{d x d y}$, and the flux, $\Phi(E)$,

$$
\begin{aligned}
N_{\left(\begin{array}{c}
x_{0} \leq x \leq x_{1} \\
y_{0} \leq y \leq y_{1}
\end{array}\right)} & \left.=\rho L N_{A} \Delta x \Delta y \int d E \Phi(E)\left\langle\frac{d^{2} \sigma}{d x d y}\right\rangle_{\substack{x_{0} \leq x \leq x_{1} \\
y_{0} \leq y \leq y_{1}}}\right) \\
& \propto \int d E \Phi(E) \int_{x_{0}}^{x_{1}} \int_{y_{0}}^{y_{1}} d x d y \frac{d^{2} \sigma}{d x d y}(x, y, E),
\end{aligned}
$$

where $\rho$ is the target density, $L$ is the target length, $N_{A}$ is Avogadro's number and $\Delta x=$ $x_{1}-x_{0}, \Delta y=y_{1}-y_{0}$. In this chapter, a measurement of the differential cross section for inclusive charge current neutrino iron scattering is presented.

\subsection{DIFFERENTIAL CROSS SECTION EXTRACTION}

The differential cross section is extracted from the structure function sample which contains charged-current events with a high energy hadron shower and single muon (see Section 3.4). There are 866000 neutrino and 240000 anti-neutrino events in this sample. The events are binned in twelve $x$ bins, eleven $y$ bins and seventeen $E_{\nu}$ bins. The energy bins have the same size and limits as the flux energy bins. The size of the bins in $\mathrm{x}$ and $\mathrm{y}$ are chosen based on event population and detector resolution. Table 6.1 shows the bin limits for $\mathrm{x}, \mathrm{y}$ and E. The lower limits of the lowest $\mathrm{x}$ and $\mathrm{y}$ bins take into account the kinematic limits due to the non-zero mass of the muon. The number of raw events, $D A T A_{i j k}^{D X E C}$, in each $\mathrm{x}, \mathrm{y}, \mathrm{E}$ bin is corrected for detector acceptance and smearing effects using NUMONTE. A Monte Carlo 


\begin{tabular}{|c|c|c|c|}
\hline Bin number & $E_{\nu}$ bin limits & $x$ bin limits & $y$ bin limits \\
\hline 1 & $30-40 \mathrm{GeV}$ & $0.0001-0.03$ & $0.001-0.05$ \\
\hline 2 & $40-50 \mathrm{GeV}$ & $0.03-0.06$ & $0.05-0.10$ \\
\hline 3 & $50-60 \mathrm{GeV}$ & $0.06-0.10$ & $0.10-0.20$ \\
\hline 4 & $60-70 \mathrm{GeV}$ & $0.10-0.15$ & $0.20-0.30$ \\
\hline 5 & $70-80 \mathrm{GeV}$ & $0.15-0.20$ & $0.30-0.40$ \\
\hline 6 & $80-90 \mathrm{GeV}$ & $0.20-0.25$ & $0.40-0.50$ \\
\hline 7 & $90-100 \mathrm{GeV}$ & $0.25-0.30$ & $0.50-0.60$ \\
\hline 8 & $100-120 \mathrm{GeV}$ & $0.30-0.40$ & $0.60-0.70$ \\
\hline 9 & $120-140 \mathrm{GeV}$ & $0.40-0.50$ & $0.70-0.80$ \\
\hline 10 & $140-160 \mathrm{GeV}$ & $0.50-0.60$ & $0.80-0.90$ \\
\hline 11 & $160-180 \mathrm{GeV}$ & $0.60-0.70$ & $0.90-0.97$ \\
\hline 12 & $180-200 \mathrm{GeV}$ & $0.70-0.80$ & \\
\hline 13 & $200-230 \mathrm{GeV}$ & & \\
\hline 14 & $230-260 \mathrm{GeV}$ & & \\
\hline 15 & $260-290 \mathrm{GeV}$ & & \\
\hline 16 & $290-320 \mathrm{GeV}$ & & \\
\hline 17 & $320-360 \mathrm{GeV}$ & & \\
\hline
\end{tabular}

Table 6.1: Bin limits for $E_{\nu}, x$ and $y$. 
sample is generated with statistics about 20 times that of data. The acceptance correction for each bin $(\mathrm{i}, \mathrm{j}, \mathrm{k})$ is given by

$$
A C_{i j k}^{D X E C}=\frac{G E N_{i j k}^{D X E C}}{S M E_{i j k}^{D X E C}}
$$

where $G E N_{i j k}^{D X E C}$ is the true number of events and $S M E_{i j k}^{D X E C}$ is the SMEARED number of events. The average differential cross section for bin $(i, j, k)$ is

$$
\left\langle\frac{d^{2} \sigma}{E d x d y}\right\rangle_{i j k}=\frac{1}{E_{k} \cdot \Phi\left(E_{k}\right) \Delta x_{i} \Delta y_{j}} \cdot D A T A_{i j k}^{D X E C} A C_{i j k}^{D X E C},
$$

where $\Delta x_{i}=x_{i+1}-x_{i}$ and $\Delta y_{j}=y_{j+1}-y_{j}$. Figures 6.1 and 6.2 show the acceptance, $1 / A C_{i j k}^{D X E C}$, for two energy bins $E=65 \mathrm{GeV}$ and $E=110 \mathrm{GeV}$ respectively in $\mathrm{x}$ bins as a function of $y$,

A muon is required to penetrate at least in two gaps in the toroid. This requirement will affect the acceptance of low energy muons, which scatter at large angles. Therefore we have poor acceptance at high y. The acceptance at low y is affected by the cut $E_{H A D}>10 \mathrm{GeV}$. Acceptance corrections for neutrinos and anti-neutrinos are similar, because in both modes the toroid polarity is chosen so that the primary muon is focused.

The extracted cross section table represents the average differential cross section for each kinematic bin and therefore is not associated with a specific kinematic point, $(\mathrm{x}, \mathrm{y}, \mathrm{E})$, in the bin. In order to associate the cross section with a particular point we apply a bin centering correction, $B C_{i j k}$, which is calculated using our cross section model. This correction is the ratio of the cross section value at the center of a bin, $\left(x_{i}^{c}, y_{j}^{c}, E_{k}^{c}\right)$, to the average cross section over the bin

$$
B C_{i j k}=\frac{\frac{d^{2} \sigma}{E d x d y}\left(x_{i}^{c}, y_{j}^{c}, E_{k}^{c}\right)}{\frac{1}{\Delta x_{i} \Delta y_{j}} \int_{x_{i}}^{x_{i+1}} \int_{y_{j}}^{y_{j+1}} d x d y \frac{d^{2} \sigma}{E d x d y}(x, y, E)},
$$

where $x_{i}^{c}=\left(x_{i+1}+x_{i}\right) / 2, y_{j}^{c}=\left(y_{j+1}+y_{j}\right) / 2$ and $E_{k}^{c}=\left(E_{k+1}+E_{k}\right) / 2$. Figures 6.3 and 6.4 show the bin centering correction in $\mathrm{x}$ bins as function of $\mathrm{y}$ for $\mathrm{E}=55 \mathrm{GeV}$ and $\mathrm{E}=110 \mathrm{GeV}$ respectively. The bin-centered cross section is obtained from

$$
\frac{d^{2} \sigma}{E d x d y}\left(x_{i}^{c}, y_{j}^{c}, E_{k}^{c}\right)=\left\langle\frac{d^{2} \sigma}{E d x d y}\right\rangle_{i j k} \cdot B C_{i j k}
$$




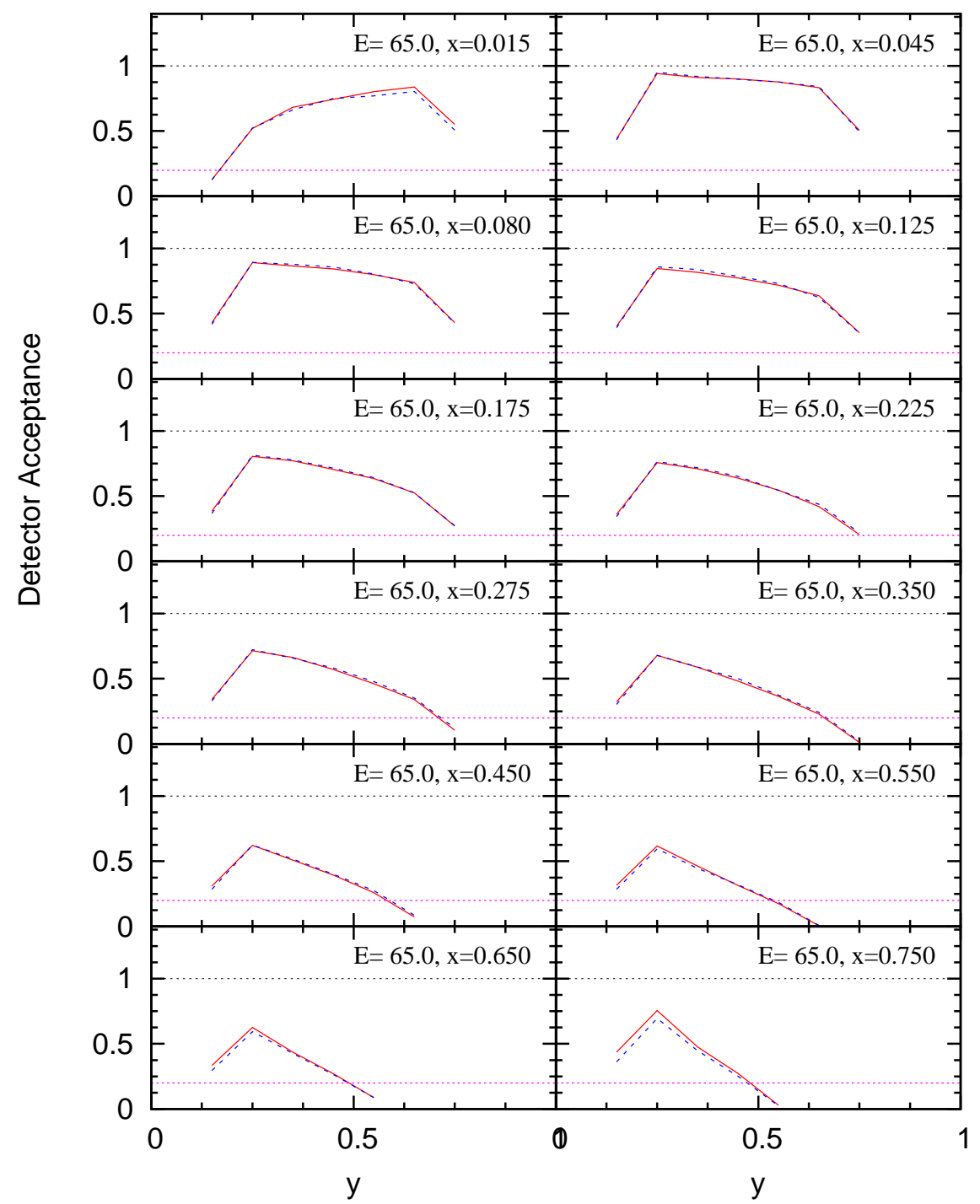

Acceptance for Neutrinos

Acceptance for Anti-Neutrinos

Min. Acceptance

Figure 6.1: Acceptance (red solid curve) for $\mathrm{E}=65 \mathrm{GeV}$ in $\mathrm{x}$ bins as a function of $\mathrm{y}$ for neutrinos (solid red curve) and anti-neutrinos (dashed blue curve). The minimum acceptance of $20 \%$ is shown with blue dashed line. 


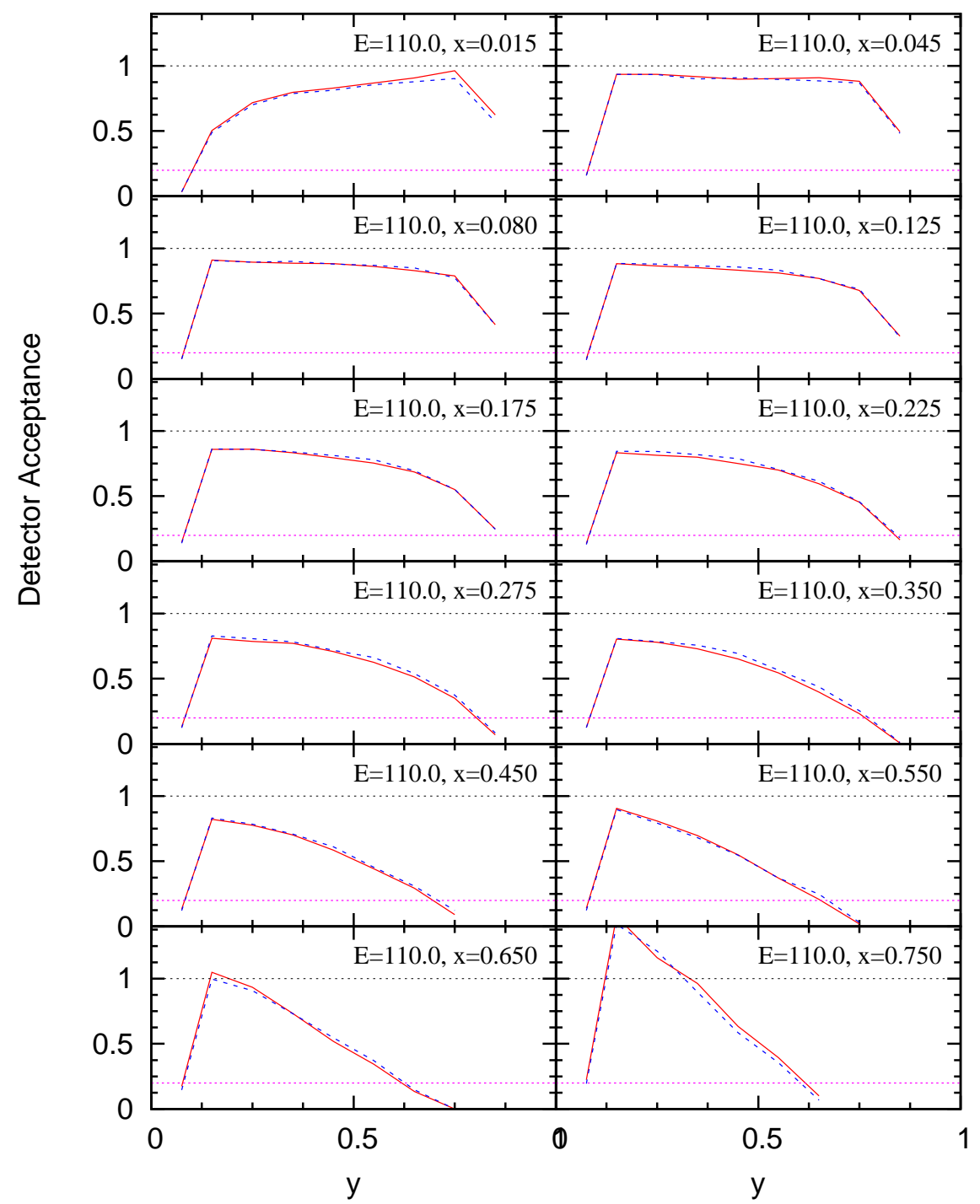

Acceptance for Neutrinos

Acceptance for Anti-Neutrinos

Min. Acceptance

Figure 6.2: Acceptance (red solid curve) for $\mathrm{E}=110 \mathrm{GeV}$ in $\mathrm{x}$ bins as a function of $\mathrm{y}$ for neutrinos (solid red curve) and anti-neutrinos (dashed blue curve). The minimum acceptance of $20 \%$ is shown with blue dashed line. 


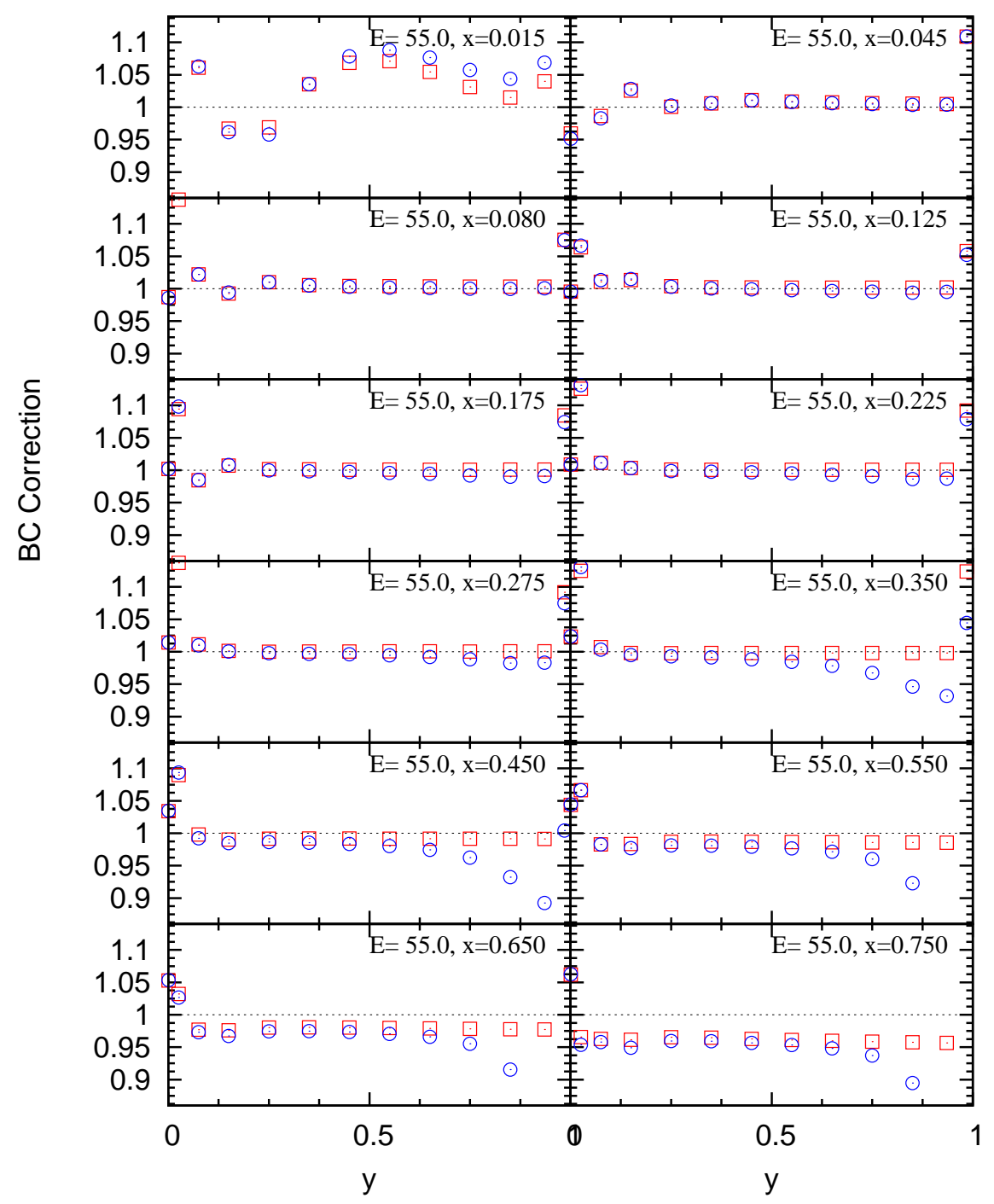

Bin Centering for Neutrinos Bin Centering for Anti-Neutrinos $\odot$

Figure 6.3: Bin centering correction for neutrinos and anti-neutrinos with $\mathrm{E}=55 \mathrm{GeV}$. 


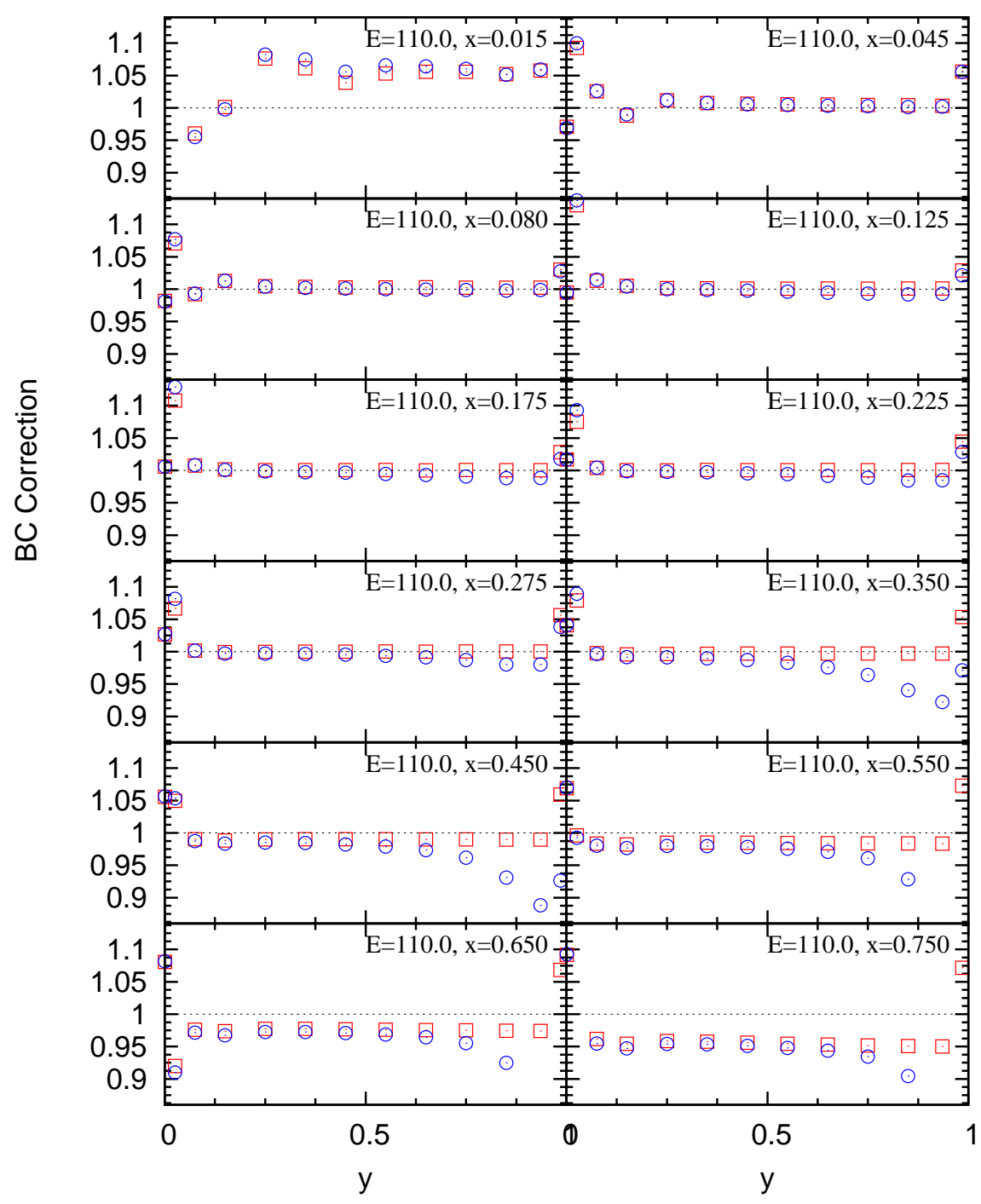

Bin Centering for Neutrinos Bin Centering for Anti-Neutrinos $\odot$

Figure 6.4: Bin centering correction for neutrinos anti-neutrinos with $\mathrm{E}=110 \mathrm{GeV}$. 


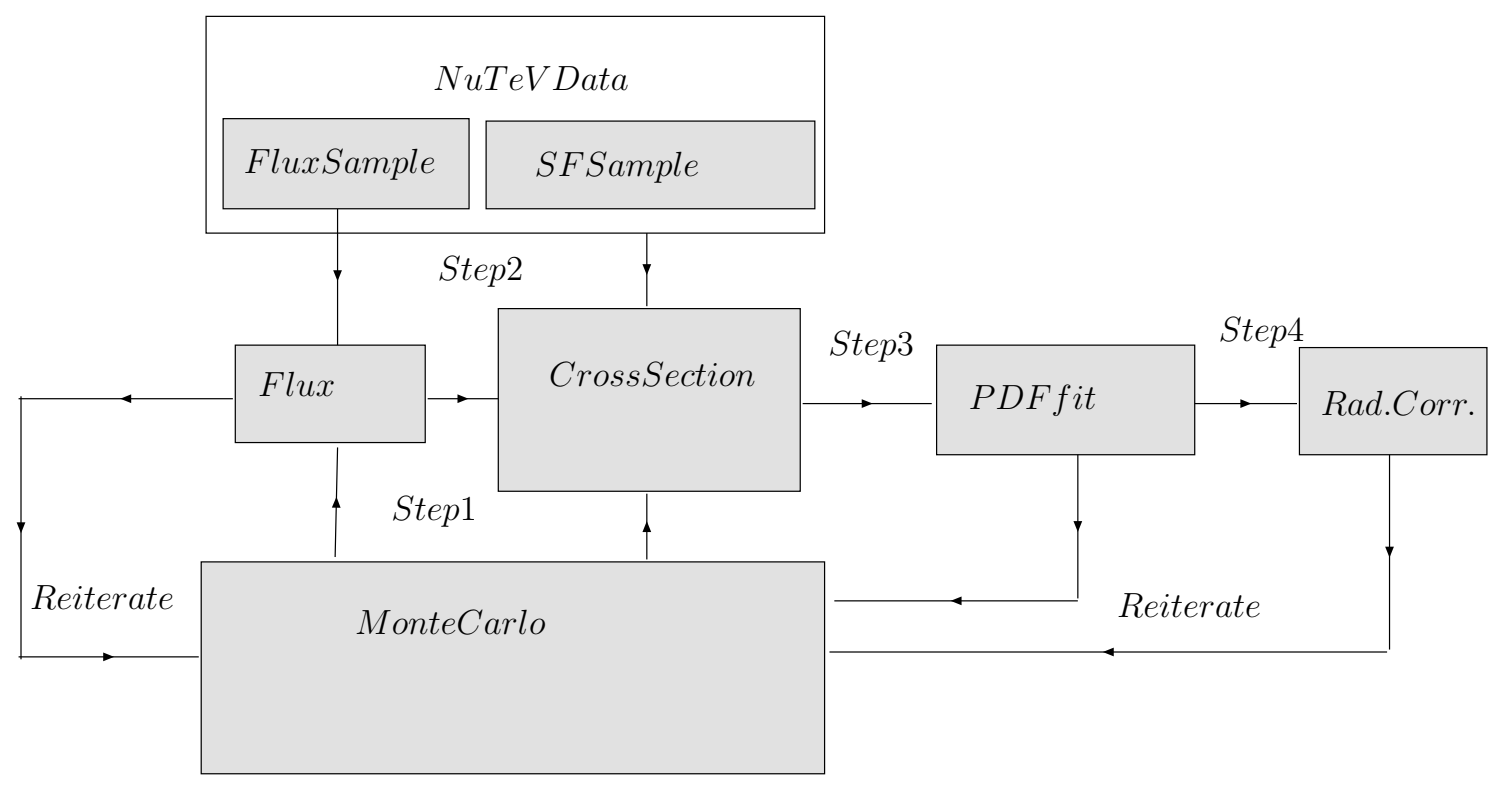

Figure 6.5: A schematic drawing of the iteration procedure used for cross section extraction.

This represents the differential cross section at the point $\left(x_{i}^{c}, y_{j}^{c}, E_{k}^{c}\right)$. There is small difference between the bin centering correction for neutrinos and anti-neutrinos, because the $y$ dependence of the cross sections differ.

\subsection{ITERATION PROCEDURE}

Neutrino flux and the cross section model are unknown a priori. An iterative procedure, (shown schematically in Figure 6.5), is used to obtain the final flux and differential cross section. For the "0-th" iteration an initial flux from beam Monte Carlo, and Buras-Gamers parameters from CCFR [2], are used to compute an acceptance correction to extract a "0-th" flux and " 0 -th" cross section. The iteration procedure is performed as follow:

- Step 1 - Generate acceptance corrections from NUMONTE using the cross section model extracted in the previous iteration. 
- Step 2 - Extract a flux and a differential cross section table using the acceptance corrections from Step 1.

- Step 3 - Perform a PDF fit to the differential cross section table from Step 2 (see Section 4.1.5).

- Step 4 - Compute the radiative corrections using the PDF parameters obtained in Step 3 and extract the new flux vertex distribution.

- Reiterate - Start over from Step 1

Iteration continues until the relative change in cross section value averaged over all data points from one iteration to the next is less than $0.1 \%$, which is achieved within three iterations. In the final iteration we require at least $20 \%$ acceptance for each kinematic bin, which excludes kinematic regions dominated by resolution smearing. A total of 2618 cross section bins are included in the final cross section table. The total number of bins for neutrinos is 1423 and for anti-neutrinos is 1195. The values of the final PDF parameters obtained from the fit to the final cross section table are given in Table 4.1. Figures 6.66.8 show the NuTeV final cross section and the final cross section model fit for $\mathrm{E}=55 \mathrm{GeV}$, $\mathrm{E}=110 \mathrm{GeV}$ and $\mathrm{E}=245 \mathrm{GeV}$ respectively in $x$ bins increasing from top to bottom as function of $y$ for neutrinos (left) and anti-neutrinos (right). The final goodness of fit $\chi^{2}$ is $\chi^{2} / d o f=$ $2225 / 2599$ including the systematic uncertainties. If we include in the $\chi^{2}$ calculation the full point-to-point covariance matrix discussed in Section 6.3 the quality of the fit worsens $\chi^{2}=3534 / 2599$. For comparison we computed the $\chi^{2}$ with two alternative external PDF parameterizations; $\chi^{2}=5969 / 2599$ using Bodek-Yang model [33] and $\chi^{2}=5000 / 2599$ using TR-VFS(MRST99) [34],[35]. Both alternative PDF parameterizations give poorer agreement with our data. Model dependence of the result was studied and appears to be small. Figure 6.9 shows a comparison between data and NUMONTE simulation using the final PDF fit parameters for the three physics quantities measured in $\mathrm{NuTeV}$ detector $E_{\mu}, E_{H A D}$ and $\theta_{\mu}$. NUMONTE models data well (at the $2 \%$ level). The yellow band shows the size of the systematic uncertainty. Highly precise charged lepton data are also included in the PDF fit (see Section 4.1.5). These data systematically pull the fit to agree with their $Q^{2}$ dependence, which worsens the quality of the fit with our data. 


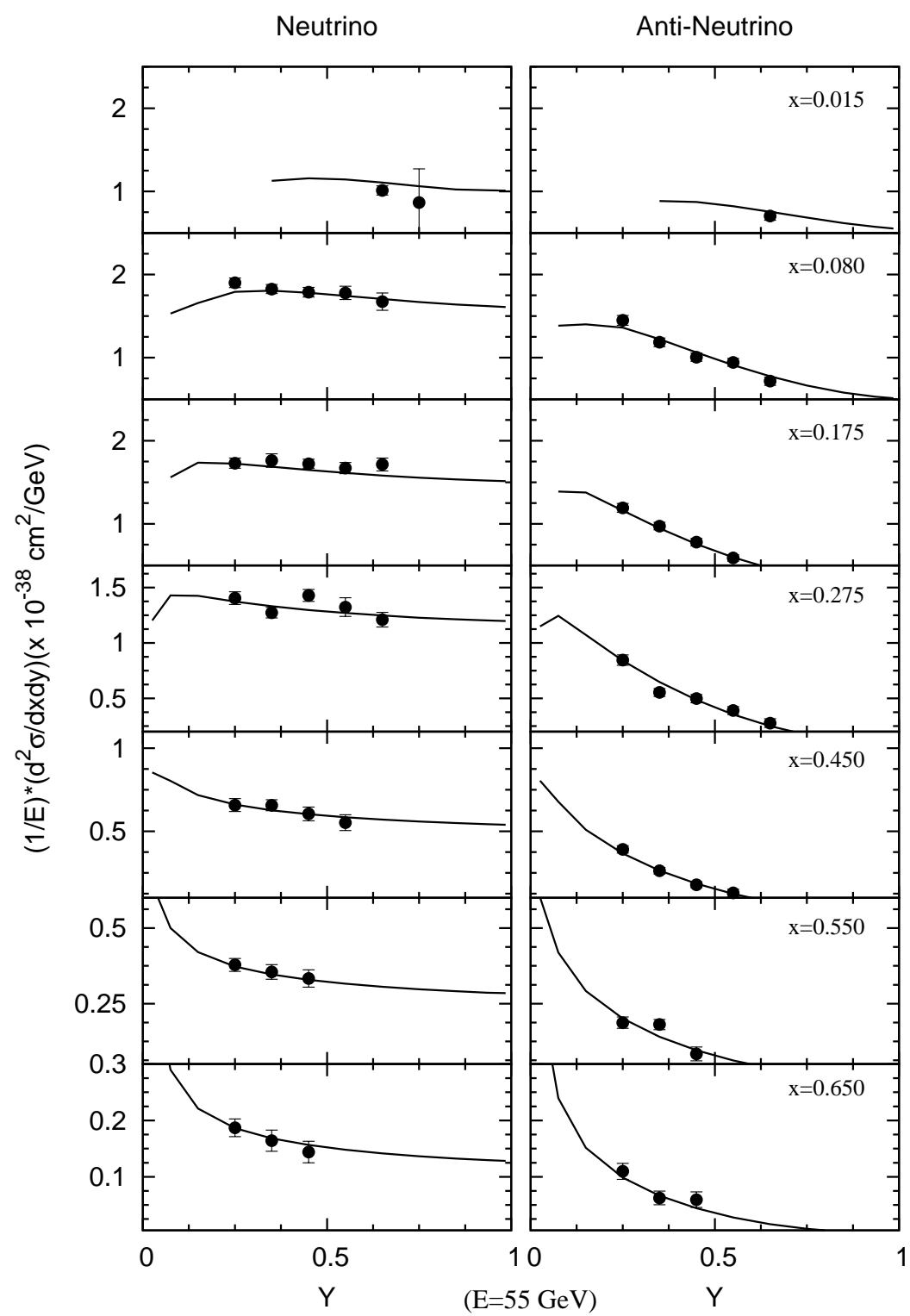

Figure 6.6: $\mathrm{NuTeV}$ final cross section and the final cross section model fit for $\mathrm{E}=55 \mathrm{GeV}$ in $x$ bins increasing from top to bottom as function of $y$ for neutrinos (left) and anti-neutrinos (right). 


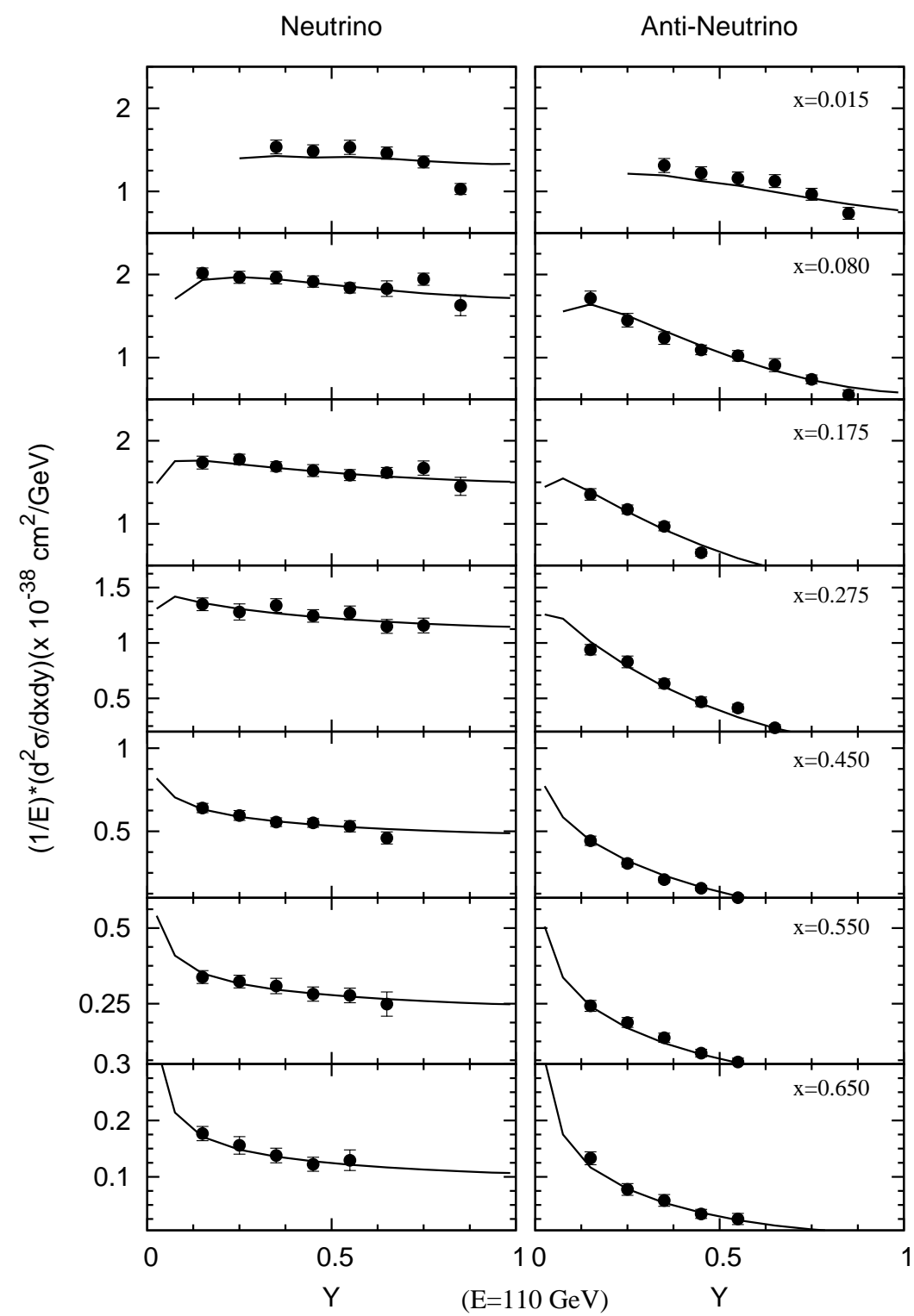

Figure 6.7: $\mathrm{NuTeV}$ final cross section and the final cross section model fit for $\mathrm{E}=110 \mathrm{GeV}$ in $x$ bins increasing from top to bottom as function of $y$ for neutrinos (left) and anti-neutrinos (right). 


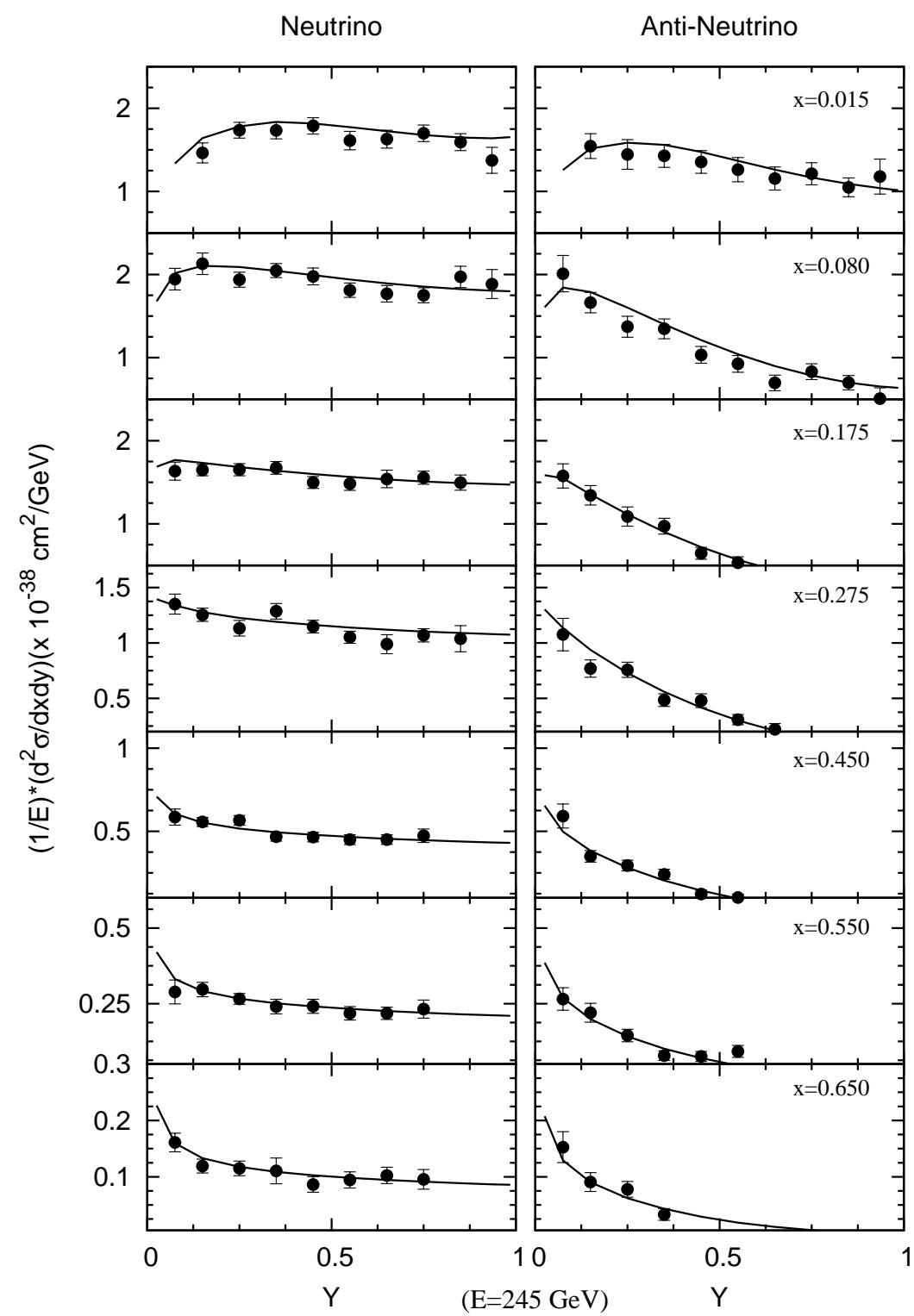

Figure 6.8: $\mathrm{NuTeV}$ final cross section and the final cross section model fit for $\mathrm{E}=245 \mathrm{GeV}$ in $x$ bins increasing from top to bottom as function of $y$ for neutrinos (left) and anti-neutrinos (right). 

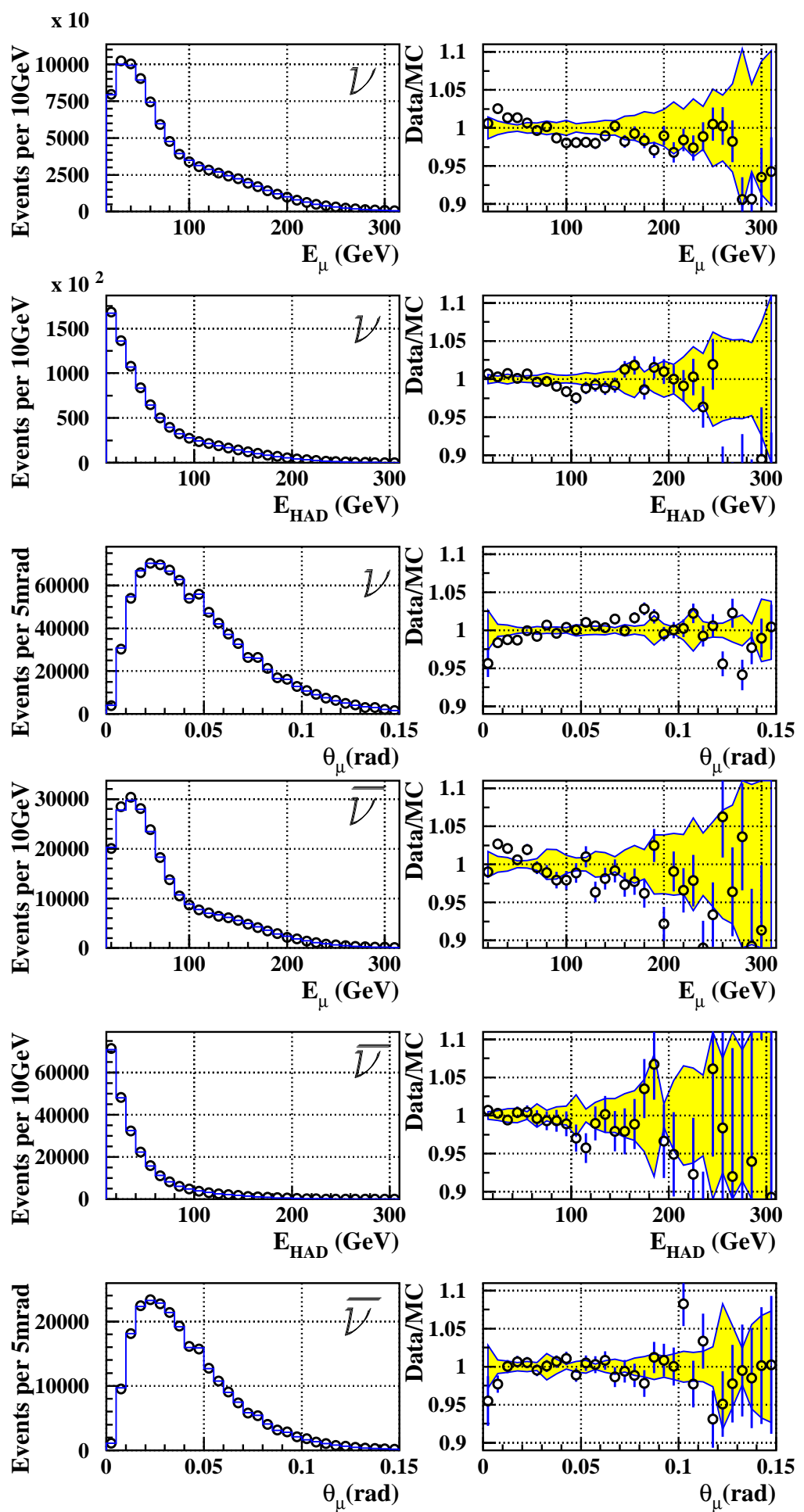

Figure 6.9: Monte Carlo compared with data for the muon energy, the hadron energy and muon angle for neutrinos(top three) and anti-neutrinos (bottom three). The yellow band represents the systematic uncertainty. 


\subsection{SYSTEMATIC UNCERTAINTIES}

There are 7 systematic uncertainties found to contribute to the total uncertainty; the muon and hadron energy scales, charm mass $m_{c}, B / A$ fit value, muon and hadron energy smearing models, and the flux normalization. All of these uncertainties were described in Section 5.2.1. Each uncertainty is evaluated separately by propagating it through the whole cross section extraction procedure. The contribution from each systematic uncertainty (except muon energy smearing) is evaluated by shifting the central value of the systematic parameter by $\pm \sigma$. Cross sections are re-extracted for both shifts and the symmetrized difference in each cross section point is taken to be the systematic uncertainty due to this parameter. The muon energy smearing uncertainty is evaluated by comparing the default model (Equation 3.20) with an alternative parameterization of the leading Gaussian (Equation 3.21). The cross section is re-extracted with this model. The difference from the default, for each cross section point, is taken to be the systematic uncertainty due to the muon energy smearing model.

The muon and hadron energy scale uncertainties, which give the largest contribution to the systematic uncertainty, affect both the flux and the cross section extraction. The $2.1 \%$ flux normalization uncertainty (see Section 5.3) is treated as an overall normalization uncertainty in the cross section.

We evaluated the cross section model uncertainty using fit parameter errors. New acceptance corrections were generated using a model which was obtained from the default by shifting up or down each parameter by its fit error. Flux and cross section were extracted for each fit parameter shift. The size of the systematic uncertainty in the cross section due to each fit parameter was taken to be the symmetrized difference of the cross sections. This contribution to the uncertainty was neglected because the resultant shifts in the acceptance corrections were consistent with statistical fluctuations. (The Monte Carlo samples were generated with 20 times the statistics of data).

A point-to-point covariance matrix is calculated to account for the correlations between 
data points. The full covariance matrix $M_{\alpha \beta}$ is given by

$$
M_{\alpha \beta}=\sum_{i}^{7} \delta_{i \mid \alpha} \delta_{i \mid \beta}
$$

where $\delta_{i \mid \alpha}$ is the $1 \sigma$ shift in data point $\alpha$ due to systematic uncertainty $i$. The $2.1 \%$ flux normalization uncertainty can be included in the covariance matrix by adding a term

$$
M_{\alpha \beta}^{\prime}=M_{\alpha \beta}+(0.021)^{2}{\frac{d^{2} \sigma}{d x d y}}_{\alpha}^{D}{\frac{d^{2} \sigma}{d x d y}}_{\beta}^{D}
$$

The statistical uncertainty is added in quadrature to the diagonal elements of the data covariance matrix. Previous cross section measurements from reference [1] and [2] did not provide such data correlation matrix.

Separate data vectors and covariance matrices are obtained for the neutrino and antineutrino cross sections. A $\chi^{2}$ with respect to a theoretical model can be calculated using

$$
\begin{aligned}
& \chi^{2}= \\
& =\sum_{\alpha, \beta=1}^{N_{D A T A}^{\nu}}\left[{\frac{d^{2} \sigma^{t h}}{d x d y_{\alpha}}}^{t h}-{\frac{d^{2} \sigma}{d x d y}}_{\alpha}^{D}\right]\left(\mathbf{M}_{\nu}^{-1}\right)_{\alpha \beta}\left[{\frac{d^{2} \sigma}{d x d y_{\beta}}}^{t h}-{\frac{d^{2} \sigma}{d x d y_{\beta}}}^{D}\right]
\end{aligned}
$$

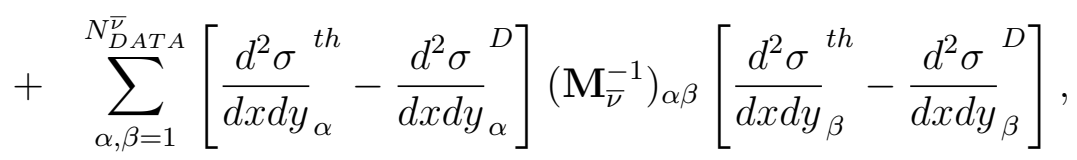

where ${\frac{d^{2} \sigma}{d x d y}}_{\alpha}^{D}$ is the measured differential cross section and ${\frac{d^{2} \sigma}{d x d y}}_{\alpha}^{\text {th }}$ is the model prediction for data point $\alpha$. 


\subsection{RESULTS AND COMPARISON WITH CCFR AND CDHSW}

Figures 6.10 - 6.12 show the extracted differential cross section for three energy bins $\mathrm{E}=65 \mathrm{GeV}$, $\mathrm{E}=150 \mathrm{GeV}$ and $\mathrm{E}=245 \mathrm{GeV}$. The plot shows $x$ bins increasing from top to bottom $(x=0.08$, $0.225,0.45$, and 0.75 bins are not shown) as function of y for neutrinos (left) and antineutrinos (right). Our data are compared with two previous measurements by the CDHSW (CERN-Dortmund-Heidelberg-Saclay-Warsaw) collaboration [1] and the CCFR collaboration $[2]$.

CDHSW used a detector for which the entire iron was magnetized. They used combined neutrino and anti-neutrino beam with energy in the range $20<E<212 \mathrm{GeV}$. Their data sample contained 640,000 neutrino and 550,000 anti-neutrino events. CCFR used combined neutrino and anti-neutrino beam with energy in the range $30<E<360 \mathrm{GeV}$. Their final data sample contained 1,030,000 neutrino and 179,000 anti-neutrino events. Both previous experiments used detectors similar to the $\mathrm{NuTeV}$ detector and had data samples which are statistically comparable and have similar energy range.

Our result is in reasonable agreement with CDHSW (except perhaps at lowest $\mathrm{x}$ ). The CDHSW measurement has a known $y$ shape difference with the CCFR, and thus also with this result. However, it is difficult to perform a direct comparison between $\mathrm{NuTeV}$ and CDHSW data, because CDHSW cross section data points represent the average cross section for the bin (not bin-centered).

Our result agrees well with CCFR for $x<0.4$ over the full energy and $y$ range of the data, both in level and in shape. $\mathrm{NuTeV}$ and CCFR differential cross sections differ at $x>0.40$ where CCFR's measurement for both neutrino and antineutrino cross sections are consistently below our result for all energies. Figure 6.13 shows the weighted average over E and y of the point-to-point ratio of CCFR and $\mathrm{NuTeV}$ cross sections in $x$ bins for neutrinos (top) and anti-neutrinos(bottom). The level difference in the cross sections at high $x$ is constant over the full $y$ range of the data. The difference in the neutrino cross sections are $4 \pm 1 \%$ at $x=0.45,9 \pm 2 \%$ at $x=0.55$, and increases with $x$ up to $18 \pm 2 \%$ at $x=0.65$, (and similarly for antineutrinos). CCFR did not report data above $x=0.7$, because the muon and hadron energy smearing were not well modeled there. 


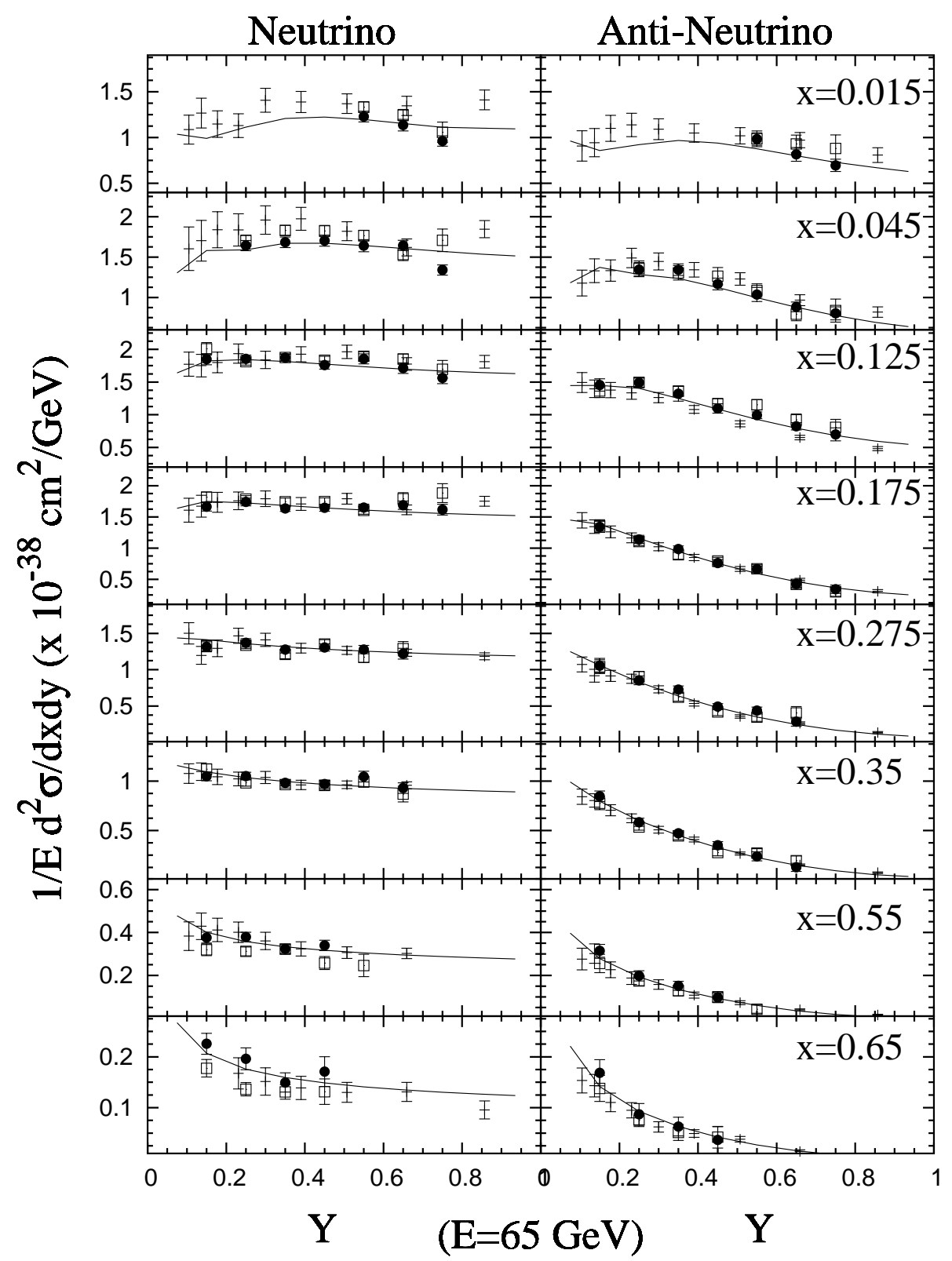

Figure 6.10: Differential cross sections in $x$ bins for neutrinos (left) and anti-neutrinos (right) at $E=65 \mathrm{GeV}$. Points are $\mathrm{NuTeV}$ (filled circles), CCFR (open squares), and CDHSW (crosses). Error bars show statistical and systematic errors in quadrature. Solid curve shows fit to $\mathrm{NuTeV}$ data. ( $x=0.08,0.225,0.45$, and 0.75 bins are not shown). 


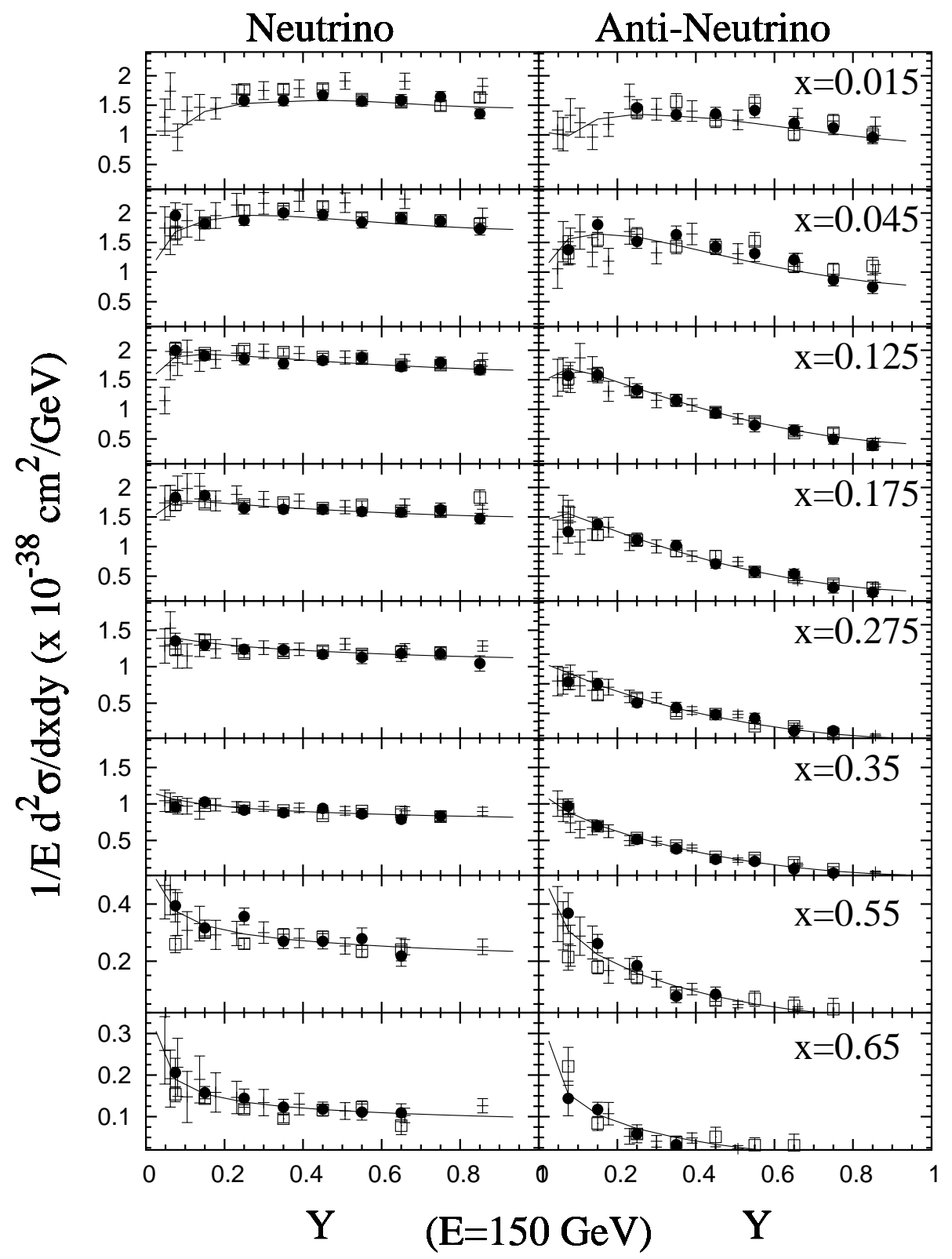

Figure 6.11: Differential cross sections in $x$ bins for neutrinos (left) and anti-neutrinos (right) at $E=150 \mathrm{GeV}$. Points are NuTeV (filled circles), CCFR (open squares), and CDHSW (crosses). Error bars show statistical and systematic errors in quadrature. Solid curve shows fit to $\mathrm{NuTeV}$ data. ( $x=0.08,0.225,0.45$, and 0.75 bins are not shown). 


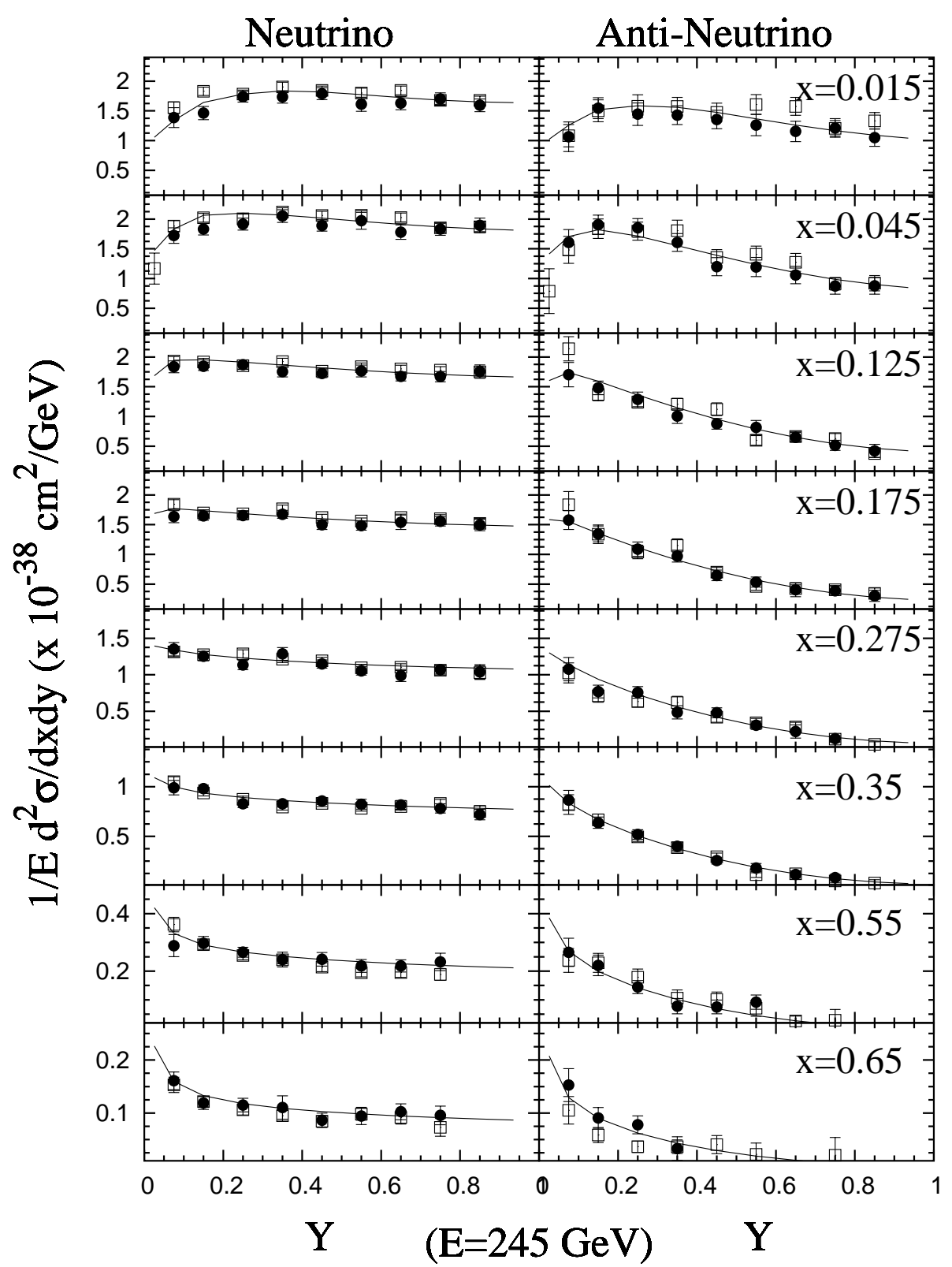

Figure 6.12: Differential cross sections in $x$ bins for neutrinos (left) and anti-neutrinos (right) at $E=245 \mathrm{GeV}$. Points are $\mathrm{NuTeV}$ (filled circles), CCFR (open squares). (CDHSW does not have data above $190 \mathrm{GeV}$ ). Error bars show statistical and systematic errors added in quadrature. Solid curve shows fit to $\mathrm{NuTeV}$ data. $(x=0.08,0.225,0.45$, and 0.75 bins are not shown). 

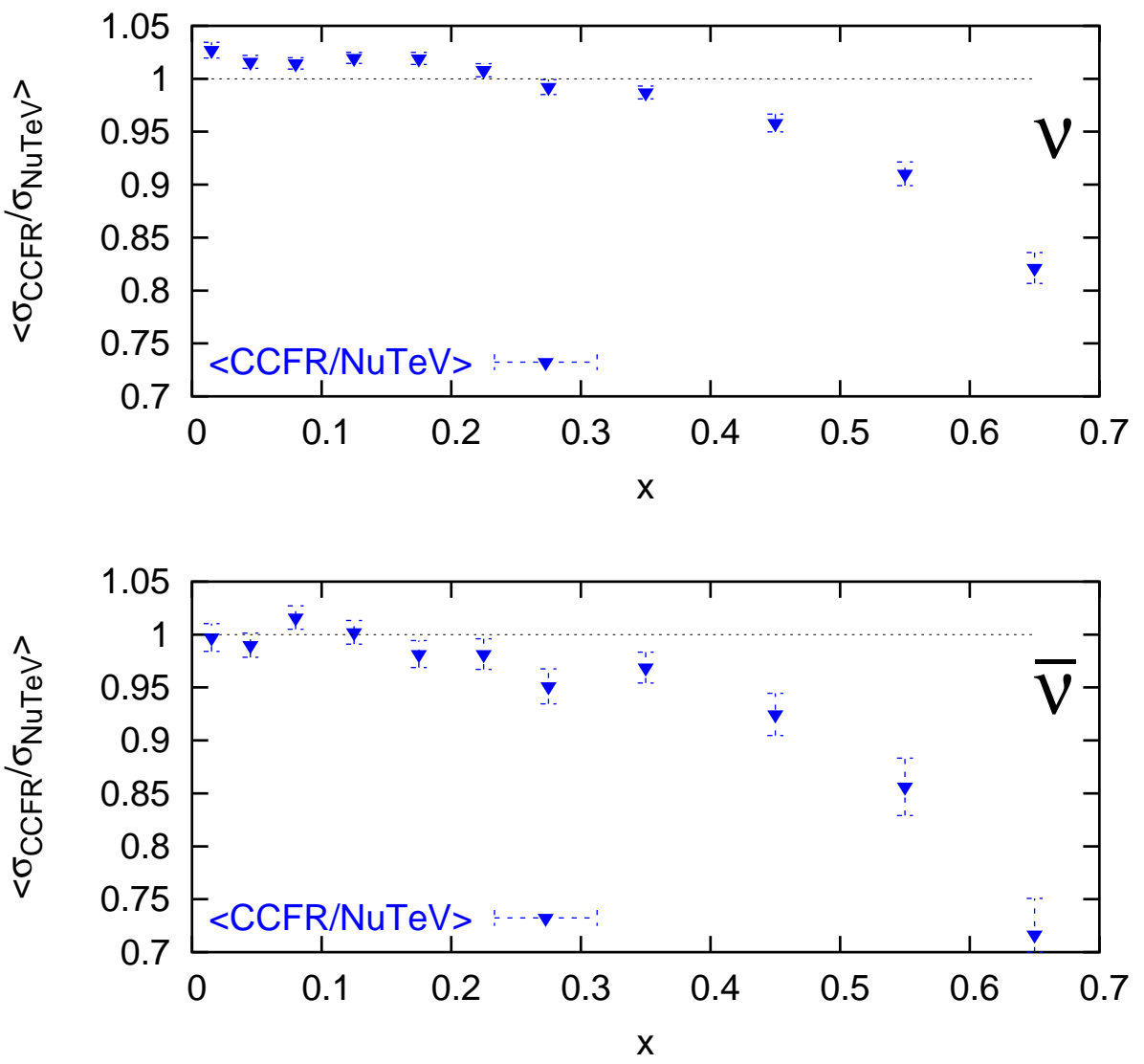

Figure 6.13: Point-to-point ratio of CCFR and NuTeV cross sections weight averaged over $\mathrm{E}$ and $\mathrm{y}$ as function of $\mathrm{x}$ for neutrinos (top) and anti-neutrinos(bottom). 


\subsection{NUTEV-CCFR HIGH X DISCREPANCY}

$\mathrm{NuTeV}$ and CCFR used similar detectors and analysis techniques, thus the difference in the results is suprising. In fact, $\mathrm{NuTeV}$ used the refurbished CCFR detector. The scintillator oil was replaced and the drift chambers were reconditioned. The precise calibration beam allowed $\mathrm{NuTeV}$ to understand better the response of the detector. Differences in analysis method and calibration differences were studied to find the source of the discrepancy.

The largest single contribution to the discrepancy between NuTeV and CCFR is found to be due to the model of the magnetic field of the toroid used. Both CCFR and NuTeV used the same magnet to analyze the muon momenta. The magnitude of the magnetic field depends on the current and the total number of turns of the coils, but the radial dependence depends only on the geometry of the toroid. Therefore the radial dependence of the magnetic field for both CCFR and NuTeV models should be the same, while the magnitude of the field could be different. In principle, the magnitude of the field could be different due to difference in the actual current in the coils. To make a meaningful comparison one can choose a location on the face of the toroid for which both magnetic fields are known with high certainty.

$\mathrm{NuTeV}$ used ANSYS to compute the magnetic field map and compared the prediction to precision test beam mapped data (see Section 3.3.3). NuTeV test beam mapped the entire toroid with continuous high statistics $50 \mathrm{GeV}$ muon map runs, which provided calibration points for both the absolute muon energy scale and the radial dependence of the magnetic field (see Section 3.3.3). CCFR used POISSON [36] to model the magnetic field map. CCFR calibration beam data were taken twice [37]; in a series of low statistics runs with various energies and locations during E744, and at the end of E770 in one high statistics run at one location on the toroid's face. The second high statistics run was used to set the overall muon energy scale. The event by event precision of CCFR test beam was 1.0\%. Figure 6.14 shows the ratio of the magnetic field maps. The value of the ratio is adjusted to be 1.0 at the region where both experiments have high statistics calibration beam data. The difference between the magnetic field models ranges from $-6 \%$ near the hole to $3 \%$ at the edge of the detector. The average of the ratio in Figure 6.14 over the working surface of the toroid is 


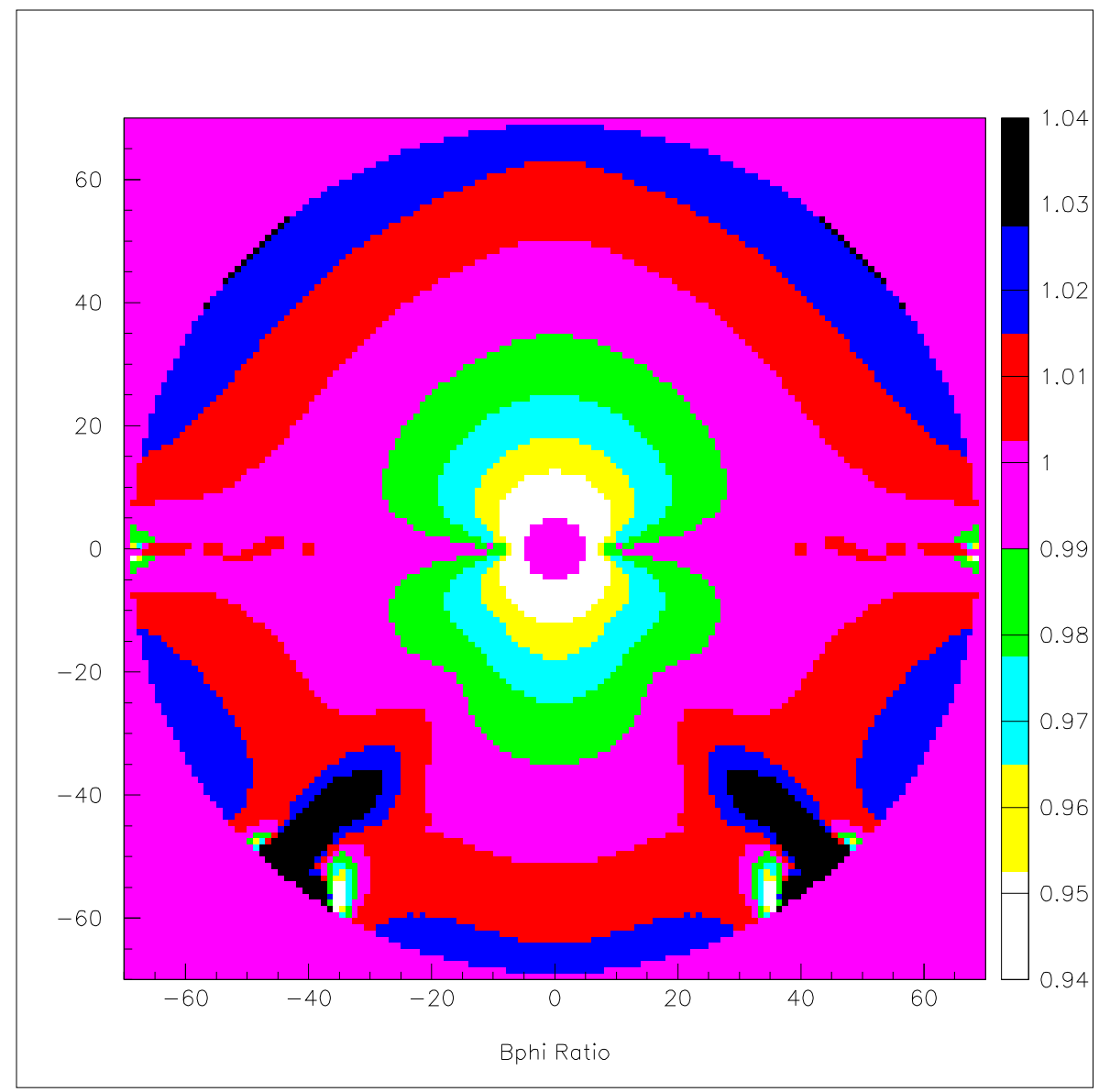

Figure 6.14: Ratio of CCFR and NuTeV magnetic field maps as function of the (x,y) position. The ratio is adjusted to have value 1.0 at the position where both $\mathrm{NuTeV}$ and CCFR have high precision calibration beams. 
0.992, which corresponds to an effective $0.8 \%$ shift down in the muon energy scale. $\mathrm{NuTeV}$ data was processed with CCFR magnetic field map and the cross section was extracted. This difference accounts for $6 \%$ of the $18 \%$ difference at $x=0.65$.

Additional, small sources of the discrepancy were studied. Both $\mathrm{NuTeV}$ and CCFR use an iterative fit to determine the acceptance corrections. As a result the cross section models are different, because they fit the corresponding data set. Therefore at $x=0.65, \mathrm{NuTeV}$ cross section model is $20 \%$ higher than the model used by CCFR. NuTeV flux and cross section were extracted using the CCFR cross section model. The difference between the cross section extracted with the CCFR and the NuTeV cross section models accounts for $3 \%$ of the $18 \%$ discrepancy at $x=0.65$. The different muon and hadron energies smearing models were also studied and the cross section was extracted using the CCFR smearing models to generate acceptance corrections. The effect of smearing model differences is $2 \%$ at $x=0.65$. Hadron energy response of $\mathrm{NuTeV}$ calorimeter was found to have a small nonlinearity due to the ranging with energy $\pi^{0}$ fraction in the shower [15]. This non-linearity was taken into account in $\mathrm{NuTeV}$ but not in CCFR. The effect of incorporating the hadron energy scale nonlinearity is small and contributes mainly to the $Q^{2}$ dependence.

All together these studied contributions account for about two thirds of the high- $x$ cross section difference seen. This brings the two measurements within 1.2 sigma agreement at $x=0.65$.

Another difference results from the different beam running modes of $\mathrm{NuTeV}$ and CCFR. $\mathrm{NuTeV}$ used high purity separate neutrino and anti-neutrino beams (see Section 2.1.2), which allowed the toroid polarity to be set to focus $\mu^{-}$, in neutrino mode, and $\mu^{+}$, in anti-neutrino mode. CCFR used a beam with an 11\% anti-neutrino component and took neutrino and anti-neutrino data simultaneously. The polarity of the toroidal magnetic field was reversed periodically (50\% running in each toroid mode) to obtain high statistics for anti-neutrino interactions. As a result, CCFR had four different samples; focusing and defocusing for both neutrino and anti-neutrino data. Detector acceptance is better in focusing mode compared to defocusing mode, because in the latter the muons are bent out of the toroid resulting in a shorter track. The detector has poorer acceptance in the high-x and high-y kinematic region, especially in defocusing mode since large angle muons spend less time in the toroid. To study 
the effect of this we would need to separate CCFR data into four samples. Unfortunately, there is not enough information to quantify the effect of the difference on the cross section measurement further. 


\subsection{STRUCTURE FUNCTIONS}

\subsection{STRUCTURE FUNCTION FROM ONE PARAMETER FITS}

The structure functions can be measured from linear combinations of the neutrino and antineutrino cross sections. The sum of the differential cross sections gives

$$
\begin{aligned}
\frac{d^{2} \sigma^{\nu}}{d x d y} & +\frac{d^{2} \sigma^{\bar{\nu}}}{d x d y} \\
& =\frac{G_{F}^{2} M E}{\pi}\left[\left(1-y-\frac{M x y}{2 E}+\frac{y^{2}}{2} \frac{1+4 M^{2} x^{2} / Q^{2}}{1+R_{L}}\right) 2 \overline{F_{2}}+y\left(1-\frac{y}{2}\right) \Delta x F_{3}\right]
\end{aligned}
$$

where $2 \overline{F_{2}}=F_{2}^{\nu}+F_{2}^{\bar{\nu}}$ and $\Delta x F_{3}=x F_{3}^{\nu}-x F_{3}^{\bar{\nu}}$. Equation 7.1 is obtained from the 'bare' cross section for neutrino scattering off an isoscalar target. The cross section table extracted in the previous chapter is the differential cross section for scattering off an iron target, which is a heavy nuclear target with small non-isoscalarity. To extract the structure functions we correct the measured cross section for radiative effects, massive propagator and for nonisoscalarity. We apply the multiplicative correction

$$
C_{\text {Bare }}(x, y, E)=\frac{\frac{d^{2} \sigma}{d x d y}\left(\text { Iso, } M_{W}=0, \text { NoRad.Corr }\right)(x, y, E)}{\frac{d^{2} \sigma}{d x d y}\left(F e, M_{W}=80.4 G e V, \text { Rad.Corr }\right)(x, y, E)},
$$

which is obtained from our Monte Carlo cross section model, to each cross section point. We re-bin the cross section from $\left(x_{c}, y_{c}, E_{c}\right) \rightarrow\left(x_{c}, y_{c}, Q_{c}^{2}\right)$ to express the structure functions as functions of $x$ and $Q^{2}$. The limits and centers of the $Q^{2}$ bins are given in table 7.1. In general, the $Q^{2}$ of the rebinned data points do not coincide with the bin centers, $Q_{c}^{2}$. A bin centering correction is applied to each new $Q^{2}$ point to move it to the bin center

$$
C_{Q_{c}^{2}}=\frac{\frac{d^{2} \sigma}{d x d y}\left(x, y, Q_{c}^{2}\right)}{\frac{d^{2} \sigma}{d x d y}\left(x, y, Q^{2}=2 M x y E\right)}
$$




\begin{tabular}{|c|c|c|}
\hline$Q^{2}$ bin & Bin limits $\log _{10} Q^{2} \mathrm{GeV}^{2} / c^{2}$ & Bin centers $\log _{10} Q^{2} \mathrm{GeV}^{2} / c^{2}$ \\
\hline 1 & $0-0.2$ & 0.1 \\
\hline 2 & $0.2-0.4$ & 0.3 \\
\hline 3 & $0.4-0.6$ & 0.5 \\
\hline 4 & $0.6-0.8$ & 0.7 \\
\hline 5 & $0.8-1.0$ & 0.9 \\
\hline 6 & $1.0-1.2$ & 1.1 \\
\hline 7 & $1.2-1.4$ & 1.3 \\
\hline 8 & $1.4-1.6$ & 1.5 \\
\hline 9 & $1.6-1.8$ & 1.7 \\
\hline 10 & $1.8-2.0$ & 1.9 \\
\hline 11 & $2.0-2.2$ & 2.1 \\
\hline 12 & $2.2-2.4$ & 2.3 \\
\hline 13 & $2.4-2.6$ & 2.5 \\
\hline 14 & $2.6-2.8$ & 2.7 \\
\hline
\end{tabular}

Table 7.1: $Q^{2}$ bin limits and centers given in $\log _{10} Q^{2}(\mathrm{GeV})$. 
After correcting all energy bins entering a given $Q^{2}$ bin using (Equation 7.3) a weighted average is computed to obtain the value of the cross section in bin $\left(x_{c}, y_{c}, Q_{c}^{2}\right)$.

The structure functions $\bar{F}_{2}, R_{L}$ and $\Delta x F_{3}$ can be determined from the y-dependence of the cross section sum, using equation 7.1. However, due to large correlations between $R_{L}$ and $\Delta x F_{3}$ at low $x$ it is important that our data cover a large range in $y$. Detector acceptance for this toroid analyzed muon sample is limited in the the low-x and high-y kinematic region. Cross section data from a future target muon sample will provide additional data points in the low-x and high-y kinematic region. In this analysis we present a 1-parameter fit to the $y$-dependence to determine the structure function $\bar{F}_{2}$, which requires models for $R_{L}$ and $\Delta x F_{3}$ as inputs. The input model for $R_{L}$ is obtained from a parameterization of world data from reference [28], given by Equation 4.14. If we assume charm and strange sea symmetry, in leading order $\Delta x F_{3}=4(s-c)$. For $x<0.1$ we use TRVFS(MRST99) [34, 35] prediction for $\Delta x F_{3}$, while for $x>0.1 \Delta x F_{3}$ is negligible.

The difference of the cross sections gives

$$
\begin{aligned}
\frac{d^{2} \sigma^{\nu}}{d x d y} & -\frac{d^{2} \sigma^{\bar{\nu}}}{d x d y} \\
& =\frac{G_{F}^{2} M E}{\pi}\left[\left(1-y-\frac{M x y}{2 E}+\frac{y^{2}}{2} \frac{1+4 M^{2} x^{2} / Q^{2}}{1+R_{L}}\right) \Delta F_{2}+y\left(1-\frac{y}{2}\right) 2 x \bar{F}_{3}\right]
\end{aligned}
$$

where $\Delta F_{2}=F_{2}^{\nu}-F_{2}^{\bar{\nu}}$ and $2 x \bar{F}_{3}=x F_{3}^{\nu}+x F_{3}^{\bar{\nu}}$. The term $\Delta F_{2}$ is very small and is neglected. $x \bar{F}_{3}$ is determined from a 1-parameter fit to the y-dependence of the difference of the neutrino and anti-neutrino cross sections.

Figures 7.1 and 7.2 show the extracted $\bar{F}_{2}$ and $x \bar{F}_{3}$ structure functions for neutrino scattering off an isoscalar iron target. Both structure functions are compared with previous neutrino iron measurements: $F_{2}$ is compared with CCFR [2] and CDHSW [1], and $x F_{3}$ with CCFR97 [32] and CDHSW. Both statistical and systematic uncertainties are added in quadrature and shown in the plots.

The systematic uncertainty for the $\mathrm{NuTeV}$ structure functions is obtained by propagating the uncertainty of the differential cross section (see Section 6.3). For the $\bar{F}_{2}$ measurement, we evaluated the additional contributions to the systematic uncertainty due to the input models for $R_{L}$ and $\Delta x F_{3}$. The 1- $\sigma$ uncertainty in the $R_{L}$ parameterization to the world data 


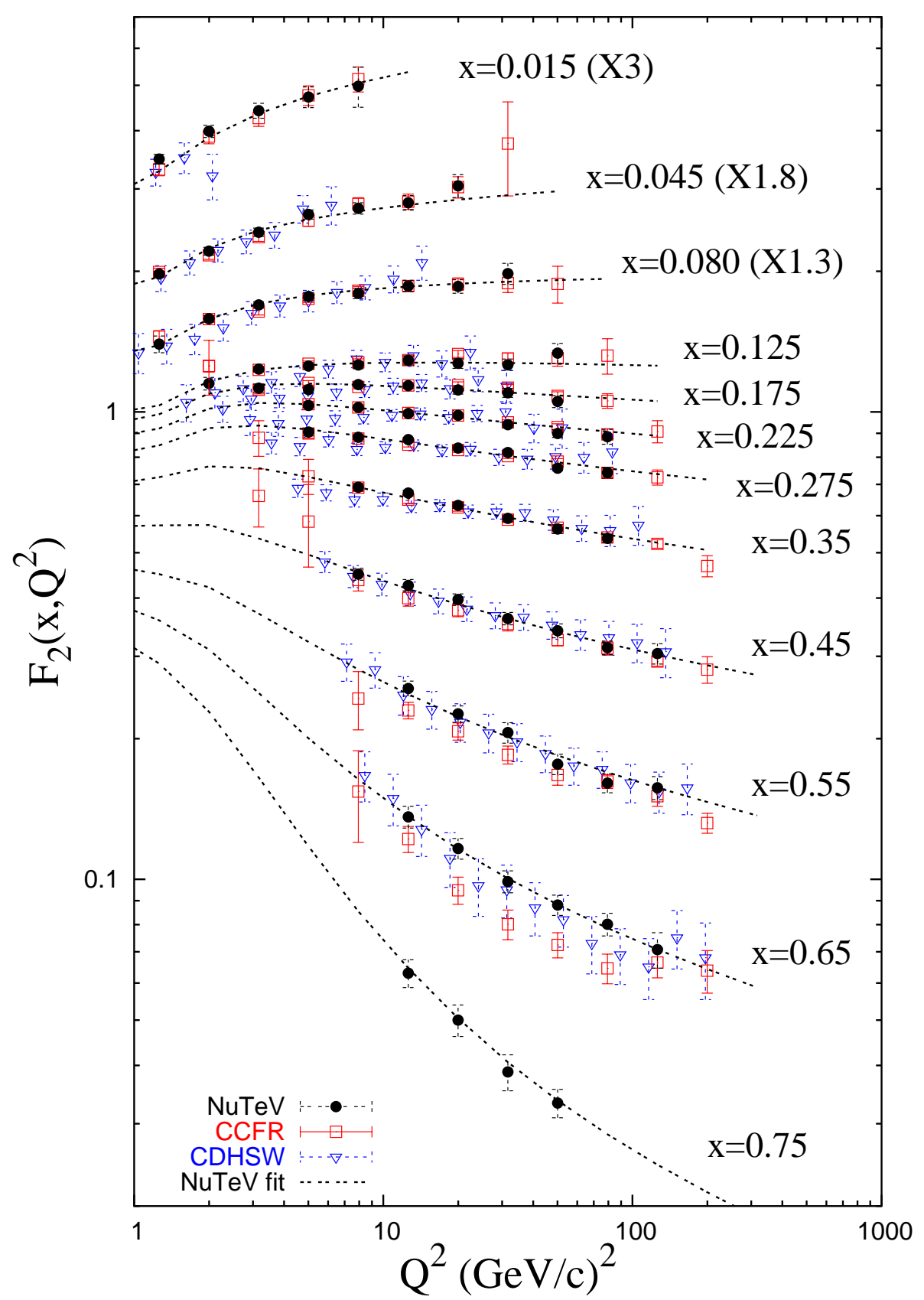

Figure 7.1: NuTeV measurement of $F_{2}\left(x, Q^{2}\right)$ (solid circles) compared with previous $\nu$-Fe results; CCFR (open circles) and CDHSW (triangles). The data are corrected to an isoscalar (iron) target and for QED radiative effects as described in the text. The curve show the $\mathrm{NuTeV}$ model. 


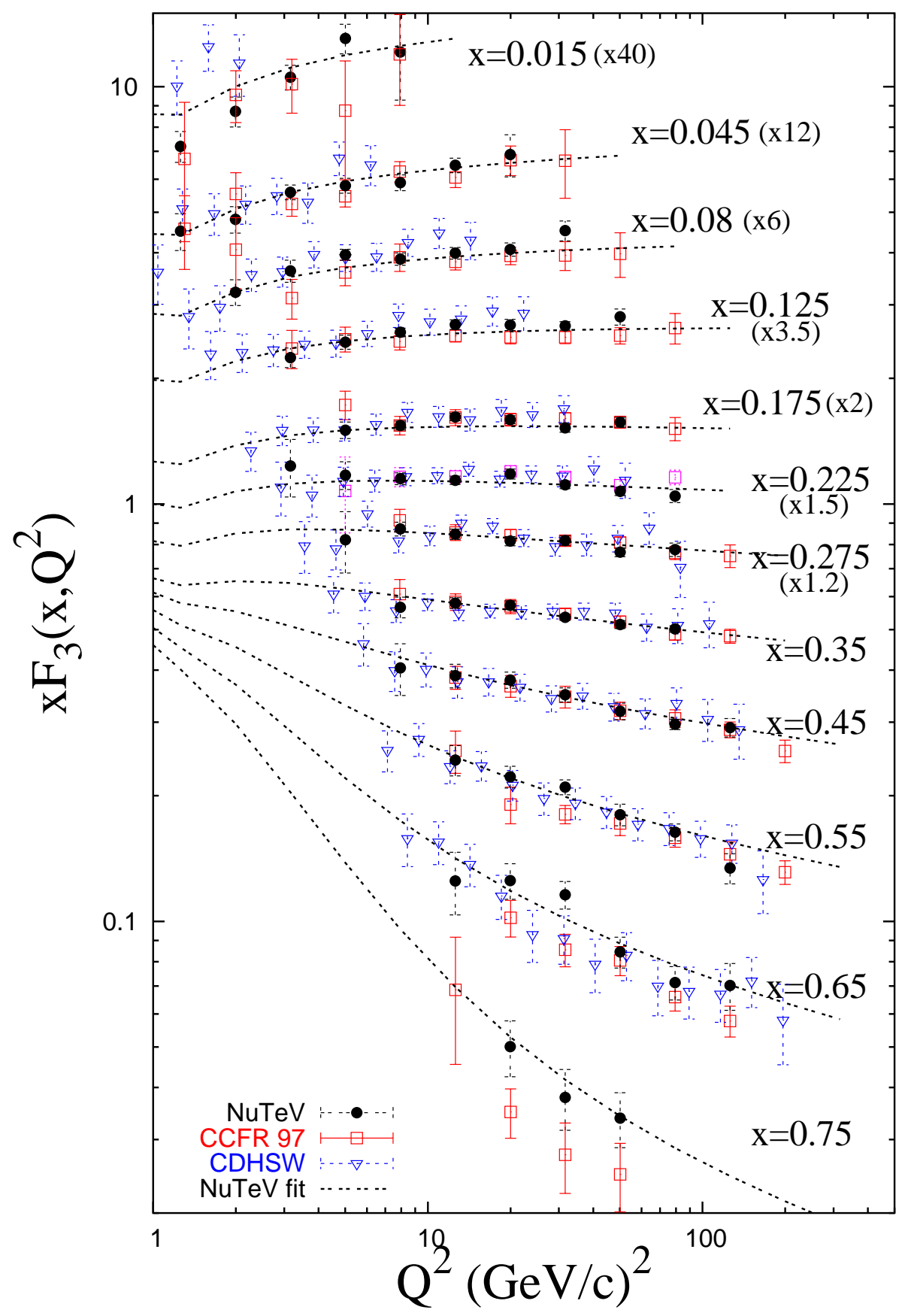

Figure 7.2: NuTeV measurement of $x F_{3}\left(x, Q^{2}\right)$ (solid circles) compared with previous $\nu$-Fe results; CCFR (open circles) and CDHSW (triangles). The data are corrected to an isoscalar (iron) target and for QED radiative effects as described in the text. The curve show the $\mathrm{NuTeV}$ model. 
is $15 \%$. New $F_{2}$ fits were obtained by shifting the value of $R_{L}$ by $\pm \sigma$ and the symmetrized difference for each $F_{2}$ point was taken to be the systematic uncertainty from the $R_{L}$ model. An alternative model for $\Delta x F_{3}$ is used to evaluate the contribution to the uncertainty. The difference between the $F_{2}$ value obtained with the default (TRVFS) and the alternative (ACOTFFS) model gives the $\Delta x F_{3}$ model systematic uncertainty.

Within errors, our result agrees in level with CDHSW but not in shape, as is the case for the differential cross section. At $x<0.4$, this result agrees well with CCFR over the $x$ and $Q^{2}$ range of the data, both in level and in shape. NuTeV structure functions for at $x>0.4$ are consistently above CCFR's for all $Q^{2}$ values. This propagates directly from the difference in the cross sections discussed in Section 6.5. The results will be compared with theory models in Section 7.3.

\subsection{STRUCTURE FUNCTIONS FROM TWO PARAMETER FIT}

In order to use both $F_{2}$ and $x F_{3}$ together in a QCD analysis one needs the correlation between them. A simultaneous fit to the $y$ dependence of the neutrino and anti-neutrino cross sections is performed to obtain both $\bar{F}_{2}, x \bar{F}_{3}$ and their correlation using the equations

$$
\begin{aligned}
\frac{d^{2} \sigma^{\nu}}{d x d y} & =\frac{G_{F}^{2} M E}{\pi}\left[\left(1-y-\frac{M x y}{2 E}\right.\right. \\
& \left.\left.+\frac{y^{2}}{2} \frac{1+4 M^{2} x^{2} / Q^{2}}{1+R_{L}}\right)\left(\bar{F}_{2}+\frac{\Delta F_{2}}{2}\right)+y\left(1-\frac{y}{2}\right)\left(x \bar{F}_{3}+\frac{\Delta x F_{3}}{2}\right)\right], \\
\frac{d^{2} \sigma^{\bar{\nu}}}{d x d y} & =\frac{G_{F}^{2} M E}{\pi}\left[\left(1-y-\frac{M x y}{2 E}\right.\right. \\
& \left.\left.+\frac{y^{2}}{2} \frac{1+4 M^{2} x^{2} / Q^{2}}{1+R_{L}}\right)\left(\bar{F}_{2}-\frac{\Delta F_{2}}{2}\right)+y\left(1-\frac{y}{2}\right)\left(x \bar{F}_{3}-\frac{\Delta x F_{3}}{2}\right)\right],
\end{aligned}
$$

where $2 \overline{F_{2}}=F_{2}^{\nu}+F_{2}^{\bar{\nu}}, \Delta F_{2}=F_{2}^{\nu}-F_{2}^{\bar{\nu}}, 2 x \bar{F}_{3}=x F_{3}^{\nu}+x F_{3}^{\bar{\nu}}$ and $\Delta x F_{3}=x F_{3}^{\nu}-x F_{3}^{\bar{\nu}}$. As described in the previous section $\Delta F_{2}$ is small and is therefore neglected. $R_{L}$ and $\Delta x F_{3}$, as before, are used as input. The cross section points are rebinned using the same procedure described in the previous section. The combined $\chi^{2}$

$$
\chi^{2}=\chi_{\nu}^{2}+\chi_{\bar{\nu}}^{2}
$$


which is constructed using both Equations 7.5 and 7.6 is minimized. The systematic uncertainties are obtained using the same procedure as for the $\bar{F}_{2} 1$-parameter fit (see Section 7.1). The central values for $\bar{F}_{2}$ and $x \bar{F}_{3}$ for the 1-parameter and 2-parameter fits are very similar. Tables 7.2-7.5 give the values from the 2-parameter simultaneous $\bar{F}_{2}$ and $x \bar{F}_{3}$ fits, the combined (statistical and systematic) uncertainty and the correlation between the structure functions for each $x$ and $Q^{2}$ bin.

\subsection{COMPARISON WITH THEORY AND QCD}

Figures 7.3 and 7.4 show a comparison of $\mathrm{NuTeV}$ and CCFR data with two theory models. The plot in Figure 7.3 shows the ratio of $F_{2}\left(x, Q^{2}\right)$ to the TRVFS(MRST2001E) all $x$ bins. The $1 \sigma$ error band from the PDF set is also shown. The second theory curve shown on the plot is ACOTFFS(CTEQ5HQ1). Similarly, the plot in Figure 7.4 shows the same comparison for $x F_{3}\left(x, Q^{2}\right)$. All theory models have been corrected for target-mass effects using the Georgi-Politzer model [13]. These are important at high- $x$ and low- $Q^{2}$. For example, they increase the theory prediction by about $5 \%$ at $x=0.65$ and $Q^{2}=15 \mathrm{GeV}^{2}$. To make a direct comparison, the theory curves are also corrected for nuclear target effects. A standard treatment of nuclear effects, which are not well determined for neutrino scattering, is to apply corrections measured in charged-leptons scattering from nuclear targets. We use a multiplicative correction factor given by Equation 4.29 obtained from a fit to charged-lepton scattering on nuclear targets. The correction is independent of $Q^{2}$ and is small at intermediate $x$ but is large at low and high $x,(10 \%$ at $x=0.015$ and increases from $7 \%$ at $x=0.45$ to $15 \%$ at $x=0.65)$.

The data is in agreement with NLO QCD calculations for intermediate $x$. At low $x(x=$ 0.015 and $x=0.045)$ both data sets have different $Q^{2}$ shapes than theoretical predictions. At high- $x(x>0.5)$ our data are systematically above the theory predictions. Compared with the ACOTFFS(CTEQ5HQ1) model, our data has a similar $Q^{2}$ dependence, but in level is $10 \%$ higher at $x=0.65$, and $15 \%$ at $x=0.75$. In comparison with the TRVFS(MRST2001E) prediction at high- $x,(x=0.65$ and $x=0.75)$, our data has a different $Q^{2}$ dependence and 


\begin{tabular}{|c|c|c|c|c|c|c|}
\hline$x$ & $Q^{2}$ & $F_{2}$ & $F_{2}$ Uncertainty & $x F_{3}$ & $x F_{3}$ Uncertainty & Correlation \\
\hline 0.0150 & 1.2589 & 1.1534 & 0.0250 & 0.1843 & 0.0389 & -0.1148 \\
\hline 0.0150 & 1.9953 & 1.3332 & 0.0308 & 0.2183 & 0.0417 & -0.1582 \\
\hline 0.0150 & 3.1623 & 1.4724 & 0.0397 & 0.2679 & 0.0470 & -0.2252 \\
\hline 0.0150 & 5.0119 & 1.5661 & 0.0591 & 0.3226 & 0.0575 & -0.2393 \\
\hline 0.0150 & 7.9433 & 1.6393 & 0.1822 & 0.2756 & 0.1626 & -0.3664 \\
\hline 0.0450 & 1.2589 & 1.0901 & 0.0246 & 0.3803 & 0.0866 & -0.0426 \\
\hline 0.0450 & 1.9953 & 1.2283 & 0.0233 & 0.4098 & 0.0526 & 0.0079 \\
\hline 0.0450 & 3.1623 & 1.3476 & 0.0243 & 0.4640 & 0.0443 & -0.0010 \\
\hline 0.0450 & 5.0119 & 1.4714 & 0.0275 & 0.4746 & 0.0399 & 0.0096 \\
\hline 0.0450 & 7.9433 & 1.5303 & 0.0333 & 0.5000 & 0.0455 & -0.0934 \\
\hline 0.0450 & 12.5893 & 1.5785 & 0.0447 & 0.5374 & 0.0491 & -0.1220 \\
\hline 0.0450 & 19.9526 & 1.7131 & 0.1085 & 0.5692 & 0.1049 & -0.1893 \\
\hline 0.0800 & 1.2589 & 1.0646 & 0.0671 & 0.4008 & 0.4714 & -0.1067 \\
\hline 0.0800 & 1.9953 & 1.2169 & 0.0222 & 0.5394 & 0.0811 & 0.0003 \\
\hline 0.0800 & 3.1623 & 1.3032 & 0.0221 & 0.6070 & 0.0550 & 0.0525 \\
\hline 0.0800 & 5.0119 & 1.3665 & 0.0211 & 0.6611 & 0.0411 & 0.0835 \\
\hline 0.0800 & 7.9433 & 1.3885 & 0.0217 & 0.6372 & 0.0337 & 0.1116 \\
\hline 0.0800 & 12.5893 & 1.4391 & 0.0274 & 0.6596 & 0.0388 & 0.0468 \\
\hline 0.0800 & 19.9526 & 1.4412 & 0.0326 & 0.6788 & 0.0387 & 0.0158 \\
\hline 0.0800 & 31.6228 & 1.5139 & 0.0614 & 0.7280 & 0.0631 & -0.0002 \\
\hline
\end{tabular}

Table 7.2: $F_{2}$ and $x F_{3}$ values from simultaneous two parameter fit. Correlation between the structure functions are also provided. The quoted uncertainty includes both the statistical and systematic uncertainties. 


\begin{tabular}{|c|c|c|c|c|c|c|}
\hline$x$ & $Q^{2}$ & $F_{2}$ & $F_{2}$ Uncertainty & $x F_{3}$ & $x F_{3}$ Uncertainty & Correlation \\
\hline 0.1250 & 1.9953 & 1.1336 & 0.0631 & 0.7089 & 0.4465 & -0.0677 \\
\hline 0.1250 & 3.1623 & 1.2334 & 0.0198 & 0.6327 & 0.0740 & 0.0165 \\
\hline 0.1250 & 5.0119 & 1.2512 & 0.0193 & 0.6906 & 0.0492 & 0.1099 \\
\hline 0.1250 & 7.9433 & 1.2614 & 0.0171 & 0.7353 & 0.0332 & 0.1605 \\
\hline 0.1250 & 12.5893 & 1.2904 & 0.0196 & 0.7598 & 0.0309 & 0.2111 \\
\hline 0.1250 & 19.9526 & 1.2776 & 0.0233 & 0.7580 & 0.0335 & 0.1628 \\
\hline 0.1250 & 31.6228 & 1.2659 & 0.0267 & 0.7659 & 0.0322 & 0.1593 \\
\hline 0.1250 & 50.1187 & 1.3359 & 0.0553 & 0.7886 & 0.0586 & 0.1438 \\
\hline 0.1750 & 3.1623 & 1.1246 & 0.0366 & 0.5863 & 0.1922 & -0.0296 \\
\hline 0.1750 & 5.0119 & 1.1205 & 0.0193 & 0.7508 & 0.0703 & 0.0716 \\
\hline 0.1750 & 7.9433 & 1.1576 & 0.0164 & 0.7746 & 0.0376 & 0.1854 \\
\hline 0.1750 & 12.5893 & 1.1543 & 0.0175 & 0.7929 & 0.0339 & 0.2218 \\
\hline 0.1750 & 19.9526 & 1.1389 & 0.0183 & 0.7779 & 0.0280 & 0.2842 \\
\hline 0.1750 & 31.6228 & 1.1311 & 0.0225 & 0.7667 & 0.0315 & 0.2304 \\
\hline 0.1750 & 50.1187 & 1.0945 & 0.0266 & 0.7843 & 0.0308 & 0.3463 \\
\hline 0.1750 & 79.4328 & 1.1521 & 0.0830 & 0.8030 & 0.0861 & 0.3541 \\
\hline
\end{tabular}

Table 7.3: $F_{2}$ and $x F_{3}$ values from simultaneous two parameter fit. Correlation between the structure functions are also provided. The quoted uncertainty includes both the statistical and systematic uncertainties. 


\begin{tabular}{|c|c|c|c|c|c|c|}
\hline$x$ & $Q^{2}$ & $F_{2}$ & $F_{2}$ Uncertainty & $x F_{3}$ & $x F_{3}$ Uncertainty & Correlation \\
\hline 0.2250 & 5.0119 & 1.0330 & 0.0221 & 0.7713 & 0.1014 & 0.0535 \\
\hline 0.2250 & 7.9433 & 1.0257 & 0.0173 & 0.7582 & 0.0523 & 0.1415 \\
\hline 0.2250 & 12.5893 & 1.0002 & 0.0155 & 0.7483 & 0.0335 & 0.2449 \\
\hline 0.2250 & 19.9526 & 0.9956 & 0.0171 & 0.7646 & 0.0288 & 0.3485 \\
\hline 0.2250 & 31.6228 & 0.9545 & 0.0185 & 0.7380 & 0.0287 & 0.3163 \\
\hline 0.2250 & 50.1187 & 0.9246 & 0.0211 & 0.7118 & 0.0278 & 0.3358 \\
\hline 0.2250 & 79.4328 & 0.9121 & 0.0346 & 0.6938 & 0.0385 & 0.4089 \\
\hline 0.2750 & 5.0119 & 0.9046 & 0.0331 & 0.6478 & 0.1758 & 0.0266 \\
\hline 0.2750 & 7.9433 & 0.8831 & 0.0163 & 0.7323 & 0.0556 & 0.1529 \\
\hline 0.2750 & 12.5893 & 0.8751 & 0.0155 & 0.6952 & 0.0387 & 0.2369 \\
\hline 0.2750 & 19.9526 & 0.8424 & 0.0154 & 0.6615 & 0.0315 & 0.2618 \\
\hline 0.2750 & 31.6228 & 0.8244 & 0.0162 & 0.6654 & 0.0269 & 0.3419 \\
\hline 0.2750 & 50.1187 & 0.7680 & 0.0199 & 0.6425 & 0.0285 & 0.3719 \\
\hline 0.2750 & 79.4328 & 0.7539 & 0.0249 & 0.6400 & 0.0303 & 0.4588 \\
\hline 0.3500 & 7.9433 & 0.6850 & 0.0131 & 0.5490 & 0.0611 & 0.0825 \\
\hline 0.3500 & 12.5893 & 0.6721 & 0.0100 & 0.5713 & 0.0321 & 0.1804 \\
\hline 0.3500 & 19.9526 & 0.6320 & 0.0096 & 0.5561 & 0.0229 & 0.2740 \\
\hline 0.3500 & 31.6228 & 0.5922 & 0.0095 & 0.5332 & 0.0172 & 0.3948 \\
\hline 0.3500 & 50.1187 & 0.5630 & 0.0116 & 0.5214 & 0.0190 & 0.4014 \\
\hline 0.3500 & 79.4328 & 0.5415 & 0.0119 & 0.5037 & 0.0162 & 0.4878 \\
\hline 0.3500 & 125.8925 & 0.5202 & 0.0258 & 0.4785 & 0.0301 & 0.6140 \\
\hline
\end{tabular}

Table 7.4: $F_{2}$ and $x F_{3}$ values from simultaneous two parameter fit. Correlation between the structure functions are also provided. The quoted uncertainty includes both the statistical and systematic uncertainties. 


\begin{tabular}{|c|c|c|c|c|c|c|}
\hline$x$ & $Q^{2}$ & $F_{2}$ & $F_{2}$ Uncertainty & $x F_{3}$ & $x F_{3}$ Uncertainty & Correlation \\
\hline 0.4500 & 7.9433 & 0.4467 & 0.0168 & 0.3817 & 0.0897 & 0.0537 \\
\hline 0.4500 & 12.5893 & 0.4236 & 0.0087 & 0.3838 & 0.0348 & 0.1406 \\
\hline 0.4500 & 19.9526 & 0.3950 & 0.0079 & 0.3735 & 0.0230 & 0.2461 \\
\hline 0.4500 & 31.6228 & 0.3615 & 0.0075 & 0.3425 & 0.0170 & 0.3342 \\
\hline 0.4500 & 50.1187 & 0.3417 & 0.0086 & 0.3222 & 0.0167 & 0.3376 \\
\hline 0.4500 & 79.4328 & 0.3130 & 0.0086 & 0.2990 & 0.0143 & 0.3491 \\
\hline 0.4500 & 125.8925 & 0.3038 & 0.0136 & 0.2884 & 0.0180 & 0.4808 \\
\hline 0.5500 & 12.5893 & 0.2548 & 0.0077 & 0.2347 & 0.0383 & 0.0768 \\
\hline 0.5500 & 19.9526 & 0.2248 & 0.0057 & 0.2121 & 0.0210 & 0.1793 \\
\hline 0.5500 & 31.6228 & 0.2075 & 0.0055 & 0.2044 & 0.0154 & 0.2163 \\
\hline 0.5500 & 50.1187 & 0.1772 & 0.0061 & 0.1843 & 0.0128 & 0.3785 \\
\hline 0.5500 & 79.4328 & 0.1607 & 0.0061 & 0.1622 & 0.0125 & 0.2576 \\
\hline 0.5500 & 125.8925 & 0.1569 & 0.0104 & 0.1347 & 0.0159 & 0.2513 \\
\hline 0.6500 & 12.5893 & 0.1362 & 0.0067 & 0.1330 & 0.0340 & 0.0966 \\
\hline 0.6500 & 19.9526 & 0.1150 & 0.0042 & 0.1206 & 0.0177 & 0.1804 \\
\hline 0.6500 & 31.6228 & 0.0985 & 0.0035 & 0.1126 & 0.0117 & 0.2456 \\
\hline 0.6500 & 50.1187 & 0.0876 & 0.0039 & 0.0832 & 0.0103 & 0.2288 \\
\hline 0.6500 & 79.4328 & 0.0786 & 0.0043 & 0.0719 & 0.0107 & 0.1101 \\
\hline 0.6500 & 125.8925 & 0.0707 & 0.0062 & 0.0696 & 0.0117 & 0.2309 \\
\hline 0.7500 & 12.5893 & 0.0626 & 0.0051 & 0.0499 & 0.0293 & 0.0305 \\
\hline 0.7500 & 19.9526 & 0.0495 & 0.0026 & 0.0479 & 0.0134 & 0.1028 \\
\hline 0.7500 & 31.6228 & 0.0382 & 0.0020 & 0.0352 & 0.0075 & 0.0904 \\
\hline 0.7500 & 50.1187 & 0.0333 & 0.0020 & 0.0343 & 0.0077 & 0.0541 \\
\hline 0.7500 & 79.4328 & 0.0308 & 0.0030 & 0.0254 & 0.0100 & -0.1176 \\
\hline
\end{tabular}

Table 7.5: $F_{2}$ and $x F_{3}$ values from simultaneous two parameter fit. Correlation between the structure functions are also provided. The quoted uncertainty includes both the statistical and systematic uncertainties. 
is $15-20 \%$ higher in level at $x=0.65$, and $25-40 \%$ at $x=0.75$.

A different theoretical treatment of nuclear effects could make a sizable difference at small and large $x$. We assume the same correction as in charged lepton data. Our result perhaps indicates that neutrino scattering favors smaller nuclear effects at high- $x$ than are found in charged-lepton scattering. At small $x$, new theoretical calculations show that in the shadowing region the nuclear correction has $Q^{2}$ dependence $[42,43]$. The standard nuclear correction obtained from a fit to charged lepton data implies a suppression of $10 \%$ independent of $Q^{2}$ at $x=0.015$, while for $x=0.015$, reference [43] finds a suppression of $15 \%$ at $Q^{2}=1.25 \mathrm{GeV}^{2}$ and a suppression of $3.4 \%$ at $Q^{2}=7.94 \mathrm{GeV}^{2}$. Figure 7.5 shows the comparison with theory for the two lowest $\mathrm{x}$ bins $(x=0.015$ and $x=0.045)$. If the TRVFS(MRST2001E) prediction is calculated using the nuclear correction from reference [43] at low $x(\leq 0.045)$ instead of the standard nuclear correction agreement with data improves. Theory models shown here have, in part, a correlation with CCFR data, which is used in the global PDF fits. NuTeV data will be included in the next generation PDF fits.

\subsection{LOGARITHMIC SLOPES}

The logarithmic slope of the non-singlet quark evolution depends on $\alpha_{s}\left(Q^{2}\right)$

$$
\frac{d \ln q^{N S}}{d \ln Q^{2}} \propto \alpha_{s}\left(Q^{2}\right)
$$

However, over the limited kinematic range of our data it can be approximated very well with constant $C$

$$
\frac{d \ln q^{N S}}{d \ln Q^{2}}=C
$$

We can obtain the logarithmic slopes from the measured $x F_{3}$, which gives the non-singlet quark distribution, for each $\mathrm{x}$ bin. Figure 7.6 shows the logarithmic slope compared with two NLO theoretical predictions TRVFS(MRST2001E) and ACOTFFS(CTEQ5HQ1). The logarithmic slope for the non-singlet structure function $x F_{3}$ depends on $Q^{2}$. Therefore, for the theoretical predictions we take the average logarithmic slope in the $Q^{2}$ range of our 


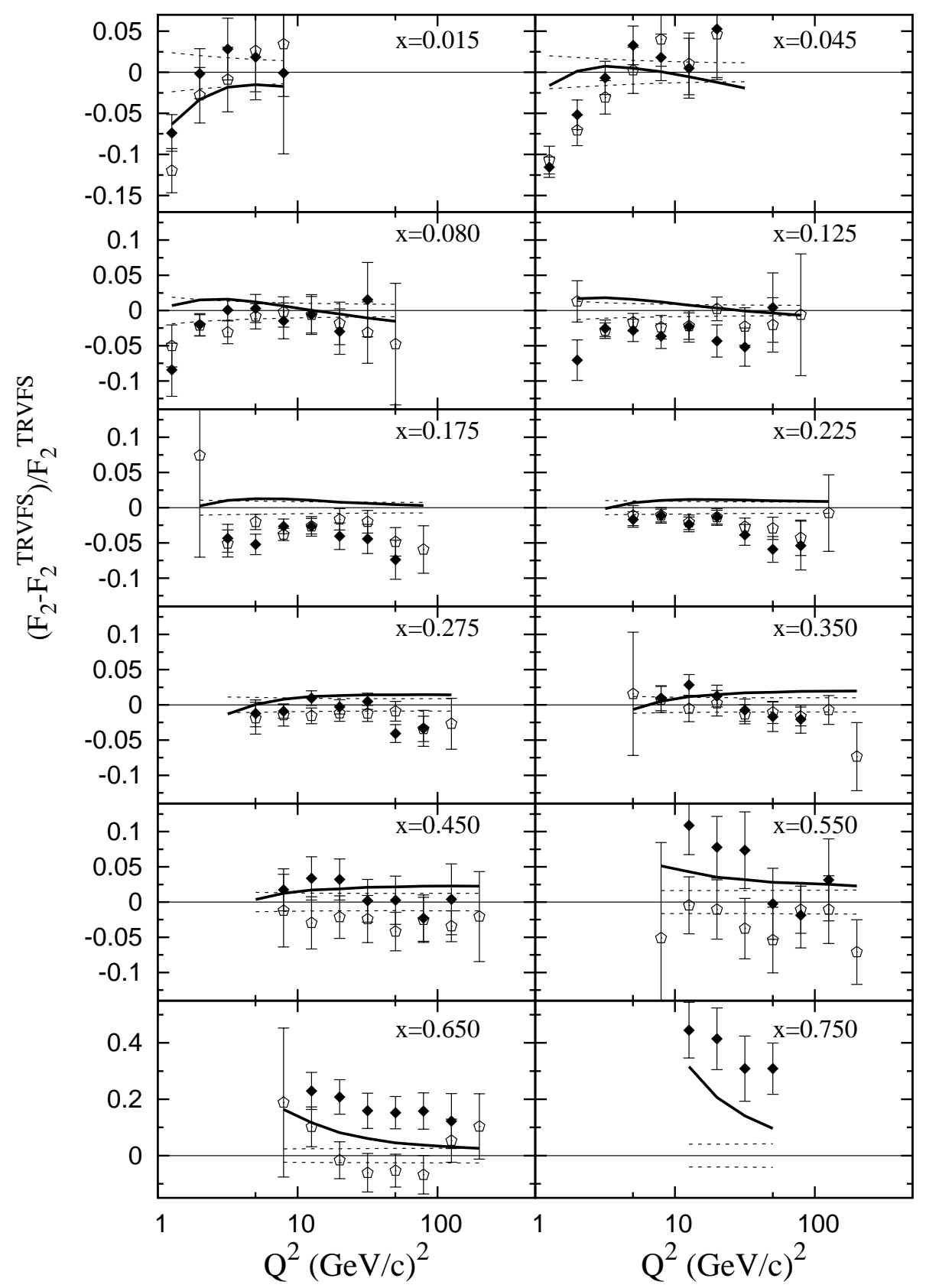

Figure 7.3: $\quad F_{2}\left(x, Q^{2}\right)$ fractional difference $\frac{F_{2}-F_{2}^{T R V F S}}{F_{2}^{T R V F S}}$ with respect to the TRVFS(MRST2001E) model. Data points are NuTeV (solid dots) and CCFR (open circles). Theory curves are ACOTFFS(CTEQ5HQ1) (solid line) and TRVFS(MRST2001E) $\pm 1 \sigma$ (dashed lines). Theory curves are corrected for target mass and nuclear effects. 


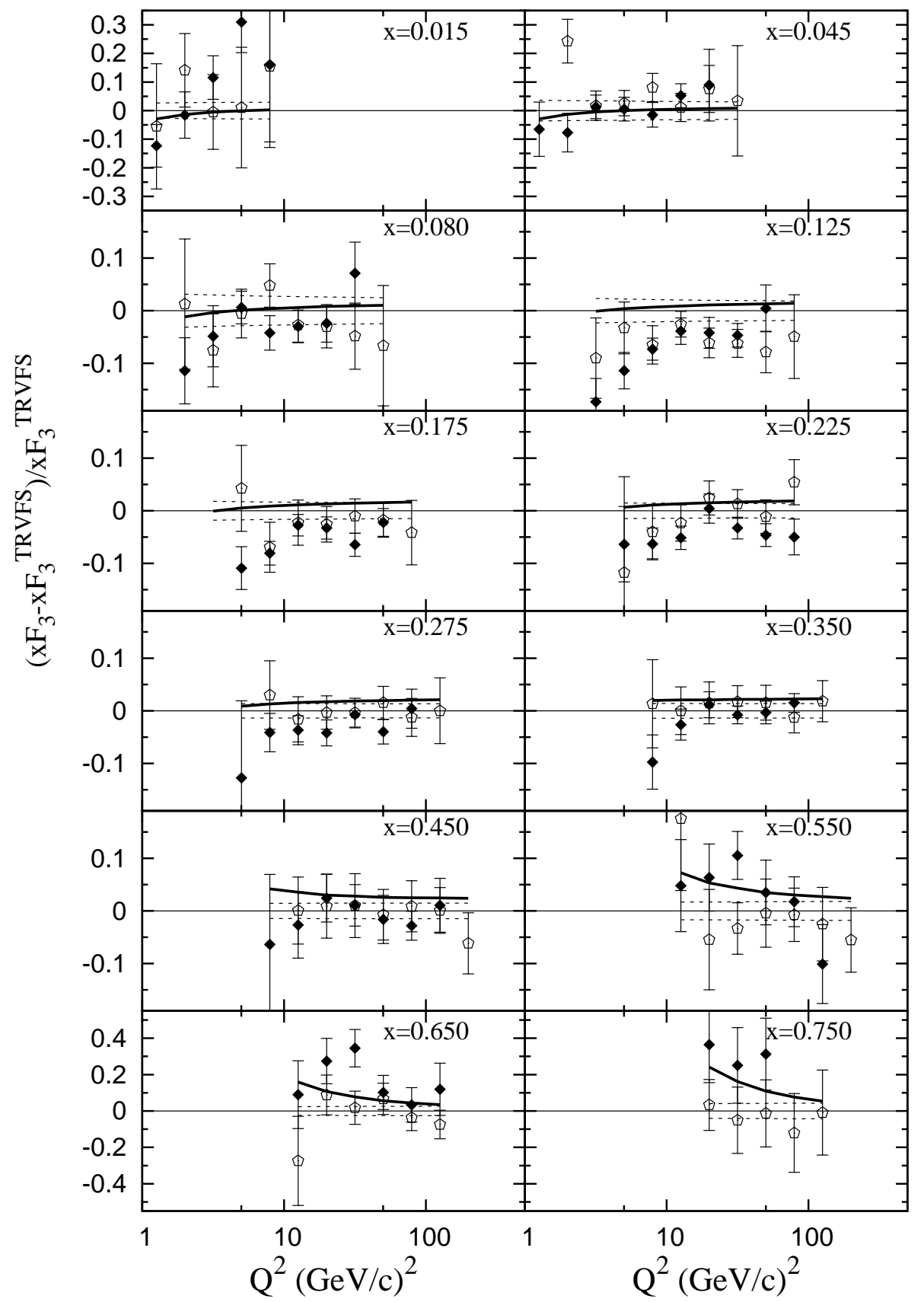

Figure 7.4: $\quad x F_{3}\left(x, Q^{2}\right)$ fractional difference $\frac{x F_{3}-x F_{3}^{T R V F S}}{x F_{3}^{T R V F S}}$ with respect to the TRVFS(MRST2001E) model. Data points are NuTeV (solid dots) and CCFR (open circles). Theory curves are ACOTFFS(CTEQ5HQ1) (solid line) and TRVFS(MRST2001E) $\pm 1 \sigma$ (dashed lines). Theory curves are corrected for target mass and nuclear effects. 

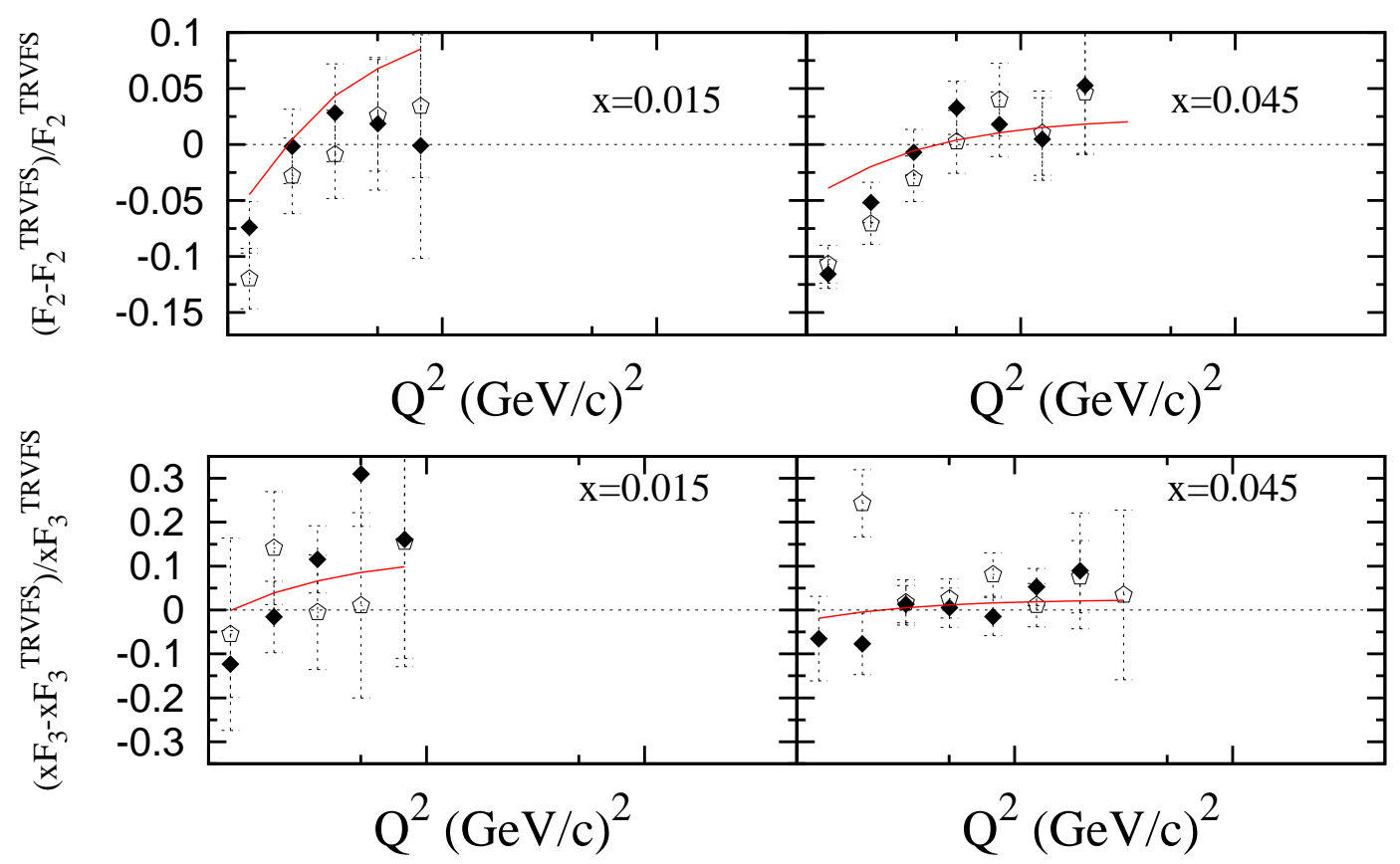

Figure 7.5: $F_{2}\left(x, Q^{2}\right)$ (top) and $x F_{3}$ (bottom) fractional difference with respect to the TRVFS(MRST2001E) model. Data points are NuTeV (solid dots) and CCFR (open circles). Red curves represents the TRVFS(MRST2001E) with the nuclear correction from reference [43] instead of the standard nuclear correction from charged lepton data. Theory curves are corrected for target mass effects. 


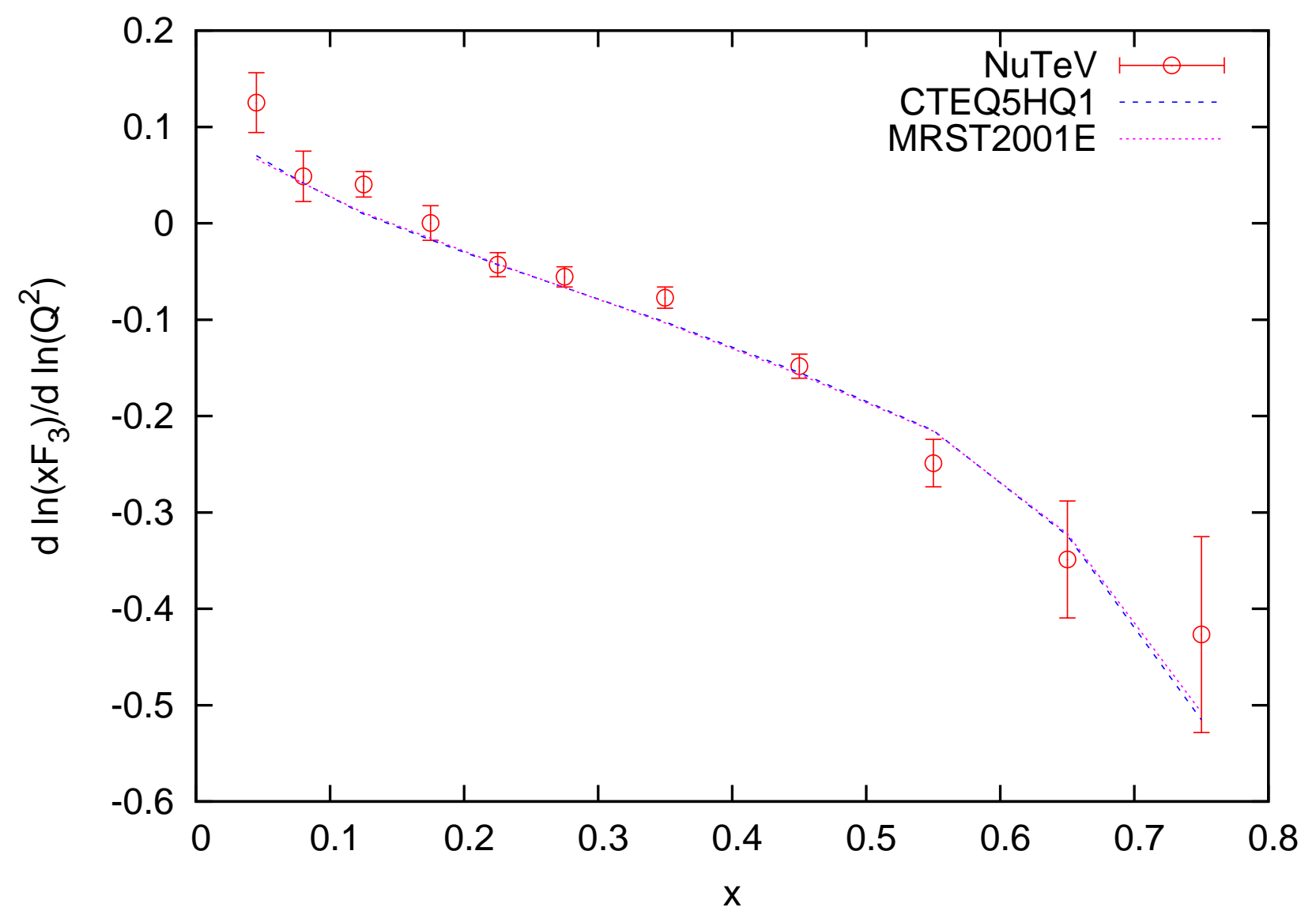

Figure 7.6: Comparison between the QCD logslopes for NuTeV and two NLO QCD theory predictions TRVFS(MRST2001E) and ACOTFFS(CTEQ5HQ1). 


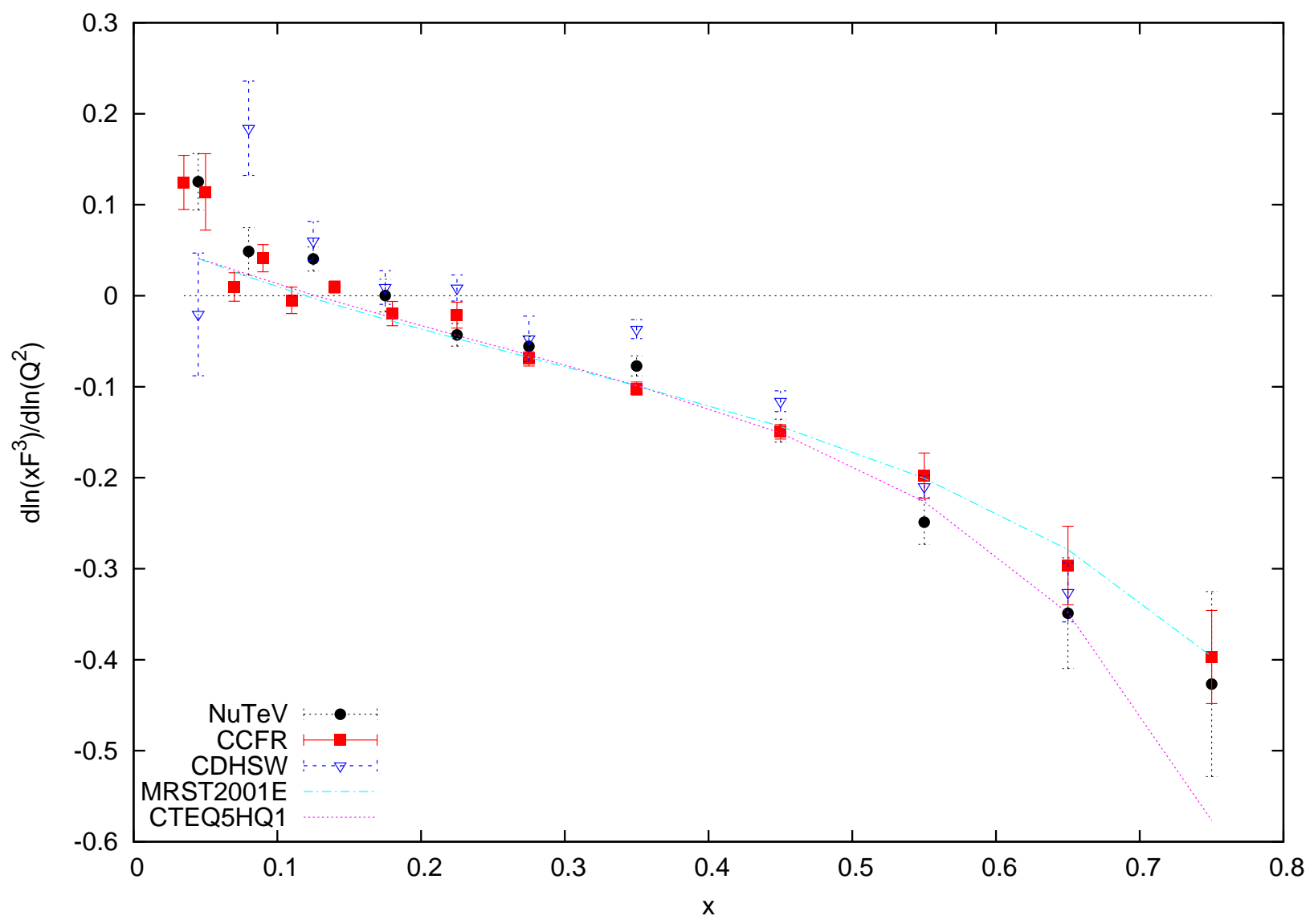

Figure 7.7: Comparison between the QCD logslopes for NuTeV, CCFR, CDHSW and two NLO QCD theory predictions TRVFS(MRST2001E) and ACOTFFS(CTEQ5HQ1). 
data. Both theoretical models are corrected for target mass and nuclear effects. In order to minimize the effect of non-perturbative QCD effects we use data above $Q^{2}>5 G e V^{2} / c^{2}$. Our result is in reasonable agreement with NLO QCD predictions. At low x a $Q^{2}$ dependence of the nuclear correction (discussed in the previous section) will change the logarithmic slopes.

Figure 7.7 shows a comparison of the logarithmic slopes obtained from the $x F_{3}$ structure function measured by $\mathrm{NuTeV}, \mathrm{CCFR}$ and CDHSW. For each experiment the fit was performed in the $Q^{2}$ range specific to the experiment with the requirement $Q^{2}>5 G e V^{2} / c^{2}$. The two theoretical predictions TRVFS(MRST2001E) and ACOTFFS(CTEQ5HQ1) are also shown in the plot. For this plot the logarithmic slope for the theoretical predictions are the average logarithmic slope for the $Q^{2}$ range $5<Q^{2}<200 G e V^{2} / c^{2}$. The difference in the $Q^{2}$ ranges for different experiments explains partially the observed difference in the logarithmic slopes between them.

These logarithmic slopes provide a visual comparison between structure functions and theory of QCD. NuTeV result agrees with QCD theory. 


\subsection{CONCLUSIONS}

This thesis presents a new measurement of the differential cross section for neutrino and antineutrino deep inelastic scattering off an iron target. This measurement is the most precise measurement to date, which was achieved by having better control over the systematic uncertainties compared with previous measurements. For the first time, a complete pointto-point covariance matrix is obtained to account for the correlations between data points.

This measurement is in agreement with the previous measurements of the differential cross section at intermidiate $\mathrm{x}(x<0.4)$ for neutrino DIS. At high $x$ this measurement is systematically above the CCFR measurement for both neutrino and anti-neutrino cross sections. The discrepancy is $4 \pm 1 \%$ at $x=0.45,9 \pm 2 \%$ at $x=0.55$, and increases with $x$ up to $18 \pm 2 \%$ at $x=0.65$. Experimental improvements implemented by $\mathrm{NuTeV}$ for this measurement account for a large fraction of the difference seen. After accounting for all known experimental differences both experiments are in $1.2 \sigma$ agreement.

This thesis presents a measurement of the ratio of the total anti-neutrino to neutrino iron cross section. The value for an isoscalar iron target is $\sigma^{\bar{\nu}} / \sigma^{\nu}=0.505 \pm 0.0018($ stat $) \pm$ 0.0029 (syst) for the energy range between 30 and $360 \mathrm{GeV}$. This value gives the most precise measurement of this quantity to date.

The structure functions $F_{2}$ and $x F_{3}$ have been determined from the $y$-dependence of the differential cross sections. These structure functions are important inputs for determining the parton distributions and to better constrain global PDF fits. The measurement agrees with neutrino iron structure functions for $x<0.4$. As is the case for the differential cross sections, the result gives a higher value for $F_{2}$ and $x F_{3}$ at high $x$ than previous results. The source of a large fraction of this difference is understood and due to improvements in NuTeV's measurement. $F_{2}$ and $x F_{3}$ are in good agreement with NLO QCD predictions 
in the intermediate $\mathrm{x}$ range $(0.1<x<0.5)$. For $x<0.1$ the $Q^{2}$ shape differs from the shape of the theoretical predictions. This may indicate that the nuclear correction for neutrino scattering is $Q^{2}$ dependent. New theoretical calculations of the nuclear correction for neutrino iron scattering in this region predict a $Q^{2}$ dependence. At $x>0.5$ the measurement is systematically above NLO QCD predictions, which indicates that perhaps the nuclear correction at high $x$ is different for neutrino scattering compared to that for charge lepton scattering.

In the future a high-y differential cross section from the sample with target analyzed muon will be added to this cross section sample. This will give us additional cross section points at low $x$ and high $y$ which are needed to constrain better the two parameter fits for $F_{2}$ and $R_{L}$ structure functions. The strong coupling constant $\alpha_{S}$ will be measured from the evolution of the non-singlet structure function, $x F_{3}\left(x, Q^{2}\right)$, and from the combined evolution of the singlet and non-singlet structure functions, $F_{2}\left(x, Q^{2}\right)$ and $x F_{3}\left(x, Q^{2}\right)$, at high $x$.

The neutrino cross section is well measured at high energy. However, at low energy the neutrino cross section is poorly known. The MINOS collaboration will use a high intensity low energy beam to measure the neutrino cross section, the knowledge of which is of great importance for the precise measurement of neutrino oscillations. MINERvA collaboration will use the same beam but different detector to measure neutrino cross section at low energy. Their detector is designed to measure the cross section for different nuclear targets. This will allow to obtain the nuclear effects for neutrino scattering. Then the measured nuclear correction for neutrino scattering will be used to compare the existing neutrino DIS data with charge lepton DIS data. 


\section{BIBLIOGRAPHY}

[1] P. Berge et al. (CDHSW Collaboration), Z. Phys. C49, 607 (1991).

[2] U. K. Yang, Ph. D. Thesis, University of Rochester, (2001), UR-1583.

[3] J. Conrad, M. H. Shaevitz, and T. Bolton, Rev. Mod. Phys. 704 (1998).

[4] G. Sterman et. al, Rev. Mod. Phys. 67157 (1995).

[5] J. D. Bjorken, Phys. Rev. 179, 1547 (1969).

[6] R. P. Feynman, Phys. Rev. Lett. 23, 1415 (1969).

[7] C. G. Callan and D. Gross, Phys. Rev. Lett. 22, 156 (1969).

[8] E. D. Bloom et al. (MIT-SLAC Collaboration), Phys. Rev. Lett. 23, 930 (1969.).

[9] F. J. Yndurain, The Theory of Quark and Gluon Interaction. Berlin: Springer-Verlag, 1993.

[10] D. E. Soper, J. C. Collins and G. Stermann, Factorization of Hard Processes in QCD, Singapore: World Scientific, 1989

[11] Yu. Dokshitzer, Soviet Phys. JETP 46, 641 (1977);

V. N. Gribov and L. N. Lipatov, Soviet J. Nucl. Phys. 15, 438,675, (1972);

L. N. Lipatov, Soviet J. Nucl. Phys. 20, 95, (1975);

G. Altarelli and G. Parisi, Nucl. Phys. B126, 298 (1977).

[12] G. Altarelli and G. Martinelli, Phys. Lett. 76B, 89 (1978).

[13] H. Georgi and H. D. Politzer, Phys. Rev. D14, 1829 (1976).

[14] R. H. Bernstein et. al., FERMILAB-TM-1884 (1994),

J. Yu et. al., FERMILAB-TM-2040 (1998).

[15] D. A. Harris et. al, Nucl. Instr. Meth. A447, 377 (2000).

[16] T. A. Gabriel and D. E. Groom, Nucl. Instr. Meth. A338, 336 (1994). 
[17] Finite element analysis software, http://www.ansys.com.

[18] A. J. Buras and K. J. F. Gaemers, Nucl. Phys. B132, 1978 (249).

[19] M. Kobayashi and T. Maskawa, Prog. Theor. Phys. 49, 652, (1973)

[20] S. A. Rabinowitz et al. (CCFR Collaboration), Phys. Rev. Lett. 70, 1993 (134).

[21] M. Arneodo et al. (NMC Collaboration), Nucl. Phys. B 487, 3 (1997).

[22] A. Hawker et. al, Phys. Rev. Lett. 80, 3715 (1998).

[23] M. Gluck et al. (GRV Collaboration), Z. Phys. C67, 433 (1995).

[24] A. Bodek and U. K. Yang, Nucl. Phys. Proc. Suppl. 11270 (2002).

[25] L. W. Whitlow et. al, Phys. Lett. B282 475 (1992).

[26] A. C. Benvenuti et. al, Phys. Lett. B223 485 (1989); A. C. Benvenuti et. al, Phys. Lett. B236 592 (1989).

[27] M. Arneodo et. al, Nucl. Phys. B483 3 (1997).

[28] L. W. Whitlow et. al, Phys. Lett. B250 193 (1990).

[29] R. M. Barnet, Phys. Rev. D14, 70 (1976).

[30] P. Vilain et. al, Eur. Phys. J. C11 19 (1999);

P. Astier et. al, Phys. Lett. B486 35(2000).

[31] D. Yu. Bardin and V. A. Dokuchaeva, JINR-E2-86-260 (1986).

[32] W. Seligman, Ph. D. Thesis, Columbia University, Nevis 292 (1997).

[33] Arie Bodek, Inkyu Park, Un-ki Yang, Nucl. Phys. Proc. Suppl. 139 (2005) 113-118. [arXiv:hep-ph/0411202].

[34] R. Thorne and R. Roberts, Phys. Lett. B421 (1998) 303. A. D. Martin et. al. Eur. Phys. J. C18 117 (2000).

[35] A. D. Martin, R. G. Roberts, W. James Stirling, R. S. Thorne, Eur.Phys.J.C14 133 (2000).

[36] CERN computer program POISSON; see R. Holsinger, CERN Computer Center Program Library T604.

[37] B. King et al. (CCFR Collaboration), Nucl. Instr. Meth. A302, 254 (1991).

[38] A. D. Martin, R. G. Roberts, W. J. Stirling, R. S. Thorne, Eur. Phys. J. C28 (2003) $455-473$. 
[39] M. A. G. Aivazis, J. C. Collins, F. I. Olness, and W. K. Tung, Phys. Rev. D50, 3102 (1994).

[40] H. L. Lai et. al., Eur. Phys. J. C12 (2000) 375-392.

[41] H. Georgi and H. D. Politzer, Phys. Rev. D14 (1976) 1829.

[42] S.A. Kulagin, R. Petti, [arXiv:hep-ph/0412425].

[43] J. W. Qiu and I. Vitev, Phys. Lett. B587, (2004) 52 [arXiv:hep-ph/0401062].

[44] M. Tzanov et al., PRECISE MEASUREMENT OF NEUTRINO AND ANTI- NEUTRINO DIFFERENTIAL CROSS SECTIONS. FERMILAB-PUB-05-453-E (Sep 2005) 27p. [HEP-EX 0509010] 


\title{
APPENDIX A
}

\section{THE NUTEV COLLABORATION}

\author{
R. A. Johnson, N. Suwonjandee, M. Vakili
}

University of Cincinnati

J. Conrad, B. T. Fleming, J. Formaggio, J. H. Kim, S. Koutsoliotas, C. McNulty,

A. Romosan, M. H. Shaevitz, P. Spentzouris, E. G. Stern, A. Vaitaitis, E. D. Zimmerman Columbia University

R. H. Bernstein, L. Bugel, M. J. Lamm, W. Marsh, P. Nienaber, N. Tobien, J. Yu. Fermi National Accelerator Laboratory

T. Adams, A. Alton, T. Bolton, J. Goldman, M. Goncharov

Kansas State University

L. de Barbaro, D. Buchholz, H. Schellman, G. P. Zeller

Northwestern University

J. Brau, R. B. Drucker, R. Frey, D. Mason

University of Oregon

S. Avvakumov, P. de Barbaro, A. Bodek, H. Budd

D. A. Harris, K. S. McFarland, W. K. Sakumoto,U. K. Yang

University of Rochester

S. Boyd, J. McDonald, D. Naples, V. Radescu, M. Tzanov

University of Pittsburgh 


\section{APPENDIX B}

\section{PUBLICATION}

The remainder of this dissertation contains the article presenting this result that was submitted for publication. The paper was submitted for publication to Physical Review D in September 2005 waiting for approval [44]. 


\section{Precise Measurement of Neutrino and Anti-neutrino Differential Cross Sections}

M. Tzanov, D. Naples, S. Boyd, J. McDonald, V. Radescu

Department of Physics, University of Pittsburgh, PA 15260

R. A. Johnson, N. Suwonjandee, M. Vakili

University of Cincinnati, Cincinnati, OH 45221

J. Conrad, B. T. Fleming, J. Formaggio, J. H. Kim, S. Koutsoliotas,

C. McNulty, A. Romosan, M. H. Shaevitz, P. Spentzouris,

E. G. Stern, A. Vaitaitis, E. D. Zimmerman

Columbia University, New York, NY 10027

R. H. Bernstein, L. Bugel, M. J. Lamm, W. Marsh, P. Nienaber, N. Tobien, J. Yu Fermi National Accelerator Laboratory, Batavia, IL 60510

T. Adams, A. Alton, T. Bolton, J. Goldman, M. Goncharov Kansas State University, Manhattan, KS 66506

L. de Barbaro, D. Buchholz, H. Schellman, G. P. Zeller Northwestern University, Evanston, IL 60208

J. Brau, R. B. Drucker, R. Frey, D. Mason University of Oregon, Eugene, OR 97403

S. Avvakumov, P. de Barbaro, A. Bodek, H. Budd, D. A. Harris, K. S. McFarland, W. K. Sakumoto,U. K. Yang University of Rochester, Rochester, NY. 14627

The NuTeV experiment at Fermilab has obtained a unique high statistics sample of neutrino and anti-neutrino interactions using its high-energy sign-selected beam. We present a measurement of the differential cross section for charged-current neutrino and anti-neutrino scattering from iron. Structure functions, $F_{2}\left(x, Q^{2}\right)$ and $x F_{3}\left(x, Q^{2}\right)$, are determined by fitting the inelasticity, $y$, dependence of the cross sections. This measurement has significantly improved systematic precision as a consequence of more precise understanding of hadron and muon energy scales.

PACS numbers: 12.38.Qk, 13.15.+g, 13.60.Hb 


\section{INTRODUCTION}

Deep inelastic scattering (DIS), the scattering of a high energy lepton off a quark inside a nucleon, has been a proving ground for QCD, the theory of strong interactions. Charged-leptons and neutrinos have been used to measure parton densities and their QCD evolution with high-precision over a wide range in $Q^{2}$. Uniquely, neutrino DIS, via the weak interaction probe, allows simultaneous measurement of two structure functions: $F_{2}\left(x, Q^{2}\right)$ and the parity-violating structure function, $x F_{3}\left(x, Q^{2}\right)$.

In this paper we present a new measurement of highenergy neutrino and anti-neutrino differential cross sections from high-statistics data samples. The differential dependence of neutrino and anti-neutrino cross sections on Bjorken scaling variable, $x$, and inelasticity, $y$, provide the most model-independent physics observable for this process [1]. Previous high-statistics measurements of the neutrino and anti-neutrino differential cross section have been reported [2], [3]. This experiment has two improvements: first, a sign-selected beam allowed separate neutrino and antineutrino running and, second, a calibration beam continuously measured the detector's response. The largest experimental uncertainties in previous measurements arose from knowledge of energy scale and detector response functions [4], [5]. $\mathrm{NuTeV}$ addressed this by using a dedicated calibration beam of hadrons, electrons and muons that alternated with neutrino running once every minute throughout the one year data-taking peroid. The calibration beam was used to measure the detector response for hadrons and muons over a wide range of energies (5-200 GeV). Detector response functions and energy scales for muons and hadrons were mapped over the full active area of the detector. Energy scales for muons and hadrons were determined to a precision of $0.43 \%$ for hadrons and $0.7 \%$ for muons [6]. NuTeV's other improvement was separate neutrino and antineutrino running. NuTeV ran in two modes, $(\nu$ and $\bar{\nu})$, with the muon spectrometer polarity always set to focus the primary charged-lepton from the interaction vertex (e.g. $\mu^{-}$in $\nu$-mode or $\mu^{+}$in $\bar{\nu}$-mode). In determining the charged-current differential cross sections, this allowed better and more uniform acceptance in the two running modes and removed ambiguity in the muon sign determination present in a mixed $\nu$ and $\bar{\nu}$ beam.

The rest of this paper is organized into four parts; Section II describes the NuTeV detector, Section III gives the cross section extraction method and results, and Section

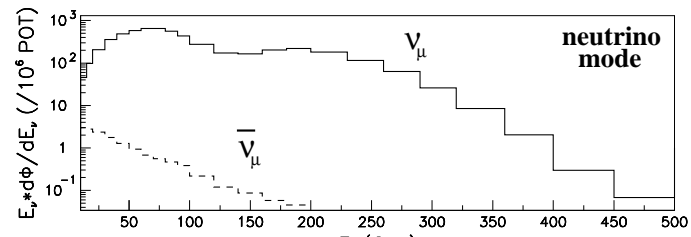

$\mathrm{E}_{\nu}(\mathrm{GeV})$

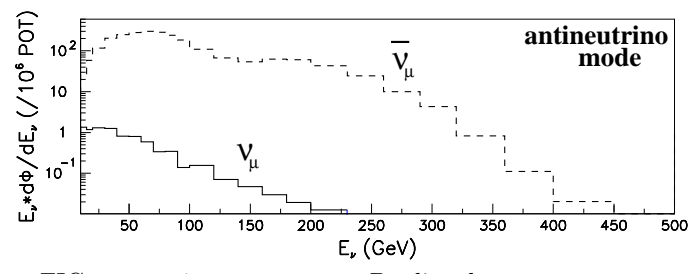

FIG. $1 . \quad$ Predicted energy

spectrum of interacted muon-neutrinos anti-neutrinos when the SSQT is set to select neutrinos (top) and antineutrinos (bottom).

IV presents the extracted structure functions and the results are discussed in Section V.

\section{NUTEV EXPERIMENT}

The NuTeV experiment collected data during 1996-97 using separate high-purity $\nu_{\mu}$ and $\bar{\nu}_{\mu}$ beam produced by the Sign-Selected Quadrupole Train (SSQT) beamline. A dipole magnet after the one-interaction-length beryllium oxide production target allowed the sign of secondary particles to be selected. Neutrinos (or anti-neutrinos) are produced when sign-selected secondary pions and kaons decay in the $440 \mathrm{~m}$ decay region located just downstream of SSQT optics. The NuTeV neutrino detector is $1.4 \mathrm{~km}$ downstream of the beryllium oxide production target. Neutrino energies ranged from 30-500 GeV. Figure 1 shows a prediction of the interacting $\nu_{\mu}$ and $\bar{\nu}_{\mu}$ flux in each mode with it's small contribution from the wrong-sign background. The interacted neutrino fraction from $\bar{\nu}(\nu)$ in $\nu(\bar{\nu})$-mode is $3 \times 10^{-4}\left(4 \times 10^{-3}\right)$.

The $\mathrm{NuTeV} / \mathrm{CCFR}$ detector consisted of an $18 \mathrm{~m}$ long, 690 ton target calorimeter with a mean density of $4.2 \mathrm{~g} / \mathrm{cm}^{3}$, followed by a 420 ton iron toroidal spectrometer. The target was composed of 168 steel plates, each $3 \mathrm{~m} \times 3 \mathrm{~m} \times 5.15 \mathrm{~cm}$, instrumented with liquid scintillator counters placed every two steel plates and drift chambers 


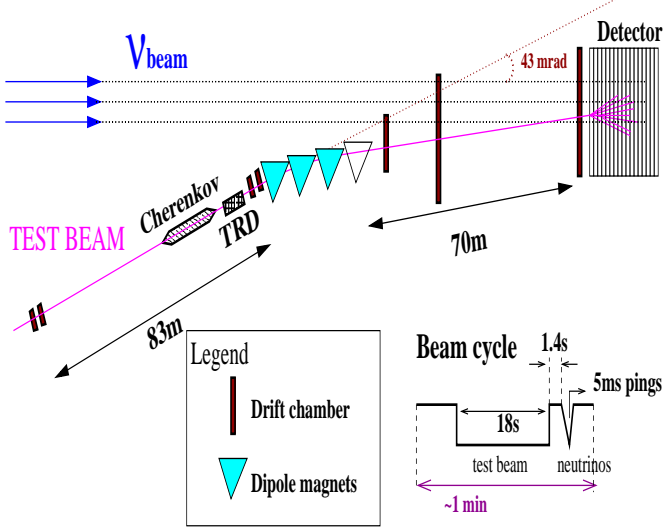

FIG. 2. Sketch of calibration beam spectrometer configuration. The calibration beam ran alternating with neutrinos once a minute for the entire $\mathrm{NuTeV}$ data-taking period.

spaced every four plates. NuTeV refurbished the CCFR detector by replacing the scintillator oil and reconditioning the drift chambers.

Immediately downstream of the target-calorimeter was a magnetized iron toroidal spectrometer with inner radius $12.5 \mathrm{~cm}$ and outer radius $175 \mathrm{~cm}$ used to measure the momentum of high-energy muons exiting the downstream end of the target. The toroid spectrometer consisted of three magnetized sections each followed by a drift chamber station. Two additional drift chamber stations were located a few meters downstream of the last magnetic section to analyze the highest momentum muons. The magnetic field in each toroidal section $(\sim 15 \mathrm{kG})$ was produced by four copper coils which emerged through the center hole. The magnetic field is azimuthal everywhere except for a small radial component in the region of the supporting feet and air gap between top and bottom halves of the washers. The azimuthal component of the field in the first toroid had an additional small asymmetry (with respect to vertical) due to a shorted coil on the west side. The detailed geometry of the spectrometer, (including the missing coil), was input to ANSYS simulation [7] to compute the magnetic field map.

A dedicated in situ calibration beam was used to determine the energy response of the calorimeter and spectrometer to hadrons, muons, and electrons and to map the response over the face of the detector. The tolerance of the calibration spectrometer to measure a beam particle's absolute momentum was $0.3 \%$. The configuration is shown in Figure 2. Details of the calibration and response measurements are given in [6]. The hadronic resolution of the calorimeter was determined to be $\frac{\sigma}{E}=\frac{86 \%}{\sqrt{E}} \oplus 2.2 \%$ with an absolute scale uncertainty of $\frac{\delta E}{E}=0.43 \%$. The latter uncertainty is dominated by the statistical precision in calibrating the time dependence of the counter response over a transverse grid for each counter.

The absolute scale of the toroid spectrometer was calibrated with muons that were steered over the active area of the spectrometer. The magnetic field map from the ANSYS simulation was checked with the $50 \mathrm{GeV}$ muon calibration beam. The mean reconstructed muon momentum compared with the muon calibration beam had a $1 \sigma$ variation of $0.63 \%$ over the active area of the toroid. This field map uncertainty is attributed to variation in magnetic susceptibility and thickness of the steel. The field map determination dominates the absolute muon energy scale uncertainty of $0.7 \%$.

\section{CROSS SECTION MEASUREMENT}

In charged-current (CC) neutrino DIS the $\nu$ scatters off a quark in the nucleon via exchange of a virtual $W$-boson. The cross section can be expressed in terms of structure functions, $2 x F_{1}\left(x, Q^{2}\right), F_{2}\left(x, Q^{2}\right)$, and $x F_{3}\left(x, Q^{2}\right)$

$$
\begin{aligned}
\frac{d^{2} \sigma^{\nu(\bar{\nu})}}{d x d y}= & \frac{G_{F}^{2} M E}{\pi}\left(\left[1-y\left(1+\frac{M x}{2 E}\right)+\frac{y^{2}}{2} \frac{1+\left(\frac{2 M x}{Q}\right)^{2}}{1+R_{L}}\right]\right. \\
& \left.F_{2}\left(x, Q^{2}\right) \pm\left[y-\frac{y^{2}}{2}\right] x F_{3}\left(x, Q^{2}\right)\right)
\end{aligned}
$$

where $G_{F}$ is the Fermi weak coupling constant, $M$ is the proton mass, $E_{\nu}$ is the incident neutrino energy in the lab frame, and $y$, the inelasticity, is the fraction of energy transferred to the hadronic system. The $x F_{3}$ term is added for neutrino interactions and is subtracted for antineutrinos. $R_{L}\left(x, Q^{2}\right)$, the ratio of the cross section for scattering from longitudinally to transversely polarized W-bosons relates $F_{2}\left(x, Q^{2}\right)$ and $2 x F_{1}\left(x, Q^{2}\right)$

$$
2 x F_{1}=F_{2}\left(\frac{1+\left(\frac{2 M x}{Q}\right)^{2}}{1+R_{L}\left(x, Q^{2}\right)}\right) .
$$

Structure functions depend on $x$, the Bjorken scaling variable, and $Q^{2}$, the four momentum squared of the virtual W-boson. 
Relativistic invariant kinematic variables, $x, y$, and $Q^{2}$ can be evaluated in the lab frame using the three experimentally measured quantities: $E_{\mu}$, energy of the outgoing primary charged-lepton, $E_{\mathrm{HAD}}$ the energy deposited at the hadronic vertex, and $\theta_{\mu}$, the scattering angle of the primary muon.

$$
\begin{gathered}
x=\frac{4 E_{\nu} E_{\mu} \sin ^{2} \frac{\theta_{\mu}}{2}}{2 M E_{\mathrm{HAD}}}, \\
y=E_{\mathrm{HAD}} / E_{\nu}, \text { and } \\
Q^{2}=2 M x y E_{\nu}
\end{gathered}
$$

where the neutrino energy, $E_{\nu}=E_{\mu}+E_{\mathrm{HAD}}$, is reconstructed from the measured final state particle energies.

\section{A. Event Reconstruction}

Events used in this analysis were triggered by the presence of a penetrating muon track determined by in-time hits in scintillation counters in the most downstream region of the target calorimeter and in the first station of the toroid spectrometer. This allowed acceptance for charged current events down to zero hadron energy.

$E_{\mathrm{HAD}}$ is determined by summing the pulse heights of consecutive counters from just downstream of the event vertex to five counters beyond the end of the shower region. Longitudinal position of the event vertex is defined as the first of at least two consecutive counters with greater than four times the energy deposited by a minimum ionizing particle (MIP). ${ }^{1}$ The end of the shower region is the last counter before three consecutive counters with less than four MIP's. Energy deposited by the primary muon in this region is removed by subtracting the most probable energy loss for each counter in the sum. This energy is included in the reconstructed muon energy.

$E_{\mu}$ at the event vertex is reconstructed in two parts: energy deposited in the target calorimeter (typically less than $10 \mathrm{GeV}$ ) and remaining energy that is measured

${ }^{1}$ The definition of one MIP used in NuTeV is the following: the mean energy deposited by a $77 \mathrm{GeV}$ muon in one counter determined using a truncated mean procedure see [6]. in the downstream toroid spectrometer. Energy deposited in the target calorimeter includes the muon energy within the shower region (discussed above) and energy deposited beyond the shower region before the muon exits the calorimeter. The latter is determined from the pulse height of energy deposited in each counter by the muon. For small pulse heights, (< 5 MIPs), the muon energy loss is assumed to arise from ionization processes and is converted to $\mathrm{GeV}$ using an $E_{\mu}$ dependent conversion function which was optimized using GEANT to reproduce both the most probable value and the width of this component of the energy loss (see [6]). For larger pulse heights ( $>5$ MIPs), the energy is assumed to have a contribution from catastrophic processes (i.e. bremsstrahlung, pair production). Therefore, the additional amount above 5 MIPs is converted to GeV using a calibration constant determined from the calorimeter's electromagnetic response. The contribution to the muon energy resolution from determination of energy loss in the target is small for high energy muons and dominated by a long tail produced by catastrophic energy loss processes.

Muons that enter the toroid spectrometer are focused and tracked through the spectrometer where they are momentum analyzed. Figure 3 shows the momentum resolution for the toroid spectrometer determined using test beam muons. The Gaussian contribution is dominated by multiple Coulomb scattering (MCS) and is independent of momentum $(\sim 11 \%)$. The high-end tail is due to catastrophic energy loss processes. Test beam data are used to parameterize the resolution functions using a fit of the form

$$
\begin{aligned}
\frac{\Delta\left(1 / P_{\mu}\right)}{\left(1 / P_{\mu}\right)} & =\exp \left[-\frac{1}{2} \frac{x^{2}}{\sigma_{\text {lead }}^{2}\left(P_{\mu}\right)}\right] \\
& +R_{\text {tail }}\left(P_{\mu}\right) \exp \left[-\frac{1}{2} \frac{\left(x-x_{\text {tail }}\left(P_{\mu}\right)\right)^{2}}{\sigma_{\text {tail }}^{2}\left(P_{\mu}\right)}\right],
\end{aligned}
$$

where $\sigma_{\text {lead }}\left(P_{\mu}\right)$ is the width of the leading Gaussian contribution due to MCS, and $R_{\text {tail }}\left(P_{\mu}\right), \sigma_{\text {tail }}\left(P_{\mu}\right)$ and $x_{\text {tail }}\left(P_{\mu}\right)$ are the normalization coefficient, the width and the offset of the asymmetric tail of the resolution function. These parameters are found to have small energy dependences. The width of the leading Gaussian is parameterized with the following function

$$
\sigma_{\text {lead }}\left(P_{\mu}\right)=\sqrt{A^{2}+\left(B P_{\mu}\right)^{2}}
$$

where $A=0.10$ and $B=4.2 \times 10^{-3} . \quad R_{\text {tail }}\left(P_{\mu}\right)$, $\sigma_{\text {tail }}\left(P_{\mu}\right)$, and $x_{\text {tail }}\left(P_{\mu}\right)$ are parameterized with linear 


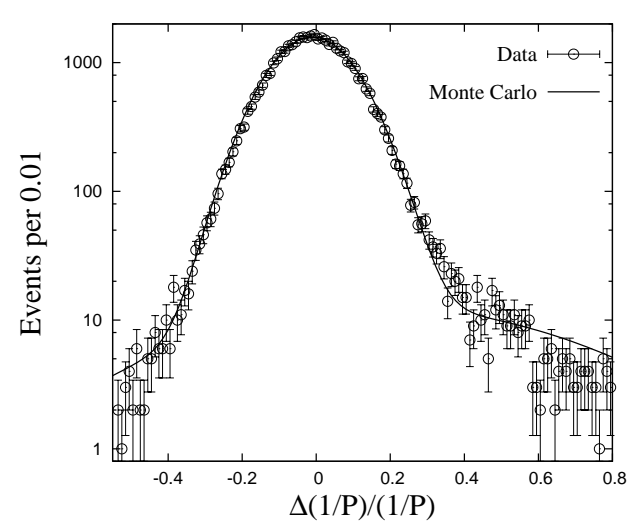

FIG. 3. Test beam data (points) with muon momentum of $100 \mathrm{GeV}$ measured in the toroid spectrometer compared with double Gaussian fit parameterization curve.

functions. For a muon entering the toroid with momentum $P_{\mu}=100 \mathrm{GeV}$ the resolution function parameters have the following values $\sigma_{\text {lead }}(100)=0.11, \sigma_{\text {tail }}(100)=$ $0.47, x_{\text {tail }}(100)=0.18$, and $R_{\text {tail }}(100)=0.079$.

Muon angle is determined using the track vector in the target calorimeter extrapolated back to the event vertex. Resolution on muon angle is dominated by multiple scattering in the target and is determined from GEANT hitlevel simulation of the detector. Angular resolution (in $m R a d)$ is parameterized as

$$
\Delta \theta_{\mu}=\left(96.8+0.87 L+0.24 E_{\mathrm{HAD}}\right) / P_{\mu}
$$

for a muon with momentum $P_{\mu}$ (in $\mathrm{GeV}$ ) where $L$ is the track length in the target in units of counters, and $E_{\mathrm{HAD}}$ is the shower energy in $\mathrm{GeV}$. The small dependence on hadron energy comes from an $E_{\mathrm{HAD}}$ dependent cut excluding tracking chambers near the event vertex due to the presence of additional hits in the shower region.

\section{B. Data Selection}

The following criteria were used to select the events for the cross section sample:

(1) Event containment: transverse vertex within $125 \mathrm{~cm}$ from detector center and longitudinal vertex at least four counters beyond the upstream end of the detector and beginning at least twenty counters from the downstream end.

(2) Reconstructed muon energy: A single muon in the event with minimum energy, $E_{\mu}>15 \mathrm{GeV}$.

(3) Reconstructed muon track in toroid: transverse position within, $15<$ radius $<160 \mathrm{~cm}$ upon entering; minimum penetration of track to the second chamber station; and minimum fraction of integrated track in steel $\geq 80 \%$.

(4) Reconstructed hadronic energy: $E_{H A D}>10 \mathrm{GeV}$.

(5) Reconstructed neutrino energy: $30<E_{\nu}<$ $360 \mathrm{GeV}$, is required to ensure that the flux is well understood and the muon momentum well reconstructed.

(6) Reconstructed $Q^{2}: Q^{2}>1 \mathrm{GeV}^{2}$, is required to ensure that non-perturbative contributions in our cross section model are small.

(7) Reconstructed Bjorken-x: $x<0.8$. Data at higher $x$ are excluded because smearing effects are large and our model is not well constrained in this region.

The final samples passing these cuts contained $8.6 \times 10^{5}$ neutrino $\left(\nu_{\mu}\right)$ and $2.4 \times 10^{5}$ anti-neutrino $\left(\bar{\nu}_{\mu}\right)$ events.

The differential cross section is determined from the differential number of events and the flux, $\Phi(E)$, at a given neutrino energy,

$$
\frac{d^{2} \sigma_{i j k}^{\nu(\bar{\nu})}}{d x d y} \propto \frac{1}{\Phi\left(E_{i}\right)} \frac{\Delta N_{i j k}^{\nu(\bar{\nu})}}{\Delta x_{j} \Delta y_{k}} .
$$

The quantity $\frac{d^{2} \sigma_{i j k}^{\nu(\bar{\nu})}}{d x d y}$ represents the average differential cross section in bin $i j k$. The absolute flux was not measured in NuTeV. The flux was normalized so that the average $\mathrm{NuTeV}$ total cross section from $30-200 \mathrm{GeV}$ is equal to the world average value (see section III C).

The number of events in a given bin, $N_{i j k}^{\nu(\bar{\nu})}$, must be corrected for bin acceptance due to detector geometry and kinematic cuts, and for bin migration caused by experimental resolution. The cross section measured in Equation 4 is corrected to the bin-center value

$$
\frac{d^{2} \sigma^{\nu(\bar{\nu})}}{d x d y}\left(E_{i}^{c}, x_{j}^{c}, y_{k}^{c}\right)=\frac{d^{2} \sigma_{i j k}^{\nu(\bar{\nu})}}{d x d y} \times \frac{S\left(E_{i}^{c}, x_{j}^{c}, y_{k}^{c}\right)}{\bar{S}_{i j k}}
$$


where $S\left(E_{i}^{c}, x_{j}^{c}, y_{k}^{c}\right)$ is the differential cross section evaluated at the bin-center values $E_{i}^{c}, x_{j}^{c}, y_{k}^{c}$ and $\bar{S}_{i j k}$ is the average value of the cross section determined from the integral over the bin

$$
\bar{S}_{i j k}=\frac{1}{\Delta x_{j} \Delta y_{k}} \int_{x_{j}}^{x_{j}+1} \int_{y_{k}}^{y_{k}+1} \frac{d^{2} \sigma\left(E_{i}, x, y\right)}{d x d y} d x d y .
$$

This correction is calculated by integration of the Monte Carlo model (described in section III D) and is most important at low and high $x$.

\section{Neutrino Flux}

The neutrino (and antineutrino) relative flux as a function of energy is determined using the "fixed $\nu_{o}$ " method [4]. Integrating the differential cross section given in eq. 1 over $x$ gives

$$
\frac{d \sigma}{d \nu}=A\left(1+\frac{B}{A} \frac{\nu}{E}-\frac{C}{A} \frac{\nu^{2}}{2 E^{2}}\right)
$$

where $\nu=E_{\mathrm{HAD}}$ and $E$ is the incident neutrino energy. The coefficients are given by,

$$
\begin{gathered}
A=\frac{G_{F} M}{\pi} \int F_{2}(x) d x \\
B=-\frac{G_{F} M}{\pi} \int\left(F_{2}(x) \mp x F_{3}(x)\right) d x \\
C=B-\frac{G_{F} M}{\pi} \int F_{2}(x) R_{\text {TERM }} d x
\end{gathered}
$$

where, $R_{\text {TERM }}=\left(\frac{1+\frac{2 M x}{\nu}}{1+R_{L}}-\frac{M x}{\nu}-1\right)$, depends on the longitudinal structure function, $R_{L}(x)$. Multiplying both sides of eq. 5 by the flux $\Phi(E)$ gives the number of events

$$
\frac{d N}{d \nu}=\Phi(E) A\left(1+\frac{B}{A} \frac{\nu}{E}-\frac{C}{A} \frac{\nu^{2}}{2 E^{2}}\right) .
$$

As $\nu \rightarrow 0$ the cross section (eq. 5) is independent of energy and therefore the number of events at low $\nu$ is proportional to the flux, $\frac{d N}{d \nu} \rightarrow \Phi(E) A$. To minimize the statistical uncertainty, data up to $\nu=20 \mathrm{GeV}$ are included in our flux sample. Therefore a correction is applied to account for the energy dependence as discussed below. Substituting for the coefficient $\mathrm{C}$, the relative flux is then given by

$$
\Phi(\mathrm{E})=\int_{0}^{\nu_{0}} \frac{\frac{d N(\mathrm{E})}{d \nu}}{1+\left\langle\frac{B}{A}\right\rangle\left(\frac{\nu}{E}-\frac{\nu^{2}}{2 E^{2}}\right)+\frac{\nu^{2}}{2 E^{2}} \frac{\int F_{2}(x) R_{\mathrm{TERM}}}{\int F_{2}(x)}} d \nu .
$$

The term $\int F_{2}(x) R_{\text {TERM }} / \int F_{2}(x)$ is obtained by integrating the structure functions, calculated using our Monte Carlo model.

In reference [4] it was assumed that the coeficients $A$ and $B$ do not depend on $\nu$. The integration over $x$ at fixed $\nu$ gives an implicit $Q^{2}$ dependence, $Q^{2}=2 M x \nu$. For different values of $\nu$ the integral will be over different ranges in $Q^{2}$. A $\nu$-dependent scaling violations correction is applied to account for this effect. The correction is obtained by integrating the structure functions $F_{2}$ and $x F_{3}$ over $x$ at fixed $\nu$, using our Monte Carlo model. As described below, this correction shifts the measured value of $\frac{B}{A}$ and has a small effect on the extracted flux.

The flux data sample consists of events which are contained in the detector, have a well constructed muon with minimum energy $E_{\mu}>15 \mathrm{GeV}$, neutrino energy in the range $30<E<360 \mathrm{GeV}$, and $E_{\mathrm{HAD}}<20 \mathrm{GeV}$. The $E_{\mathrm{HAD}}$ cut makes this sample orthogonal to the sample used to measure the cross sections. The data are corrected for acceptance and detector effects using our Monte Carlo model. Corrections were also applied to remove QED radiative effects using [12] and for the charm production threshold using a leading-order slow rescaling model with the charm mass parameter, $m_{c}=1.40 \pm 0.18$ (see Appendix). Radiative corrections range from $-2 \%$ at $30 \mathrm{GeV}$ to $4 \%$ at $290 \mathrm{GeV}$. The charm production correction decreases with energy and is about $5 \%$ for the flux sample at $30 \mathrm{GeV}$.

The coefficient $\frac{B}{A}$ is determined from a fit to $\frac{d N}{d \nu}$ over the range $5<\nu<20 \mathrm{GeV}$. Limiting $\nu$ to above $5 \mathrm{GeV}$ reduces the contribution from quasi-elastic and resonance processes, which are difficult to model. The coefficients of the fit parameters $A$ and $B$ are modified to remove the $\nu$ dependence due to scaling violations. The fit is performed for each energy and the average value of $\left\langle\frac{B}{A}\right\rangle$ over all energy bins is used. We obtain $\left\langle\frac{B}{A}\right\rangle=-0.34 \pm 0.04$ for neutrinos and $-1.68 \pm 0.03$ for anti-neutrinos. The effect of scaling violations is to shift the average value of $\left\langle\frac{B}{A}\right\rangle$ by +0.13 for neutrinos and +0.03 for anti-neutrinos. The effect on the extracted neutrino flux ranges from $3.5 \%$ at $35 \mathrm{GeV}$ and is negligible above $120 \mathrm{GeV}$ for neutrinos. For anti-neutrinos the correction to $\left\langle\frac{B}{A}\right\rangle$ is small and the 
effect on the flux is less than $0.8 \%$ for all energies. Because the scaling violations correction is calculated from a model we estimate a systematic uncertainty in $\left\langle\frac{B}{A}\right\rangle$ to be $0.05(0.04)$ for neutrinos(anti-neutrinos). This theoretical uncertainty is obtained by using an alternative model [13] to evaluate the scaling violations and to extract $\left\langle\frac{B}{A}\right\rangle$. The value of $\frac{B}{4}$ computed directly from the NuTeV cross section model (at $\nu=20 \mathrm{Gev}$ ) gives -0.29 for neutrinos and -1.66 for antineutrinos which compares well with the value computed for the alternative cross section model (Bodek-Yang) from reference [13] which gives -0.28 and -1.68 , respectively.

The total cross section is used to normalize the flux. A sample of events are selected which are contained in the detector and have well constructed muon with minimum energy $E_{\mu}>15 \mathrm{GeV}$. The total neutrino(antineutrino) cross section is

$$
\frac{\sigma^{\nu(\bar{\nu})}(E)}{E}=\frac{N^{\nu(\bar{\nu})}(E)}{E \cdot \Phi(\mathrm{E})}
$$

where $N^{\nu(\bar{\nu})}(E)$ is the number of total cross section events, corrected for acceptance and detector effects, and $\Phi(\mathrm{E})$ is the relative flux.

The flux is normalized using the world average neutrino cross section from $30-200 \mathrm{GeV}[8]$.

$$
\frac{\sigma^{\nu}}{E}=0.677 \pm 0.014 \times 10^{-38} \frac{\mathrm{cm}^{2}}{\mathrm{GeV}}
$$

The cross section normalization uncertainty, $2.1 \%$, arises from the quoted errors on the world average absolute neutrino cross section. Figure 4 shows the energy dependence of the total cross section, $\frac{\sigma}{E}$. The total neutrino and antineutrino cross sections are linear with energy to better than $2 \%$ over our energy range. The relative $\bar{\nu}$ to $\nu$ cross section, $r=\frac{\sigma^{\nu}}{\sigma^{\nu}}$, can be measured from $\mathrm{NuTeV}$ data alone which gives a value of $r=0.505 \pm 0.0018$ (stat) \pm 0.0029 (syst). This measurement is the most accurate determination to date and it agrees with the previous world average value of $r=$ $0.499 \pm 0.007$.

\section{Cross Section Extraction}

The Monte Carlo simulation, used to account for acceptance and resolution effects, requires an input cross section model which is iteratively tuned to fit our data. Our cross section model, described in the Appendix, is

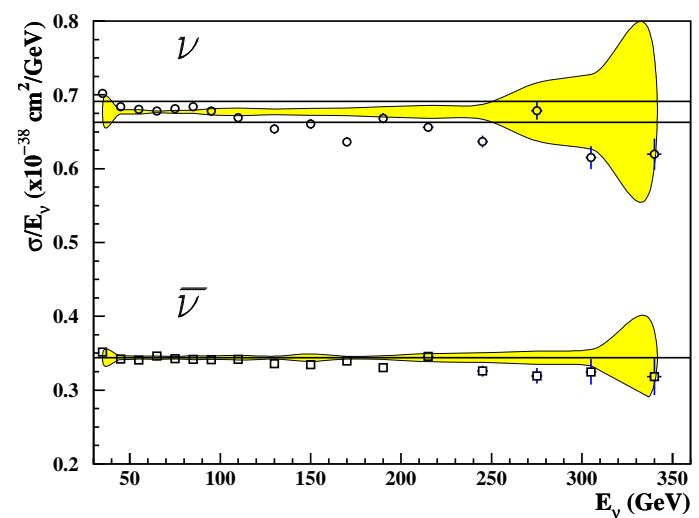

FIG. 4. Total cross section, $\frac{\sigma}{E}$, as a function of energy for neutrino (circles) and anti-neutrino (squares) interactions. The error bars are statistical uncertainty and the yellow band shows the size of the systematic uncertainty.

based on a leading order prescription from Buras and Gaemers [9] that is modified to incorporate higher-order corrections such as $R_{L}\left(x, Q^{2}\right)$, charm mass, and highertwist effects which are important at low $Q^{2}$. Cross section data are fit to determine empirically a set of parton distribution functions. To model regions at the edge of our data sensitivity we use additional input to constrain the cross section. At high- $x$ and low $Q^{2}$ we model highertwist contributions following reference [10] by incorporating charged-lepton data in the fit. At low $x$ and low $Q^{2}$ (below $1.35 \mathrm{GeV}^{2}$ ), where the Buras-Gaemers parameterization is not well behaved, the shape of GRV94LO $[11]$ is used.

The cross section, flux, and the model parton distribution functions $(P D F \mathrm{~s})$ are obtained by a reiterative extraction and refitting proceedure. An initial set of model parameters from CCFR [3] are used to extract an initial flux and cross section. From these we perform a fit to obtain a set of $P D F$ s which are then used to extract a new flux and cross section. The proceedure is reiterated until the relative change in cross section value from one iteration to the next averaged over all the data points is less than $0.1 \%$ (this occurs within three iterations). New radiative corrections calculated from [12] are computed for each new cross section fit. After the final iteration, we exclude kinematic bins where the number of events generated in that bin account for less than $20 \%$ of the 
events. This reduces the contribution from the high $E_{\mu}$ tail where smearing dominates the distribution.

Figure 5 shows a comparison of the distributions of the three kinematic variables measured in data with those determined from the Monte Carlo model. The data versus model $\chi^{2}$ including systematic uncertainties is $\chi^{2} / d o f=2225 / 2599$. If full point-to-point data correlations are included in the $\chi^{2}$ the fit quality worsens to $\chi^{2} / d o f=3534 / 2599$. (The data correlation matrix is discussed in Section IIIF). The inclusion of the extremely precise charged-lepton data in our model fit (see Appendix) has the effect of systematically pulling the $Q^{2}$ dependence of the model into agreement with the charged-lepton data and worsens the quality of the model fit with $\mathrm{NuTeV}$ data. Alternative models based on global parton distribution fits give a significantly poorer $\chi^{2}$ with our data; the Bodek-Yang model from reference [13] gives $\chi^{2}=5969 / 2599$ and TRVFS(MRST99) [14] gives $\chi^{2}=5000 / 2599$. Model sensitivity in the cross section measurement is small (see section III F).

\section{E. Results}

Figures 6-8 show the extracted $\nu-F e$ and $\bar{\nu}-F e$ cross sections plotted as a function of $y$ for a representative sample of $x$ bins at neutrino energies of $65 \mathrm{GeV}, 150 \mathrm{GeV}$, and $245 \mathrm{GeV}$ respectively. The $\mathrm{NuTeV}$ data are compared with measurements from CCFR [3] and at lower energies with CDHSW data [2]. The curve shown is the parameterization fit to the $\mathrm{NuTeV}$ data. The three data sets are in reasonable agreement in both level and shape at low and moderate $x$. There are differences in $\mathrm{NuTeV}$ and CCFR cross sections at $x>0.40$ where CCFR's measurement for both $\nu$ and $\bar{\nu}$ cross sections are consistently below the $\mathrm{NuTeV}$ result over the entire energy range. The level difference in the cross sections for these bins at a given $x$ is constant over the full $y$ range of the data The difference in the neutrino cross sections are $4 \pm 1 \%$ at $x=0.45,9 \pm 2 \%$ at $x=0.55$, and increases with $x$ up to $18 \pm 2 \%$ at $x=0.65$, (and similarly for antineutrinos). This discrepancy and its probable source are discussed in Section V.

\section{F. Systematic Uncertainties}

We evaluated the contribution from seven experimental systematic uncertainties on the cross section measure-
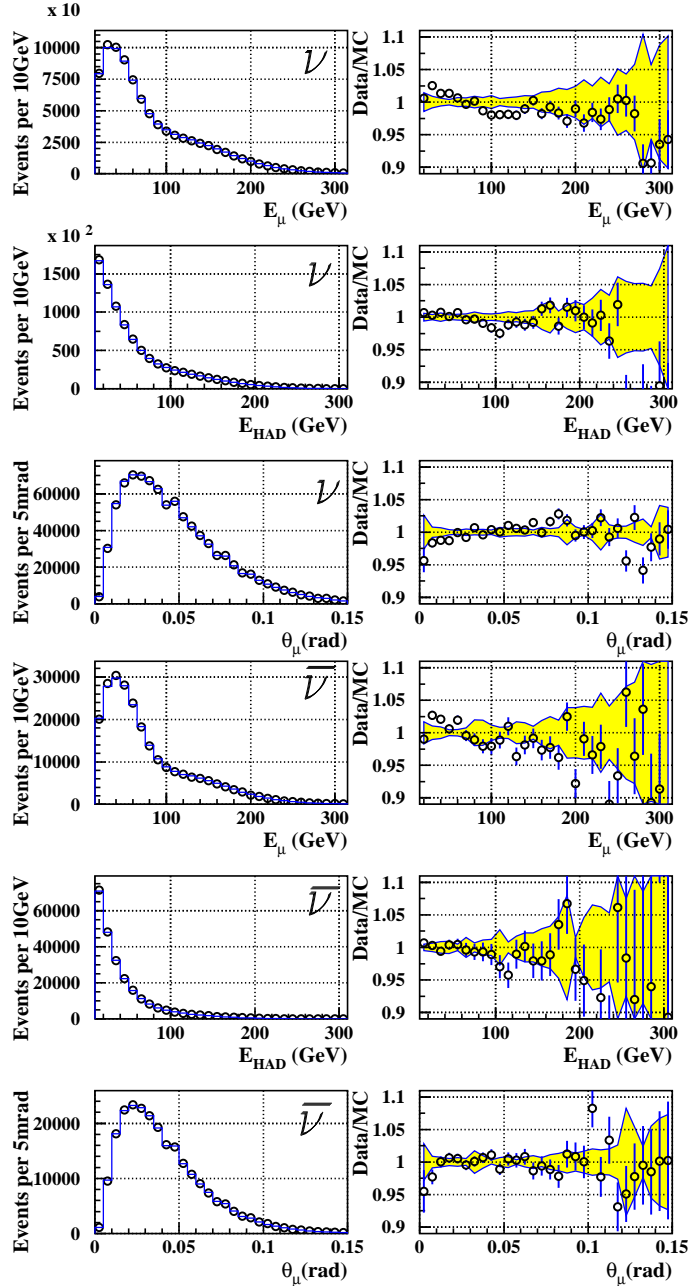

FIG. 5. Comparison of data distributions for kinematic variables, $E_{\mu}, E_{H A D}$, and $\theta_{\mu}$ with Monte Carlo simulation for neutrinos (top) and anti-neutrinos (bottom). The points are data and the curve is the Monte Carlo model. Ratio of data to Monte Carlo is plotted at the right of each distribution. The error bars are statistical uncertainty and the yellow band shows the size of the systematic uncertainty. 


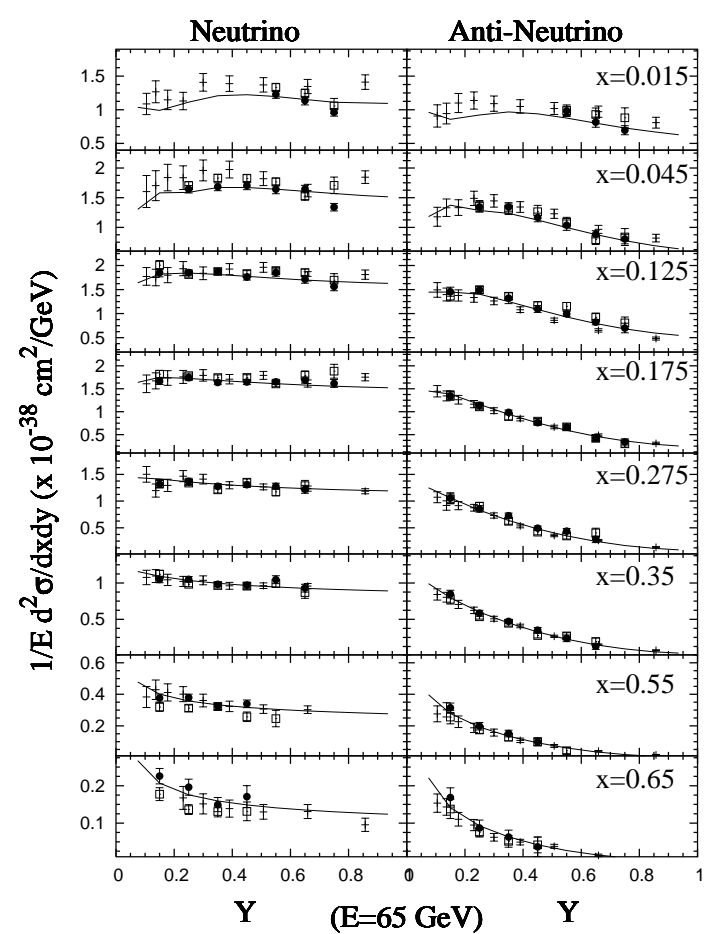

FIG. 6. Differential cross sections in $x$ bins for neutrinos (left) and anti-neutrinos (right) at $E=65 \mathrm{GeV}$. Points are $\mathrm{NuTeV}$ (filled circles), CCFR (open squares), and CDHSW (crosses). Error bars show statistical and systematic errors in quadrature. Solid curve shows fit to NuTeV data. ( $x=0.08$, $0.225,0.45$, and 0.75 bins are not shown).

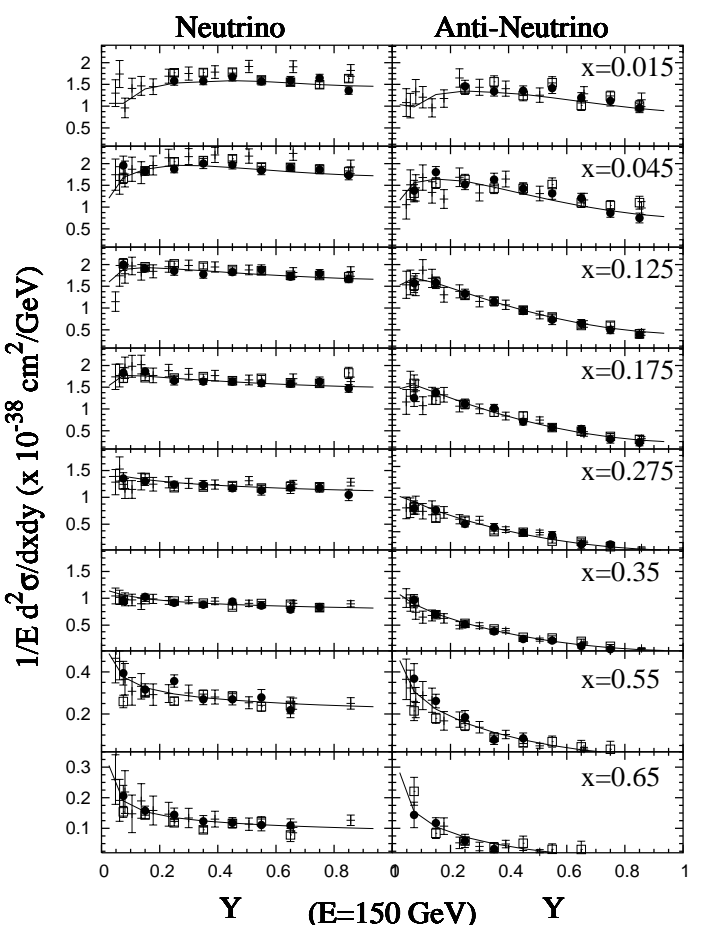

FIG. 7. Differential cross sections in $x$ bins for neutrinos (left) and anti-neutrinos (right) at $E=150 \mathrm{GeV}$. Points are $\mathrm{NuTeV}$ (filled circles), CCFR (open squares), and CDHSW (crosses). Error bars show statistical and systematic errors in quadrature. Solid curve shows fit to NuTeV data. ( $x=0.08$, $0.225,0.45$, and 0.75 bins are not shown). 


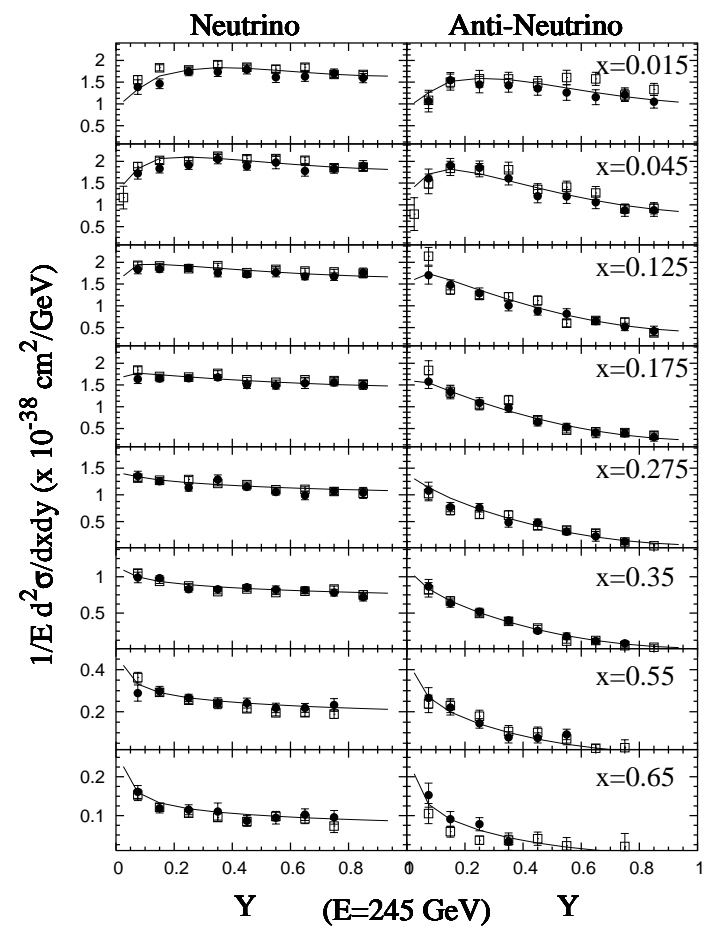

FIG. 8. Differential cross sections in $x$ bins for neutrinos (left) and anti-neutrinos (right) at $E=245 \mathrm{GeV}$. Points are $\mathrm{NuTeV}$ (filled circles), CCFR (open squares), and CDHSW (crosses). Error bars show statistical and systematic errors in quadrature. Solid curve shows fit to NuTeV data. $(x=0.08$, $0.225,0.45$, and 0.75 bins are not shown). ment error. These include uncertainties in the following: muon and hadron energy scales, muon and hadron energy smearing, the charm mass value $m_{c}$ and $\frac{B}{A}$ (both used in the flux determination), and the cross section model which is used to perform acceptance corrections. Each uncertainty is evaluated separately and then propagated through the fitting proceedure. The contribution to the cross section uncertainty from each systematic error (except the muon momentum smearing model which is described below) is evaluated by re-extracting the cross section with the value of the systematic parameter varied alternately by $\pm 1 \sigma$. The symmetrized difference in each cross section point is taken to be the $1 \sigma$ systematic error due to the uncertainty in the parameter.

The largest experimental systematic uncertainties are due to the muon and hadron energy scale which for $\mathrm{NuTeV}$ are $0.7 \%$ and $0.43 \%$ respectively. The neutrino and antineutrino fluxes are sensitive to the charm mass value $m_{c}$, used in the charm production model (see Appendix), and the value of $\frac{B}{A}$ used to correct the flux data sample. The uncertainty in the charm mass parameter is taken to be $\delta m_{c}=0.18$, which is obtained from the weighted average of leading-order experimental measurements [25], [26]. The values of $\frac{B}{A}$ for neutrino and antineutrino cross sections and their uncertainties are obtained from fits to the $\mathrm{NuTeV}$ flux sample data as described in III C. An uncertainty of $2.1 \%$ in the absolute flux determination arises from the normalization to the world average neutrino cross section. This is treated separately as an overall normalization uncertainty in the cross section.

Detector resolution functions for muon and hadron energy reconstruction also contribute to the systematic uncertainty since a different smearing model will generate different acceptance corrections from our Monte Carlo. The hadron energy response was determined from a fit to calibration beam data [6]. The response function is varied using the one sigma error from this fit as described above. The muon momentum smearing model is given by Equation 2. An estimate of the uncertainty due to the model is obtained using two different functional forms for the parameters of the leading Gaussian: the default model (Equation 3) and a second model which assumes a linear dependence with energy. Although both models describe the test beam data reasonably well their extrapolations differ at higher energies $\left(p_{\mu}>200 \mathrm{GeV}\right)$. The al ternative models are used to re-extract the flux and cross section. The uncertainty is taken to be the point-to-point difference between the extractions. The flux is manifestly 
independent of the muon angle smearing model and the cross section is highly insensitive. We, therefore, neglect this systematic uncertainty.

To estimate the uncertainty from the cross section model we vary the errors in the model fit parameters (see Appendix) by one sigma from their fit values. The resulting uncertainties on the cross section are smaller than the statistical precision on a Monte Carlo sample with approximately twenty times the data statistics. The effect of the model uncertainty on the cross section error is very small and is neglected.

The NuTeV data are presented along with a full pointto-point covariance matrix that provides the correlation coefficient between any two cross section data points. We have found that these correlations are large for neighboring bins. Previous measurements by [2] and [3] did not provide such a data correlation matrix. The covariance matrix, $M_{\alpha \beta}$, is given by

$$
M_{\alpha \beta}=\sum_{i}^{7} \delta_{i \mid \alpha} \delta_{i \mid \beta}
$$

where $\delta_{i \mid \alpha}$ is the $1 \sigma$ shift in data point $\alpha$ due to systematic uncertainty $i$. The $2.1 \%$ flux normalization uncertainty can be included in the covariance matrix by adding a term

$$
M_{\alpha \beta}^{\prime}=M_{\alpha \beta}+(0.021)^{2}{\frac{d^{2} \sigma}{d x d y}}_{\alpha}^{D}{\frac{d^{2} \sigma^{D}}{d x d y_{\beta}}}^{D}
$$

The statistical uncertainty is added in quadrature to the diagonal elements of the data covariance matrix.

Separate data vectors and covariance matrices are obtained for the neutrino and antineutrino cross sections. There are $1423\left(N_{D A T A}^{\nu}=1423\right)$ neutrino and 1195 $\left(N_{D A T A}^{\bar{\nu}}=1195\right)$ antineutrino data points. A $\chi^{2}$ with respect to a theoretical model can be calculated using

$$
\begin{aligned}
& \chi^{2}= \\
& =\sum_{\alpha, \beta=1}^{N_{D A T A}^{\nu}}\left[{\frac{d^{2} \sigma^{t h}}{d x d y}}_{\alpha}^{t h}-{\frac{d^{2} \sigma}{d x d y}}_{\alpha}{ }^{D}\right]\left(\mathbf{M}_{\nu}^{-1}\right)_{\alpha \beta}\left[{\frac{d^{2} \sigma^{t h}}{d x d y}}_{\beta}-{\frac{d^{2} \sigma^{D}}{d x d y}}_{\beta}\right]
\end{aligned}
$$

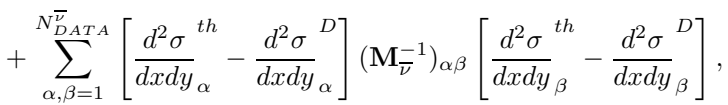

where ${\frac{d^{2} \sigma}{d x d y}}_{\alpha}^{D}$ is the measured differential cross section and ${\frac{d^{2} \sigma}{d x d y}}_{\alpha}^{t h}$ is the model prediction for data point $\alpha$.

\section{STRUCTURE FUNCTIONS}

Structure functions, $F_{2}\left(x, Q^{2}\right)$ and $x F_{3}\left(x, Q^{2}\right)$, can be determined from fits to linear combinations of the neutrino and antineutrino differential cross sections. The sum of the differential cross sections can be expressed as

$$
\begin{aligned}
& \frac{d^{2} \sigma^{\nu}}{d x d y}+{\frac{d^{2} \sigma^{\bar{\nu}}}{d x d y}}^{\nu}=\frac{G_{F}^{2} M E}{\pi}\left[2 \left(1-y-\frac{M x y}{2 E}\right.\right. \\
+ & \left.\left.\frac{y^{2}}{2} \frac{1+4 M^{2} x^{2} / Q^{2}}{1+R_{L}}\right) F_{2}+y\left(1-\frac{y}{2}\right) \Delta x F_{3}\right] .
\end{aligned}
$$

where $F_{2}$ is the average of $F_{2}^{\nu}$ and $F_{2}^{\bar{\nu}}$. The last term is proportional to the difference in $x F_{3}$ for neutrino and antineutrino probes, $\Delta x F_{3}=x F_{3}^{\nu}-x F_{3}^{\bar{\nu}}$, which at leading order is $4 x(s-c)$, (assuming symmetric $s$ and $c$ seas). Cross sections are corrected for the excess of neutrons over protons in the iron target $(5.67 \%)$ so that the presented structure functions are for an isoscalar target. A correction was also applied to remove QED radiative effects [12]. To extract $F_{2}\left(x, Q^{2}\right)$ we use $\Delta x F_{3}$ from a NLO QCD model as input (TRVFS [14]). The input value of $R_{L}\left(x, Q^{2}\right)$ comes from a fit to the world's measurements [15]. The NuTeV measurement of $F_{2}\left(x, Q^{2}\right)$ on an isoscalar-iron target is shown in Figure 9 . The structure function, $F_{2}\left(x, Q^{2}\right)$ is compared with previous measurements from CDHSW [2] and CCFR [3].

The difference of neutrino and anti-neutrino differential cross sections is proportional to the structure function $x F_{3}\left(x, Q^{2}\right)$,

$$
\frac{d^{2} \sigma^{\nu}}{d x d y}-\frac{d^{2} \sigma^{\bar{\nu}}}{d x d y}=\frac{2 G_{F}^{2} M E}{\pi}\left[y-\frac{y^{2}}{2}\right] x F_{3}^{A V G}\left(x, Q^{2}\right)
$$

where $x F_{3}^{A V G}=\frac{1}{2}\left(x F_{3}^{\nu}+x F_{3}^{\bar{\nu}}\right)$, and the difference between $F_{2}^{\nu}\left(x, Q^{2}\right)$ and $F_{2}^{\bar{\nu}}\left(x, Q^{2}\right)$ is assumed to be negligible. Figure 10 shows the $\mathrm{NuTeV}$ measurement of (6) $x F_{3}\left(x, Q^{2}\right)$ from fits to the cross section difference. The structure function, $x F_{3}\left(x, Q^{2}\right)$ is compared with previous measurements from CDHSW [2] and CCFR97 [16].

\section{DATA COMPARISONS}

At moderate $x$, this result agrees well with CCFR over the full energy and $y$ range of the data, both in level and in shape, and agrees in level with CDHSW. The CDHSW measurement has a known $Q^{2}$ shape difference with CCFR [3], and thus also with this result. There 


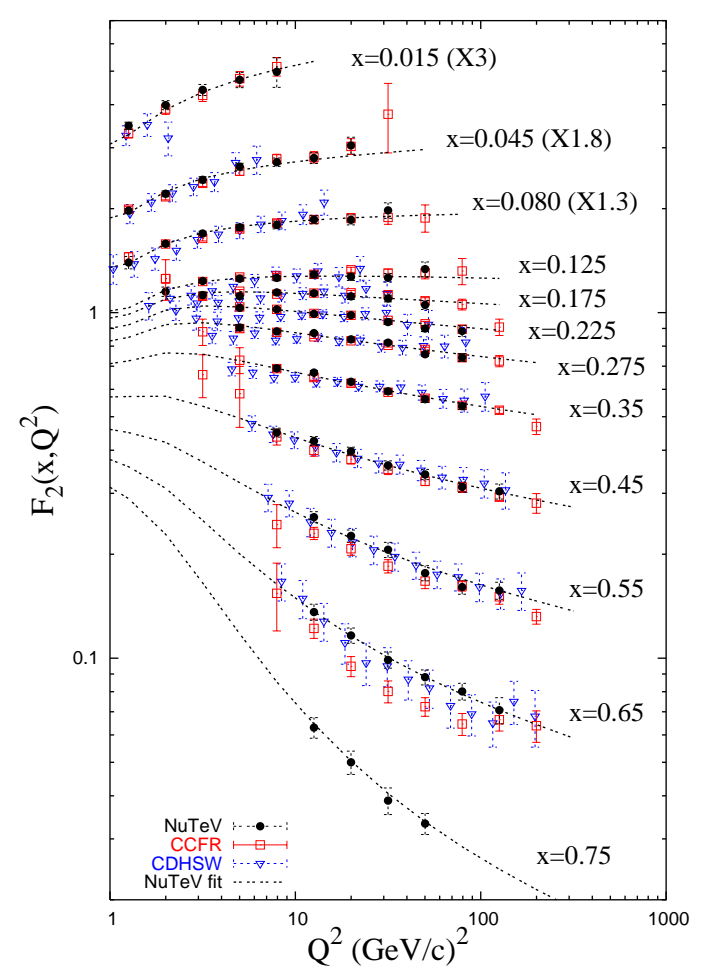

FIG. 9. NuTeV measurement of $F_{2}\left(x, Q^{2}\right)$ (solid circles) compared with previous $\nu$-Fe results; CCFR (open circles) and CDHSW (triangles). The data are corrected to an isoscalar (iron) target and for QED radiative effects as described in the text. The curve show the NuTeV model.

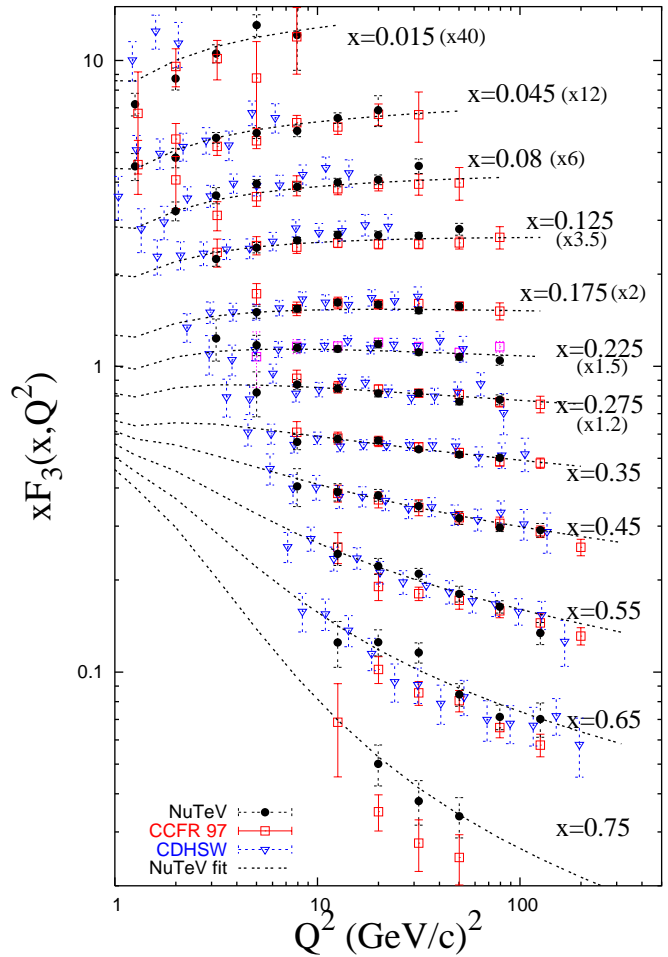

FIG. 10. $x F_{3}\left(x, Q^{2}\right) \mathrm{NuTeV}$ (solid circles) compared with previous measurements; CCFR97 (open circles) and CDHSW (triangles). The data are corrected to an isoscalar (iron) target and for QED radiative effects as described in the text. The curve shows the NuTeV model. 
are differences in $\mathrm{NuTeV}$ and CCFR at $x>0.40$ where CCFR's measurement for both neutrino and antineutrino cross sections are consistently below our result for all energies. This is suprising since NuTeV used the refurbished CCFR detector and the analysis methods used by the two experiments were very similar. We discuss below several sources which may contribute to the high- $x$ cross section difference.

We have determined that the largest single contribution to the discrepancy is due to a mis-calibration of the magnetic field map of the toroid in CCFR. NuTeV performed thorough calibrations of muon and hadron responses in the detector, including mapping the response over the detector active area and measuring the energy scale over a wide range of energies [6]. This allowed $\mathrm{NuTeV}$ to measure precisely the radial dependence of the magnetic field in the toroid. Both experiments used the same muon spectrometer; therefore, the model of the magnetic field could have a different overall normalization, but the radial dependence (which is determined by the geometry of the muon spectrometer) should be the same for both. CCFR used one high statistics muon test beam run aimed at a single point in the spectrometer to set the absolute energy scale and modeled the radial dependence of the magnetic field using POISSON [17]. NuTeV used ANSYS to model the field and compared the prediction to a precision test beam map data. The width of the residual fractional difference distribution over the 45 test beam points is the main contribution to the absolute muon energy scale uncertainty $(0.7 \%)$. If $\mathrm{NuTeV}$ uses the CCFR model the result is shifted to within 1.6 sigma agreement with CCFR at $x=0.65$. This accounts for $6 \%$ of the $18 \%$ difference at $x=0.65$. The field model differences can also be translated into an effective $0.8 \%$ difference in the muon energy scales by integrating the difference in the field models over the toroid.

The cross section model contributes an additional $\sim 3 \%$ to the discrepancy seen at $\mathrm{x}=0.65$. Both experiments determine acceptance corrections using an iterated fit to the cross section data. Because the measured cross sections are different, this necessarily requires that the respective cross section models reflect the data differences. For example, the NuTeV model is above CCFR by $\sim 20 \%$ at $\mathrm{x}=0.65$. We have extracted cross sections using CCFR cross section model [3] and find that this contributes $\sim 3 \%$ to the discrepancy seen at $\mathrm{x}=0.65$.

Other smaller sources for the difference come from muon and hadron energy smearing models which can also contribute to extracted cross section differences through acceptance corrections. We have determined that using the CCFR muon and hadron smearing models results in a difference of $\sim 2 \%$ at $x=0.65$. In NuTeV the hadron energy response was found to have a small nonlinearity due to the shower $\pi^{0}$ fraction dependence on energy [6]. This nonlinearity was taken into account in $\mathrm{NuTeV}$ but not in CCFR. The effect of incorporating the hadron energy scale nonlinearity is small and contributes mainly to the $Q^{2}$ dependence.

All together these three contributions account for about two thirds of the high- $x$ cross section difference seen. This brings the two measurements within 1.2 sigma agreement in the high- $x$ region.

Another significant difference in the two experiments is that, while both used wide-band beams, NuTeV's beam was sign-selected. In $\mathrm{NuTeV}$, neutrino and anti-neutrino data were taken using separate high-purity beams. This allowed $\mathrm{NuTeV}$ to run the detector's toroidal magnetic field polarity always set to focus the "right-sign" of muon produced in charged-current interactions. In CCFR, the beam had an $11 \%$ anti-neutrino component and neutrino and anti-neutrino data were taken simultaneously. The toroidal field polarity was reversed perodically to alternately focus either $\mu^{+}$or $\mu^{-}$. To obtain adequate antineutrino statistics CCFR ran approximately half the time in focussing mode for each muon sign. This has two effects on the CCFR analysis that are not present in NuTeV. First, the acceptance corrections are different depending on whether the toroid is set to focus or defocus the muon. Second, in defocussing mode acceptance corrections are larger and consequently need to be more accurately determined. Acceptance falls off rapidly with $y$ at high- $x$, especially in defocussing mode where low-energy and wide-angle muons are deflected outward and spend less time the toroid magnetic field. CCFR had no test beam data for defocussing mode and, while acceptance corrections were modeled with a field simulation, the smearing model was assumed to be the same in both modes. We speculate that this difference may also contribute to the discrepancy seen.

\section{THEORY COMPARISONS}

Figures 11 and 12 show a comparison of $\mathrm{NuTeV}$ and CCFR data with two theory models. The plot on Figure 11 shows the ratio of $F_{2}\left(x, Q^{2}\right)$ to the Thorne-Roberts variable-flavor scheme (TRVFS) NLO QCD model [14] using the MRST2001 NLO PDF set [18] for all $x$ bins. 


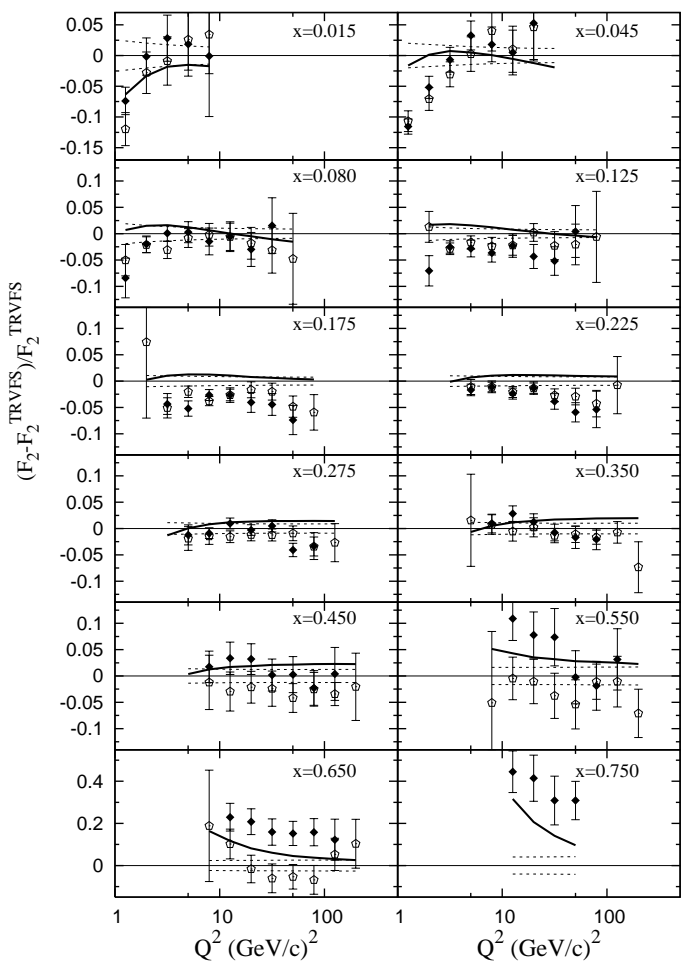

FIG. 11. $F_{2}\left(x, Q^{2}\right)$ fractional difference $\frac{F_{2}-F_{2}^{T R V F S}}{F_{2}^{T R V F S}}$ with respect to the TRVFS(MRST2001E) model. ${ }^{2}$ Data points are $\mathrm{NuTeV}$ (solid dots) and CCFR (open circles). Theory curves are ACOTFFS(CTEQ5HQ1) (solid line) and TRVFS(MRST2001E) $\pm 1 \sigma$ (dashed lines). Theory curves are corrected for target mass and nuclear effects.

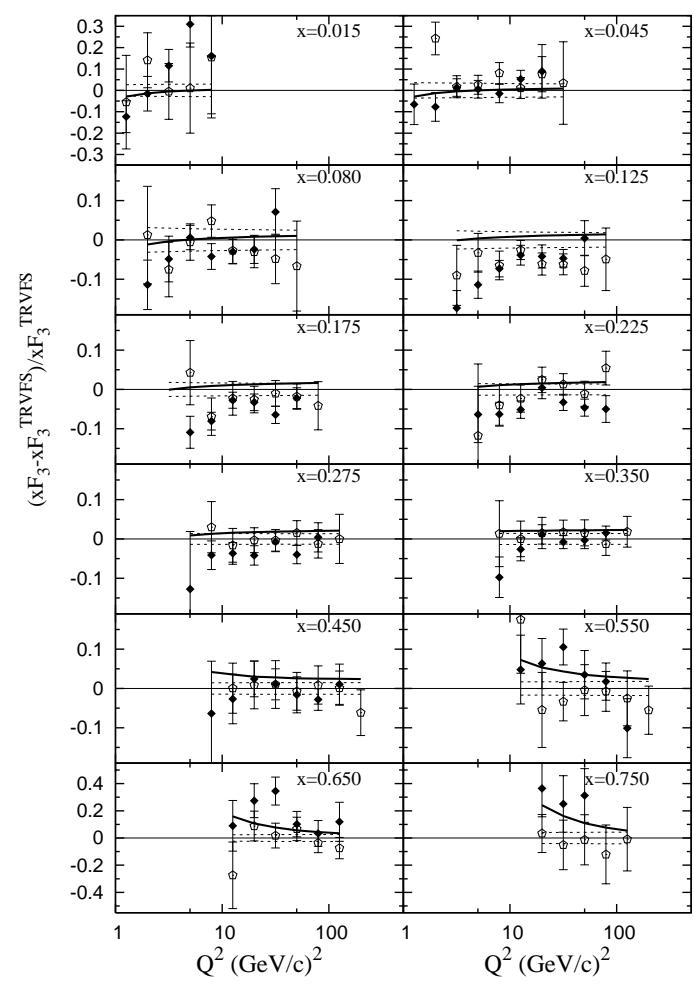

FIG. 12. $x F_{3}\left(x, Q^{2}\right)$ fractional difference $\frac{x F_{3}-x F_{3}^{T R V F S}}{x F_{3}^{T R V F S}}$ with respect to the TRVFS(MRST2001E) model. Data points are $\mathrm{NuTeV}$ (solid dots) and CCFR (open circles). Theory curves are ACOTFFS(CTEQ5HQ1) (solid line) and TRVFS(MRST2001E) $\pm 1 \sigma$ (dashed lines). Theory curves are corrected for target mass and nuclear effects. 
The $1 \sigma$ error band from the PDF set is also shown. The second theory curve shown on the plot is ACOT fixed-flavor scheme (ACOTFFS) NLO model [19] using CTEQ5HQ1 [20] PDFs. Similarily, the plot on Figure 12 shows the same comparison for $x F_{3}\left(x, Q^{2}\right)$. All theory models have been corrected for target-mass effects using the Georgi-Politzer model [21]. These are important at high- $x$ and low- $Q^{2}$. For example, they increase the theory prediction by about $5 \%$ at $x=0.65$ and $Q^{2}=15 \mathrm{GeV}^{2}$. To make a direct comparison, the theory curves are also corrected for nuclear target effects. A standard treatment of nuclear effects, which are not well determined for neutrino scattering, is to apply corrections measured in charged-leptons scattering from nuclear targets [4]. We use a multiplicative correction factor of the form [8]

$$
f(x)=1.10-0.36 x-0.28 e^{-21.9 x}+2.77 x^{14.4}
$$

obtained from a fit to charged-lepton scattering on nuclear targets. The correction is independent of $Q^{2}$ and is small at intermediate $x$ but is large at low and high $x$, $(10 \%$ at $x=0.015$ and increases from $7 \%$ at $x=0.45$ to $15 \%$ at $x=0.65)$

The data show reasonable agreement with both the TRVFS(MRST2001E) and the ACOTFFS(CTEQ5HQ1) NLO QCD calculations for most of the $x$ and $Q^{2}$ range. At low $x(x=0.015$ and $x=0.045)$ both $\mathrm{NuTeV}$ and CCFR results have different $Q^{2}$ dependence than theoretical predictions. At high- $x(x>0.5)$ our data are systematically above the theory predictions. Compared with the TRVFS(MRST2001E) model the data are 15$20 \%$ high at $x=0.65$ and $25-40 \%$ at $x=0.75$. The data are about $10 \%$ higher at $x=0.65$ and $15 \%$ at $x=0.75$ than the ACOTFFS(CTEQ5HQ1) model prediction. The $Q^{2}$ dependence of the high-x data is similar to the prediction from the ACOTFFS(CTEQ5HQ1) model. A different theoretical treatment of nuclear effects could make a sizable difference at small and large $x$. NuTeV perhaps indicates that neutrino scattering favors smaller nuclear effects at high- $x$ than are found in charged-lepton scattering. At small $x$, new theoretica calculations show that in the shadowing region the nuclear correction has $Q^{2}$ dependence $[22,23]$. The standard nuclear correction obtained from a fit to charged lepton data implies a suppression of $10 \%$ independent of $Q^{2}$ at $x=0.015$, while for $x=0.015$ reference [23] finds a suppression of $15 \%$ at $Q^{2}=1.25 \mathrm{GeV}^{2}$ and suppression of $3.4 \%$ at $Q^{2}=7.94 \mathrm{GeV}^{2}$. This effect somewhat improves agreement with data at low- $x$.

\section{DATA ACCESS}

The $\mathrm{NuTeV}$ neutrino and antineutrino cross sections and point-to-point covariance matrix can be downloaded from reference [24]. The tar file contains an unpacking routine and information on how to use the results.

[1] V. Barone, C. Pascaud, F. Zomer, Eur. Phys. J. C12 (2000) 243.

[2] P. Berge et. al, Z. Phys. C49 (1991) 187.

[3] U. K. Yang, Ph. D. Thesis, University of Rochester, (2001), UR-1583.

44] J. Conrad, M. H. Shaevitz, and T. Bolton, Rev. Mod. Phys. 70 (1998) 4.

[5] G. Sterman et. al, Rev. Mod. Phys. 67 (1995) 157.

[6] D. A. Harris et. al, Nucl. Instrum. Methods A447 (2000) 377.

[7] Finite element analysis software, http://www.ansys.com.

[8] W. Seligman, Ph. D. Thesis, Columbia University, Nevis 292 (1997).

[9] A. J. Buras and K. L. F. Gaemers, Nucl. Phys. B132 (1978) 2109.

[10] A. Bodek and U. K. Yang, Nucl. Phys. Proc. Suppl. 112 (2002) 70 .

[11] M. Gluck et. al (GRV collaboration), Z. Phys. C67, 433 (1995).

[12] D. Y. Bardin and V. A. Dokuchaeva, JINR-E2-86-260 (1986).

[13] Arie Bodek, Inkyu Park, Un-ki Yang, Nucl. Phys. Proc. Suppl. 139 (2005) 113-118. [arXiv:hep-ph/0411202].

[14] R. Thorne and R. Roberts, Phys. Lett. B421 (1998) 303. A. D. Martin et. al. Eur. Phys. J. C18 (2000) 117.

[15] L. W. Whitlow et. al, Phys. Lett. B250 (1990) 193.

[16] W. G. Seligman et. al, Phys. Rev. Lett. 79 (1997) 1213.

[17] CERN computer program POISSON; see R. Holsinger CERN Computer Center Program Library T604.

[18] A. D. Martin, R. G. Roberts, W. J. Stirling, R. S Thorne, Eur. Phys. J. C28 (2003) 455-473.

[19] M. A. G. Aivazis, J. C. Collins, F. I. Olness, and W. K. Tung, Phys. Rev. D50, 3102 (1994).

[20] H. L. Lai et. al., Eur. Phys. J. C12 (2000) 375-392.

[21] H. Georgi and H. D. Politzer, Phys. Rev. D14 (1976) 1829 .

[22] S.A. Kulagin, R. Petti, [arXiv:hep-ph/0412425].

[23] J. W. Qiu and I. Vitev, Phys. Lett. B587, (2004) 52 [arXiv:hep-ph/0401062]. 
24] http://arxiv.org/e-print/hep-ex/0509010, http://wwwnutev.phyast.pitt.edu/results_2005/nutev_sf.html.

[25] S. Rabinowitz et. al, Phys. Rev. Lett. 70 (1993) 134.

26] P. Vilain et. al, Eur. Phys. J. C11 (1999) 19; P. Astier et. al, Phys. Lett. B486 (2000) 35.

[27] A. Hawker et. al, Phys Rev Lett 80 (1998) 3715.

[28] L. W. Whitlow et. al, Phys. Lett. B282 (1992) 475.

[29] A. C. Benvenuti et. al, Phys. Lett. B223 (1989) 485; A C. Benvenuti et. al, Phys. Lett. B236 (1989) 592.

[30] M. Arneodo et. al, Nucl. Phys. B483 (1997) 3.

\section{APPENDIX: NUTEV CROSS SECTION MODEL}

The $\mathrm{NuTeV}$ cross section model is inspired by the LO parameterization prescribed by Buras and Gaemers in reference [9]. The leading order model is modified to include non-leading order effects from $R_{L}$, higher-twist contributions, and the charm mass as described below. Because the quark densities used in the model are obtained from fits to neutrino-iron scattering data, no external model for nuclear effects is required to describe the $\mathrm{NuTeV}$ data. The cross section model described here is the 'Born'-level neutrino DIS cross section for an isoscalar target. In addition, we correct for radiative effects using reference [12].

The neutrino isoscalar structure functions are given by

$$
\begin{aligned}
2 x F_{1}\left(x, Q^{2}\right) & =x u_{v}\left(x, Q^{2}\right)+x d_{v}\left(x, Q^{2}\right) \\
& +2\left[x u_{s}\left(x, Q^{2}\right)+x d_{s}\left(x, Q^{2}\right)+x s_{s}\left(x, Q^{2}\right)\right], \\
F_{2}\left(x, Q^{2}\right) & =2 x F_{1}\left(x, Q^{2}\right) \times \frac{1+R_{L}\left(x, Q^{2}\right)}{1+4 M^{2} x^{2} / Q^{2}}, \\
x F_{3}\left(x, Q^{2}\right) & =x u_{v}\left(x, Q^{2}\right)+x d_{v}\left(x, Q^{2}\right) .
\end{aligned}
$$

The CKM matrix elements are used in the above formula to account for the mixing between the quarks, even though they are not shown here. $R_{L}\left(x, Q^{2}\right)$ is obtained from an empirical fit to world data as described in reference [15]

$$
\begin{aligned}
R_{L}\left(x, Q^{2}\right) & =\frac{0.0635}{\ln \left(Q^{2} / 0.04\right)} \Theta\left(x, Q^{2}\right) \\
& +\frac{0.5747}{Q^{2}}-\frac{0.3534}{Q^{4}+0.09} \\
\Theta\left(x, Q^{2}\right) & =1+12\left(\frac{Q^{2}}{1+Q^{2}}\right)\left(\frac{0.125^{2}}{x^{2}+0.125^{2}}\right) .
\end{aligned}
$$

The charm production cross section is calculated using the slow rescaling model [4]. The Bjorken scaling variable, $x$, is rescaled

$$
x \rightarrow \xi=x \cdot\left(1+\frac{m_{c}^{2}}{Q^{2}}\right) .
$$

The charm production differential cross section is suppressed by the factor $1-\frac{m_{c}^{2}}{2 M E \xi}$. The value of the charm mass parameter used, $m_{c}=1.40 \pm 0.18$, is obtained from the weighted average of leading-order experimental measurements [25], [26]. 
To account for higher-twist effects at high $x$ and low $Q^{2}$ an empirical model is used to constrain the $Q^{2}$ dependence of the $P D F$ s by rescaling $x$ following reference [10].

$$
x \rightarrow \xi_{H T}=x \frac{Q^{2}+B_{H T}}{Q^{2}+A_{H T} \cdot x},
$$

where $A_{H T}$ and $B_{H T}$ are fit parameters in the model. The shape of GRV94L0 [11] $P D F$ s is used to extrapolate the model for $Q^{2}<1.35 \mathrm{GeV}^{2} / \mathrm{c}^{2}$. The normalizations are constrained by matching the $P D F$ s to the BurasGaemers $P D F$ s at $Q^{2}=1.35 \mathrm{GeV}^{2} / \mathrm{c}^{2}$.

The form for the parton distribution functions follow the Buras-Gaemers parameterization in reference [9]. The valence distributions are given by

$$
\begin{aligned}
& x u_{v}(x)=u_{t o t} \times\left[x^{E_{1}}(1-x)^{E_{2}}+A V_{2} x^{E_{3}}(1-x)^{E_{4}}\right] \\
& x d_{v}(x)=d_{\text {tot }} \times x u_{v}(x) \times(1-x),
\end{aligned}
$$

with parameters $A V_{2}, E_{1}, E_{2}, E_{3}$, and $E_{4}$. The $Q^{2}$ dependence for the valence is given by

$$
\begin{aligned}
E_{i} & =E_{i 0}+E_{i 1} \cdot s, i=1,4 \\
s & =\ln \left[\frac{\ln \left(\frac{Q^{2}}{A_{0}^{2}}\right)}{\ln \left(\frac{Q_{0}^{2}}{A_{0}^{2}}\right)}\right]
\end{aligned}
$$

where the reference $Q_{0}^{2}$ is set to $12.6 \mathrm{GeV}^{2} / \mathrm{c}^{2}$ and the QCD scale, $A_{0}$, is a parameter in the fit.

The constraints on the valence distributions include the normalization of the valence densities, which is determined from a variant of the Gross-Llewllyn-Smith sum rule, and the relative normalization between $u_{v}$ and $d_{v}$, which is determined from quark counting

$$
\begin{aligned}
\int_{0}^{1} x F_{3}\left(x, Q^{2}\right) d x & =3\left(1-\frac{\alpha_{s}\left(Q^{2}\right)}{\pi}\right) \\
& =3\left(1-\frac{A 1}{\log \left(Q^{2} / A_{0}^{2}\right)}-\frac{A 2}{\left[\log \left(Q^{2} / A_{0}^{2}\right)\right]^{2}}\right) \\
\int_{0}^{1} u_{v}\left(x, Q^{2}\right) d x & =2\left(1-\frac{A 1}{\log \left(Q^{2} / A_{0}^{2}\right)}-\frac{A 2}{\left[\log \left(Q^{2} / A_{0}^{2}\right)\right]^{2}}\right) \\
\int_{0}^{1} d_{v}\left(x, Q^{2}\right) d x & =\left(1-\frac{A 1}{\log \left(Q^{2} / A_{0}^{2}\right)}-\frac{A 2}{\left[\log \left(Q^{2} / A_{0}^{2}\right)\right]^{2}}\right)
\end{aligned}
$$

In addition a charge constraint is required

$$
\begin{aligned}
\frac{2}{3} \int_{0}^{1} u_{v}\left(x, Q^{2}\right) d x & -\frac{1}{3} \int_{0}^{1} d_{v}\left(x, Q^{2}\right) d x \\
& =\left(1-\frac{A 1}{\log \left(Q^{2} / A_{0}^{2}\right)}-\frac{A 2}{\left[\log \left(Q^{2} / A_{0}^{2}\right)\right]^{2}}\right),
\end{aligned}
$$

where the fit parameters $A 1$ and $A 2$ describe the normalization of the leading order and next-to-leading order terms respectively.

The light quark sea distributions are given by

$$
\begin{aligned}
x \bar{u}(x) & =x \bar{d}(x)=\frac{1}{2(\kappa+2)} x S(x) \\
& =\frac{1}{2(\kappa+2)}\left(A S(1-x)^{E S}+A S_{2}(1-x)^{E S_{2}}\right),
\end{aligned}
$$

where $S(x)$ is the light-quark sea density. $A S, A S_{2}, E S$, and $E S_{2}$ are defined in terms of fit parameters below, and the parameter $\kappa$ is the relative normalization to the strange sea, also defined below. The light quark sea distributions can be determined from the first two moments, because they decrease rapidly with $\mathrm{x}$

$$
\begin{aligned}
& S_{2}=\int_{0}^{1} x S d x=\frac{3}{4} D_{22}+\frac{1}{4} D_{12}, \\
& S_{3}=\int_{0}^{1} x^{2} S d x=\frac{3}{4} D_{23}+\frac{1}{4} D_{13},
\end{aligned}
$$

where the values for $D_{i j}$, which are completely specified by LO QCD, are given in [9]. $A S_{2}$ and $E S_{2}$ are evolved with $Q^{2}$ in the following way

$$
\begin{aligned}
& A S_{2}=A S_{20}+A S_{21} \ln \left(Q^{2}\right), \\
& E S_{2}=E S_{20}+E S_{21} \ln \left(Q^{2}\right) .
\end{aligned}
$$

$S_{2}, S_{3}, A S_{20}, A S_{21}, E S_{20}$, and $E S_{21}$ are parameters in the fit. $A S$ and $E S$ are constrained to match the moments $S_{2}$ and $S_{3}$.

$$
\begin{aligned}
E S & =\frac{S_{2}-A S_{2} /\left(E S_{2}+1\right)}{S_{3}-A S 2 /\left(\left(E S_{2}+1\right)\left(E S_{2}+2\right)\right)}-2, \\
A S & =(E S+1) \frac{S_{2}-A S_{2}}{E S_{2}+1} .
\end{aligned}
$$

The strange sea distribution can be measured from the dimuon inclusive cross section. The parameterization of the strange sea is a LO fit to the CCFR dimuon differential cross section [25],

$$
x s(x)=x \bar{s}(x)=\frac{\kappa}{2(\kappa+2)} x S S(x)
$$


where $S S(x)$ is the strange sea density. The relative normalization to the light quark seas is determined by the parameter $\kappa$,

$$
x s(x) \propto \kappa \frac{x \bar{u}_{R}(x)+x \bar{d}_{R}(x)}{2}(1-x)^{\alpha},
$$

where $\alpha$ describes the shape of the strange sea. Here the light quark sea densities $\bar{u}_{R}(x)$ and $\bar{d}_{R}(x)$, and the values of $\alpha=2.5$ and $\kappa=0.373$ are obtained from reference [25].

Momentum sum rule is constrained by the second moment of the gluon distribution $G_{2}=\int_{0}^{1} x g\left(x, Q^{2}\right) d x$

$$
\begin{aligned}
G_{2} & +\int_{0}^{1} \frac{1+R\left(x, Q^{2}\right)}{1+4 M^{2} x^{2} / Q^{2}}\left[x u_{v}\left(x, Q^{2}\right)\right. \\
& \left.+x d_{v}\left(x, Q^{2}\right)+x S\left(x, Q^{2}\right)\right] d x=1
\end{aligned}
$$

Additional small corrections are used to modify $u$ and $d$ distributions to account for the $u, d$ asymmetry observed in muon DIS and Drell-Yan data. Drell-Yan data from E866 [27] is used to constrain the $\bar{u}, \bar{d}$ asymmetry by the factor

$$
f(\bar{d} / \bar{u})=\frac{1}{\max \left(1-x\left(2.7-0.14 \ln \left(Q^{2}\right)-1.9 x\right), 0.1\right)}
$$

The modified light sea distributions $\overline{u^{\prime}}, \overline{d^{\prime}}$ are constrained by $\overline{u^{\prime}}+\overline{d^{\prime}}=\bar{u}+\bar{d}$ and have the form

$$
\begin{aligned}
& \overline{u^{\prime}}=\bar{u}\left(\frac{\bar{u}+\bar{d}}{\bar{u}+\bar{d} \cdot f(\bar{d} / \bar{u})}\right), \\
& \overline{d^{\prime}}=\bar{d}\left(\frac{\bar{u}+\bar{d}}{\bar{u}+\bar{d} \cdot f(\bar{d} / \bar{u})}\right) \cdot f(\bar{d} / \bar{u}) .
\end{aligned}
$$

The valence distributions are also modified for the $u_{v}, d_{v}$ asymmetry. The modified valence distributions $u_{v}^{\prime}, d_{v}^{\prime}$ are constrained by $u_{v}^{\prime}+d_{v}^{\prime}=u_{v}+d_{v}$ and have the form [3]

$$
\begin{aligned}
u_{v}^{\prime} & =\frac{u_{v}}{1+\delta(d / u) \cdot u_{v} /\left(u_{v}+d_{v}\right)}, \\
d_{v}^{\prime} & =\frac{d_{v}+u_{v} \cdot \delta(d / u)}{1+\delta(d / u) \cdot u_{v} /\left(u_{v}+d_{v}\right)}, \\
\delta(d / u) & =0.12079-1.3303 x+4.9829 x^{2} \\
& -8.4465 x^{3}+5.7324 x^{4} .
\end{aligned}
$$

The model fit parameters are obtained after an iterative loop in which the $n^{\text {th }}$ loop flux and cross section are re-extracted with the $(n-1)^{t h}$ loop model fit parameters. The $n^{\text {th }}$ loop model then determines new acceptance and smearing corrections which are used for the $(n+1)^{t h}$ cross section and fit. New radiative corrections are computed to correspond to each new model. The initial flux and cross section are determined using a starting model with the Buras-Gaemers PDF parameters from the best fit to CCFR data [3]. The process is iterated until the average relative change in the cross section data points compared to the previous iteration is less than $0.1 \%$ (within 3 iterations).

To constrain the high- $x$ and low $Q^{2}$ part of the model, which is important in order to model our flux data sample, we include data from charged-lepton scattering in the fit. The SLAC [28], BCDMS [29], and NMC [30] overlap with the kinematic range of our data set but extend to lower- $Q^{2}$. The charged-lepton data for $x$ in the range $0.4<x<0.7$, are included in the fit $\chi^{2}$ function along with the $\mathrm{NuTeV}$ data set to determine the best fit parameters. The charged-lepton data must first be corrected to $F_{2}^{\nu}$ using our model and corrected to an $A=56$ iron target using Equation 9. The normalization of the charged-lepton data relative to the $\mathrm{NuTeV}$ data is unconstrained in the fit.

Table I gives the $\mathrm{NuTeV}$ cross section model fit values for each parameter and their estimated uncertainties. A total of 19 parameters are fit in the model. Note that we do not expect that the parameterization extrapolated beyond the region of the $\mathrm{NuTeV}$ data set will be a good description of data there. 
TABLE I. The final fit parameters for the NuTeV cross section model.

\begin{tabular}{ccc}
\hline \hline Parameter & Value & Estimated Error \\
\hline$A_{0}$ & 0.583 & 0.017 \\
$A_{1}$ & 0.295 & 0.013 \\
$A_{2}$ & 0.17 & 0.03 \\
$E_{10}$ & 0.5333 & 0.0025 \\
$E_{11}$ & -0.028 & 0.011 \\
$E_{20}$ & 2.61 & 0.015 \\
$E_{21}$ & 1.31 & 0.045 \\
$A V_{2}$ & 637.0 & 75.0 \\
$E_{30}$ & 4.56 & 0.14 \\
$E_{40}$ & 12.5 & 0.35 \\
$S_{2}$ & 0.1625 & 0.0013 \\
$S_{3}$ & 0.0159 & 0.0004 \\
$G_{3}$ & 0.031 & 0.003 \\
$A S_{20}$ & 1.06 & 0.11 \\
$A S_{21}$ & 1.76 & 0.25 \\
$E S_{20}$ & 185.0 & 20.0 \\
$E S_{21}$ & 8.4 & 8.0 \\
$A_{H T}$ & 1.187 & 0.035 \\
$B_{H T}$ & 0.33 & 0.02 \\
\hline \hline
\end{tabular}

